\title{
Possible Avenues in Supersymmetry and Naturalness
}

by

\section{Hugues Beauchesne}

\author{
A thesis submitted to the \\ Faculty of Graduate and Postdoctoral Affairs \\ in partial fulfillment of the requirements \\ for the degree of
}

Doctor of Philosphy in Physics

\author{
Department of Physics \\ Carleton University \\ Ottawa-Carleton Institute of Physics \\ Ottawa, Canada
}

July 14, 2016

Copyright (c) 2016 Hugues Beauchesne 


\section{Abstract}

The purpose of this thesis is to investigate a set of possible realizations of Supersymmetry and naturalness in beyond the Standard Model physics. We will mostly be interested in models that combine both aspects, but this will not prevent us from investigating models that are only supersymmetric without being natural or that are natural without being supersymmetric. More precisely, we will look at four different cases.

First, we introduce our own variation of the Twin Higgs model, which we refer to as the spontaneous $\mathbb{Z}_{2}$ breaking Twin Higgs. The main feature of the model is that the $\mathbb{Z}_{2}$ breaking is spontaneous instead of explicit. In addition to the theoretical appeal of this, other advantages are that it reduces tuning and makes many mirror particles very heavy.

Second, we study collider constraints on Mini-Split models of Supersymmetry. More precisely, we study the current constraints on anomaly and gauge mediation, as well as their prospects for Run-II of the LHC and a future $100 \mathrm{TeV}$ proton collider. We assume the Higgsinos to be heavy and a slightly lighter third generation of squarks.

Third, we study constraints coming from electroweak precision tests on models of $U(1)_{R}$ Supersymmetry where the $R$-charge is associated with the lepton number. Contributions from new physics are encoded in a set of operators which are used to calculate corrections to a set of electroweak observables. The model is then constrained by fitting with experimental inputs.

Finally, we investigate whether supersymmetric models where the $U(1)_{R}$ symmetry is instead associated with the baryon number can lead to successful baryogenesis once this symmetry is broken. Indeed, the $U(1)_{R}$ symmetry is expected to be broken by either anomaly mediation or Planck scale suppressed operators, thereby 
breaking baryon number conservation. We find that it is possible to obtain the correct baryon density, but that a Mini-Split spectrum is required and that the $U(1)_{R}$ symmetry must be badly broken. We also find that the model does not require the Higgsinos to be as heavy as in the MSSM. 


\section{Acknowledgments}

A thesis is an endeavour in which someone seldom embarks himself alone. As such, I would like to acknowledge the people who would have helped me along the way.

First and foremost, I would like to thank my supervisor Dr Thomas Grégoire

for his continual support. His deep and clear understanding of particle physics have shaped me as a scientist.

I am indebted to Kevin Earl for his collaboration in several project. His thoroughness has insured the quality of our work.

I am also greatly indebted to the other faculties of the theory group: Dr Bruce Campbell, Dr Stephen Godfrey, Dr Pat Kalyniak, Dr Heather Logan and Dr Daniel Stolarski. The knowledge they have shared with me was invaluable. I would also like to thank the administrative staff of the physics department, Eva Lacelle and Joanne Martin, for their precious help.

I also acknowledge support from the Natural Science and Engineering Research Council of Canada as well as the government of Ontario.

Finally, I would like to thank my parents, Luc and Manon, as well as my sister Eve. I wish I would have had time to visit more often. 


\section{Stateman of Originality}

Chapters 2, 3 and 6 present review material of the Standard Model, Supersymmetry and Dirac gauginos respectively. Section 4.1 presents a review of the Twin Higgs. Section 5.1.1 provides a review of how Mini-Split spectra can arise in different scenarios. All of these are referenced accordingly.

Chapters 4, 5, 7 and 8 represent the new research of this thesis. These chapters are based on the author's publications and a paper to be published as listed below.

- Chapter 4 is based on Ref. [1]. Except Section 4.1 which is mentioned above, all results were derived independently by each of the authors. The calculations of Section 4.1.3 were performed by collaborator Kevin Earl and verified independently by the author.

- Chapter 5 is based on Ref. [2]. Except Section 5.1.1 which is mentioned above, all results were derived independently by each of the authors. Collaborator Kevin Earl wrote a code to simulate the CMS search of Ref. [3]. He is also responsible for writing scripts for event generation and running several of them.

- Chapter 7 is based on Ref. [4]. All results were derived independently by each of the authors. Collaborator Thomas Grégoire wrote a code to calculate the oblique parameters contributions from the Higgses.

- Chapter 8 represents work to be published. All results were derived independently by the author, Thomas Grégoire and Kevin Earl.

Apart from the reservations mentioned above, all of the research contained in this

thesis represents original work (to my knowledge) and has not been submitted by anyone else for publication or as a final project. 


\section{Contents}

1 Introduction 1

2 The Standard Model $\quad 6$

2.1 Field content and Lagrangian of the Standard Model . . . . . . . . 8

2.2 Electroweak symmetry breaking . . . . . . . . . . . . . . . . 11

2.3 Additional properties of the Standard Model . . . . . . . . . . . 15

2.3.1 Vacuum angles . . . . . . . . . . . . . . . . . . 15

2.3.2 Anomaly cancellation . . . . . . . . . . . . . 18

2.3.3 Baryon and lepton numbers . . . . . . . . . . . . . . 20

2.4 Shortcomings of the Standard Model . . . . . . . . . . . . . . 20

3 Supersymmetry 23

3.1 The SUSY algebra . . . . . . . . . . . . . . . 26

3.2 Superfields . . . . . . . . . . . . . . . . . . . . . . . 27

3.2.1 Chiral Superfields . . . . . . . . . . . . . . . . . 28

3.2.2 Vector superfields . . . . . . . . . . . . . . . . . . . 30

3.3 Supersymmetric Lagrangians . . . . . . . . . . . . . . . . . . . . 33

3.3.1 Chiral superfield kinetic terms . . . . . . . . . . . 33

3.3.2 Vector superfield kinetic terms . . . . . . . . . . . . . 35 
3.3 .3 Superpotential . . . . . . . . . . . . . . . . . . 36

3.3 .4 Potential . . . . . . . . . . . . . . . 36

3.4 The MSSM . . . . . . . . . . . . . . . . . . 37

3.4.1 Electroweak symmetry breaking . . . . . . . . . . . . . 40

3.5 Mediation mechanisms . . . . . . . . . . . . . . . . . . . 42

3.5 .1 Gravity mediation . . . . . . . . . . . . . . . 43

3.5 .2 Gauge mediation . . . . . . . . . . . . . . . . . . 44

3.5 .3 Anomaly mediation . . . . . . . . . . . . . . . 46

4 The spontaneous $\mathbb{Z}_{2}$ breaking Twin Higgs 48

4.1 The model . . . . . . . . . . . . . . . . . . . . . . . . . . 51

4.1 .1 The original Twin Higgs . . . . . . . . . . . . . . . . 51

$4.1 .2 \quad$ Spontaneous $\mathbb{Z}_{2}$ breaking . . . . . . . . . . . . . . 55

4.1 .3 Radiative corrections . . . . . . . . . . . . . 67

4.2 Numerical analysis of the fine-tuning . . . . . . . . . . . . . . 69

4.3 Possible UV completion . . . . . . . . . . . . . . . . . 73

5 Constraining Mini-Split Supersymmetry $\quad 75$

5.1 Theory . . . . . . . . . . . . . . . . . . . . 77

5.1 .1 Mini-Split models . . . . . . . . . . . . . . . . . . . 77

5.1 .2 Gaugino mass spectrum _. . . . . . . . . . . . . 80

5.1 .3 Gaugino decays . . . . . . . . . . . . . . . 83

$5.1 .4 \quad$ Higgs mass $\ldots \ldots \ldots \ldots \ldots$

5.2 Methodology and results . . . . . . . . . . . . . 86

$5.2 .1 \quad$ Parameter space $\ldots \ldots \ldots \ldots$. . . . . . . . . . 86

5.2 .2 Current LHC constraints . . . . . . . . . . . . . . . . . 89 
$5.2 .3 \quad$ Prospects at LHC $14 \ldots \ldots \ldots \ldots$

5.2 .4 Prospects at a $100 \mathrm{TeV}$ collider . . . . . . . . . . 96

5.3 Additional comments . . . . . . . . . . . . . . . . . . . . 102

6 Models of $U(1)_{R}$ Supersymmetry 106

6.1 Dirac gauginos . . . . . . . . . . . . . . . . . . 107

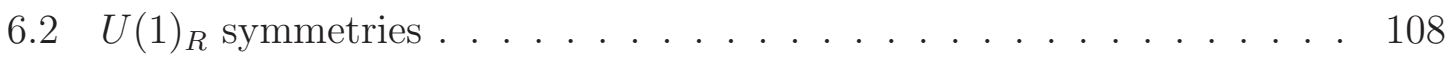

$6.2 .1 U(1)_{R_{1}}$ symmetry $\ldots \ldots \ldots \ldots \ldots \ldots$

$6.2 .2 U(1)_{R_{2}}$ symmetry $\ldots \ldots \ldots \ldots \ldots \ldots \ldots$

7 Electroweak precision measurements in supersymmetric models with

a $U(1)_{R}$ lepton number $\quad 112$

7.1 Necessary effective operators for EWPT . . . . . . . . . . 113

7.2 Coefficients of the EWPT operators . . . . . . . . . . 115

7.2 .1 Oblique parameters . . . . . . . . . . . . 116

7.2 .2 Four-fermion operators . . . . . . . . . . . . . . . 121

7.2 .3 Vertex modifying operators $\ldots \ldots \ldots \ldots$

7.2 .4 Loop contributions to $O_{\hat{W}} \ldots \ldots \ldots \ldots \ldots \ldots$

7.3 Method and results . . . . . . . . . . . . . 126

8 Baryogenesis in Supersymmetry with a broken $U(1)_{R}$ baryon number 131

$8.1 U(1)_{R}$ breaking $\ldots \ldots \ldots \ldots \ldots \ldots \ldots \ldots \ldots \ldots$

8.2 Baryon asymmetry . . . . . . . . . . . . . . . 137

8.2.1 Assumptions on the parameter space . . . . . . . . . 137

8.2 .2 Decay widths $\ldots \ldots \ldots \ldots \ldots$

8.2 .3 Net decay width to baryons . . . . . . . . . . . . . . 142

8.3 Annihilation and conversion cross sections . . . . . . . . . . . 144 
8.3.1 Interactions with Higgses . . . . . . . . . . . . . . . . . . 145

8.3.2 Interactions with quarks . . . . . . . . . . . . 146

8.4 Calculation of $\Omega_{\Delta B} \ldots \ldots \ldots \ldots$. . . . . . . . . . . . . . 147

8.5 Results and constraints . . . . . . . . . . . . . . . . . 149

8.6 Inclusion of light gluinos . . . . . . . . . . . . . . . . 152

$\begin{array}{lll}9 & \text { Summary } & 154\end{array}$

$\begin{array}{lr}\text { A Conventions } & 156\end{array}$

$\begin{array}{ll}\text { B Grassmann variables } & 159\end{array}$

C Van Der Waerden Notation 162 


\section{List of Tables}

2.1 Field content of the Standard Model. The index $m$ goes from 1 to 3, $a$ from 1 to 3 and $b$ from 1 to 8 . The $L$ and $R$ next to a spin $1 / 2$ field represents its chirality. . . . . . . . . . . . . . . . 9 9

3.1 Field content of the MSSM. The index $m$ goes from 1 to $3, a$ from 1 to 3 and $b$ from 1 to 8 . . . . . . . . . . . . . . . 38

5.1 Gluino pair production searches used to obtain current constraints. . 90

5.2 Signal regions for high MET. The background for $3 \mathrm{ab}^{-1}$ is also included.101

6.1 Superfields and their $U(1)$ charges. . . . . . . . . . . . . . . . . . . 108

6.2 Superfields and their $U(1)_{R}$ charges for three alternative models. . . . 109

7.1 Relevant observables. They are a modified list of those of Ref. [5]. . . 126

7.2 Masses for each plot in $\mathrm{GeV}$ and choice of lepton $a \cdot \chi^{2} /$ D.O.F. stands for the best fit of $\chi^{2}$ divided by the number of degrees of freedom. For the Standard Model, $\chi^{2} /$ D.O.F is 0.883. In all cases, $b_{T}=b_{S}=0 . . .127$ 


\section{List of Figures}

3.1 Contribution of the top and its partners to the Higgs mass. The quadratic divergences cancel. . . . . . . . . . . . . . . . .

4.1 Example of the different vevs as a function of $B_{\mu} / B_{\mu}^{\max }$. The parameters are $\mu_{1}=750 \mathrm{GeV}, \mu_{2}=850 \mathrm{GeV}, \alpha_{1}=-0.15, \alpha_{2}=0.2$ and

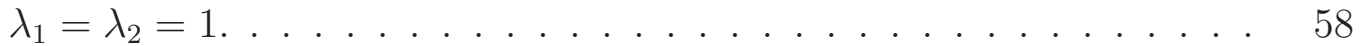

$4.2 F_{1}(\theta)$ and $F_{2}(\theta, \Omega)$ for different values of $\Omega \ldots \ldots \ldots \ldots$

4.3 $C\left(-\alpha_{2} / \alpha_{1}, \Omega\right)$ for various values for $-\alpha_{2} / \alpha_{1}$. Also shown is the corresponding value for Twin Higgs. . . . . . . . . . . . . . . . 62

4.4 Example of the different vevs as a function of $B_{\mu}$ for $\Omega>1$. The parameters are $\mu_{1}=1000 \mathrm{GeV}, \mu_{2}=850 \mathrm{GeV}, \alpha_{1}=-0.15, \alpha_{2}=0.15$ and $\lambda_{1}=\lambda_{2}=1 \ldots \ldots \ldots \ldots \ldots \ldots \ldots \ldots \ldots \ldots \ldots \ldots$

4.5 Example of $\Theta_{B}$ for the Twin Higgs and spontaneous $\mathbb{Z}_{2}$ breaking model with different values of $\mu_{2}$. The parameters for the Twin Higgs model are $\mu=750 \mathrm{GeV}, \alpha=-0.15$ and $\lambda=1$. The parameters for the spontaneous $\mathbb{Z}_{2}$ breaking model are $\mu_{1}=750 \mathrm{GeV}, \alpha_{1}=-0.15, \alpha_{2}=$ 0.2 and $\lambda_{1}=\lambda_{2}=1 \ldots \ldots \ldots \ldots \ldots \ldots$ 
4.6 Tuning of the spontaneous $\mathbb{Z}_{2}$ breaking Twin Higgs for a fixed $f_{1} / v$ of 3. The left panel shows the tuning in percentage and the right one the ratio of the tuning to the Twin Higgs benchmark value of $27.7 \%$. The gray area corresponds to the region where the constraints do not accept any solution. . . . . . . . . . . . . . . .

4.7 $f_{1} / v$ for the spontaneous $\mathbb{Z}_{2}$ breaking Twin Higgs for a fixed tuning of 20\%. The left panel shows $f_{1} / v$ and the right one the ratio of $f_{1} / v$ to the Twin Higgs benchmark value of 3.42. The gray area corresponds to the region where the constraints do not accept any solution. . . . .

5.1 Typical mass spectrum for (a) anomaly mediation and (b) gauge mediation. In (a), the masses appearing on the right side of Eq. (5.10) are taken to be $m_{\text {scalars }}=\mu=m_{3 / 2}=50 \mathrm{TeV}$ with $\tan \beta=2 . \operatorname{In}(\mathrm{b})$, the masses appearing on the right side of Eq. (5.13) are taken to be $m_{\text {scalars }}=\mu=\Lambda=200 \mathrm{TeV}$ with $\tan \beta=2 \ldots \ldots \ldots$

5.2 Branching ratios of the gluino for $M_{\tilde{G}}=1500 \mathrm{GeV}$ and $M_{\tilde{B}}=0 \mathrm{GeV}$. The third generation scalar masses are assumed to be degenerate and much heavier than the gauginos. . . . . . . . . . . . . . .

$5.395 \%$ CL exclusion limits for anomaly mediation for Run-I of the LHC. The yellow band corresponds to the $1 \sigma$ uncertainty on the gluino pair production cross section and the purple bands are the forbidden region of color breaking vacuum. Contour lines of constant $M_{\tilde{B}}, M_{\tilde{W}}, \mu$, and $\tan \beta$ are shown respectively in (a), (b), (c), and (d). . . . . . . 
5.4 95\% CL exclusion limits for gauge mediation for Run-I of the LHC. The yellow band corresponds to the $1 \sigma$ uncertainty on the gluino pair production cross section and the purple bands are the forbidden region of color breaking vacuum. The grey band corresponds to limits from direct electroweak searches. Contour lines of constant $M_{\tilde{B}}, M_{\tilde{W}}, \mu$, and $\tan \beta$ are shown respectively in (a), (b), (c), and (d) . . . . . .

5.5 95\% CL exclusion limits for anomaly mediation at LHC 14 for (solid) $300 \mathrm{fb}^{-1}$ and (dashed) $3000 \mathrm{fb}^{-1}$ integrated luminosity. The green band corresponds to the $1 \sigma$ uncertainty on the gluino pair production cross section for $300 \mathrm{fb}^{-1}$, the yellow band corresponds to the $1 \sigma$ uncertainty on the gluino pair production cross section for $3000 \mathrm{fb}^{-1}$, and the purple bands are the forbidden region of color breaking vacuum. Contour lines of constant $M_{\tilde{B}}, M_{\tilde{W}}, \mu$, and $\tan \beta$ are shown respectively in (a), (b),

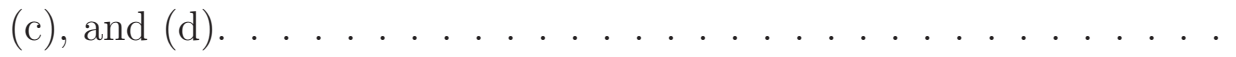

5.6 $5 \sigma$ discovery limits for anomaly mediation at LHC 14 for (solid) 300 $\mathrm{fb}^{-1}$ and (dashed) $3000 \mathrm{fb}^{-1}$ integrated luminosity. The green band corresponds to the $1 \sigma$ uncertainty on the gluino pair production cross section for $300 \mathrm{fb}^{-1}$, the yellow band corresponds to the $1 \sigma$ uncertainty on the gluino pair production cross section for $3000 \mathrm{fb}^{-1}$, and the purple bands are the forbidden region of color breaking vacuum. Contour lines of constant $M_{\tilde{B}}, M_{\tilde{W}}, \mu$, and $\tan \beta$ are shown respectively in (a), (b),

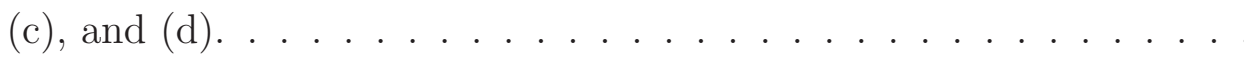


5.7 95\% CL exclusion limits for gauge mediation at LHC 14 for (solid) 300 $\mathrm{fb}^{-1}$ and (dashed) $3000 \mathrm{fb}^{-1}$ integrated luminosity. The green band corresponds to the $1 \sigma$ uncertainty on the gluino pair production cross section for $300 \mathrm{fb}^{-1}$, the yellow band corresponds to the $1 \sigma$ uncertainty on the gluino pair production cross section for $3000 \mathrm{fb}^{-1}$, and the purple bands are the forbidden region of color breaking vacuum. Contour lines of constant $M_{\tilde{B}}, M_{\tilde{W}}, \mu$, and $\tan \beta$ are shown respectively in (a), (b),

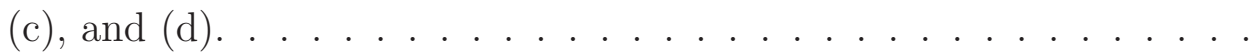

$5.85 \sigma$ discovery limits for gauge mediation at LHC 14 for (solid) 300 $\mathrm{fb}^{-1}$ and (dashed) $3000 \mathrm{fb}^{-1}$ integrated luminosity. The green band corresponds to the $1 \sigma$ uncertainty on the gluino pair production cross section for $300 \mathrm{fb}^{-1}$, the yellow band corresponds to the $1 \sigma$ uncertainty on the gluino pair production cross section for $3000 \mathrm{fb}^{-1}$, and the purple bands are the forbidden region of color breaking vacuum. Contour lines of constant $M_{\tilde{B}}, M_{\tilde{W}}, \mu$, and $\tan \beta$ are shown respectively in (a), (b), (c), and (d) . . . . . . . . . . . . . . . . . 100

5.9 95\% CL (dashed) exclusion and $5 \sigma$ (solid) discovery limits for anomaly mediation at a $100 \mathrm{TeV} p p$ collider with $3 \mathrm{ab}^{-1}$ integrated luminosity. The yellow band corresponds to the $1 \sigma$ uncertainty on the gluino pair production cross section for $95 \%$ exclusion, the green band corresponds to the $1 \sigma$ uncertainty on the gluino pair production cross section for $5 \sigma$ discovery, and the purple bands are the forbidden region of color breaking vacuum. Contour lines of constant $M_{\tilde{B}}, M_{\tilde{W}}, \mu$, and $\tan \beta$ are shown respectively in (a), (b), (c), and (d). . . . . . . . . 103 
$5.1095 \%$ CL (dashed) exclusion and $5 \sigma$ (solid) discovery limits for gauge mediation at a $100 \mathrm{TeV} p p$ collider with $3 \mathrm{ab}^{-1}$ integrated luminosity. The yellow band corresponds to the $1 \sigma$ uncertainty on the gluino pair production cross section for $95 \%$ exclusion, the green band corresponds to the $1 \sigma$ uncertainty on the gluino pair production cross section for $5 \sigma$ discovery, and the purple bands are the forbidden region of color breaking vacuum. Contour lines of constant $M_{\tilde{B}}, M_{\tilde{W}}, \mu$, and $\tan \beta$ are shown respectively in (a), (b), (c), and (d). . . . . . . . . . 104

7.1 Diagrams contributing to the oblique parameters [6]. Plain lines correspond to unspecified superpartners or leptons $a$. (a), (b), (c) and (d) correspond to $W, Y, \hat{S}$ and $\hat{T}$ respectively. $v$ stands for the insertion of a vev. . . . . . . . . . . . . . . . . . . 117

7.2 Diagram contributing to the four-fermion operators. Unidentified lines correspond to unspecified superpartners. . . . . . . . . . . . . . . 122

7.3 Corrections to gauge interactions arising from loops. Unidentified lines correspond to unspecified superpartners or leptons $a$. There are four possible types and similar diagrams exist for quarks. . . . . . . . . .

7.4 Region of allowed phase space for the mass combinations of Table 7.2 and for different values of $\mu$. The solid, dashed and dotdashed lines correspond respectively to 200,400 and $600 \mathrm{GeV}$. (a) is taken for $M_{1}^{D}=$ $M_{2}^{D}=700 \mathrm{GeV}$ and (b) for $M_{1}^{D}=M_{2}^{D}=1000 \mathrm{GeV}$. Both are taken at $v_{a}=25 \mathrm{GeV}$ and lepton $a$ is the electron. The contours correspond to $95.45 \%$ confidence level. . . . . . . . . . . . . . . . . . . . . . 128 
7.5 Region of allowed phase space for the mass combinations of Table 7.2 and for different choices of lepton $a$ The solid, dashed and dotdashed lines correspond respectively to the electron, muon and tau. Both figures have $\lambda_{S}=0$ and (a) has $M_{1}^{D}=M_{2}^{D}=700 \mathrm{GeV}$ and (b) has $M_{1}^{D}=M_{2}^{D}=1000 \mathrm{GeV}$. The contours correspond to $95.45 \%$ confidence level. . . . . . . . . . . . . . . . . . . . . . . 129

7.6 $\hat{T}$ and $\hat{S}$ parameters as a function of $\lambda_{T}$ for the masses of Table 7.2 and different values of $M_{2}^{D}$. The solid, dashed and dotdashed lines correspond respectively to 500, 700 and $1000 \mathrm{GeV}$. Both are taken at $v_{a}=25 \mathrm{GeV}, M_{1}^{D}=M_{2}^{D}$ and with $\lambda_{S}=0$. The horizontal line corresponds to what the limit on $\hat{T}$ would be at $95.45 \%$ if only its corresponding operator would be present and $Y=0$. Its value is 1.04 $\times 10^{-3} \ldots \ldots \ldots \ldots \ldots \ldots$

8.1 Tree-level baryon number breaking decay of binos. . . . . . . . . . . . 138

8.2 Baryon conserving decays of $\chi_{2}^{B}$ to $\chi_{1}^{B}$ and quarks. The diagram with

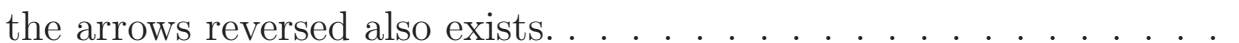

8.3 Baryon conserving decays of $\chi_{2}^{B}$ to $\chi_{1}^{B}$ and Higgses. Other diagrams exist. . . . . . . . . . . . . . . . . . 140

8.4 Decay of $\chi_{2}^{B}$ to $\chi_{1}^{B}$ and a photon. The photon can also be inserted on the squark line and the arrow flow can be reversed in both cases. . . . 143

8.5 Loop decay of $\chi_{2}^{B}$ via virtual $\chi_{1}^{B} \ldots \ldots \ldots$. . . . . . . . . . 144

8.6 (a) Annihilation to Higgses. There also exist other diagrams. (b) Example of bino conversion via Higgs scattering. There also exist other

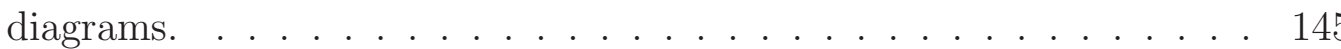


8.7 (a) Example of $s$-channel annihilation of a single bino. (b) Example of t-channel annihilation of a single bino. . . . . . . . . . . . 146

8.8 (a) Example of bino pair annihilation to $d_{2} \bar{d}_{2}$. There is also a diagram with the incoming lines crossed. (b) Example of bino conversion via scattering off quarks. There is also an $s$-channel diagram. . . . . . . . 147

8.9 Contour plots of constant $\Omega_{\Delta B}$. The blue region corresponds to $\chi_{2}^{B}$ decaying before electroweak phase transition. The yellow region corresponds to $\chi_{2}^{B}$ decaying before freeze-out. The pink region is excluded by washout. . . . . . . . . . . . . . . . . . . 150

8.10 Contour plots of constant $\Omega_{\Delta B}$. The blue region corresponds to $\chi_{2}^{B}$ decaying before electroweak phase transition. The yellow region corresponds to $\chi_{2}^{B}$ decaying before freeze-out. . . . . . . . . . . . . 151 


\section{Chapter 1}

\section{Introduction}

The Standard Model (SM) of particle physics is an extremely successful theory which synthesizes our current understanding of high energy physics. It is based on a specific symmetry structure and all known elementary particles, be they leptons, quarks or gauge bosons, can be embedded in representations of the corresponding symmetry groups. Even more, all interactions, with the notable exception of gravity, have been verified to be in good agreement with the predictions of the Standard Model.

However, the Standard Model is not without its flaws. From an experimental point of view, the most tangible problem is that neutrinos are now known to be massive, which goes against the predictions of the Standard Model. This can however be easily accommodated by simple extensions. On a slightly more indirect level, cosmological evidences now show that the Universe contains about $25 \%$ of dark matter, i.e. matter that is not seen but is necessary to account for many cosmological phenomena. The required properties of such particles are incompatible with the different particles of the Standard Model and the latter must therefore be extended. The observed asymmetry between the matter and antimatter content of the Universe is also difficult to explain with the Standard Model alone. 
The Standard Model also possesses many shortcoming from a theoretical point of view. The strong $C P$ problem, which stems from the QCD vacuum angle being unnaturally small, is a famous example of the Standard Model needing to be extremely fine-tuned. The presence of three generations of fermions or even the hierarchy between their masses is at best puzzling.

Perhaps more importantly and one of the main themes of this thesis, the Standard Model suffers from the hierarchy problem. The potential of the Higgs field is unstable under radiative corrections which results in a Higgs boson whose mass should naturally be of order of the Planck scale. Since the Higgs mass is now measured to be around the electroweak scale, a fine-tuning of order one part in $10^{32}$ is required. It is certainly hard to believe that such a coincidence is accidental and as such the Standard Model is said to be unnatural.

Many theories have tried to address the hierarchy problem and are said to attempt to restore naturalness. The most famous of them is certainly Supersymmetry (SUSY). This extension of the Standard Model can be seen as an expansion of the symmetries of space-time and, in its simplest form, posits the existence of a partner of different spin for every known elementary particle. In addition to solving the hierarchy problem, Supersymmetry provides a dark matter candidate and leads to gauge coupling unification. A possible drawback of Supersymmetry is that it requires at least some of the partners to be around the electroweak scale if a small hierarchy problem is to be avoided. Since constraints on such particles are becoming very strong, Supersymmetry is starting to be under tension.

Taking these considerations into account, the concept of neutral naturalness has been proposed. The driving force behind this idea is that the constraints on most superpartners are so strong because they can easily be created at hadron colliders. If 
instead the stabilizing partners interacted only weakly with the Standard Model, it would be difficult to produce them and as such their constraints would be far weaker. This is the concept behind neutral naturalness, i.e. having the Higgs mass stabilized by partners that are not charged under the Standard Model gauge groups.

In this context, the purpose of the present thesis is to study possible future avenues for both Supersymmetry and naturalness. As Supersymmetry tries to address naturalness, we will more often than not cover both subjects at the same time. However, we will also look at some supersymmetric models that are not natural but still present some advantages and we will study as well an example of naturalness that does not assume Supersymmetry. More precisely, we will look at four different models.

One of the foremost examples of neutral naturalness is the Twin Higgs. This model rests on a global $S U(4)$ symmetry that is broken spontaneously to $S U(3)$, with the Higgs being identified with one of the resulting pseudo-Goldstone bosons. The model contains a Standard Model sector and a mirror sector, with a $\mathbb{Z}_{2}$ symmetry between the two protecting the Higgs mass. This $\mathbb{Z}_{2}$ symmetry must however be softly broken if the model is to be phenomenologically viable and this breaking must be tuned against the $S U(4)$ breaking, leading to a small amount of fine-tuning. We propose a new model where the $\mathbb{Z}_{2}$ symmetry is instead broken spontaneously. In addition to being more attractive theoretically, this model has the advantages of reducing the necessary tuning and making many mirror particles very heavy.

Putting naturalness considerations aside for a moment, Supersymmetry still presents many advantages if one is willing to accept a certain amount of fine-tuning. This is the driving idea behind Split-SUSY. In this class of models, the scalar partners are assumed very heavy, which relaxes several constraints, and only fermion partners 
are close to the electroweak scale. This can still lead to gauge coupling unification and can still provide a dark matter candidate. However, the recent measurements of the Higgs mass impose an upper limit on scalar masses. This led to the introduction of Mini-Split models, where the splitting between fermion and boson superpartner masses is only a few orders of magnitude. We study the current collider constraints on such models, more specifically in the case of anomaly and gauge mediation. Prospects for Run-II of the LHC and a future $100 \mathrm{TeV}$ proton collider are also included.

Returning to naturalness, constraints on many superpartners can be considerably reduced if the partners of gauge bosons are Dirac instead of Majorana, as is usually the case. One of the main advantages of this is that it allows the imposition of a $U(1)_{R}$ symmetry. There is however a certain freedom on the exact realization of this symmetry. In one realization, the $U(1)_{R}$ can be associated with the lepton number. In this case, it is possible for the partner of one of the neutrinos to acquire a vacuum expectation value (vev) and play the role of a Higgs. However, such a model is constrained by electroweak precision tests (EWPT). We study such constraints by coding the effects of the model in terms of effective operators, calculating the contributions to a set of electroweak observables and performing a global fit.

Finally, this $U(1)_{R}$ symmetry can also be associated with the baryon number. The symmetry is however expected to be broken by anomaly mediation or by Planck scale suppressed operators. This scenario can then possibly be used to explain the baryon asymmetry observed in the Universe, i.e. baryogenesis. More precisely, we study the requirements for correct baryogenesis in Supersymmetry with a broken $U(1)_{R}$ baryon number. We find that similarly to the MSSM a Mini-Split spectrum is required, but that the extended Higgs sector allows for lighter Higgsinos. Unfortunately, obtaining the correct baryon density requires the $U(1)_{R}$ symmetry to be badly 
broken.

This thesis is organized as follows. A short review of the Standard Model is first given followed by an introduction to Supersymmetry. Our Twin Higgs model is then presented. The following chapter contains our constraints on Mini-Split models. Models of $U(1)_{R}$ Supersymmetry are then summarized. EWPT constraints on the $U(1)_{R}$ lepton number model follow. Finally, baryogenesis from Supersymmetry with a broken $U(1)_{R}$ baryon number is discussed. Three appendices respectively summarize the conventions used in this thesis, Grassmann variables and the van der Waerden notation. 


\section{Chapter 2}

\section{The Standard Model}

Experiments in particle physics have led to the discovery of several particles which are believed to be fundamental. Though some of their properties vary wildly, they still fall in distinct categories.

Gauge bosons are spin 1 particles responsible for mediating the electromagnetic, weak and strong interactions. Gluons are a set of eight electrically neutral massless gauge bosons which are responsible for the strong force. The $W$ and $Z$ bosons mediate the electroweak force and are amongst the heaviest known particles. The $W$ boson comes in two varieties of charge $\pm e$, while the $Z$ boson is electrically neutral. The photon is massless, neutral and mediates the electromagnetic force. All neutral gauge bosons are their own antiparticle, while the two $W$ 's are antiparticles of each other.

Quarks are massive spin 1/2 fermions whose defining property is that they interact strongly. As a consequence of this, they are subject to confinement, i.e. that a free quark cannot exist by itself. In conjunction with gluons, they are the building blocks of mesons and baryons. Quarks come with two possible electric charges, $+(2 / 3) e$ and $-(1 / 3) e$, and to each charge is associated three different flavours. The three types of charge $+(2 / 3) e$ quarks are up, charm and top, while those of charge 
-(1/3)e are down, strange and bottom. For each quark, there exists an antiquark of equal in magnitude but opposite charge.

The leptons on the other hand are spin $1 / 2$ fermions whose defining property is that they do not interact strongly. Leptons come either with charge $-e$ (the charged leptons) or are neutral (the neutrinos). The three types of charged leptons are the electron, muon and tau. Each of them possesses an antiparticle of charge $+e$. The neutrinos are usually referred to as electron, muon and tau neutrinos. It is not known at this point whether the neutrinos are their own antiparticle or not. Charged leptons have well measured masses, while those of neutrinos are not yet determined but are known to be non-zero.

Finally, the last known fundamental particle is the recently discovered Higgs boson $[7,8]$. It is a neutral spin 0 boson that is both massive and its own antiparticle.

The Standard Model (SM) of particle physics is the theory that codifies all of this knowledge in a mathematically self-consistent framework. It is the theory that explains (almost) all of our current understanding of particle physics and is one of the most accurately tested theories in all of science. It consists of a quantum field theory based on the symmetry group $S U(3)_{c} \times S U(2)_{L} \times U(1)_{Y}$. The electroweak group $S U(2)_{L} \times U(1)_{Y}$ is broken spontaneously to its diagonal subgroup $U(1)_{\mathrm{EM}}$ by the Higgs mechanism. This in turn provides a mass to the electroweak gauge bosons, charged leptons and quarks. The goal of this chapter is to summarize briefly the most relevant or salient features of the Standard Model.

This chapter is organized as follows. The field content and Lagrangian of the Standard Model are first presented. The process of electroweak symmetry breaking and its consequences are then explained. A few properties of the model follow. Finally, a few shortcomings of the Standard Model are mentioned. 
This chapter draws inspiration from Refs. [9, 10] without explicitly following any of them. We refer to these sources for more details.

\subsection{Field content and Lagrangian of the Standard Model}

The field content of the Standard Model is summarized in Table 2.1. The convention used in this chapter for spin 1/2 fields is that they are 4-component spinors upon which acts either a left-handed or right-handed projector depending on their chirality. For example, $\psi_{L}=P_{L} \psi$, where $\psi$ is a 4-component spinor whose right-handed part is irrelevant. The $S U(2)_{L}$ components of $L_{L}^{m}$ and $Q_{L}^{m}$ are given special names as $L_{L}^{m}=\left(\nu_{L}^{m}, \mathcal{E}_{L}^{m}\right)$ and $Q_{L}^{m}=\left(\mathcal{U}_{L}^{m}, \mathcal{D}_{L}^{m}\right)$. The field $Q_{L}^{m}$ contains the left-handed parts of the up and down quarks and their right-handed parts are $U_{R}^{m}$ and $D_{R}^{m}$ respectively. The field $L_{L}^{m}$ contains the left-handed parts of the neutrinos and charged leptons, while only the charged leptons have a right-handed part labeled $E_{R}^{m}$. The gauge bosons of $U(1)_{Y}, S U(2)_{L}$ and $S U(3)_{c}$ are respectively $B_{\mu}, W_{\mu}^{a}$ and $G_{\mu}^{b}$. The field $H$ is the Higgs doublet. The index $m$ on all fermions corresponds to the generation and runs from 1 to 3 . The $S U(3)_{c}$ and $S U(2)_{L}$ indices are not written but assumed implicitly.

The Lagrangian of the Standard Model is the most general Lagrangian that can be written in terms of these fields and that is both renormalizable and invariant under the $S U(3)_{c} \times S U(2)_{L} \times U(1)_{Y}$ gauge group. More explicitly, a generic infinitesimal gauge transformation can be parametrized by three parameters associated with $S U(3)_{c}, S U(2)_{L}$ and $U(1)_{Y}$, which we call $\alpha^{a}, \beta^{a}$ and $\epsilon$ respectively. We label a generic fermion or the Higgs doublet by $\psi_{i}$. We define $T_{3}^{a}$ and $T_{2}^{a}$ as the generator of $S U(3)_{c}$ and $S U(2)_{L}$ under which this field transforms and label its $U(1)_{Y}$ charge 


\begin{tabular}{|c|c|c|c|c|}
\hline & $S U(3)_{C}$ & $S U(2)_{L}$ & $U(1)_{Y}$ & Spin \\
\hline$H$ & $\mathbf{1}$ & $\mathbf{2}$ & $1 / 2$ & 0 \\
\hline$Q_{L}^{m}$ & $\mathbf{3}$ & $\mathbf{2}$ & $1 / 6$ & $1 / 2$ \\
\hline$U_{R}^{m}$ & $\mathbf{3}$ & $\mathbf{1}$ & $2 / 3$ & $1 / 2$ \\
\hline$D_{R}^{m}$ & $\mathbf{3}$ & $\mathbf{1}$ & $-1 / 3$ & $1 / 2$ \\
\hline$L_{L}^{m}$ & $\mathbf{1}$ & $\mathbf{2}$ & $-1 / 2$ & $1 / 2$ \\
\hline$E_{R}^{m}$ & $\mathbf{1}$ & $\mathbf{1}$ & -1 & $1 / 2$ \\
\hline$B_{\mu}$ & $\mathbf{1}$ & $\mathbf{1}$ & 0 & 1 \\
\hline$W_{\mu}^{a}$ & $\mathbf{1}$ & $\mathbf{3}$ & 0 & 1 \\
\hline$G_{\mu}^{b}$ & $\mathbf{8}$ & $\mathbf{1}$ & 0 & 1 \\
\hline
\end{tabular}

Table 2.1: Field content of the Standard Model. The index $m$ goes from 1 to 3, $a$ from 1 to 3 and $b$ from 1 to 8 . The $L$ and $R$ next to a spin $1 / 2$ field represents its chirality.

by $Y$. The latter is known as the weak hypercharge. Then, the Lagrangian of the Standard Model is invariant under the simultaneous transformation of all fields

$$
\begin{aligned}
\psi_{i} & \rightarrow \exp \left[-i\left(\alpha^{a} T_{3}^{a}+\beta^{a} T_{2}^{a}+\epsilon Y\right)\right] \psi_{i}, \\
G_{\mu}^{a} & \rightarrow G_{\mu}^{a}+\frac{1}{g_{s}} \partial_{\mu} \alpha^{a}+f_{c}^{a b c} G_{\mu}^{b} \alpha^{c}, \\
W_{\mu}^{a} & \rightarrow W_{\mu}^{a}+\frac{1}{g} \partial_{\mu} \beta^{a}+f_{L}^{a b c} W_{\mu}^{b} \beta^{c}, \\
B_{\mu} & \rightarrow B_{\mu}^{a}+\frac{1}{g^{\prime}} \partial_{\mu} \epsilon,
\end{aligned}
$$

where $g_{s}, g$ and $g^{\prime}$ are the coupling constants associated with $S U(3)_{c}, S U(2)_{L}$ and $U(1)_{Y}$ respectively, while $f_{c}^{a b c}$ and $f_{L}^{a b c}$ are the structure constants of $S U(3)_{c}$ and $S U(2)_{L}$ respectively.

The Lagrangian consists of multiple components which are given here. The part responsible for the kinematic terms of the gauge bosons and, in the non-abelian case, for their self-interactions is

$$
\mathcal{L}_{\text {gauge I }}=-\frac{1}{4} B_{\mu \nu} B^{\mu \nu}-\frac{1}{4} W_{\mu \nu}^{a} W^{a \mu \nu}-\frac{1}{4} G_{\mu \nu}^{a} G^{a \mu \nu} .
$$


Field strengths are defined in the general case via

$$
A_{\mu \nu}^{a}=\partial_{\mu} A_{\nu}^{a}-\partial_{\nu} A_{\mu}^{a}+g f^{a b c} A_{\mu}^{b} A_{\nu}^{c}
$$

where $A_{\mu}^{a}$ represents a generic set of gauge bosons, $g$ is the coupling constant of the corresponding group and $f^{a b c}$ its structure constants. In addition, the following pure gauge Lagrangian is also allowed

$$
\mathcal{L}_{\text {gauge II }}=\frac{g^{\prime 2} \Theta^{\prime}}{64 \pi^{2}} \epsilon^{\alpha \beta \mu \nu} B_{\alpha \beta} B_{\mu \nu}+\frac{g^{2} \Theta_{L}}{64 \pi^{2}} \epsilon^{\alpha \beta \mu \nu} W_{\alpha \beta}^{a} W_{\mu \nu}^{a}+\frac{g_{s}^{2} \Theta_{s}}{64 \pi^{2}} \epsilon^{\alpha \beta \mu \nu} G_{\alpha \beta}^{a} G_{\mu \nu}^{a}
$$

where $\epsilon^{\alpha \beta \mu \nu}$ is the completely antisymmetric Levi-Civita symbol with $\epsilon^{0123}=1$ and the different $\Theta$ 's represent the vacuum angles of the different gauge groups. The kinetic terms of the fermions, as well as their interactions with the gauge bosons, are encoded in the Lagrangian

$$
\mathcal{L}_{\text {matter }}=\sum_{\psi=Q, U, D, L, E} i \bar{\psi} \not D \psi
$$

The $\not D$ stands for $\gamma^{\mu} D_{\mu}$, where $\gamma^{\mu}$ are the Dirac matrices and $D_{\mu}$ is the covariant derivative. The latter is defined such that a field and its covariant derivative transform the same way under gauge transformation. For example, the covariant derivative of $Q_{L}^{m}$ is given by

$$
D_{\mu} Q_{L}^{m}=\left(\partial_{\mu}+i g_{s} G_{\mu}^{a} \frac{\lambda^{a}}{2}+i g W_{\mu}^{a} \frac{\sigma^{a}}{2}+i \frac{1}{6} g^{\prime} B_{\mu}\right) Q_{L}^{m}
$$

where $\lambda^{a}$ are the Gell-Mann matrices and $\sigma^{a}$ the Pauli matrices. The Higgs field $H$ 
has a kinetic Lagrangian of

$$
\mathcal{L}_{\text {Higgs kinetic }}=\left|D_{\mu} H\right|^{2},
$$

and a potential of

$$
V_{\mathrm{Higgs}}=-\mu^{2} H^{\dagger} H+\lambda\left(H^{\dagger} H\right)^{2} .
$$

Finally, the Higgs field links left-handed and right-handed fermions via Yukawa interactions

$$
\mathcal{L}_{\text {Yukawa }}=-y_{m n}^{e} \bar{L}_{L}^{m} H E_{R}^{n}-y_{m n}^{d} \bar{Q}_{L}^{m} H D_{R}^{n}-y_{m n}^{u} \bar{Q}_{L}^{m} \tilde{H} U_{R}^{n}+\text { h.c. },
$$

where $\tilde{H}=i \sigma_{2} H^{*}$. In summary, the Lagrangian of the Standard Model is

$$
\mathcal{L}_{\mathrm{SM}}=\mathcal{L}_{\text {gauge I }}+\mathcal{L}_{\text {gauge II }}+\mathcal{L}_{\text {matter }}+\mathcal{L}_{\text {Higgs kinetic }}+\mathcal{L}_{\text {Yukawa }}-V_{\text {Higgs }} .
$$

\section{$2.2 \quad$ Electroweak symmetry breaking}

Even though the Standard Model possesses many symmetries, not all of them are symmetries of the ground state. Indeed, the potential in Eq. (2.8) is invariant under gauge transformations of the Higgs field, but is minimized for a non-zero expectation value of this field

$$
\langle H\rangle=\left(\begin{array}{c}
0 \\
\frac{\mu}{\sqrt{2 \lambda}}
\end{array}\right) .
$$

The direction of the minimum is unphysical, as all directions are equivalent up to a gauge transformation. The important point is that, since the minimum is removed from the origin, the symmetry of the system is broken. This is the phenomenon 
known as spontaneous symmetry breaking. It is the origin of electroweak symmetry breaking in the Standard Model. The Higgs doublet can then be decomposed as

$$
H=\left(\begin{array}{c}
H^{+} \\
\frac{1}{\sqrt{2}}\left(v+h+i A^{0}\right)
\end{array}\right),
$$

where $h$ and $A^{0}$ are respectively $C P$-even and $C P$-odd real scalars, $H^{+}$is a charged complex scalar and $v=\mu / \sqrt{\lambda}$ is the vacuum expectation value (vev) of the Higgs field. The fields $H^{+}$and $A^{0}$ are Goldstone bosons and will become the longitudinal modes of massive gauge bosons associated with the broken symmetries. The field $h$ is the famous Higgs boson and is the only physical component of $H$.

The presence of a constant in the Higgs doublet spoils the invariance of the vacuum under most electroweak transformations. If they are to remain symmetries, transformations are required to leave the second component of the Higgs field untouched. As such, the only electroweak symmetry that is unbroken is the one which acts on the Higgs field with the generator $\operatorname{diag}(1,0)=\left(\sigma_{3}+\mathbb{I}_{2 \times 2}\right) / 2=T_{3}+Y$. This symmetry corresponds to performing a very specific combination of $S U(2)_{L}$ and $U(1)_{Y}$ transformations at the same time and is referred to as $U(1)_{\mathrm{EM}}$. As such, we say that $S U(2)_{L} \times U(1)_{Y}$ is broken spontaneously to its diagonal subgroup $U(1)_{\mathrm{EM}}$. The $U(1)_{\mathrm{EM}}$ charge of a field is therefore given by

$$
Q=T_{3}+Y
$$

where in this case $T_{3}$ refers to the eigenvalue of the third generator of $S U(2)_{L}$ associated with this field. For example, $T_{3}=1 / 2$ for $\mathcal{E}_{L}$ and $T_{3}=-1 / 2$ for $\nu_{L}$. Since $S U(3)_{c}$ acts trivially on the Higgs doublet, this gauge group is unbroken. 
Substituting Eq. (2.12) into Eq. (2.7) leads to masses for the gauge bosons. The resulting mass eigenstates are

$$
\begin{aligned}
W_{\mu}^{ \pm} & =\frac{1}{\sqrt{2}}\left(W_{\mu}^{1} \mp i W_{\mu}^{2}\right), \\
Z_{\mu} & =-\sin \theta_{W} B_{\mu}+\cos \theta_{W} W_{\mu}^{3}, \\
A_{\mu} & =\cos \theta_{W} B_{\mu}+\sin \theta_{W} W_{\mu}^{3},
\end{aligned}
$$

where the Weinberg angle $\theta_{W}$ is given via

$$
\sin \theta_{W}=\frac{g^{\prime}}{\sqrt{g^{\prime 2}+g^{2}}}
$$

The field $A_{\mu}$ remains massless and is associated with the unbroken $U(1)_{\mathrm{EM}}$. The heavy gauge bosons acquire masses of

$$
M_{W}=\frac{g v}{2}, \quad M_{Z}=\frac{\sqrt{g^{\prime 2}+g^{2}}}{2} v .
$$

The gauge coupling constant associated with the unbroken $U(1)_{\mathrm{EM}}$ is

$$
e=\frac{g g^{\prime}}{\sqrt{g^{2}+g^{\prime 2}}}
$$

and corresponds to the absolute value of the charge of the electron.

Replacing the Higgs by its expectation value in the Yukawa interactions of Eq. (2.9) leads to Dirac masses for fermions. For up-type quarks, this gives

$$
\mathcal{L}_{u \text { masses }}=-\frac{y_{m n}^{u} v}{\sqrt{2}} \overline{\mathcal{U}}_{L}^{m} U_{R}^{n}+\text { h.c. }
$$

The Yukawa matrix can be diagonalized by passing to a basis $\mathcal{U}_{L}^{\prime a}$ and $U_{R}^{\prime a}$ defined 
via

$$
\mathcal{U}_{L}^{a}=U_{(u)}^{a b} \mathcal{U}_{L}^{\prime b}, \quad U_{R}^{a}=V_{(u)}^{a b} U_{R}^{\prime b}
$$

Defining

$$
u^{a}=\mathcal{U}_{L}^{\prime a}+U_{R}^{\prime a}
$$

and using the fact that $\bar{u} u=\overline{\mathcal{U}}_{L}^{\prime} U^{\prime}{ }_{R}+\bar{U}_{R}^{\prime} \mathcal{U}_{L}^{\prime}$, Eq. (2.18) can be rewritten as

$$
\mathcal{L}_{u \text { masses }}=-\frac{v}{\sqrt{2}}\left(U_{(u)}^{i m \dagger} y_{m n}^{u} V_{(u)}^{n i}\right) \bar{u}^{i} u^{i}=-\frac{y_{m m}^{\prime u} v}{\sqrt{2}} \bar{u}^{m} u^{m}
$$

where the mass matrix $y^{\prime \prime}$ is now diagonal. The same can be done for down-type quarks. However, rotation matrices will not in general be the same for the two $S U(2)_{L}$ components of $Q_{L}$. As such, interactions mediated by $W$ bosons, which interchange up and down-type quarks, will not be diagonal in flavour space. This is represented by the Lagrangian

$$
\mathcal{L}_{\mathrm{cc}}=i \frac{g}{\sqrt{2}} U_{(u)}^{\dagger}{ }_{(d)}^{i n} W_{\mu}^{+} \bar{u}^{m} \gamma^{\mu} P_{L} d^{n}+\text { h.c. }=i \frac{g}{\sqrt{2}} V_{m n} W_{\mu}^{+} \bar{u}^{m} \gamma^{\mu} P_{L} d^{n}+\text { h.c. }
$$

where $V_{m n}$ is the Cabibbo-Kobayashi-Maskawa (CKM) matrix. It is in general a complex matrix. However, field redefinitions can be used to rotate away all phases except one. The remaining phase breaks invariance under charge and parity inversion, which is commonly known as $C P$-invariance.

The same procedure can be applied to charged leptons with a few differences. Since there is no equivalent Yukawa term for neutrinos, these can be rotated among each other freely. It is then possible to rotate neutrinos the same way as left-handed charged leptons. Interactions between the $W$ bosons and leptons can therefore be made diagonal in flavour space. Should the neutrinos be massive, we would not in 
general be able to rotate them freely. This would lead to the equivalent of the CKM matrix for leptons, known as the Pontecorvo-Maki-Nakagawa-Sakata (PMNS) matrix.

\subsection{Additional properties of the Standard Model}

In addition to the content of the past two sections, the Standard Model possesses many interesting properties. In this section, we mention some that will play an important role in later chapters or that are simply worth noting.

\subsubsection{Vacuum angles}

Not all vacuum angles appearing in Eq. (2.4) have physical consequences. First, the $S U(2)_{L}$ vacuum angle can be rotated away by field redefinitions and by exploiting the chiral anomaly.

In the context of quantum field theory, an anomaly is when a symmetry of a classical theory fails to remain a symmetry of its quantum counterpart. This leads to quantities, which would be conserved classically under Noether's theorem, to not be preserved anymore. This is not necessarily detrimental, as in the present example.

In the absence of Yukawa interactions, the Standard Model Lagrangian would be invariant under the multiplication of an individual spinor $\psi_{i}$ by a phase, i.e. $\psi_{i} \rightarrow \exp (i \alpha) \psi_{i}$ where $\alpha$ (which we assume constant) parametrizes the transformation. ${ }^{1}$ It can be generalized to multiplying multiplets of Weyl spinors by $\exp \left(i \alpha T_{a}\right)$, where $T_{a}$ is a generator of the gauge group under which the field transforms. Such transformations are referred to as chiral transformations. For convenience sake, we assume the Lagrangian has been rewritten in terms of left-handed Weyl spinors only.

\footnotetext{
${ }^{1}$ We assume all spinors in a multiplet are submitted to the same transformation.
} 
The generators are then given by

\begin{tabular}{c|ccccc} 
& $Q_{L}$ & $U_{L}$ & $D_{L}$ & $L_{L}$ & $E_{L}$ \\
\hline$Y$ & $\frac{1}{6} \mathbb{I}_{6 \times 6}$ & $-\frac{2}{3} \mathbb{I}_{3 \times 3}$ & $\frac{1}{3} \mathbb{I}_{3 \times 3}$ & $-\frac{1}{2} \mathbb{I}_{2 \times 2}$ & 1 \\
$S U(2)_{L}$ & $\mathbb{I}_{3 \times 3} \otimes \frac{\sigma^{a}}{2}$ & 0 & 0 & $\frac{\sigma^{a}}{2}$ & 0 \\
$S U(3)_{c}$ & $\frac{\lambda^{a}}{2} \otimes \mathbb{I}_{2 \times 2}$ & $-\frac{\lambda^{a *}}{2}$ & $-\frac{\lambda^{a *}}{2}$ & 0 & 0
\end{tabular}

where $\sigma^{a}$ are as usual the Pauli matrices and $\lambda^{a}$ the Gell-Mann matrices.

A quantum field theory might have a Lagrangian that is invariant under a symmetry transformation, but it does not mean that the full theory is. In terms of path integrals, the measure also has to be invariant for the generating functional to be invariant, which is not guaranteed (see Refs. [11,12] for more details). Without going into technical details, the chiral transformation of an individual Weyl spinor will add to the Lagrangian a term

$$
\Delta \mathcal{L}_{a}=\alpha \sum_{b, c} g_{b} g_{c} \frac{\operatorname{tr}\left[T_{a}\left\{T_{b}, T_{c}\right\}\right]}{64 \pi^{2}} \epsilon^{\alpha \beta \mu \nu} F_{\alpha \beta}^{b} F_{\mu \nu}^{c}
$$

where the sum over Latin indices is over all generators of $S U(3)_{c} \times S U(2)_{L} \times U(1)_{Y}$, $F_{\mu \nu}^{b}$ represents a generic field strength and $g_{b}$ and $g_{c}$ are the gauge couplings constant associated with $T_{b}$ and $T_{c}$ respectively. This can be used to cancel the vacuum angles. The price to pay though is the introduction of a factor of $\exp \left(i \alpha T_{a}\right)$ (or its complex conjugate) in the Yukawa interactions. This translates to complex phases in Dirac masses once electroweak symmetry is broken.

For $S U(2)_{L}$, the left-handed and right-handed fermions that appear in the Yukawa interactions are charged differently. It is then possible to cancel their vacuum angle without introducing complex Dirac masses by an appropriate field redefinition 
of both left-handed and right-handed fermions. For example, redefining a single $Q_{L} \rightarrow \exp \left(-i \Theta_{L} / 3\right) Q_{L}$ will add to the Lagrangian a term

$$
\begin{aligned}
\Delta \mathcal{L} & =-\frac{g^{2} \Theta_{L}}{192 \pi^{2}} \operatorname{tr}\left[\mathbb{I}_{6 \times 6}\left\{\mathbb{I}_{3 \times 3} \otimes \frac{\sigma^{a}}{2}, \mathbb{I}_{3 \times 3} \otimes \frac{\sigma^{b}}{2}\right\}\right] \epsilon^{\alpha \beta \mu \nu} W_{\alpha \beta}^{a} W_{\mu \nu}^{b} \\
& =-\frac{g^{2} \Theta_{L}}{192 \pi^{2}} \operatorname{tr}\left[\mathbb{I}_{6 \times 6}\left(\mathbb{I}_{3 \times 3} \otimes\left\{\frac{\sigma^{a}}{2}, \frac{\sigma^{b}}{2}\right\}\right)\right] \epsilon^{\alpha \beta \mu \nu} W_{\alpha \beta}^{a} W_{\mu \nu}^{b} \\
& =-\frac{g^{2} \Theta_{L}}{192 \pi^{2}} \operatorname{tr}\left[\mathbb{I}_{6 \times 6}\left(\mathbb{I}_{3 \times 3} \otimes \frac{\mathbb{I}_{2 \times 2}}{2} \delta^{a b}\right)\right] \epsilon^{\alpha \beta \mu \nu} W_{\alpha \beta}^{a} W_{\mu \nu}^{b} \\
& =-\frac{g^{2} \Theta_{L}}{384 \pi^{2}} \operatorname{tr}\left[\mathbb{I}_{6 \times 6}\right] \epsilon^{\alpha \beta \mu \nu} W_{\alpha \beta}^{a} W_{\mu \nu}^{a} \\
& =-\frac{g^{2} \Theta_{L}}{64 \pi^{2}} \epsilon^{\alpha \beta \mu \nu} W_{\alpha \beta}^{a} W_{\mu \nu}^{a},
\end{aligned}
$$

where we used $\left\{\sigma^{a}, \sigma^{b}\right\}=2 \delta^{a b}$ and properties of the Kronecker product. This term cancels exactly the vacuum angle $\Theta_{L}$, but reintroduces a phase in the quark masses. Since the right-handed quarks are not charged under $S U(2)_{L}, D_{L}$ and $U_{L}$ can be used to reabsorb the phase of $\exp \left(i \Theta_{L} / 3\right)$ without reintroducing $\Theta_{L}$.

However, such a procedure is impossible for $S U(3)_{c}$. Leaving the masses of quarks real while multiplying $Q_{L}$ by a phase $\exp (i \alpha)$ requires multiplying $D_{L}$ and $U_{L}$ by $\exp (-i \alpha)$. The correction to the vacuum angle is then

$$
\begin{aligned}
\Delta \mathcal{L}= & \frac{\alpha g_{s}^{2}}{64 \pi^{2}}\left(\operatorname{tr}\left[\mathbb{I}_{6 \times 6}\left\{\frac{\lambda^{a}}{2} \otimes \mathbb{I}_{2 \times 2}, \frac{\lambda^{b}}{2} \otimes \mathbb{I}_{2 \times 2}\right\}\right]-\operatorname{tr}\left[\mathbb{I}_{3 \times 3}\left\{\frac{\lambda^{a *}}{2}, \frac{\lambda^{b *}}{2}\right\}\right]\right. \\
& \left.\quad-\operatorname{tr}\left[\mathbb{I}_{3 \times 3}\left\{\frac{\lambda^{a *}}{2}, \frac{\lambda^{b *}}{2}\right\}\right]\right) \epsilon^{\alpha \beta \mu \nu} G_{\alpha \beta}^{a} G_{\mu \nu}^{b} \\
= & \frac{\alpha g_{s}^{2}}{64 \pi^{2}}\left(\operatorname{tr}\left[\mathbb{I}_{6 \times 6}\left(\left\{\frac{\lambda^{a}}{2}, \frac{\lambda^{b}}{2}\right\} \otimes \mathbb{I}_{2 \times 2}\right)\right]-\operatorname{tr}\left[\mathbb{I}_{3 \times 3}\left\{\frac{\lambda^{a *}}{2}, \frac{\lambda^{b *}}{2}\right\}\right]\right. \\
& \left.\quad-\operatorname{tr}\left[\mathbb{I}_{3 \times 3}\left\{\frac{\lambda^{a *}}{2}, \frac{\lambda^{b *}}{2}\right\}\right]\right) \epsilon^{\alpha \beta \mu \nu} G_{\alpha \beta}^{a} G_{\mu \nu}^{b} \\
= & \frac{\alpha g_{s}^{2}}{64 \pi^{2}}\left(\operatorname{tr}\left[\mathbb{I}_{6 \times 6}\right]-\operatorname{tr}\left[\mathbb{I}_{3 \times 3}\right]-\operatorname{tr}\left[\mathbb{I}_{3 \times 3}\right]\right) \frac{1}{3} \delta^{a b} \epsilon^{\alpha \beta \mu \nu} G_{\alpha \beta}^{a} G_{\mu \nu}^{b} \\
= & \frac{\alpha g_{s}^{2}}{192 \pi^{2}}(6-3-3) \epsilon^{\alpha \beta \mu \nu} G_{\alpha \beta}^{a} G_{\mu \nu}^{a}=0,
\end{aligned}
$$


where we used $\left\{\lambda_{a}, \lambda_{b}\right\}=2 d_{a b c} \lambda_{c}+\frac{4}{3} \delta^{a b}$ with $d_{a b c}$ a set of irrelevant constants. The QCD vacuum angle therefore cannot be rotated away and is physical. However, it is constrained to be very small by measurements of the neutron electric dipole moment. This will be discussed further in Section 2.4. We mention that the QCD vacuum angle breaks $C P$-invariance. It is also a divergence and is only expected to have non-perturbative effects.

Finally, the vacuum angle of $U(1)_{Y}$ is irrelevant for topological reasons beyond the scope of this thesis (see Ref. [13] for example).

\subsubsection{Anomaly cancellation}

Another crucial property of the Standard Model is that it does not have gauge anomalies, which is what is meant when the Standard Model is said to be anomaly free. This means that the terms added to the Lagrangian by Eq. (2.24) cancel when a complete gauge transformation is performed. This will be true as long as the following quantity is zero

$$
\mathcal{A}_{a b c}=\sum_{\text {fermions }} \operatorname{tr}\left[T_{a}\left\{T_{b}, T_{c}\right\}\right],
$$

where the sum is over all fermions. It is a simple but tedious calculation to show that all $\mathcal{A}_{a b c}$ 's cancel in the Standard Model. For example, consider the case of the three generators of weak hypercharge. The calculation gives

$$
\begin{aligned}
\mathcal{A}_{Y Y Y} & =6\left(\left(\frac{1}{6}\right)^{3} \operatorname{tr}\left[\mathbb{I}_{6 \times 6}\right]+\left(-\frac{2}{3}\right)^{3} \operatorname{tr}\left[\mathbb{I}_{3 \times 3}\right]+\left(\frac{1}{3}\right)^{3} \operatorname{tr}\left[\mathbb{I}_{3 \times 3}\right]+\left(-\frac{1}{2}\right)^{3} \operatorname{tr}\left[\mathbb{I}_{2 \times 2}\right]+1^{3}\right) \\
& =6\left(\frac{1}{36}-\frac{8}{9}+\frac{1}{9}-\frac{1}{4}+1\right) \\
& =0
\end{aligned}
$$


The case of the weak hypercharge and two $S U(2)_{L}$ generators gives

$$
\begin{aligned}
\mathcal{A}_{Y L^{a} L^{b}} & =3\left(\frac{1}{6} \operatorname{tr}\left[\mathbb{I}_{6 \times 6}\left\{\mathbb{I}_{3 \times 3} \otimes \frac{\sigma^{a}}{2}, \mathbb{I}_{3 \times 3} \otimes \frac{\sigma^{b}}{2}\right\}\right]-\frac{1}{2} \operatorname{tr}\left[\mathbb{I}_{2 \times 2}\left\{\frac{\sigma^{a}}{2}, \frac{\sigma^{b}}{2}\right\}\right]\right) \\
& =3\left(\frac{1}{6} \operatorname{tr}\left[\mathbb{I}_{6 \times 6}\left(\mathbb{I}_{3 \times 3} \otimes\left\{\frac{\sigma^{a}}{2}, \frac{\sigma^{b}}{2}\right\}\right)\right]-\frac{1}{2} \operatorname{tr}\left[\mathbb{I}_{2 \times 2}\left\{\frac{\sigma^{a}}{2}, \frac{\sigma^{b}}{2}\right\}\right]\right) \\
& =\frac{3 \delta^{a b}}{2}\left(\frac{1}{6} \operatorname{tr}\left[\mathbb{I}_{6 \times 6}\left(\mathbb{I}_{3 \times 3} \otimes \mathbb{I}_{2 \times 2}\right)\right]-\frac{1}{2} \operatorname{tr}\left[\mathbb{I}_{2 \times 2}\left(\mathbb{I}_{2 \times 2}\right)\right]\right) \\
& =\frac{3 \delta^{a b}}{2}\left(\frac{1}{6} \operatorname{tr}\left[\mathbb{I}_{6 \times 6}\right]-\frac{1}{2} \operatorname{tr}\left[\mathbb{I}_{2 \times 2}\right]\right)=\frac{3 \delta^{a b}}{2}(1-1)=0 .
\end{aligned}
$$

These cancellations are non-trivial and the fact that they all take place is due to a subtle interplay between quarks and leptons. The Lagrangian in Eq. (2.9) only requires $Y_{L_{L}}+Y_{E_{L}}=Y_{H}, Y_{Q_{L}}+Y_{U_{L}}=-Y_{H}$ and $Y_{Q_{L}}+Y_{D_{L}}=Y_{H}$, which is an undetermined set of equations. It would therefore have been possible to assign the hypercharges differently such that only the $U(1)_{Y}$ gauge interactions differ. In general, the theory would have a gauge anomaly. This would be disastrous as the presence of a chiral anomaly leads to inconsistencies [14]. The fact that the Standard Model is anomaly free is certainly an impressive feature.

As a side note, these miraculous cancellations are a motivation for Grand Unified Theories (GUT). These are theories in which the Standard Model groups unify in a simple (semisimple) group. In fact, the inclusion of three generations of right-handed neutrinos allows all fermions from one generation to be grouped into a single spinorial representation of $S O(10)$. Since this group is anomaly free [15] and the right-handed neutrino would have to be a singlet under all SM gauge groups, the Standard Model is anomaly free as well. 


\subsubsection{Baryon and lepton numbers}

Finally, the Lagrangian of the Standard Model is invariant under baryon and lepton transformations even after symmetry breaking. A baryon transformation means multiplying all fields by $\exp \left(i \alpha B_{i}\right)$, where $\alpha$ parametrizes the transformation and $B_{i}$ is the baryon number of a field. The fields $Q_{L}^{m}, U_{R}^{m}$ and $D_{R}^{m}$ have baryon number $+1 / 3$, while all other fields have 0 . A lepton transformation is similar but with $B_{i}$ replaced by the lepton number $L_{i}$. The fields $L_{L}^{m}$ and $E_{R}^{m}$ have lepton number +1 , while all other fields have 0 . Classically, this leads to conservation of baryon and lepton numbers. Since all interactions involving leptons can be made flavour preserving, lepton numbers can be defined for individual generations, i.e. electron, muon and tau numbers. However, both baryon and lepton symmetries are anomalous. This means that baryon and lepton number conservation are expected to be violated nonpertubatively, though this effect is very small. We will come back to this in Chapter 8. Interestingly, $B-L$ is anomaly free and therefore conserved.

\subsection{Shortcomings of the Standard Model}

Despite all of its successes, the Standard Model is not without a few shortcomings. Some of them are experimental, while others are more of a theoretical nature. We list a few of them here.

Neutrino masses. Perhaps the most tangible evidence that the Standard Model needs to be extended is the fact that neutrino masses are non-zero. Though these masses are not known yet, studies of neutrino oscillation via solar, atmospheric, accelerator and reactor experiments have allowed us to determine their mass differences and reconstruct the PMNS matrix. Fortunately, only minimal extensions of the 
Standard Model are required to accommodate such masses.

Dark matter. Another experimental shortcoming is that it is now known that baryonic matter only constitutes about $5 \%$ of the content of the Universe. Roughly $25 \%$ is made up of dark matter, whose nature is unknown and which must interact only weakly with the Standard Model. The $70 \%$ left over is known as dark energy and its nature is also unknown.

Baryogenesis. On a related note, the Universe displays an asymmetry between matter and antimatter. Baryons make up about $5 \%$ of the mass of the Universe, while antibaryons only represent a negligible fraction. This asymmetry cannot be explained by the Standard Model alone. We will discus this issue more deeply in Chapter 8.

Strong $C P$ problem. As mentioned in Section 2.3, the Standard Model contains a parameter $\Theta_{s}$ called the QCD vacuum angle that breaks $C P$-invariance. Experimental constraints from the neutron electric dipole moment constrain this parameter to be $\left|\Theta_{s}\right|<10^{-10}[16]$. However, this term is the coefficient of an operator which happens to be a divergence. From this, it is not difficult to prove that $\Theta_{s}$ is actually defined modulo $2 \pi$, hence the angle terminology. As such, one would expect $\Theta_{s}$ to be of order 1 .

In addition, the Yukawa interactions can be complex in the gauge basis, which leads to complex masses. This is usually dealt with by field redefinition of the fermions. Since such transformations are anomalous in general, this will add to the QCD vacuum angle a term suppressed by a single loop factor proportional to the phase of $\operatorname{det}(M)$, where $M$ is the mass matrix of quarks. Since the phases in the Yukawa interactions can perfectly be of order 1, one would therefore expect the QCD vacuum angle to be at least of order $\alpha_{S}=g_{s}^{2} /(4 \pi)$, which is much larger than the current constraints. 
The fact that the QCD vacuum angle is constrained to be so small while one would expect it to be close to unity is not fully understood yet. There are models that can accommodate a very small or zero value, such as the introduction of axions in the context of the Peccei-Quinn model $[17,18]$.

Hierarchy problem. The problem of the Standard Model that will be the main focus of this thesis is the hierarchy problem. It goes as follows.

The Standard Model is an effective theory and is expected to eventually break down as higher energies are reached. The scale of new physics serves as an effective cutoff for the theory.

All parameters in the Standard Model receive radiative corrections. The parameter that receives the largest corrections is the mass of the Higgs squared $\mu^{2}$. Such corrections depend quadratically on the cutoff.

One scale at which we are certain the Standard Model becomes invalid is the Planck scale $\left(10^{19} \mathrm{GeV}\right)$, where gravity becomes of the same strength as the other forces. If the Planck scale is indeed the cutoff of the Standard Model, the corrections to the Higgs mass are then only slightly smaller than the Planck scale. To obtain an effective Higgs mass close to the electroweak scale requires a tuning between the bare parameters and the radiative corrections of about 1 part in $10^{32}$. It would be an understatement to say that this amount of tuning seems unnatural. This is what is known as the hierarchy problem. 


\section{Chapter 3}

\section{Supersymmetry}

Supersymmetry (SUSY) in its simplest form is a theory that posits the existence of a superpartner of different spin for every particle of the Standard Model. Interestingly, it can be seen as the only viable extension of the Poincaré group, the Lie group which governs the symmetries of spacetime in special relativity. Indeed, Coleman and Mandula [19] proved long ago that, under physically reasonable assumptions, the Poincaré group and internal symmetries can only be combined in a larger Lie group as a direct product of the Poincaré group itself and a Lie group of internal symmetries. The resulting algebra is thus a direct sum of the Poincaré algebra and an internal symmetry algebra, i.e. that Poincaré and internal symmetries are each associated to an ideal and therefore that elements of the two different algebras commute with respect to one another. In less mathematical terms, spacetime symmetries and internal symmetries do not mix. A loophole is to promote the Lie algebra to a Lie superalgebra, a (graded) algebra that also accepts anticommutation relations. Furthermore, the only acceptable superalgebras are the different extended supersymmetric algebras, which interchange components within a multiplet of fields of different spins. This result is

known as the Haag-Lopuszanski-Sohnius theorem [20]. The simplest realization of 

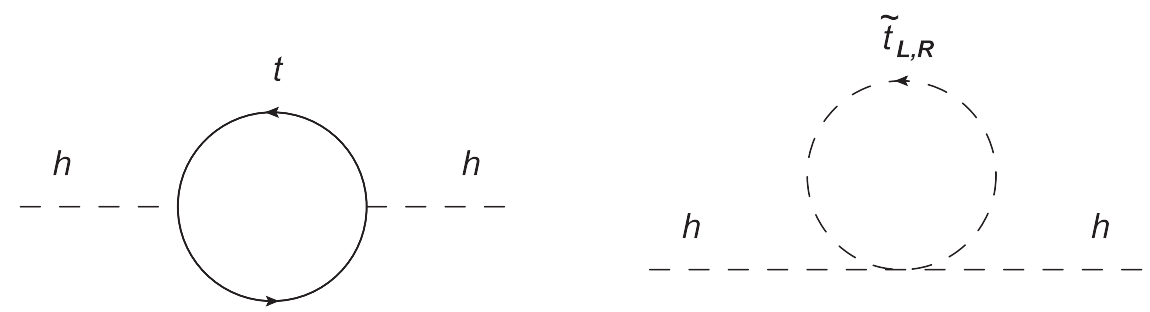

Figure 3.1: Contribution of the top and its partners to the Higgs mass. The quadratic divergences cancel.

Supersymmetry is in its $\mathcal{N}=1$ form, for which each SM particle is partnered to a particle whose spin differs by $1 / 2$. Realizations of Supersymmetry with $\mathcal{N}>1$ will not be discussed in this work.

In addition to the attractive nature of the Haag-Lopuszanski-Sohnius theorem, there are several theoretical and experimental motivations for SUSY. Perhaps most importantly, it is a solution to the hierarchy problem. An alternative way to think of the hierarchy problem is that it arises because of the lack of protection of the Higgs mass. The supersymmetrization of the Standard Model elegantly solves this problem. Indeed, fermion masses break a chiral symmetry that would otherwise be present in the Standard Model (up to irrelevant topological effects related to the chiral anomaly). This symmetry insures that the fermion masses are protected against quadratic divergences. By relating fermions and bosons, Supersymmetry transmits this protection to the Higgs mass. From a more practical point of view, the superpartners introduce new radiative corrections whose quadratic divergences cancel exactly those of the Standard Model. This is illustrated in Fig. 3.1 in the case of the top quark.

Furthermore, supersymmetric extensions of the Standard Model can easily be made to accommodate an extra symmetry. The best example of this is $R$-parity, a $\mathbb{Z}_{2}$ symmetry under which SM particles are even and their partners odd. This 
symmetry insures that the lightest superpartner (LSP) is stable. The LSP can then be made relatively easily into a viable dark matter candidate. Such particles fall into the category of weakly interacting massive particles (WIMP).

Also of great importance is that the introduction of superpartners not too far removed from the electroweak scale leads to gauge coupling unification. These new particles modify the evolution of gauge coupling constants just enough for them to unify at around $10^{16} \mathrm{GeV}$. Such a behavior is expected if the Standard Model is to be embedded in a simple group, as in most Grand Unified Theories.

This chapter summarizes the main results from Supersymmetry that will be useful for this work. The Supersymmetry algebra and its differential representation are first discussed. The different types of superfields used in this thesis are then presented. Using these, supersymmetric Lagrangians are constructed. The Minimal Supersymmetric Standard Model (MSSM), the simplest supersymmetric extension of the Standard Model, is then discussed. This model must however be supplemented by soft terms that explicitly break Supersymmetry but do not reintroduce large corrections to the Higgs mass. These are generated by mediation mechanisms and we give three pertinent examples: Gravity, gauge and anomaly mediation. See Appendix B for an introduction to Grassmann variables and Appendix C for an explanation of the van der Waerden notation.

As Supersymmetry is a well documented subject, all results in this chapter are well-known and we draw inspiration from several sources. We also refer to them for more details. The notation is that of Ref. [21] with slight modifications. The results of Section 3.4.1 can be found in Ref. [22]. Section 3.5 draws its inspiration from Refs. [22] and [23], but all results are rewritten to be more in line with the notation and spirit of this thesis. 


\subsection{The SUSY algebra}

We take the Supersymmetry algebra as the starting point of our explanation of Supersymmetry. In general, it involves $\mathcal{N}$ generators that parametrize the supersymmetric transformations. In the $\mathcal{N}=1$ case, the single SUSY generator can be written as a complex Weyl spinor $Q$. This generator has non-trivial (anti-)commutation relations with the generators of the Lorentz group $M_{\mu \nu}$ and of spacetime translations $P_{\mu}$, i.e. the Poincaré algebra. The part of the algebra involving $Q$ is given by

$$
\begin{array}{rlrl}
{\left[Q_{\alpha}, P_{\mu}\right]} & =0, & {\left[\bar{Q}_{\dot{\alpha}}, P_{\mu}\right]} & =0, \\
{\left[Q_{\alpha}, M_{\mu \nu}\right]} & =\left(\sigma_{\mu \nu}\right)_{\alpha}{ }^{\beta} Q_{\beta}, & {\left[\bar{Q}^{\dot{\alpha}}, M_{\mu \nu}\right]} & =\left(\bar{\sigma}_{\mu \nu}\right)^{\dot{\alpha}}{ }_{\dot{\beta}} \bar{Q}^{\dot{\beta}}, \\
\left\{Q_{\alpha}, Q_{\beta}\right\} & =0, & \left\{\bar{Q}_{\dot{\alpha}}, \bar{Q}_{\dot{\beta}}\right\}=0, \\
\left\{Q_{\alpha}, \bar{Q}_{\dot{\beta}}\right\} & =\left(\sigma^{\mu}\right)_{\alpha \dot{\beta}} P_{\mu} . &
\end{array}
$$

This algebra is completed by the Poincaré algebra,

$$
\begin{aligned}
{\left[P_{\mu}, P_{\nu}\right] } & =0, \quad\left[M_{\mu \nu}, P_{\alpha}\right]=i\left(\eta_{\nu \alpha} P_{\mu}-\eta_{\mu \alpha} P_{\nu}\right) \\
{\left[M_{\mu \nu}, M_{\alpha \beta}\right] } & =i\left(\eta_{\mu \beta} M_{\nu \alpha}-\eta_{\mu \alpha} M_{\nu \beta}-\eta_{\nu \beta} M_{\mu \alpha}+\eta_{\nu \alpha} M_{\mu \beta}\right) .
\end{aligned}
$$

The SUSY algebra can be assigned a differential representation by having it act on superfields. These latter are fields that, in addition to spacetime coordinates, also depend on Grassmann coordinates. For $\mathcal{N}=1$ SUSY, the new Grassmann coordinates are encoded in a complex left-handed Weyl spinor $\theta$. Since Grassmann variables anticommute, the Taylor expansion of a superfield in terms of $\theta$ terminates. The coefficients of the different powers of $\theta$ that appear in this expansion are interpreted as 
the physical fields. The SUSY algebra can then be represented as

$$
\begin{aligned}
\hat{P}_{\mu} & =i \partial_{\mu}, & \hat{M}_{\mu \nu} & =i\left(x_{\mu} \partial_{\nu}-x_{\nu} \partial_{\mu}\right), \\
\mathcal{Q}_{\alpha} & =i \partial_{\alpha}-\frac{1}{2}\left(\sigma^{\mu}\right)_{\alpha \dot{\beta}} \bar{\theta}^{\dot{\beta}} \partial_{\mu}, & \overline{\mathcal{Q}}_{\dot{\alpha}} & =-i \bar{\partial}_{\dot{\alpha}}+\frac{1}{2} \theta^{\beta}\left(\sigma^{\mu}\right)_{\beta \dot{\alpha}} \partial_{\mu} .
\end{aligned}
$$

In addition to transforming under the Poincaré group and possibly gauge groups, a superfield also transforms under Supersymmetry. For example, a scalar superfield will transform under a Supersymmetry transformation parametrized by $\zeta$ as

$$
\Phi \rightarrow \exp (-i \zeta \cdot \mathcal{Q}-i \bar{\zeta} \cdot \overline{\mathcal{Q}}) \Phi
$$

The parameter $\zeta$ is taken to be a constant left-handed Weyl spinor. Making Supersymmetry local leads to Supergravity. Note that $P_{\mu} P^{\mu}$ commutes with every generator. As such, the mass of a particle and its superpartner will be equal as long as Supersymmetry is unbroken.

\subsection{Superfields}

The most general scalar superfield for $\mathcal{N}=1$ Supersymmetry is

$$
\begin{aligned}
\Phi(x)= & A(x)+\theta \cdot \alpha(x)+\bar{\theta} \cdot \bar{\beta}(x)+\theta \cdot \theta B(x)+\bar{\theta} \cdot \bar{\theta} C(x)+\theta \sigma^{\mu} \bar{\theta} V_{\mu}(x) \\
& +\theta \cdot \theta \bar{\theta} \cdot \bar{\gamma}(x)+\bar{\theta} \cdot \bar{\theta} \theta \cdot \zeta(x)+\theta \cdot \theta \bar{\theta} \cdot \bar{\theta} D(x) .
\end{aligned}
$$

The number of degrees of freedom contained in this superfield is very large and building a theory in terms of such superfields is not usually the approach taken as they lead to reducible representations of SUSY. Superfields must therefore be constrained by either fixing some terms to zero or by relating terms to each other. The two most common examples of this and the only two types of superfields that we will use are 
chiral superfields and vector superfields. These are presented here.

\subsubsection{Chiral Superfields}

Generally speaking, it is insufficient to simply impose an arbitrary condition on a given superfield. This constraint has to be preserved under the supersymmetric transformation of Eq. (3.4). Assume a superfield $\Phi$ is constrained by imposing $\mathcal{D}_{\alpha} \Phi=0$, where $\mathcal{D}_{\alpha}$ is a linear operator taking the form of a Weyl spinor and which anticommutes with itself. This constraint will remain satisfied under supersymmetric transformations as long as

$$
\left\{Q_{\alpha}, \mathcal{D}_{\beta}\right\}=0, \quad\left\{\bar{Q}_{\dot{\alpha}}, \mathcal{D}_{\beta}\right\}=0 .
$$

It is easy to verify that the following operators satisfy these constraints

$$
D_{\alpha}=\partial_{\alpha}-\frac{i}{2}\left(\sigma^{\mu}\right)_{\alpha \dot{\beta}} \bar{\theta}^{\dot{\beta}} \partial_{\mu}, \quad \bar{D}_{\dot{\alpha}}=\bar{\partial}_{\dot{\alpha}}-\frac{i}{2} \theta^{\gamma}\left(\sigma^{\mu}\right)_{\gamma \dot{\alpha}} \partial_{\mu} .
$$

These operators are referred to as supersymmetric covariant derivatives. A field $\Phi$ is said to be left-chiral if $\bar{D}_{\dot{\alpha}} \Phi=0$ and right-chiral if $D_{\alpha} \Phi=0$. Most authors choose to work only with left-chiral superfields and refer to such superfields simply as chiral superfields. We will adopt this convention as well.

To express a chiral superfield $\Phi$ in terms of its components, one starts by expanding it in terms of the Grassmann variable $\theta$

$$
\Phi=\phi(y)+\theta \cdot \chi(y)+\frac{1}{2} \theta \cdot \theta F(y)
$$

where $y$ is assumed a function of $\bar{\theta}$. The superfield $\Phi$ will then be chiral as long as 
$\bar{D}_{\dot{\alpha}} y^{\mu}=0$. This is satisfied by

$$
y^{\mu}=x^{\mu}-\frac{i}{2} \theta \sigma^{\mu} \bar{\theta}
$$

Expanding $\Phi$ then leads to

$$
\Phi=\phi(x)-\frac{i}{2} \theta \sigma^{\mu} \bar{\theta} \partial_{\mu} \phi(x)-\frac{1}{16} \theta \cdot \theta \bar{\theta} \cdot \bar{\theta} \square \phi(x)+\theta \cdot \chi(x)-\frac{i}{2} \theta \sigma^{\mu} \bar{\theta} \theta \cdot \partial_{\mu} \chi(x)+\frac{1}{2} \theta \cdot \theta F(x) .
$$

The physical content of a chiral superfield is a scalar $\phi$ and a left-handed Weyl spinor $\chi$. The field $F$ is non-dynamical and can be solved for exactly in terms of scalar fields. It is known as an auxiliary field.

A result that will be very useful later on is that the product of two chiral superfields is also a chiral superfield. This result is easily verified by proving that supersymmetric covariant derivatives obey a chain derivative rule. However, the conjugate of a chiral superfield is not a chiral superfield. One can then obtain more complicated chiral superfields by multiplying together chiral superfields but not their conjugates, i.e. a holomorphic function of the superfields.

Also of importance is how component fields transform under a supersymmetric transformation. Performing a SUSY transformation as in Eq. (3.4) parametrized by $\zeta$ on a gauge singlet chiral superfield leads to

$$
\begin{aligned}
& \phi \rightarrow \phi+\zeta \cdot \chi, \\
& \chi \rightarrow \chi-i \sigma^{\mu} \bar{\zeta} \partial_{\mu} \phi+\zeta F, \\
& F \rightarrow F-i \bar{\zeta} \bar{\sigma}^{\mu} \partial_{\mu} \chi .
\end{aligned}
$$

If the superfield were to be charged under some gauge groups, the transformation laws would be modified slightly (see the next section). The important result is that, in the 
case of a gauge singlet, this transformation only adds a divergence to $F$. This was to be expected. A small supersymmetric transformation replaces a component field by a linear combination of different component fields and their derivatives. Since $\theta$ must have units of [Energy $]^{-1 / 2}, F$ has units [Energy $]^{2}$ which is higher than all other fields. The corrections it receives must therefore be a divergence. A very important result is then that a Lagrangian that consists of the $F$ part of a gauge singlet holomorphic function of chiral superfields leads to a supersymmetric action. This will be used abundantly in creating supersymmetric theories.

\subsubsection{Vector superfields}

The number of degrees of freedom of the generic superfield in Eq. (3.5) can also be

reduced by imposing $\mathcal{V}^{\dagger}=\mathcal{V}$. Such a superfield is referred to as a vector superfield. A convenient parametrization of a vector superfield is

$$
\begin{aligned}
\mathcal{V} & =C(x)+\frac{i}{\sqrt{2}} \theta \cdot \rho(x)-\frac{i}{\sqrt{2}} \bar{\theta} \cdot \bar{\rho}(x)+\frac{i}{4} \theta \cdot \theta(M(x)+i N(x)) \\
& -\frac{i}{4} \bar{\theta} \cdot \bar{\theta}(M(x)-i N(x))+\frac{1}{2} \theta \sigma^{\mu} \bar{\theta} A_{\mu}(x)+\frac{1}{2 \sqrt{2}} \theta \cdot \theta\left(\bar{\theta} \cdot \bar{\lambda}(x)+\frac{1}{2} \bar{\theta} \bar{\sigma}^{\mu} \partial_{\mu} \rho(x)\right) \\
& +\frac{1}{2 \sqrt{2}} \bar{\theta} \cdot \bar{\theta}\left(\theta \cdot \lambda(x)-\frac{1}{2} \theta \sigma^{\mu} \partial_{\mu} \bar{\rho}(x)\right)-\frac{1}{8} \theta \cdot \theta \bar{\theta} \cdot \bar{\theta}\left(D(x)+\frac{1}{2} \square C(x)\right),
\end{aligned}
$$

where $C, M, N, D$ and $A_{\mu}$ are real fields. In the MSSM and all supersymmetric models considered in this work, it will be possible to simplify this expression further. Indeed, matter fields will be embedded in chiral superfields and gauge fields in vector superfields. Consider a chiral superfield $\Phi$ charged under an abelian group. Under gauge transformation, this chiral superfield transforms as

$$
\Phi \rightarrow e^{-2 i q \Lambda} \Phi
$$


where $q$ is the product of the gauge coupling constant $g$ of the group and the charge of the field and $\Lambda$ is a superfield that parametrizes the transformation. For $\Phi$ to remain a chiral superfield, $\Lambda$ must also be a chiral superfield. Kinetic terms will result from terms of the form

$$
\Phi^{\dagger} e^{2 q \mathcal{V}} \Phi
$$

where $\mathcal{V}$ is a vector superfield associated with the gauge group. For this term to be gauge invariant, $\mathcal{V}$ must transform as

$$
\begin{aligned}
\mathcal{V} & \rightarrow \mathcal{V}+i\left(\Lambda-\Lambda^{\dagger}\right) \\
& \rightarrow \mathcal{V}+i\left(\phi_{\Lambda}-\phi_{\Lambda}^{\dagger}\right)+i\left(\theta \cdot \chi_{\Lambda}-\bar{\theta} \cdot \bar{\chi}_{\Lambda}\right)+\frac{i}{2} \theta \cdot \theta F_{\Lambda}-\frac{i}{2} \bar{\theta} \cdot \bar{\theta} F_{\Lambda}^{\dagger} \\
& +\frac{1}{2} \theta \sigma^{\mu} \bar{\theta} \partial_{\mu}\left(\phi_{\Lambda}+\phi_{\Lambda}^{\dagger}\right)+\frac{1}{4}\left(\theta \cdot \theta \bar{\theta} \bar{\sigma}^{\mu} \partial_{\mu} \chi_{\Lambda}-\bar{\theta} \cdot \bar{\theta} \theta \sigma^{\mu} \partial_{\mu} \bar{\chi}_{\Lambda}\right) \\
& -\frac{i}{16} \theta \cdot \theta \bar{\theta} \cdot \bar{\theta} \square\left(\phi_{\Lambda}-\phi_{\Lambda}^{\dagger}\right),
\end{aligned}
$$

where Eq. (3.10) was used. Under such transformations, the different components of Eq. (3.12) transform as

$$
\begin{array}{ccc}
C \rightarrow C+i\left(\phi_{\Lambda}-\phi_{\Lambda}^{\dagger}\right), & \rho \rightarrow \rho+\sqrt{2} \chi_{\Lambda}, & M+i N \rightarrow M+i N+2 F_{\Lambda}, \\
A_{\mu} \rightarrow A_{\mu}+\partial_{\mu}\left(\phi_{\Lambda}+\phi_{\Lambda}^{\dagger}\right), & \lambda \rightarrow \lambda, & D \rightarrow D .
\end{array}
$$

This gauge freedom can be used to remove $C, \rho, M$ and $N$ from Eq. (3.12). The vector superfield $\mathcal{V}$ can then be expressed as

$$
\mathcal{V}=\frac{1}{2} \theta \sigma^{\mu} \bar{\theta} A_{\mu}+\frac{1}{2 \sqrt{2}} \theta \cdot \theta \bar{\theta} \cdot \bar{\lambda}+\frac{1}{2 \sqrt{2}} \bar{\theta} \cdot \bar{\theta} \theta \cdot \lambda-\frac{1}{8} \theta \cdot \theta \bar{\theta} \cdot \bar{\theta} D
$$

This is known as the Wess-Zumino gauge. The physical fields are then a gauge boson and the corresponding gaugino. The field $D$ is non-dynamical and can be solved for 
in terms of the scalar fields. There is a residual symmetry,

$$
A_{\mu} \rightarrow A_{\mu}+2 \partial_{\mu} \operatorname{Re}\left\{\phi_{\Lambda}\right\} .
$$

This corresponds to the usual gauge invariance of non-supersymmetric gauge theories. The same result can easily be obtained for a non-abelian gauge group,

$$
\mathcal{V}^{i}=\frac{1}{2} \theta \sigma^{\mu} \bar{\theta} A_{\mu}^{i}+\frac{1}{2 \sqrt{2}} \theta \cdot \theta \bar{\theta} \cdot \bar{\lambda}^{i}+\frac{1}{2 \sqrt{2}} \bar{\theta} \cdot \bar{\theta} \theta \cdot \lambda^{i}-\frac{1}{8} \theta \cdot \theta \bar{\theta} \cdot \bar{\theta} D^{i},
$$

where gauge bosons, gauginos and auxiliary fields now come in multiplets.

A very important result is that the $D$ part of a vector superfield gauge singlet transforms as a divergence. This can be proven by the same argument as in the last part of Section 3.2.1. The $D$ part of a vector superfield that is a gauge singlet will therefore be invariant under both Supersymmetry and gauge symmetry. This will be used abundantly to build supersymmetric Lagrangians.

Though this result is not crucial to the rest of the thesis, we note for completeness that a vector superfield transforms under Supersymmetry as

$$
\begin{aligned}
A^{i \mu} & \rightarrow A^{i \mu}-\frac{1}{\sqrt{2}}\left(\bar{\zeta} \bar{\sigma}^{\mu} \lambda^{i}+\bar{\lambda} \bar{\sigma}^{\mu} \zeta\right), \\
\lambda^{i} & \rightarrow \lambda^{i}-\frac{i}{2 \sqrt{2}} F_{\mu \nu}^{i} \sigma^{\mu} \bar{\sigma}^{\mu} \zeta-\frac{1}{\sqrt{2}} D^{i} \zeta, \\
D^{i} & \rightarrow D^{i}+\frac{i}{\sqrt{2}} \bar{\zeta} \bar{\sigma}^{\mu} D_{\mu} \lambda^{i}-\frac{i}{\sqrt{2}}\left(D_{\mu} \bar{\lambda}^{i}\right) \bar{\sigma}^{\mu} \zeta .
\end{aligned}
$$

Note that $D$ indeed transforms like a divergence for a gauge singlet. In addition, some components of the vector superfields will appear in the transformation rules of the chiral superfields if those are charged under the corresponding gauge groups. Suppose a chiral superfield $\Phi$ which is charged under a single gauge group whose components 
are denoted $\Phi^{a}$. Then, Eq. (3.11) becomes

$$
\begin{aligned}
& \phi^{a} \rightarrow \phi^{a}+\zeta \cdot \chi^{a}, \\
& \chi^{a} \rightarrow \chi^{a}-i \sigma^{\mu} \bar{\zeta} D_{\mu} \phi^{a}+\zeta F^{a}, \\
& F^{a} \rightarrow F^{a}-i \bar{\zeta} \bar{\sigma}^{\mu} D_{\mu} \chi^{a}+\sqrt{2} g\left(\tau^{i} \phi\right)^{a} \bar{\lambda}^{i} \cdot \bar{\zeta}
\end{aligned}
$$

where the $\tau^{i}$ s are the generators of the gauge group for the representation under which $\Phi$ transforms. The only modification for a field charged under multiple gauge groups would be a trivial extension of the last line of Eq. (3.21).

\subsection{Supersymmetric Lagrangians}

We now have all the necessary tools to formulate a supersymmetric theory. The Lagrangian that describes such a theory usually consists of three parts. The first one is associated with the kinetic terms of the chiral superfields, while the second is associated with those of the gauge superfields. The third part is related to the superpotential, which will be described in what follows. In addition, so-called soft terms can be added to the Lagrangian. These terms break Supersymmetry explicitly but do not reintroduce quadratic divergences. We now proceed to explain how to build a supersymmetric theory and the resulting potential but postpone the discussion of soft terms to Section 3.4.

\subsubsection{Chiral superfield kinetic terms}

To obtain a supersymmetric Lagrangian that contains the kinetic terms of the chiral superfields, we start by considering a chiral superfield $\Phi$ of the form of Eq. (3.8) that is only charged under a single abelian gauge group. The vector superfield associated 
with the gauge group is labelled $\mathcal{V}$ and defined as in Eq. (3.17). First, note that the following term is a vector superfield,

$$
\Phi^{\dagger} e^{2 q \mathcal{V}} \Phi
$$

where $q$ is once again the product of the gauge coupling of the abelian group times the charge of the field. As explained before, the $D$ part of this term leads to an action that is supersymmetric and gauge invariant. A simple but tedious calculation leads to

$$
\left.\Phi^{\dagger} e^{2 q \mathcal{V}} \Phi\right|_{D}=\left(D_{\mu} \phi\right)^{\dagger}\left(D^{\mu} \phi\right)+i \bar{\chi} \bar{\sigma}^{\mu} D_{\mu} \chi+F^{\dagger} F-q \phi^{\dagger} \phi D-\left(\sqrt{2} q \phi^{\dagger} \chi \cdot \lambda+\text { h.c. }\right)
$$

where $D_{\mu}=\partial_{\mu}+i q A_{\mu}$ and the $\left.\right|_{D}$ means that we take the $D$ component of the resulting vector superfield. This gives the properly normalized kinetic terms for complex scalars and Weyl spinors. In addition, it contains the information on the interactions between the different components of the vector superfields and the chiral superfields. In the non-abelian case, this generalizes to

$$
\begin{aligned}
\left.\Phi^{\dagger} e^{2 q \mathcal{V}} \Phi\right|_{D} & =\left(D_{\mu} \phi\right)^{\dagger}\left(D^{\mu} \phi\right)+i \bar{\chi} \bar{\sigma}^{\mu} D_{\mu} \chi+F^{\dagger} F-q \phi^{\dagger} \tau^{i} \phi D^{i} \\
& -\left(\sqrt{2} q \phi^{\dagger} \tau^{i} \chi \cdot \lambda^{i}+\text { h.c. }\right)
\end{aligned}
$$

where the $\tau^{i}$ 's are representations of the Lie algebra of the group and $D_{\mu}=\partial_{\mu}+i q \tau^{i} A_{\mu}^{i}$. The extension to chiral superfields that are charged under multiple gauge groups is trivial. 


\subsubsection{Vector superfield kinetic terms}

To obtain the kinetic terms of the components of a vector superfield, we begin by examining the abelian case. It is useful to define the superfield strength by

$$
\mathcal{W}_{\alpha}=\bar{D} \cdot \bar{D} D_{\alpha} \mathcal{V}
$$

It is easily verified that $\mathcal{W}_{\alpha}$ is a chiral superfield. A tedious but straightforward calculation leads to

$$
\mathcal{W}_{\alpha}=\sqrt{2} \lambda_{\alpha}(y)-D(y) \theta_{\alpha}-F_{\mu \nu}(y)\left(\sigma^{\mu \nu}\right)_{\alpha}^{\beta} \theta_{\beta}+\frac{i}{\sqrt{2}} \theta \cdot \theta\left(\sigma^{\mu}\right)_{\alpha \dot{\beta}} \partial_{\mu} \bar{\lambda}^{\dot{\beta}}(y)
$$

where $y^{\mu}$ is defined as in Eq. (3.9). Taking the $F$ term of the contraction $\mathcal{W}^{\alpha} \mathcal{W}_{\alpha} / 8+$ h.c. leads to a SUSY invariant Lagrangian

$$
\left.\frac{1}{8}\left(\mathcal{W}^{\alpha} \mathcal{W}_{\alpha}\right)\right|_{F}+\text { h.c. }=\frac{1}{2} D^{2}+i \bar{\lambda} \bar{\sigma}^{\mu} \partial_{\mu} \lambda-\frac{1}{4} F_{\mu \nu} F^{\mu \nu}
$$

This leads to the correct field strength for the gauge boson and the correct kinetic term for the gaugino. In the non-abelian case, this result generalizes to

$$
\left.\frac{1}{8}\left(\mathcal{W}^{i \alpha} \mathcal{W}_{\alpha}^{i}\right)\right|_{F}+\text { h.c. }=\frac{1}{2}\left(D^{i}\right)^{2}+i \bar{\lambda}^{i} \bar{\sigma}^{\mu} D_{\mu} \lambda^{i}-\frac{1}{4} F_{\mu \nu}^{i} F^{i \mu \nu}
$$

Note that this Lagrangian contains self-interactions between gauge bosons as well as interactions between gauginos and gauge bosons. 


\subsubsection{Superpotential}

The last supersymmetric part of the Lagrangian to be included relies on the so-called superpotential. This is simply a holomorphic function of the chiral superfields that is a gauge singlet. Taking the $F$ part of the superpotential gives a Lagrangian that is supersymmetric and that codifies the interactions between chiral superfields. Given a superpotential $W$, the associated Lagrangian is given by

$$
\left.W\right|_{F}+\text { h.c. }
$$

Consider a set of superfields $\Phi_{i}$. The following identities are useful to extract a Lagrangian from the corresponding superpotential:

$$
\begin{aligned}
\left.\Phi_{i}\right|_{F} & =F_{i}, \\
\left.\Phi_{i} \Phi_{j}\right|_{F} & =\phi_{i} F_{j}+\phi_{j} F_{i}-\chi_{i} \cdot \chi_{j}, \\
\left.\Phi_{i} \Phi_{j} \Phi_{k}\right|_{F} & =\phi_{i} \phi_{j} F_{k}+\phi_{i} \phi_{k} F_{j}+\phi_{j} \phi_{k} F_{i}-\phi_{i} \chi_{j} \cdot \chi_{k}-\phi_{j} \chi_{i} \cdot \chi_{k}-\phi_{k} \chi_{i} \cdot \chi_{j} .
\end{aligned}
$$

\subsubsection{Potential}

As can easily be seen from the results of the last sections, the auxiliary fields do not possess any kinetic terms. It is therefore possible to use the equations of motion to remove them from the Lagrangian. Their removal leads to a potential for the scalar fields. Consider a superpotential $W$. Eliminating the $F$ fields leads to

$$
V_{F}=\sum_{i}\left|\frac{\partial W\left(\phi_{i}\right)}{\partial \phi_{i}}\right|^{2}
$$


where $W\left(\phi_{i}\right)$ means the superpotential with the chiral superfields replaced by their scalar component. In the case of an abelian gauge group, eliminating the field $D$ leads to

$$
V_{D}^{\text {Abelian }}=\sum_{i, j} \frac{g^{2}}{2}\left(\phi_{i}^{\dagger} Y_{i} \phi_{i}\right)\left(\phi_{j}^{\dagger} Y_{j} \phi_{j}\right)
$$

where $Y_{i}$ is the abelian charge of $\Phi_{i}$. In the non-abelian case, this gives

$$
V_{D}^{\text {Non-abelian }}=\sum_{a, i, j} \frac{g^{2}}{2}\left(\phi_{i}^{\dagger} \tau_{i}^{a} \phi_{i}\right)\left(\phi_{j}^{\dagger} \tau_{j}^{a} \phi_{j}\right)
$$

where $\tau_{i}^{a}$ is the representation of the algebra associated with $\phi_{i}$. Of course, there can also be contributions to the potential coming from soft SUSY breaking terms.

\subsection{The MSSM}

Having discussed all the necessary Supersymmetry background, it is now straightforward to find supersymmetric extensions of the Standard Model. The simplest possible one is the Minimal Supersymmetric Standard Model. The field content is summarized in Table 3.1. Every matter superfield is assumed left-handed chiral and every gauge superfield a vector superfield. Note that the Higgs content has been doubled. This has to do with the fact that having a single Higgs field provide masses to both types of quarks and charged leptons at the same time would only be possible via a non-holomorphic superpotential, i.e. the theory would not be supersymmetric. In

addition, the presence of a second Higgs partner is necessary for gauge anomalies to cancel. The scalar partners of fermions are denoted by adding a 's' before their name, e.g. the scalar partner of the top is the stop. Fermion partners are denoted by adding an 'ino' at the end of their name, e.g. the fermion partner of a Higgs boson is an Higgsino. The superpotential is given by 


\begin{tabular}{|c|c|c|c|}
\hline & $S U(3)_{C}$ & $S U(2)_{L}$ & $U(1)_{Y}$ \\
\hline$H_{u}$ & $\mathbf{1}$ & $\mathbf{2}$ & $1 / 2$ \\
\hline$H_{d}$ & $\mathbf{1}$ & $\mathbf{2}$ & $-1 / 2$ \\
\hline$Q^{m}$ & $\mathbf{3}$ & $\mathbf{2}$ & $1 / 6$ \\
\hline$U^{m}$ & $\overline{\mathbf{3}}$ & $\mathbf{1}$ & $-2 / 3$ \\
\hline$D^{m}$ & $\overline{\mathbf{3}}$ & $\mathbf{1}$ & $1 / 3$ \\
\hline$L^{m}$ & $\mathbf{1}$ & $\mathbf{2}$ & $-1 / 2$ \\
\hline$E^{m}$ & $\mathbf{1}$ & $\mathbf{1}$ & 1 \\
\hline$B_{\mu}$ & $\mathbf{1}$ & $\mathbf{1}$ & 0 \\
\hline$W_{\mu}^{a}$ & $\mathbf{1}$ & $\mathbf{3}$ & 0 \\
\hline$G_{\mu}^{b}$ & $\mathbf{8}$ & $\mathbf{1}$ & 0 \\
\hline
\end{tabular}

Table 3.1: Field content of the MSSM. The index $m$ goes from 1 to 3 , a from 1 to 3 and $b$ from 1 to 8 .

$$
W_{\mathrm{MSSM}}=y_{u}^{i j} U_{i} Q_{j} H_{u}-y_{d}^{i j} D_{i} Q_{j} H_{d}-y_{e}^{i j} E_{i} L_{j} H_{d}+\mu H_{u} H_{d}
$$

where $S U(2)$ indices are contracted antisymmetrically, e.g. $Q_{j} H_{u}=Q_{j}^{a}\left(i \sigma^{2}\right)_{a b} H_{u}^{b}$. In addition to this superpotential, it is also possible to write

$$
W_{\mathrm{RPV}}=\mu_{i} H_{u} L_{i}+\frac{\lambda_{i j k}}{2} L^{i} L^{j} E^{k}+\lambda_{i j k}^{\prime} L_{i} Q_{j} D_{k}+\frac{\lambda_{i j k}^{\prime \prime}}{2} U_{i} D_{j} D_{k} .
$$

This potential breaks baryon number and lepton number symmetries. It therefore leads to proton decay and many other strongly constrained scenarios [24]. These terms can be consistently set to zero by imposing what is known as $R$-parity. It is a $\mathbb{Z}_{2}$ symmetry under which the Grassmann variables are odd, while the Higgs superfields are even and the quarks and leptons superfields odd. It is easy to see that the terms of Eq. (3.34) are even while those of Eq. (3.35) are odd. Imposing $R$-parity then insures that the terms of Eq. (3.35) are not generated pertubatively. With the final step of imposing gauge superfields to be even under $R$-parity, the $R$-charge of a 
generic field can be written as

$$
R=(-1)^{3 B+L+2 s},
$$

where $B, L$ and $s$ are respectively the baryon number, lepton number and spin of a particle. This way, all Standard Model particles are even and their superpartners odd.

To complete the Lagrangian of the MSSM, it suffices to specify the soft SUSY breaking terms. The first contribution is the gaugino masses

$$
\mathcal{L}_{\text {gaugino masses }}^{\text {soft }}=-\frac{1}{2} M_{1} \tilde{B} \cdot \tilde{B}-\frac{1}{2} M_{2} \tilde{W}^{a} \cdot \tilde{W}^{a}-\frac{1}{2} M_{3} \tilde{G}^{b} \cdot \tilde{G}^{b}+\text { h.c. },
$$

where $\tilde{B}$ refers to the bino and so forth. The squarks and sleptons are also given soft masses

$$
\begin{aligned}
\mathcal{L}_{\text {sfermion masses }}^{\text {soft }}= & -\left(m_{Q}^{2}\right)_{i j} \tilde{Q}^{i \dagger} \tilde{Q}^{j}-\left(m_{U}^{2}\right)_{i j} \tilde{U}^{i \dagger} \tilde{U}^{j}-\left(m_{D}^{2}\right)_{i j} \tilde{D}^{i \dagger} \tilde{D}^{j} \\
& -\left(m_{L}^{2}\right)_{i j} \tilde{L}^{i \dagger} \tilde{L}^{j}-\left(m_{E}^{2}\right)_{i j} \tilde{E}^{i \dagger} \tilde{E}^{j},
\end{aligned}
$$

where $\tilde{U}$ is the scalar partner of a right-handed up quark and so forth. These terms are potentially very dangerous as they can give large contributions to flavour mixing processes that are strongly constrained. Mediation mechanisms must then be constructed such that this mixing is suppressed. The Higgs potential also contains soft terms. They are

$$
\mathcal{L}_{\text {Higgs potential }}^{\text {soft }}=-m_{H_{u}}^{2} H_{u}^{\dagger} H_{u}-m_{H_{d}}^{2} H_{d}^{\dagger} H_{d}-\left(B_{\mu} H_{u} H_{d}+\text { h.c. }\right),
$$

where we used the commonly used convention of referring to the scalar component 
of $H_{u}$ and $H_{d}$ by the same symbol. The soft terms are finally completed by a set of trilinear terms sometimes known as $A$-terms,

$$
\mathcal{L}_{A \text { terms }}^{\text {soft }}=-\left(\left(A_{u}\right)_{i j} \tilde{U}^{i} \tilde{Q}^{j} H_{u}-\left(A_{d}\right)_{i j} \tilde{D}^{i} \tilde{Q}^{j} H_{d}-\left(A_{e}\right)_{i j} \tilde{E}^{i} \tilde{L}^{j} H_{d}+\text { h.c. }\right) .
$$

\subsubsection{Electroweak symmetry breaking}

Electroweak symmetry breaking occurs once $H_{u}$ and $H_{d}$ acquire expectation values, which are denoted by $v_{u}$ and $v_{d}$ respectively. This leads to mixing that would have been forbidden had electroweak symmetry not been broken. The neutral Higgses mix like

$$
\left(\begin{array}{c}
H_{u}^{0} \\
H_{d}^{0}
\end{array}\right)=\left(\begin{array}{c}
v_{u} \\
v_{d}
\end{array}\right)+\frac{1}{\sqrt{2}} R_{\alpha}\left(\begin{array}{c}
h^{0} \\
H^{0}
\end{array}\right)+\frac{i}{\sqrt{2}} R_{\beta}\left(\begin{array}{c}
G^{0} \\
A^{0}
\end{array}\right) .
$$

The field $G^{0}$ is the Goldstone boson eaten by the $Z$ boson. The fields $h^{0}$ and $H^{0}$ are respectively light and heavy $C P$-even fields, while $A^{0}$ is $C P$-odd. The mixing matrices are given by

$$
R_{\alpha}=\left(\begin{array}{cc}
\cos \alpha & \sin \alpha \\
-\sin \alpha & \cos \alpha
\end{array}\right), \quad R_{\beta}=\left(\begin{array}{cc}
\sin \beta & \cos \beta \\
-\cos \beta & \sin \beta
\end{array}\right)
$$

where $\beta$ is defined by $\tan \beta=v_{u} / v_{d}$ and $\alpha$ can be found from the relations

$$
\frac{\sin 2 \alpha}{\sin 2 \beta}=-\left(\frac{m_{H^{0}}^{2}+m_{h^{0}}^{2}}{m_{H^{0}}^{2}-m_{h^{0}}^{2}}\right), \quad \frac{\tan 2 \alpha}{\tan 2 \beta}=\left(\frac{m_{A^{0}}^{2}+m_{Z}^{2}}{m_{A^{0}}^{2}-m_{Z}^{2}}\right)
$$


where the masses of the Higgses are given below. The charged Higgses mix as

$$
\left(\begin{array}{c}
H_{u}^{+} \\
H_{d}^{-*}
\end{array}\right)=R_{\beta}\left(\begin{array}{l}
G^{+} \\
H^{+}
\end{array}\right) .
$$

The field $G^{+}$is once again a Goldstone boson and is eaten by the $W$ boson. The masses of the physical Higgses are then given at tree-level by

$$
\begin{aligned}
m_{A^{0}}^{2} & =2|\mu|^{2}+m_{H_{u}}^{2}+m_{H_{d}}^{2}, \\
m_{h^{0}, H^{0}}^{2} & =\frac{1}{2}\left(m_{A^{0}}^{2}+m_{Z}^{2} \mp \sqrt{\left(m_{A^{0}}^{2}-m_{Z}^{2}\right)^{2}+4 m_{Z}^{2} m_{A^{0}}^{2} \sin ^{2} 2 \beta}\right), \\
m_{H^{+}} & =m_{A^{0}}^{2}+m_{W}^{2} .
\end{aligned}
$$

It is easy to see that at this stage $m_{h^{0}} \leq m_{Z}$. To accommodate the known Higgs mass of $125 \mathrm{GeV}$, it is then necessary for the Higgs mass to receive large radiative corrections.

In addition, electroweak symmetry breaking leads to mixing between neutralinos. In the $\psi^{0}=\left(\tilde{B}, \tilde{W}^{3}, \tilde{H}_{d}^{0}, \tilde{H}_{u}^{0}\right)$ basis, the mass matrix of neutralinos is defined by

$$
\mathcal{L}_{\text {Neutralino masses }}=-\frac{1}{2} \psi^{0 T} M_{\tilde{N}} \psi^{0}+\text { h.c. },
$$

where

$$
M_{\tilde{N}}=\left(\begin{array}{cccc}
M_{1} & 0 & -g^{\prime} v_{d} / \sqrt{2} & g^{\prime} v_{u} / \sqrt{2} \\
0 & M_{2} & g v_{d} / \sqrt{2} & -g v_{u} / \sqrt{2} \\
-g^{\prime} v_{d} / \sqrt{2} & g v_{d} / \sqrt{2} & 0 & -\mu \\
g^{\prime} v_{u} / \sqrt{2} & -g v_{u} / \sqrt{2} & -\mu & 0
\end{array}\right) .
$$

The MSSM then possesses four neutralinos. In the $\psi^{ \pm}=\left(\tilde{W}^{+}, \tilde{H}_{u}^{+}, \tilde{W}^{-}, \tilde{H}_{d}^{-}\right)$basis, 
the mixing between the charginos is encapsulated in

$$
\mathcal{L}_{\text {Chargino masses }}=-\frac{1}{2}\left(\psi^{ \pm}\right)^{T} M_{\tilde{C}} \psi^{ \pm}+\text {h.c. }
$$

where

$$
M_{\tilde{C}}=\left(\begin{array}{cc}
0 & X^{T} \\
X & 0
\end{array}\right)
$$

and

$$
X=\left(\begin{array}{cc}
M_{2} & g v_{u} \\
g v_{d} & \mu
\end{array}\right)
$$

The MSSM therefore possesses two charginos. Also of importance is that electroweak symmetry breaking leads to mixing between squarks. This is most important for the stops. In the $\phi_{\tilde{t}}=\left(\tilde{t}_{L}, \tilde{t}_{R}^{*}\right)$ basis, it is given by

$$
\mathcal{L}_{\text {Stop masses }}=-\phi_{\tilde{t}}^{\dagger} M_{\tilde{t}}^{2} \phi_{\tilde{t}}
$$

where

$$
M_{\tilde{t}}^{2}=\left(\begin{array}{cc}
m_{Q_{3}}^{2}+m_{t}^{2}+\Delta_{\tilde{u}_{L}} & v\left(A_{t}^{*} \sin \beta-\mu y_{t} \cos \beta\right) \\
v\left(A_{t} \sin \beta-\mu^{*} y_{t} \cos \beta\right) & m_{U_{3}}^{2}+m_{t}^{2}+\Delta_{\tilde{u}_{R}}
\end{array}\right),
$$

where $m_{t}$ is the mass of the top quark and $\Delta_{\tilde{u}_{L}}=\left(\frac{1}{2}-\frac{2}{3} \sin ^{2} \theta_{W}\right) \cos (2 \beta) m_{Z}^{2}$ and $\Delta_{\tilde{u}_{R}}=\left(\frac{2}{3} \sin ^{2} \theta_{W}\right) \cos (2 \beta) m_{Z}^{2}$ are radiative corrections. There are then two stops of different masses.

\subsection{Mediation mechanisms}

The soft masses that appear in the MSSM or other realistic supersymmetric models are assumed to come from a hidden sector in which Supersymmetry is broken. This 
is necessary because of the supertrace theorem, which states that for theories with no non-renormalizable interactions

$$
\mathrm{S} \operatorname{Tr} \mathcal{M}^{2}=\sum_{J}(-1)^{2 J}(2 J+1) \mathcal{M}_{J}^{2}=0
$$

where the sum is over all particles, $J$ is their spin and $\mathcal{M}_{J}$ is their tree-level mass. The case of tree-level SUSY breaking then generically involves dangerously light partners. It is therefore necessary to have a hidden sector that has no tree-level renormalizable interactions with the visible sector.

The transmission of SUSY breaking from the hidden sector to the low energy theory is known as mediation. We present three examples in this section. They are gravity mediation, gauge mediation [25-30] and anomaly mediation [31,32]. The latter two will play a major role in Chapter 5.

\subsubsection{Gravity mediation}

The idea behind gravity mediation is that the $F$ part of a hidden sector chiral superfield $X$ acquires an expectation value $\left\langle F_{X}\right\rangle$. SUSY breaking is then transmitted to the low energy theory via higher dimensional operators. The soft terms of Section 3.4 can then be obtained via a set of effective Lagrangians

$$
\begin{aligned}
& \mathcal{L}_{\text {gaugino masses }}^{\text {soft }}=-\int d^{2} \theta \frac{X}{4 M_{P l}}\left(\hat{M}_{1} \mathcal{W}_{1}^{\alpha} \mathcal{W}_{1 \alpha}+\hat{M}_{2} \mathcal{W}_{2}^{\alpha} \mathcal{W}_{2 \alpha}+\hat{M}_{3} \mathcal{W}_{3}^{\alpha} \mathcal{W}_{3 \alpha}\right)+\text { h.c. } \\
& \mathcal{L}_{\text {sfermion masses }}^{\text {soft }}=-\int d^{4} \theta \frac{X^{\dagger} X}{M_{P l}^{2}}\left(\left(\hat{m}_{Q}^{2}\right)_{i j} Q^{i \dagger} Q^{j}+\left(\hat{m}_{U}^{2}\right)_{i j} U^{i \dagger} U^{j}+\left(\hat{m}_{D}^{2}\right)_{i j} D^{i \dagger} D^{j}\right. \\
& \left.+\left(\hat{m}_{L}^{2}\right)_{i j} L^{i \dagger} L^{j}+\left(\hat{m}_{E}^{2}\right)_{i j} E^{i \dagger} E^{j}\right), \\
& \mathcal{L}_{\text {Higgs potential }}^{\text {soft }}=-\int d^{4} \theta \frac{X^{\dagger} X}{M_{P l}^{2}}\left(\hat{m}_{H_{u}}^{2} H_{u}^{\dagger} H_{u}+\hat{m}_{H_{d}}^{2} H_{d}^{\dagger} H_{d}\right)
\end{aligned}
$$




$$
\begin{aligned}
& -\int d^{4} \theta \frac{X^{\dagger} X}{M_{P l}^{2}} B_{\mu} H_{u} H_{d}+\text { h.c. } \\
\mathcal{L}_{A \text { terms }}^{\text {soft }}= & -\int d^{2} \theta \frac{X}{M_{P l}}\left(\left(\hat{A}_{u}\right)_{i j} U^{i} Q^{j} H_{u}-\left(\hat{A}_{d}\right)_{i j} D^{i} Q^{j} H_{d}-\left(\hat{A}_{e}\right)_{i j} E^{i} L^{j} H_{d}\right) \\
& + \text { h.c. }
\end{aligned}
$$

where we suppressed group indices, $M_{P l}$ is the Planck scale, $\mathcal{W}_{i}^{\alpha}$ are the gauge field superstrengths and all hatted parameters are dimensionless constants.

There exists a very famous special case of gravity mediation based on a series of assumptions. First, all scalars masses are set to a common value $m_{0}$ at the SUSY breaking scale and the gauginos masses are similarly set to $m_{1 / 2}$. The $A$-terms are assumed of the form $A_{f}=A y_{f}$, where $y_{f}$ is the corresponding Yukawa coupling and $A$ is a universal constant. The parameters $\mu$ and $B_{\mu}$ are used to set the known mass of the $Z$ boson and $\tan \beta$, which is taken as a free parameter. This procedure however does not set the sign of $\mu$. There are then four free parameters $\left(m_{0}, m_{1 / 2}, A, \tan \beta\right)$ and a sign $(\operatorname{sign}(\mu))$. Finally, all coefficients are evolved down from the unification scale to the electroweak scale via renormalization group equations. This scenario is known as minimal supergravity (MSUGRA) [33]. MSUGRA relaxes some of the constraints coming from flavour physics.

\subsubsection{Gauge mediation}

Gauge mediation relies on a messenger sector to transmit SUSY breaking from the hidden sector to the low energy theory. These messengers only interact with the low energy theory via gauge interactions. This results in flavour blind soft masses which alleviate the constraints from flavour physics. There are many variations and we give an example applicable to the MSSM. 
Consider a singlet chiral superfield $S$. Assume that its scalar component acquires an expectation value $\langle S\rangle$ and its $F$ part an expectation $\langle F\rangle$. We now introduce messenger fields that are charged under $S U(3)_{c} \times S U(2)_{L} \times U(1)_{Y}$ as

$$
q \sim\left(3,1,-\frac{1}{3}\right), \quad \bar{q} \sim\left(\overline{3}, 1, \frac{1}{3}\right), \quad l \sim\left(1,2, \frac{1}{2}\right), \quad \bar{l} \sim\left(1,2,-\frac{1}{2}\right)
$$

These interact with the singlet via the superpotential

$$
W_{\mathrm{mess}}=y_{2} S l \bar{l}+y_{1} S q \bar{q}
$$

A short calculation leads to the masses

$$
\begin{array}{ll}
l, \bar{l}: & m_{\text {fermions }}^{2}=\left|y_{2}\langle S\rangle\right|^{2}, \quad m_{\text {scalars }}^{2}=\left|y_{2}\langle S\rangle\right|^{2} \pm\left|y_{2}\langle F\rangle\right|, \\
q, \bar{q}: & m_{\text {fermions }}^{2}=\left|y_{1}\langle S\rangle\right|^{2}, \quad m_{\text {scalars }}^{2}=\left|y_{1}\langle S\rangle\right|^{2} \pm\left|y_{1}\langle F\rangle\right| .
\end{array}
$$

One loop diagrams involving the messengers lead to masses for the gauginos of

$$
M_{i}=\frac{g_{i}^{2}}{16 \pi^{2}} \frac{\langle F\rangle}{\langle S\rangle}
$$

where $i$ goes from 1 to $3, g_{1}=\sqrt{5 / 3} g^{\prime}$ is the GUT normalized $U(1)_{Y}$ gauge coupling constant and $g_{2}$ and $g_{3}$ are just $g$ and $g_{s}$ respectively.

The soft masses of the scalars and the $A$-terms will not be necessary for this work. We mention their expressions only for completeness sake. The soft mass of a scalar $\phi_{i}$ is given by

$$
m_{\phi_{i}}^{2}=2\left[\left(\frac{g_{1}}{16 \pi^{2}}\right)^{2} C_{1}(i)+\left(\frac{g_{2}}{16 \pi^{2}}\right)^{2} C_{2}(i)+\left(\frac{g_{3}}{16 \pi^{2}}\right)^{2} C_{3}(i)\right] \frac{\langle F\rangle^{2}}{\langle S\rangle^{2}}
$$

where the $C_{j}(i)$ are the different quadratic Casimir invariants for the representation 
under which $\phi_{i}$ transforms. The $A$-terms are generated at two loops and are very small.

\subsubsection{Anomaly mediation}

Anomaly mediation arises in the context of Supergravity (SUGRA) and can be seen as a consequence of the super-Weyl anomaly, which is the breaking of scale invariance by radiative corrections (i.e. the running of coupling constants). The most important SUGRA effects can be taken into account by the introduction of the so-called conformal compensator $\Phi$. Fixing it to a given value transforms a conformal SUGRA theory to a non-conformal one. In general, a field $A$ will transform under conformal transformations. By redefining $A \rightarrow \Phi^{p} A$ where $p$ is a real number, all the scale dependence can be contained in the conformal compensator. However, SUSY breaking in the hidden sector also provides an expectation value to the $F$ part of the conformal compensator, i.e. $\langle\Phi\rangle=1-m_{3 / 2} \theta^{2} / 2$. This is the origin of soft masses in anomaly mediation.

For example, consider the gaugino mass of a vector superfield $V$ with superfield strength $W^{\alpha}$ and associated gauge coupling constant $g$. To make things clearer, perform a field redefinition $\mathcal{W}^{\alpha} \rightarrow \mathcal{W}^{\alpha} / g$ such that the coupling constant appears only in the gaugino kinetic term. Running down the theory from the ultraviolet (UV) scale $\Lambda$ to a lower scale $\mu$ will lead to a renormalization of the kinetic term

$$
\left.\frac{1}{4} \frac{1}{g^{2}(\mu / \Phi \Lambda)} \mathcal{W}^{\alpha} \mathcal{W}_{\alpha}\right|_{F}+\text { h.c. }
$$

where the dependence of $g$ is imposed by classical conformal invariance. Taylor expanding $g$ in terms of the Grassmann variable and then canonically normalizing the 
fields leads to a gaugino mass of

$$
M=\frac{\beta}{g} m_{3 / 2},
$$

where

$$
\beta \equiv \frac{d g}{d \ln \mu}
$$

For the MSSM, this gives

$$
M_{1}=\frac{33 g_{1}^{2}}{80 \pi^{2}} m_{3 / 2}, \quad M_{2}=\frac{g_{2}^{2}}{16 \pi^{2}} m_{3 / 2}, \quad M_{3}=-\frac{3 g_{3}^{2}}{16 \pi^{2}} m_{3 / 2}
$$

where the negative sign of $M_{3}$ can be reabsorbed by a field redefinition. The soft mass of a scalar $\phi_{i}$ is given by a similar expression,

$$
m^{2}=-\frac{1}{4}\left|m_{3 / 2}\right|^{2} \frac{d \gamma}{d \ln \mu},
$$

where $\gamma$ is the anomalous dimension of $\phi_{i}$

$$
\gamma=\frac{d \ln Z}{d \ln \mu},
$$

where $Z$ is the field-strength renormalization of $\phi_{i}$. Finally, the $A$-terms are given by

$$
A^{i j k}=-m_{3 / 2} \beta_{y^{i j k}},
$$

where $\beta_{y^{i j k}}$ is the beta function of the corresponding Yukawa. 


\section{Chapter 4}

\section{The spontaneous $\mathbb{Z}_{2}$ breaking Twin}

\section{Higgs}

Many beyond the Standard Model (BSM) theories seek to stabilize the electroweak scale by introducing partners to some particles of the Standard Model. Supersymmetry is the most famous example of this. However, these new particles are generally charged under the Standard Model gauge groups and naturalness requires that at least the partners of the SM particles that contribute the most to the Higgs mass be close to the electroweak scale. It was therefore expected that these partners would be abundantly produced at previous or current collider experiments. The lack of discovery of such particles imposes severe constraints on their corresponding theories and in turn leads to some tension concerning naturalness.

An alternative approach is to have the stabilizing partners be charged under gauge groups that differ (at least partially) from those of the Standard Model. This can result in the partners communicating very little with the Standard Model sector, more often than not only via the Higgs, which makes their production at colliders more difficult. This therefore relaxes most experimental constraints and naturalness can 
then potentially be restored. This concept goes under the name of neutral naturalness.

The two seminal examples of neutral naturalness are Folded Supersymmetry [34] and Twin Higgs [35] (see also [36-53]). This chapter mainly focuses on the latter model, but Folded Supersymmetry is summarized here for completeness sake. The simplest realization of Folded Supersymmetry aims to cancel the largest correction to the Higgs mass, which comes from top loops. As in Supersymmetry, this correction can be canceled by introducing scalar tops called stops. Folded Supersymmetry goes further by expanding the gauge groups to $S U(3)_{F} \times S U(3)_{c} \times S U(2)_{L} \times U(1)_{Y}$. Fermionic top partners are introduced that are charged under $S U(2)_{L}$ and $U(1)_{Y}$ as is the top, but that are charged under $S U(3)_{F}$ instead of $S U(3)_{c}$. These top partners, referred to as folded-tops, reintroduce large corrections to the Higgs mass. These corrections are canceled in turn by introducing scalar partners to the folded-top, which are referred to as folded-stops. Overall, the cancellation of the quadratic corrections is between the top and stops and between the folded-top and folded-stops. If a $\mathbb{Z}_{2}$ symmetry is imposed between the $S U(3)_{c}$ and $S U(3)_{F}$ groups, the contribution from the top and folded-top are numerically the same, as are those from the stops and folded-stops. The cancellation can then be done between the top and folded-stops. There is no need anymore for the stops and folded-top. They can therefore be assumed heavy and integrated out. The end result is that the top partners that stabilize the electroweak scale are not charged under $S U(3)_{c}$. Their cross sections at hadron colliders are therefore strongly decreased and experimental constraints are vastly reduced (see Ref. [51] for a few examples). Such a particle spectrum can result from a supersymmetric model with an extra compactified dimension using Scherk-Schwartz Supersymmetry breaking $[54,55]$.

On the other hand, the Twin Higgs rests on a global $S U(4)$ which is broken 
spontaneously to $S U(3)$ at a scale $f$, leading to a set of Goldstone bosons. The $S U(4)$ is explicitly broken by gauging a $S U(2)_{A} \times S U(2)_{B}$ subgroup (with $S U(2)_{A}$ being identified with the SM $S U(2)_{L}$ and $S U(2)_{B}$ a similar symmetry of a mirror sector) and by adding Yukawa couplings. In principle, this breaking would give a mass of order $f$ to the Goldstone bosons. Remarkably, imposing a $\mathbb{Z}_{2}$ symmetry between the two sectors ensures that the theory is still $S U(4)$ invariant at the quadratic level, leading to a light pseudo-Goldstone Higgs. A soft $\mathbb{Z}_{2}$ breaking is however needed to obtain a hierarchy of vacuum expectation values between the Standard Model Higgs and the mirror sector Higgs $[35,36]^{1}$

Despite its success, even the Twin Higgs is not free from tuning. A moderate amount of tuning between the $\mathbb{Z}_{2}$ and the $S U(4)$ breaking sectors is needed to push the cutoff beyond experimental constraints and be compatible with the Higgs signal strengths measurements. Various attempts at addressing this issue can be found in the literature. Ref. [56] tries to do so in the context of a two Higgs doublet model with misaligned vevs. In Ref. [57], the issue is addressed in a supersymmetric UV completion by introducing Dirac gauginos [58]. Finally, Ref. [59] also addresses the supersymmetric completion, but by forcing $\tan \beta=1$ in the mirror sector. Both of these models try to remove the D-term quartics which are a source of tuning in supersymmetric versions of the Twin Higgs. One thing all of these models have in common is an explicit $\mathbb{Z}_{2}$ breaking.

This chapter presents a novel approach to improving the tuning in Twin Higgs, which is based on spontaneous breaking of the $\mathbb{Z}_{2}$ symmetry. The proposed model, referred to from now on as the spontaneous $\mathbb{Z}_{2}$ breaking Twin Higgs, includes two Higgses in the fundamental representation of a $S U(4)$ global symmetry. As in the original Twin Higgs model, $S U(4)$ is broken by gauging a $S U(2)_{A} \times S U(2)_{B}$ subgroup

\footnotetext{
${ }^{1}$ See section 4.1 .1 for more details.
} 
and by introducing Yukawa interactions. $\mathrm{A} \mathbb{Z}_{2}$ symmetry is then imposed between the two sectors. We take the vacuum of the first Higgs to preserve $\mathbb{Z}_{2}$, while the other breaks it spontaneously. A bilinear term containing the two Higgses is added (similar to the $B_{\mu}$ term of the MSSM) and the $\mathbb{Z}_{2}$ breaking is transmitted from the broken to the unbroken sector. This naturally produces a hierarchy between the vevs of the SM sector Higgses and those of the mirror sector. The $B_{\mu}$ term acts as an effective tadpole and no explicit $\mathbb{Z}_{2}$ breaking is necessary. The presence of this effective tadpole and feedback between the two Higgses combine to reduce the tuning compared to the original Twin Higgs as the resulting Higgs boson is naturally very SM-like.

The chapter is organized as follows. We begin by summarizing the original Twin Higgs model to isolate the origin of the tuning and obtain results that will make comparisons with our model easier. The spontaneous $\mathbb{Z}_{2}$ breaking Twin Higgs is then presented in detail. An analysis of the radiative corrections follows. A detailed analysis of the tuning of the model compared to the original Twin Higgs is then performed. Finally, a few concluding remarks about possible UV completions are presented.

\subsection{The model}

\subsubsection{The original Twin Higgs}

To put in context the problem the spontaneous $\mathbb{Z}_{2}$ breaking Twin Higgs attempts to solve and to establish the notation used in this chapter, we dedicate this section to a review of the Twin Higgs. We follow closely Ref. [35], albeit with modified notation.

Assume a complex scalar field $H$ which is a fundamental of a global $S U(4)$. Its 
symmetry preserving potential can be written as

$$
V_{S U(4)}(H)=-\mu^{2} H^{\dagger} H+\lambda\left(H^{\dagger} H\right)^{2}
$$

Positive values of $\mu^{2}$ cause $S U(4)$ to be broken spontaneously to $S U(3)$. This leads to $\langle H\rangle \equiv f=\mu / \sqrt{2 \lambda}$ and 7 Goldstone bosons. The goal is to associate the Standard Model Higgs with one of these Goldstone bosons, which are still at this point massless. Making such a Goldstone a viable Higgs boson however requires to explicitly break $S U(4)$.

The $S U(4)$ symmetry is first explicitly broken by gauging one of its $S U(2)_{A} \times$ $S U(2)_{B}$ subgroups. The field $H$ can then be divided into fundamentals of $S U(2)_{A}$ and $S U(2)_{B}$ as $H=\left(H_{A}, H_{B}\right)$. The $A$ sector is conventionally associated to the Standard Model and the $B$ sector to the mirror sector. This gauging of a subgroup of $S U(4)$ will be reflected in the potential via radiative corrections. The leading corrections to the potential introduced by this gauging are

$$
\Delta V(H)=\frac{9 g_{A}^{2} \Lambda^{2}}{64 \pi^{2}} H_{A}^{\dagger} H_{A}+\frac{9 g_{B}^{2} \Lambda^{2}}{64 \pi^{2}} H_{B}^{\dagger} H_{B}
$$

where $g_{A}$ and $g_{B}$ are the gauge coupling constants of $S U(2)_{A}$ and $S U(2)_{B}$ respectively and $\Lambda$ is the cutoff of the theory. If a $\mathbb{Z}_{2}$ symmetry is imposed between the $A$ and $B$ sectors, the coefficients of the two terms of Eq. (4.2) become the same. These corrections can then be rewritten as a term of the form $H^{\dagger} H$, which respects the original $S U(4)$ symmetry. The Goldstone bosons therefore do not acquire any mass from Eq. (4.2) in the presence of a $\mathbb{Z}_{2}$ symmetry. However, the subleading corrections $\kappa\left(\left|H_{A}\right|^{4}+\left|H_{B}\right|^{4}\right)$, where $\kappa$ is of order $g^{2} / 16 \pi^{2} \ln (\Lambda / f)$, will explicitly break $S U(4)$. These logarithmic divergences can be reabsorbed in $\lambda$ and a $S U(4)$ breaking potential 
of the form

$$
V_{S U(4)}(H)=\alpha H_{A}^{\dagger} H_{A} H_{B}^{\dagger} H_{B} .
$$

A similar story holds for the top Yukawa coupling. A $\mathbb{Z}_{2}$ symmetry is imposed on this sector by adding a 'mirror top' which is not charged under the SM groups, but which couples to $H_{B}$ in exactly the same way in which the SM top couples to $H_{A}$.

The total potential at this point is the sum of Eqs. (4.1) and (4.3). The end result is that, of the original 7 Goldstone bosons, 6 remain massless and are eaten by massive gauge bosons and the one left over is a light pseudo-Goldstone boson that can be identified with the $125 \mathrm{GeV}$ Higgs. Since $\alpha$ is the only term in the potential that breaks $S U(4)$, it can naturally be smaller than $\lambda$, which is assumed from now on. This insures that the Higgs remains light even for relatively large $f$.

The symmetry breaking structure is controlled by the sign of $\alpha$ [36]. If $\alpha<0$, the minimum preserves $\mathbb{Z}_{2}$ and $\left\langle H_{A}\right\rangle=\left\langle H_{B}\right\rangle=\mu / \sqrt{4 \lambda+\alpha} \approx 174 \mathrm{GeV}$. This is the sign of $\alpha$ assumed in the original Twin Higgs model. The fact that $\left\langle H_{A}\right\rangle=\left\langle H_{B}\right\rangle$ leads to the Standard Model Higgs strongly mixing with the mirror sector Higgs and results in large deviations of the Higgs measurements [36]. It also means that $f$ is only slightly above the electroweak scale. The energy scale $\sim 4 \pi f$, at which new physics needs to appear to avoid fine-tuning, is then not much larger than in the Standard Model. These issues are easily resolved by aligning the vev closer to the $B$ sector, thereby allowing for a larger $f$ while preserving $\left\langle H_{A}\right\rangle=174 \mathrm{GeV}$. This can be done via an explicit soft $\mathbb{Z}_{2}$ breaking potential of the form

$$
V_{\mathbb{Z} / 2}(H)=\Delta m^{2} H_{A}^{\dagger} H_{A} .
$$

It is called soft as it does not reintroduce quadratic divergences in the Higgs mass, as 
is easily checked. The parameter $\Delta m^{2}$ can naturally be small as it is the only term that explicitly breaks $\mathbb{Z}_{2}$. The potential can be minimized by using the following parametrization of the relevant parts of $H$,

$$
H=f\left(\begin{array}{c}
0 \\
\sin \theta \\
0 \\
\cos \theta
\end{array}\right) \text {, }
$$

with $\theta$ being $\pi / 4$ when $\Delta m^{2}$ is 0 . The potential is minimized for a value of $f$ of

$$
f^{2}=\frac{2 \mu^{2}-\Delta m^{2}}{4 \lambda+\alpha}
$$

while minimizing the potential with respect to $\theta$ gives the following equation

$$
\alpha f^{4} \sin 4 \theta+4 \Delta m^{2} f^{2} \sin \theta \cos \theta=0 .
$$

This equation only yields a non-zero $\theta$ for $\Delta m^{2}$ below a maximal value. Thus, we can define $\Delta m_{\max }^{2}$ as the largest value of $\Delta m^{2}$ for which there is still electroweak symmetry breaking in the $A$ sector. It can be found by rewriting Eq. (4.7) as

$$
F_{1}(\theta) \equiv \frac{1}{4} \frac{\sin 4 \theta}{\sin \theta \cos \theta}=\frac{\Delta m^{2}}{\left(-\alpha f^{2}\right)} \approx \frac{\Delta m^{2}}{\Delta m_{\max }^{2}}
$$

where the last relation holds in the limit of small $\alpha$ and $\Delta m_{\max }^{2}$ is given by the exact relation

$$
\Delta m_{\max }^{2}=-\frac{\alpha \mu^{2}}{2 \lambda}
$$


The solution to Eq. (4.7) is

$$
\sin ^{2} \theta=\frac{v^{2}}{f^{2}}=\frac{1}{2}\left(1-\frac{\Delta m^{2}}{\left(-\alpha f^{2}\right)}\right) \approx \frac{1}{2}\left(1-\frac{\Delta m^{2}}{\Delta m_{\max }^{2}}\right),
$$

where $v$ is the SM Higgs vev of $174 \mathrm{GeV} .{ }^{2}$ Requesting a large $f$ implies a tuning between the $S U(4)$ breaking and the $\mathbb{Z}_{2}$ breaking potentials. This is reflected in Eq. (4.10) by the last term on the right needing to be close to 1 .

Alternatively, one can take $\alpha>0$. The $\mathbb{Z}_{2}$ symmetry is then spontaneously broken and the system falls in one of the two minima at $\left\langle H_{A}\right\rangle=\mu / \sqrt{2 \lambda}$ and $\left\langle H_{B}\right\rangle=0$ or $\left\langle H_{A}\right\rangle=0$ and $\left\langle H_{B}\right\rangle=\mu / \sqrt{2 \lambda}$. However, the vev must be taken to fall in the SM sector and this leads to a massless mirror sector. This proves to be unviable cosmologically as its leads to too much radiation during Big Bang Nucleosynthesis and less dark matter energy density than that of baryons [36]. It would also be disastrous for Higgs coupling strengths. The potential must then be modified in a way similar to Eq. (4.4) to prevent the minimum from being in one sector only. Unfortunately, a quick inspection shows that no term that only breaks $\mathbb{Z}_{2}$ softly and respects gauge invariance can do so. The term of Eq. (4.4) does not solve this problem, as Eq. (4.7) is satisfied by a $\theta$ of 0 for all values of $\Delta m^{2}$. The case of $\alpha>0$ therefore poses serious issues.

\subsubsection{Spontaneous $\mathbb{Z}_{2}$ breaking}

In the last section, part of the problem in the $\alpha>0$ case was that $H$ was the only scalar with gauge charges. This forced all terms in the potential to be an even power of $H$ and forbade tadpole terms, which could have potentially prevented the vev from

\footnotetext{
${ }^{2}$ In this chapter, we use a convention for $v$ that differs from the rest of the thesis by a factor of $\sqrt{2}$. This is simply for convenience sake and does not cause any ambiguities.
} 
falling in one sector only. The inclusion of a second Higgs field can solve this problem by including a term linear in both fields which acts as an effective tadpole for $H$ (see Ref. [60] for a similar idea in a context unrelated to $\mathbb{Z}_{2}$ breaking or the Twin Higgs). In addition, the $\mathbb{Z}_{2}$ breaking soft term also needed to be quadratic in $H$ for $\alpha<0$. It is possible that a similar term with a lower power of $H$ could potentially produce the same hierarchy of vevs while requiring less tuning. Again, a term linear in $H$ and another Higgs can do this.

Taking these considerations into account, the Higgs sector is extended to two fundamentals of $S U(4), H_{1}=\left(H_{1 A}, H_{1 B}\right)$ and $H_{2}=\left(H_{2 A}, H_{2 B}\right)$, that are gauged as in Twin Higgs and which interact with each other to create a hierarchy of vevs. The minimum of $H_{1}$ is taken to preserve $\mathbb{Z}_{2}$ and that of $H_{2}$ to break it. It is the interaction between $H_{1}$ and $H_{2}$ that transmits the $\mathbb{Z}_{2}$ breaking to $H_{1}$ and there is no need for an explicit $\mathbb{Z}_{2}$ breaking. This model is referred to as the spontaneous $\mathbb{Z}_{2}$ breaking Twin Higgs. The finer details are explained below.

We note that doubling the number of Higgses is a necessary element of a supersymmetric UV completion of the Twin Higgs. As such, adding a second Higgs does not really constitute an artificial extension of the model.

\section{Potential and vevs}

As a starting point, the potential for $H_{1}$ by itself is given by

$$
V_{H_{1}}\left(H_{1}\right)=-\mu_{1}^{2} H_{1}^{\dagger} H_{1}+\lambda_{1}\left(H_{1}^{\dagger} H_{1}\right)^{2}+\alpha_{1} H_{1 A}^{\dagger} H_{1 A} H_{1 B}^{\dagger} H_{1 B}
$$

and $\alpha_{1}$ is assumed negative, which means that the vacuum preserves $\mathbb{Z}_{2}$. At this point, the pseudo-Goldstone boson from $H_{1}$ corresponds to the angular mode and is an equal admixture of the components of $H_{1 A}$ and $H_{1 B}$. Similarly, the potential for 
$H_{2}$ by itself is

$$
V_{H_{2}}\left(H_{2}\right)=-\mu_{2}^{2} H_{2}^{\dagger} H_{2}+\lambda_{2}\left(H_{2}^{\dagger} H_{2}\right)^{2}+\alpha_{2} H_{2 A}^{\dagger} H_{2 A} H_{2 B}^{\dagger} H_{2 B}
$$

and this time with $\alpha_{2}>0$, meaning that the vacuum breaks $\mathbb{Z}_{2}$ in this case. The vev is taken to fall in the $B$ sector by convention, as the vev falling in the other sector would just mean a relabelling of $B$ as the SM and $A$ as the mirror sector. The pseudo-Goldstone boson again corresponds to the angular mode. This time however, the position of the minimum means that the pseudo-Goldstone boson is purely a component of $\mathrm{H}_{2 A}$.

The interaction between these two fields is then codified by the following Lagrangian

$$
V_{H_{1} H_{2}}\left(H_{1}, H_{2}\right)=-B_{\mu} H_{1}^{\dagger} H_{2}+\text { h.c. }
$$

It is technically natural to have $B_{\mu}$ small as it breaks a Peccei-Quinn symmetry. For $B_{\mu}$ small and greater than zero, Eq. (4.13) serves essentially two purposes. First, the part $H_{1 B}^{\dagger} H_{2 B}$ serves as an effective tadpole for $H_{1 B}$. It therefore pushes the vev of $H_{1}$ toward the $B$ sector, as desired. Second, the part $H_{1 A}^{\dagger} H_{2 A}$ serves as an effective tadpole for $H_{2 A}$. It accordingly provides a small positive $A$ component to the vev of $H_{2}$. As $B_{\mu}$ increases, non-linear effects and feedback between the different terms become important. An example of the different vevs is shown in Fig. 4.1.

\section{Small $\alpha_{i}$ 's approximation}

To gain a better understanding of the interactions between $H_{1}$ and $H_{2}$, we decompose them in a similar way to Eq. (4.5) and take the limit of small $\alpha_{i}$ 's. As will be made clear in Eq. (4.19), $B_{\mu}$ will be a factor of $\alpha_{1} / \lambda_{1}$ smaller than the $\mu_{i}^{2}$ 's in the physically 


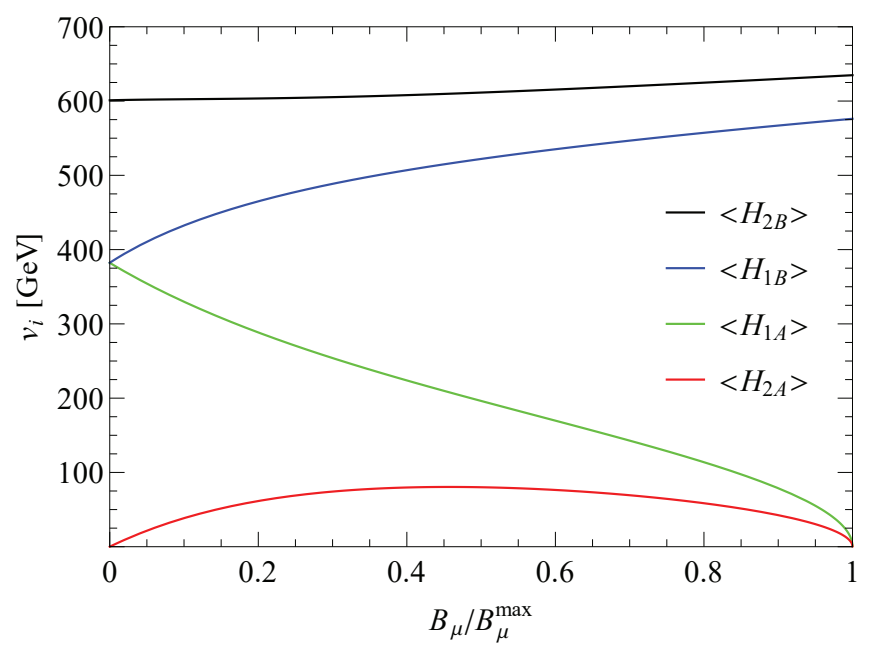

Figure 4.1: Example of the different vevs as a function of $B_{\mu} / B_{\mu}^{\max }$. The parameters are $\mu_{1}=750 \mathrm{GeV}, \mu_{2}=850 \mathrm{GeV}, \alpha_{1}=-0.15, \alpha_{2}=0.2$ and $\lambda_{1}=\lambda_{2}=1$.

viable and natural region of parameter space. It is therefore assumed to be small. In general, all approximations will be valid up to $\mathcal{O}\left(\alpha_{i} / \lambda_{i}\right)$. The decomposition of the Higgses is

$$
\left\langle H_{1}\right\rangle=f_{1}\left(\begin{array}{c}
0 \\
\sin \theta_{1} \\
0 \\
\cos \theta_{1}
\end{array}\right), \quad\left\langle H_{2}\right\rangle=f_{2}\left(\begin{array}{c}
0 \\
\sin \theta_{2} \\
0 \\
\cos \theta_{2}
\end{array}\right),
$$

where $f_{1} \approx \mu_{1} / \sqrt{2 \lambda_{1}}$ and $f_{2} \approx \mu_{2} / \sqrt{2 \lambda_{2}}$. The minimization of the potential with respect to the angles leads to the set of equations

$$
\begin{aligned}
& \alpha_{1} f_{1}^{4} \sin 4 \theta_{1}+4 B_{\mu} f_{1} f_{2} \sin \left(\theta_{1}-\theta_{2}\right)=0, \\
& \alpha_{2} f_{2}^{4} \sin 4 \theta_{2}-4 B_{\mu} f_{1} f_{2} \sin \left(\theta_{1}-\theta_{2}\right)=0 .
\end{aligned}
$$


When $B_{\mu}=0$, the minimum is located at $\theta_{1}=\pi / 4$ and $\theta_{2}=0$. In the general case, adding both equations leads to

$$
\sin 4 \theta_{2}=\Omega \sin 4 \theta_{1}
$$

where $\Omega$ is a constant in the small $\alpha_{i}$ 's approximation and is defined by

$$
\Omega \equiv-\frac{\alpha_{1}}{\alpha_{2}}\left(\frac{f_{1}}{f_{2}}\right)^{4}
$$

Eq. (4.16) is particularly interesting as it makes it clear that the exact details of the $\mathbb{Z}_{2}$ symmetry breaking depend on whether $\Omega$ is smaller or bigger than 1 . First, consider $\Omega<1$. Increasing $B_{\mu}$ will make $\theta_{1}$ pass from $\pi / 4$ to 0 . The angle $\theta_{2}$ starts by increasing but decreases once $\theta_{1}$ drops below $\pi / 8$. Eventually, both angles settle at 0 . When $\Omega>1$, this behavior is reversed. Increasing $B_{\mu}$ will make $\theta_{2}$ pass from 0 to $\pi / 4$. The angle $\theta_{1}$ decreases until $\theta_{2}$ reaches $\pi / 8$, but increases afterward. Both angles ultimately settle to $\pi / 4$ and the $\mathbb{Z}_{2}$ symmetry is restored. This behavior is not bad in itself as it can still lead to a small hierarchy, but obtaining a large one proves to be difficult. Taking these considerations into account, we focus on the domain where $\Omega<1$.

In analogy to the Twin Higgs case, we define $B_{\mu}^{\max }$ as the largest value of $B_{\mu}$ for which there is still electroweak symmetry breaking in the $A$ sector. The first line of Eq. (4.15) can then be rewritten as

$$
F_{2}\left(\theta_{1}, \Omega\right) \equiv \frac{(1-\Omega)}{4} \frac{\sin 4 \theta_{1}}{\sin \left(\theta_{1}-\theta_{2}\right)}=\frac{B_{\mu}}{\left(-\frac{\alpha_{1} f_{1}^{3}}{f_{2}(1-\Omega)}\right)} \approx \frac{B_{\mu}}{B_{\mu}^{\max }}
$$




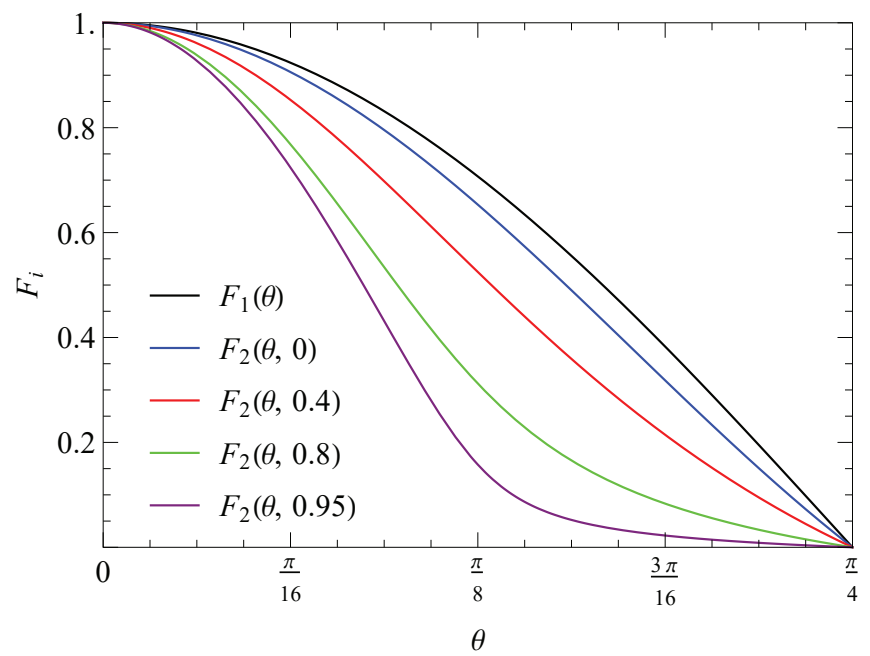

Figure 4.2: $F_{1}(\theta)$ and $F_{2}(\theta, \Omega)$ for different values of $\Omega$.

where $\theta_{2}$ is related to $\theta_{1}$ by Eq. (4.16). In the small $\alpha_{i}$ 's approximation, $B_{\mu}^{\max }$ is then

$$
B_{\mu}^{\max } \approx-\frac{\alpha_{1} f_{1}^{3}}{f_{2}(1-\Omega)}
$$

Note that $B_{\mu}^{\max }$ goes to infinity as $\Omega$ goes to 1 , which just reflects the fact that $B_{\mu}^{\max }$ doesn't exist for $\Omega>1$. While it is hard to solve Eq. (4.18) for $\theta_{1}$, it is easy to see that small values of $\theta_{1}$ require $B_{\mu}$ to be close to $B_{\mu}^{\max }$. This is similar to the Twin Higgs case where $\Delta m^{2}$ needed to be close to $\Delta m_{\max }^{2}$ to obtain a small ratio of vevs.

The two theories can be compared by looking at $F_{1}(\theta)$ versus $F_{2}(\theta, \Omega)$ which are plotted in Fig. 4.2 for different values of $\Omega$ between 0 and 1 . When $0<\theta<\pi / 4$, $F_{2}(\theta, \Omega)$ is always smaller than $F_{1}(\theta)$. This means that, for the same angle, the spontaneous $\mathbb{Z}_{2}$ breaking Twin Higgs doesn't require $B_{\mu}$ as close to $B_{\mu}^{\max }$ as the Twin Higgs requires $\Delta m^{2}$ close to $\Delta m_{\max }^{2}$. This translates to less tuning. In contrast to the Twin Higgs, one must keep in mind that for the proposed model $\left\langle H_{1 A}\right\rangle<v=174$ $\mathrm{GeV}$, as it is a two Higgs doublet model. As avoiding large tuning requires new physics near $\sim 4 \pi f_{1}$, this suggests that for equivalent tuning and cutoff one must choose $\theta_{1}$ 
smaller than the equivalent angle in Twin Higgs. Fortunately, the spontaneous $\mathbb{Z}_{2}$ breaking Twin Higgs naturally leads to $\left\langle H_{1 A}\right\rangle$ considerably larger than $\left\langle H_{2 A}\right\rangle$. Thus, the difference is small and the argument about tuning remains valid.

Further insight can be obtained by solving Eq. (4.18) in the small $\theta_{1}$ limit

$$
\theta_{1}^{2} \approx \frac{3}{8} \frac{\left(B_{\mu}^{\max }-B_{\mu}\right)}{\left(B_{\mu}^{\max }+g(\Omega) B_{\mu}\right)} \stackrel{B_{\mu} \rightarrow B_{\mu}^{\max }}{\sim} \frac{3}{8(1+g(\Omega))}\left(1-\frac{B_{\mu}}{B_{\mu}^{\max }}\right)
$$

where

$$
g(\Omega) \equiv \frac{1}{16}\left(15 \Omega^{2}+18 \Omega-1\right)
$$

As mentioned above, a more appropriate quantity to make the comparison with the Twin Higgs is

$$
\begin{aligned}
\frac{v^{2}}{f_{1}^{2}} & \sim \frac{3}{8(1+g(\Omega))}\left(1+\left(-\frac{\alpha_{2}}{\alpha_{1}}\right)^{-1 / 2} \Omega^{3 / 2}\right)\left(1-\frac{B_{\mu}}{B_{\mu}^{\max }}\right) \\
& \equiv C\left(-\alpha_{2} / \alpha_{1}, \Omega\right)\left(1-\frac{B_{\mu}}{B_{\mu}^{\max }}\right) .
\end{aligned}
$$

This is to be compared to Eq. (4.10) which has a similar structure but with the parameter $C\left(-\alpha_{2} / \alpha_{1}, \Omega\right)$ replaced by $1 / 2$. Fig. 4.3 shows $C\left(-\alpha_{2} / \alpha_{1}, \Omega\right)$ as a function of $\Omega$ for fixed values of $-\alpha_{2} / \alpha_{1}$. We see that, unless $-\alpha_{2} / \alpha_{1}$ is very small, $C\left(-\alpha_{2} / \alpha_{1}, \Omega\right)$ is smaller than $1 / 2$ for $\Omega$ in the whole range of 0 to 1 . This shows that the spontaneous $\mathbb{Z}_{2}$ breaking Twin Higgs can easily obtain the same cutoff as the Twin Higgs for less tuning.

The improvement in the tuning can ultimately be attributed to two sources. First, we can look at the limit of small $\Omega$, which means that $\theta_{2}$ is also small. This limit means that $H_{2}$ only serves as an effective tadpole and does not mix with $H_{1}$. The fact that the symmetry breaking is induced by an effective tadpole translates to 


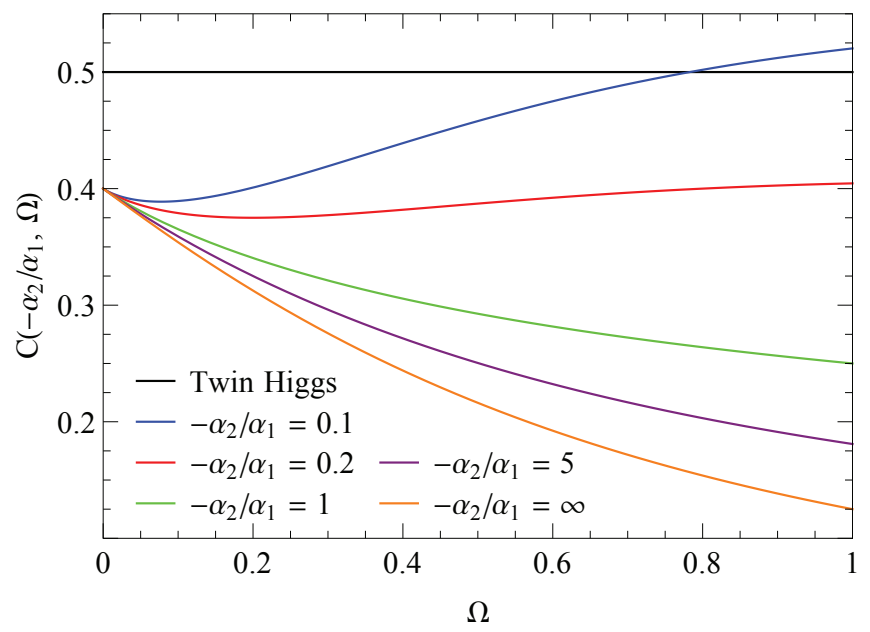

Figure 4.3: $C\left(-\alpha_{2} / \alpha_{1}, \Omega\right)$ for various values for $-\alpha_{2} / \alpha_{1}$. Also shown is the corresponding value for Twin Higgs.

Eq. (4.18) missing the factor of $\cos \theta$ present in Eq. (4.8). This by itself is enough to insure that $F_{2}(\theta, \Omega)$ be smaller than $F_{1}(\theta)$. Second, there is considerable feedback between $H_{1}$ and $H_{2}$ when $\Omega$ is close to 1 . This translates to $\theta_{2}$ and $1-\Omega$ appearing in Eq. (4.18). The presence of these terms further decreases $F_{2}(\theta, \Omega)$, as is clearly shown in Fig. 4.2. Obviously, taking $\Omega$ close to 1 is a tuning in itself, though certainly not large enough to spoil our results, and we take this into account in Section 4.2.

\section{Additional properties}

A few additional properties of the model are worth mentioning.

Existence of $B_{\mu}^{\max }$. The first property to mention is that the behavior of Fig. 4.1 can differ outside of the region of parameter space considered up to now. The case of $\Omega>1$ mentioned above is an example. An example of the behavior of the vevs in this case is shown in Fig. 4.4. Even when $\Omega<1$, the vevs can act differently if the $\alpha_{i}$ 's or $B_{\mu}$ are large. In particular, it is possible to choose parameters such that the vevs of the $A$ sector start like those of Fig. 4.1 but fail to reach 0 . It is also possible 


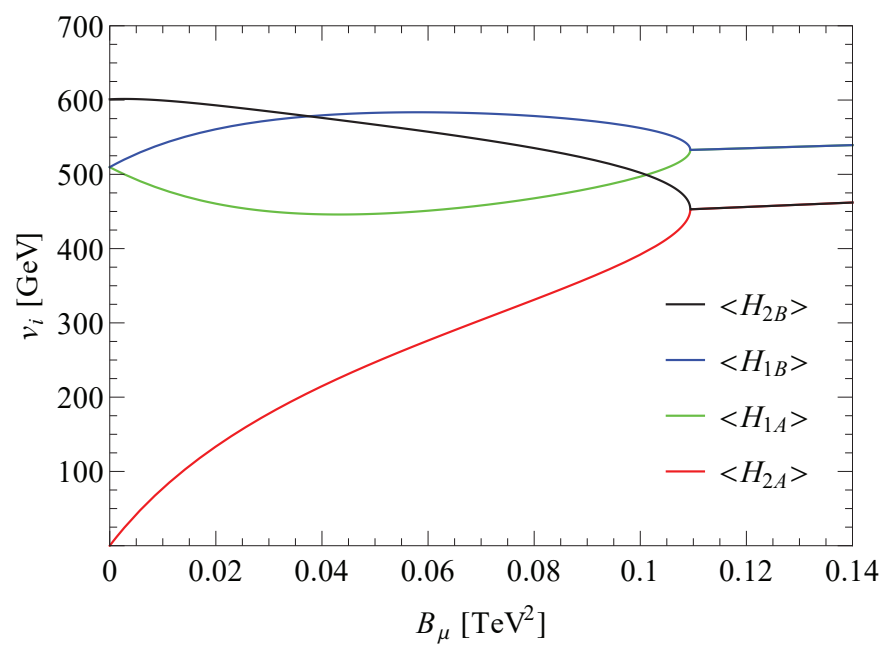

Figure 4.4: Example of the different vevs as a function of $B_{\mu}$ for $\Omega>1$. The parameters are $\mu_{1}=1000 \mathrm{GeV}, \mu_{2}=850 \mathrm{GeV}, \alpha_{1}=-0.15, \alpha_{2}=0.15$ and $\lambda_{1}=\lambda_{2}=$ 1.

for the vevs of the $A$ sector to be 0 for an interval of $B_{\mu}$ but then become non-zero again for very large $B_{\mu}$. The parameter $B_{\mu}^{\max }$ is thus defined more precisely as the smallest positive value of $B_{\mu}$ for which the vevs of the $A$ sector are zero. Fortunately, a sufficient condition for $B_{\mu}^{\max }$ to exist, which is that the vevs of the $A$ sector settle to 0 for large $B_{\mu}$, is easily satisfied and given by

$$
\frac{\alpha_{1}}{\lambda_{1}}+\frac{\alpha_{2}}{\lambda_{2}}+\frac{\alpha_{1} \alpha_{2}}{2 \lambda_{1} \lambda_{2}}>0
$$

When this relation is close to being satisfied but not quite, it is possible that the system falls in the scenario where the vevs in the $A$ sector are 0 for an interval but become positive again for large $B_{\mu}$.

This relation comes from looking at the limit of large $B_{\mu}$, where the $\mu_{i}^{2}$ 's can be ignored. In this case, setting $\alpha_{2}$ to 0 will result in the potential being minimized for both $\theta_{i}$ 's being $\pi / 4$. Increasing the magnitude of $\alpha_{2}$ while keeping the other parameters fixed will cause both angles to eventually start decreasing. There is always 
an extremum at $\theta_{1}=\theta_{2}=0$ and

$$
f_{1}=\frac{B_{\mu}^{1 / 2}}{\sqrt{2}\left(\lambda_{1}^{3} \lambda_{2}\right)^{1 / 8}}, \quad f_{2}=\frac{B_{\mu}^{1 / 2}}{\sqrt{2}\left(\lambda_{1} \lambda_{2}^{3}\right)^{1 / 8}}
$$

Minimizing around this point with respect to the angles leads to the set of equation

$$
\begin{aligned}
\left(\alpha_{1} f_{1}^{3}+B_{\mu} f_{2}\right) \theta_{1}-B_{\mu} f_{2} \theta_{2} & =0, \\
-B_{\mu} f_{1} \theta_{1}+\left(\alpha_{2} f_{2}^{3}+B_{\mu} f_{1}\right) \theta_{2} & =0 .
\end{aligned}
$$

The extremum at $\theta_{1}=\theta_{2}=0$ then becomes a minimum when

$$
\left(\alpha_{1} f_{1}^{3}+B_{\mu} f_{2}\right)\left(\alpha_{2} f_{2}^{3}+B_{\mu} f_{1}\right)-B_{\mu}^{2} f_{1} f_{2}>0
$$

Substituting the values of $f_{1}$ and $f_{2}$ of Eq. (4.24) leads to Eq. (4.23).

Alternate proof of Eq. (4.19). The expression for $B_{\mu}^{\max }$ can be obtained by a different procedure. Consider the requirement of Eq. (4.26) which remains true in general. Replacing the inequality by an equality and $B_{\mu}$ by $B_{\mu}^{\max }$ leads to an equation. Solving this equation for $B_{\mu}^{\max }$ in the limit of $f_{1}$ and $f_{2}$ constant gives the result of Eq. (4.19).

In principle, this technique can be used to obtain an exact value for $B_{\mu}^{\max }$. However, one must then take into account the dependence of $f_{1}$ and $f_{2}$ on $B_{\mu}$. This dependence can be obtained by minimizing the potential with respect to $f_{1}$ and $f_{2}$ in the small angles limit. These equations can be solved analytically for $f_{1}$ and $f_{2}$. However, they are coupled cubic equations and the solution is very cumbersome. Substituting these expressions in Eq. (4.26) and solving for $B_{\mu}^{\max }$ would then give an exact value for $B_{\mu}^{\max }$, but the complexity of $f_{1}$ and $f_{2}$ as a function of $B_{\mu}$ makes this calculation practically unfeasible. 
Nature of the lightest $C P$-even boson. When $B_{\mu}=0$ the pseudo-Goldstone boson from $H_{1}$ is an equal combination of the $A$ and $B$ sector, while the one from $H_{2}$ is purely in the $A$ sector. One would then expect that turning on $B_{\mu}$ would cause the resulting light pseudo-Goldstone boson to be more $A$-like than in the equivalent case for Twin Higgs. This turns out to be the case. To see this, we decompose the lightest pseudo-Goldstone as

$$
h=a h_{1 A}+b h_{2 A}+c h_{1 B}+d h_{2 B},
$$

where $h_{1 A}$ is defined via $H_{1 A}^{0}=v_{1 A}+\left(h_{1 A}+i A_{1 A}\right) / \sqrt{2}$ and identically for the other $h_{i}$ 's. The parameter $\Theta_{B} \equiv c^{2}+d^{2}$ represents a measure of how much the Higgs is $B$-like. A similar quantity can easily be defined for the Twin Higgs. The comparison for both models can be seen in Fig. 4.5. Note that the pseudo-Goldstone is most $A$ like for large mixing between $H_{1}$ and $H_{2}$. The price to pay for this is that constraints akin to those in the usual two Higgs doublet model become important. Fortunately, these constraints can easily be avoided, as the model naturally leads to a hierarchy between the vevs in the $A$ sector and fairly little mixing with mirror sector Higgses. Generally speaking, this means that our model will be better at avoiding constraints on Higgs couplings, though a full study of this is beyond the scope of this thesis.

Higgs particle spectrum. The particle spectrum in the $A$ sector is the usual two Higgs doublet model one. Generically speaking, creating a small vev hierarchy will push the masses of the heavier Higgses up for a fixed value of the lightest Higgs. The constraints from heavy scalar searches can therefore be easily avoided.

Mass ratios. A peculiar characteristic of the model is that generally $\left\langle H_{1 A}\right\rangle>$ $\left\langle H_{2 A}\right\rangle$ while $\left\langle H_{1 B}\right\rangle<\left\langle H_{2 B}\right\rangle$. In terms of standard two Higgs doublet model notation, this means that $\tan \beta_{A} \equiv\left\langle H_{1 A}\right\rangle /\left\langle H_{2 A}\right\rangle>1$ while $\tan \beta_{B} \equiv\left\langle H_{1 B}\right\rangle /\left\langle H_{2 B}\right\rangle<1$. This 


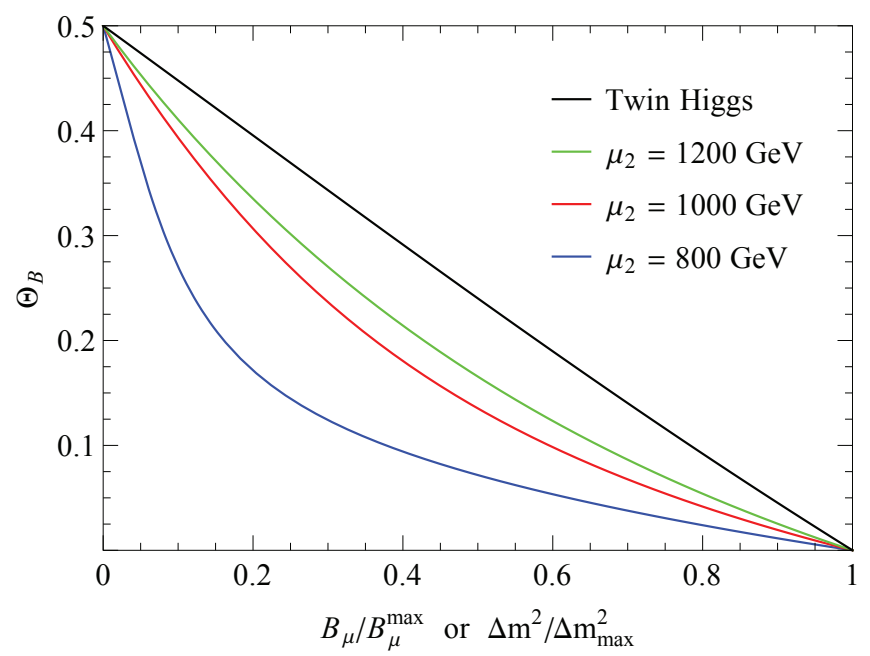

Figure 4.5: Example of $\Theta_{B}$ for the Twin Higgs and spontaneous $\mathbb{Z}_{2}$ breaking model with different values of $\mu_{2}$. The parameters for the Twin Higgs model are $\mu=750$ $\mathrm{GeV}, \alpha=-0.15$ and $\lambda=1$. The parameters for the spontaneous $\mathbb{Z}_{2}$ breaking model are $\mu_{1}=750 \mathrm{GeV}, \alpha_{1}=-0.15, \alpha_{2}=0.2$ and $\lambda_{1}=\lambda_{2}=1$.

property is specially interesting in a type II two Higgs doublet model, where $H_{1}$ couples to up-type quarks and $H_{2}$ to down-type quarks. In this case, the mirror down-type quarks will be heavier than their counterpart by $\left\langle H_{2 B}\right\rangle /\left\langle H_{2 A}\right\rangle$. Looking at Fig. 4.1, we see that it is the largest possible ratio of component vevs. As such, it is easy to make the mirror down-type quarks many times heavier than their counterpart. On the other hand, mirror up-type quarks are expected to be $\left\langle H_{1 B}\right\rangle /\left\langle H_{1 A}\right\rangle$ heavier, which is always a smaller ratio than for the down-type quarks. In addition, mirror massive gauge bosons are a factor of $\left(\left(\left\langle H_{1 B}\right\rangle^{2}+\left\langle H_{2 B}\right\rangle^{2}\right) /\left(\left\langle H_{1 A}\right\rangle^{2}+\left\langle H_{2 A}\right\rangle^{2}\right)\right)^{1 / 2}$ heavier than their counterpart. This ratio is always less than for down-type quarks, but larger than for up-type quarks. It is still easy to make mirror massive gauge bosons very heavy. In summary, a characteristic signature of our model with type II Yukawas is that the ratios of masses between a mirror particle and its counterpart is largest for down-type quarks and smallest for up-type quarks, while the ratio for massive gauge bosons is between these. 
Additional terms in the potential. The potential of Section (4.1.2) does not contain all possible $\mathbb{Z}_{2}$ preserving terms. It was verified that these extra terms do not affect the qualitative behavior of the system, as long as they are not much bigger than the terms already included. Even small explicit $\mathbb{Z}_{2}$ breaking terms do not affect the qualitative behavior of the system. Unless stated otherwise, such terms will be ignored from now on to avoid obscuring the analysis.

\subsubsection{Radiative corrections}

This section presents the one-loop leading radiative corrections to the parameters of the scalar potential for both the Twin Higgs and the spontaneous $\mathbb{Z}_{2}$ breaking Twin Higgs. Due to the similarities between these two models, the radiative corrections are nearly identical for the two. The main differences result from the Twin Higgs only having a single $S U(4)$ fundamental while the spontaneous $\mathbb{Z}_{2}$ breaking Twin Higgs contains two. These results are also similar to the radiative corrections given in Ref. [56], another Twin Higgs model with two $S U(4)$ fundamentals. The differences between their radiative corrections and the ones from this section follow from different forms of the quartic interactions.

To compute the radiative corrections, it is necessary to specify how the top couples to the different Higgses. In the Twin Higgs, this is encoded in the Lagrangian

$$
\mathcal{L}_{\text {top }}=-y_{t}\left(\bar{q}_{A} \tilde{H}_{A} t_{A}^{c}+\bar{q}_{B} \tilde{H}_{B} t_{B}^{c}\right)+\text { h.c. }
$$

where the $B$ sector quarks $q_{B}$ and $t_{B}^{c}$ do not carry Standard Model color and the tilde

notation stands for $\tilde{H}=i \sigma_{2} H^{*}$. The other Yukawa couplings can be safely ignored. 
The leading radiative corrections to the parameters of the Twin Higgs are then

$$
\begin{aligned}
\delta \mu^{2} & =\frac{1}{16 \pi^{2}}\left(6 y_{t}^{2}-\frac{9}{4} g^{2}-\frac{3}{4} g^{\prime 2}-10 \lambda-2 \alpha\right) \Lambda^{2}, \\
\delta \lambda & =\frac{1}{16 \pi^{2}}\left(6 y_{t}^{4}-\frac{9}{8} g^{4}-\frac{3}{4} g^{2} g^{\prime 2}-\frac{3}{8} g^{\prime 4}-32 \lambda^{2}-8 \lambda \alpha-2 \alpha^{2}\right) \ln \frac{\Lambda}{f}, \\
\delta \alpha & =\frac{1}{16 \pi^{2}}\left(-12 y_{t}^{4}+\frac{9}{4} g^{4}+\frac{3}{2} g^{2} g^{\prime 2}+\frac{3}{4} g^{\prime 4}-24 \lambda \alpha\right) \ln \frac{\Lambda}{f}, \\
\delta \Delta m^{2} & =\frac{1}{16 \pi^{2}}(-4 \lambda+4 \alpha) \Delta m^{2} \ln \frac{\Lambda}{f},
\end{aligned}
$$

where $y_{t}$ is the top Yukawa coupling, $g$ and $g^{\prime}$ are the SM gauge couplings and $\Lambda$ denotes the cutoff scale of the theory.

For the spontaneous $\mathbb{Z}_{2}$ breaking Twin Higgs, it is also necessary to specify how the top sector couples to the various Higgses. The top is chosen to couple to $H_{1}$ only and to follow the structure of Eq. (4.28). The radiative corrections also depend on how the down-type quarks and the charged lepton couple to the Higgses, but the size of their Yukawa couplings makes these contributions irrelevant.

Another difference between the spontaneous $\mathbb{Z}_{2}$ breaking Twin Higgs and the Twin Higgs is that, in the proposed model, radiative corrections also generate an additional operator of the form

$$
-\kappa\left(H_{1 A}^{\dagger} H_{1 A} H_{2 A}^{\dagger} H_{2 A}+H_{1 B}^{\dagger} H_{1 B} H_{2 B}^{\dagger} H_{2 B}\right)
$$

As mentioned above, the presence of a such a term does not modify qualitatively the behavior of the potential, as long as its coefficient is sufficiently small. It was verified that this is the case for the operator of Eq. (4.33) with a coefficient of the size of its radiative correction. Even a considerably larger coefficient does not affect the behavior much. Because of this, only its radiative correction is written and it is 
ignored afterward. The leading radiative corrections then take the form

$$
\begin{aligned}
\delta \mu_{1}^{2} & =\frac{1}{16 \pi^{2}}\left(6 y_{t}^{2}-\frac{9}{4} g^{2}-\frac{3}{4} g^{\prime 2}-10 \lambda_{1}-2 \alpha_{1}\right) \Lambda^{2}, \\
\delta \lambda_{1} & =\frac{1}{16 \pi^{2}}\left(6 y_{t}^{4}-\frac{9}{8} g^{4}-\frac{3}{4} g^{2} g^{\prime 2}-\frac{3}{8} g^{\prime 4}-32 \lambda_{1}^{2}-8 \lambda_{1} \alpha_{1}-2 \alpha_{1}^{2}\right) \ln \frac{\Lambda}{f_{1}}, \\
\delta \alpha_{1} & =\frac{1}{16 \pi^{2}}\left(-12 y_{t}^{4}+\frac{9}{4} g^{4}+\frac{3}{2} g^{2} g^{\prime 2}+\frac{3}{4} g^{\prime 4}-24 \lambda_{1} \alpha_{1}\right) \ln \frac{\Lambda}{f_{1}}, \\
\delta \mu_{2}^{2} & =\frac{1}{16 \pi^{2}}\left(-\frac{9}{4} g^{2}-\frac{3}{4} g^{\prime 2}-10 \lambda_{2}-2 \alpha_{2}\right) \Lambda^{2}, \\
\delta \lambda_{2} & =\frac{1}{16 \pi^{2}}\left(-\frac{9}{8} g^{4}-\frac{3}{4} g^{2} g^{\prime 2}-\frac{3}{8} g^{\prime 4}-32 \lambda_{2}^{2}-8 \lambda_{2} \alpha_{2}-2 \alpha_{2}^{2}\right) \ln \frac{\Lambda}{f_{2}}, \\
\delta \alpha_{2} & =\frac{1}{16 \pi^{2}}\left(\frac{9}{4} g^{4}+\frac{3}{2} g^{2} g^{\prime 2}+\frac{3}{4} g^{\prime 4}-24 \lambda_{2} \alpha_{2}\right) \ln \frac{\Lambda}{f_{2}}, \\
\delta B_{\mu} & =0, \\
\delta \kappa & =\frac{1}{16 \pi^{2}}\left(-\frac{9}{4} g^{4}-\frac{3}{2} g^{2} g^{\prime 2}-\frac{3}{4} g^{\prime 4}\right) \ln \frac{\Lambda}{f_{1}} .
\end{aligned}
$$

For all radiative corrections presented above, finite contributions were neglected.

\subsection{Numerical analysis of the fine-tuning}

In this section, the fine-tuning of the spontaneous $\mathbb{Z}_{2}$ breaking Twin Higgs is compared more precisely to that of the Twin Higgs. For both models, the fine-tuning comes from requesting a small $v / f$. In the case of the Twin Higgs, one has to tune the $\mathbb{Z}_{2}$ breaking sector against the $S U(4)$ breaking sector. The tuning is evaluated in a similar way to Ref. [61] by defining

$$
\Delta_{\mathrm{TH}}=\left|\frac{\partial \ln \left(v^{2} / f^{2}\right)}{\partial \ln \Delta m^{2}}\right| .
$$


The tuning is then $\Delta_{\mathrm{TH}}^{-1}$. There are however a number of constraints that need to be satisfied. The vev $v$ and the mass of the lightest Higgs must be adjusted to their correct values, which we take to be $174.10 \mathrm{GeV}$ [16] and $125.09 \mathrm{GeV}$ [62] respectively. In addition, $f / v$ must be large enough to avoid experimental constraints. Setting this ratio to a given value imposes an additional constraint. Alternatively, one can set the fine-tuning to a given number and be interested in $f / v$, which can be used to estimate the cutoff.

There are four parameters in the Twin Higgs potential: $\mu^{2}, \lambda, \alpha$ and $\Delta m^{2}$. Matching $v$ and the mass of the Higgs with their respective values sets two parameters. Fixing $f / v$ or the tuning determines another one. We are therefore left with a single free parameter. For convenience sake, that parameter is taken to be $\lambda$. Two benchmarks are given. First, setting $\lambda=1$ and $f / v=3$ leads to a tuning of $27.7 \%$. Second, setting $\lambda=1$ and requesting a tuning of $20 \%$ leads to a $f / v$ of 3.42 .

A similar measure of fine-tuning can be defined in the spontaneous $\mathbb{Z}_{2}$ breaking Twin Higgs, but a few differences need to be taken into account. First, $B_{\mu}$ plays a similar role to $\Delta m^{2}$. As explained in Section 4.1.2, one can obtain a very large ratio of vevs for a relatively small $B_{\mu} / B_{\mu}^{\max }$, given a very large mixing of $H_{1}$ and $H_{2}$. This however requires a fine-tuning of the parameters of the second Higgs $\left(\mu_{2}^{2}, \lambda_{2}\right.$ and $\left.\alpha_{2}\right)$ against those of the first. This tuning corresponds to $\Omega$ being close to 1 and needs to be taken into account. Second, there are simply more parameters in our case than in the original Twin Higgs. A measure that addresses all of these issues in a relatively fair manner is

$$
\Delta_{\text {Spontaneous }}=\operatorname{Max}\left\{\left|\frac{\partial \ln \left(v^{2} / f_{1}^{2}\right)}{\partial \ln B_{\mu}}\right|,\left|\frac{\partial \ln \left(v^{2} / f_{1}^{2}\right)}{\partial \ln \mu_{2}^{2}}\right|,\left|\frac{\partial \ln \left(v^{2} / f_{1}^{2}\right)}{\partial \ln \lambda_{2}}\right|,\left|\frac{\partial \ln \left(v^{2} / f_{1}^{2}\right)}{\partial \ln \alpha_{2}}\right|\right\} .
$$


The tuning is then $\Delta_{\text {Spontaneous. }}^{-1}{ }^{3}$ The number of parameters in the model is $7\left(\mu_{1}^{2}\right.$, $\mu_{2}^{2}, \lambda_{1}, \lambda_{2}, \alpha_{1}, \alpha_{2}$ and $\left.B_{\mu}\right)$. Three of them can be used to obtain the correct value of $v$ and the Higgs mass, as well as specifying $f_{1} / v$ or requesting a given tuning. A convenient choice is to use $\mu_{1}^{2}, \alpha_{1}$ and $B_{\mu}$ for this. The free parameters are then $\lambda_{1}$ and the parameters related to $\mathrm{H}_{2}$ only. For convenience, all of the following plots are shown for $\lambda_{1}=\lambda_{2}=1$. There are then two parameters left: $\mu_{2}^{2}$ and $\alpha_{2}$. As it makes the relation with the results of Section 4.1.2 clearer, all contour plots are presented in terms of $\mu_{2}^{2} / \mu_{1}^{2}$ and $-\alpha_{2} / \alpha_{1}$.

The left panel of Fig. 4.6 shows the tuning given a ratio $f_{1} / v$ of 3 . By inspecting Eq. (4.17), one sees that the contour lines correspond roughly to lines of constant $\Omega$. The tuning also approaches a constant as $\Omega$ goes to 0 . This corresponds to the behavior expected from the discussion of Section 4.1.2. The gray area corresponds to the region of parameter space where the constraints do not accept any solution. It originates from the impossibility of creating a large enough hierarchy of vevs for $\Omega$ very close to 1 . The model is least fine-tuned when $\Omega$ is large enough for feedback to play an important role, while at the same time far away enough from 1 not to be considered fine-tuned. The ratio of the tuning and the corresponding Twin Higgs benchmark of $27.7 \%$ is shown in the right panel of Fig. 4.6. There is an optimal improvement of $58.1 \%$ and an improvement of $29.2 \%$ in the limit of $\Omega$ going to 0 . Conversely, Fig. 4.7 shows $f_{1} / v$ for a fixed tuning of $20 \%$. The ratio of $f_{1} / v$ on the corresponding Twin Higgs benchmark of 3.42 can be seen in the right panel of Fig. 4.7. There is an optimal improvement of $22.5 \%$ and an improvement of $12.3 \%$ in the limit of $\Omega$ going to 0 .

\footnotetext{
${ }^{3}$ As in Ref. [61], variations with respect to $\mu_{1}^{2}, \alpha_{1}$ and $\lambda_{1}$ are not considered and they are taken to be fixed. Variations with respect to these parameters lead to slightly larger tuning, which is a consequence of $B_{\mu}^{\max }$ having cubic dependence on $f_{1}$. The chosen measure of tuning instead measures how close $B_{\mu}$ must be taken to $B_{\mu}^{\max }$ and how much the parameters of $H_{2}$ are adjusted with respect to those of $H_{1}$.
} 

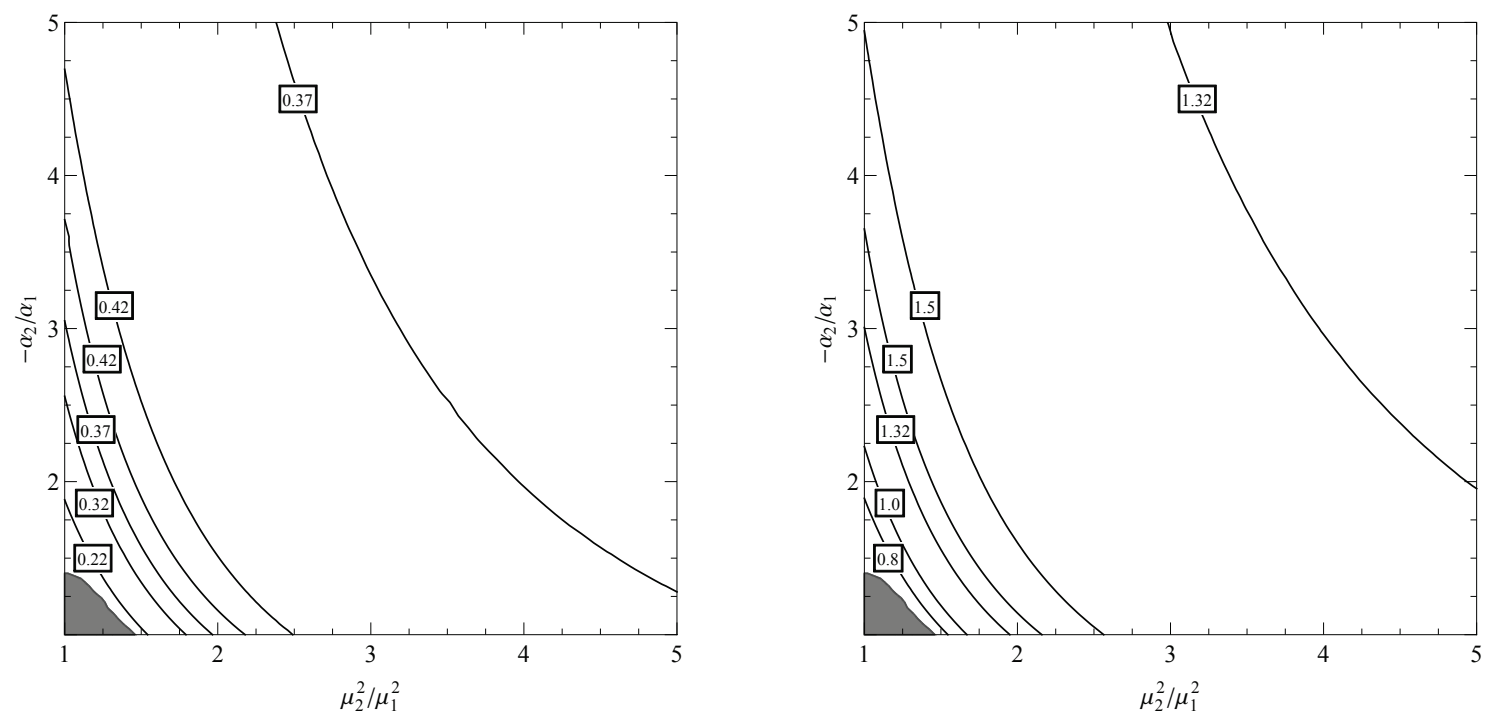

Figure 4.6: Tuning of the spontaneous $\mathbb{Z}_{2}$ breaking Twin Higgs for a fixed $f_{1} / v$ of 3. The left panel shows the tuning in percentage and the right one the ratio of the tuning to the Twin Higgs benchmark value of $27.7 \%$. The gray area corresponds to the region where the constraints do not accept any solution.
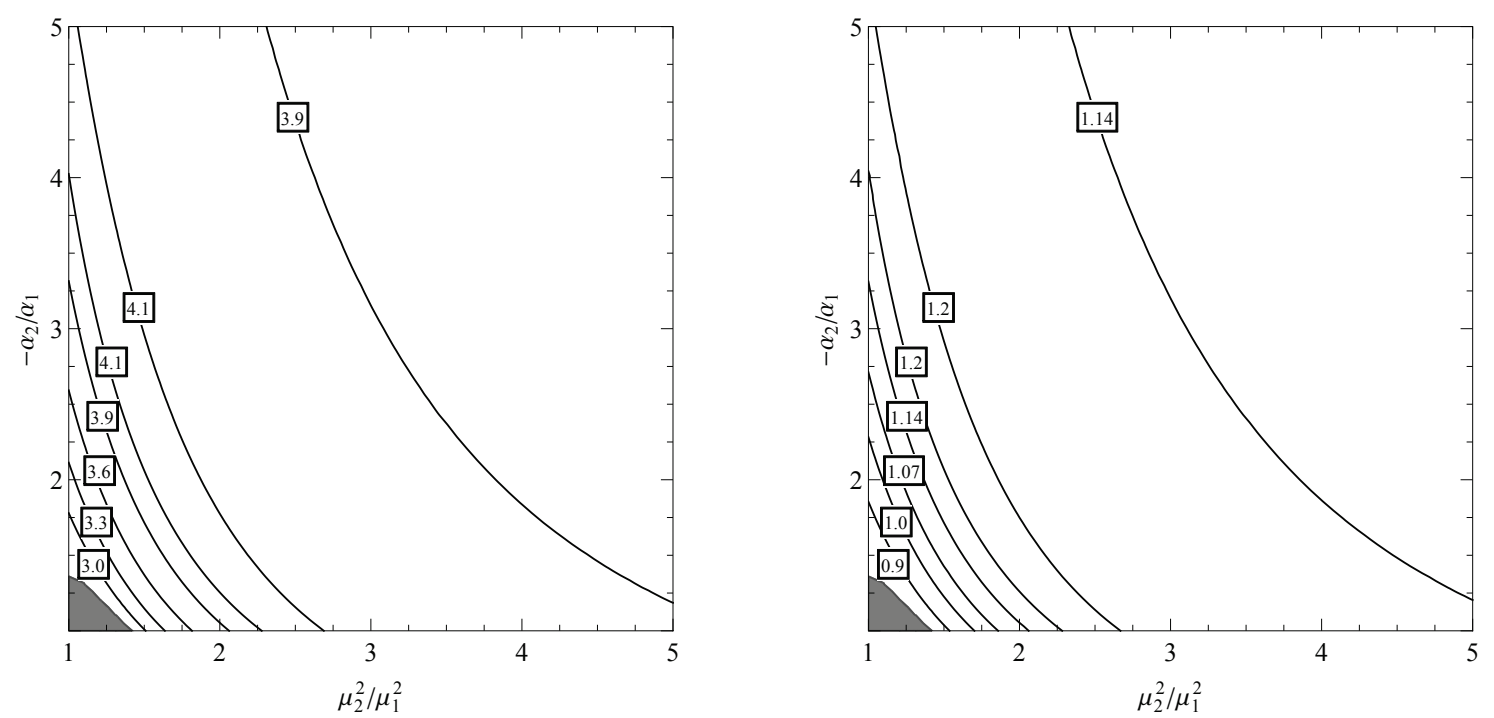

Figure 4.7: $f_{1} / v$ for the spontaneous $\mathbb{Z}_{2}$ breaking Twin Higgs for a fixed tuning of $20 \%$. The left panel shows $f_{1} / v$ and the right one the ratio of $f_{1} / v$ to the Twin Higgs benchmark value of 3.42. The gray area corresponds to the region where the constraints do not accept any solution. 
Also of interest is the scale at which new physics is expected to become relevant, i.e. the cutoff. New physics is expected where the radiative corrections to the different parameters become large compared to their actual values. The bare parameters must then be tuned against their radiative corrections. The relevant parameters in both Twin Higgs and our model are those that receive quadratic corrections, i.e. the different $\mu^{2}$ 's. An estimate of the cutoff for a fixed tuning can be obtained by taking the value of $\Lambda$ for which the ratio of one of the $\mu^{2}$ 's and its radiative correction drops below said tuning. These corrections are only expected to give the correct order of

magnitude and are roughly given by $\left|\delta \mu^{2}\right| \sim 10 \lambda^{2} \Lambda^{2} /\left(16 \pi^{2}\right)$. Using the relations of Section 4.1 , the results of $f / v$ can be used to estimate the cutoff. Requesting a tuning of $20 \%$ gives a cutoff of $7.5 \mathrm{TeV}$ for the Twin Higgs. In our model, the cutoff follows a similar pattern to Fig. 4.7 with an optimal value of $9.2 \mathrm{TeV}$ and a value of $8.4 \mathrm{TeV}$ in the limit of $\Omega$ going to 0 .

\subsection{Possible UV completion}

The next logical question concerns a possible UV completion. The obvious guess would be a supersymmetric version of the model. However, SUSY generally leads to a more complicated quartic structure than Eqs. (4.11) and (4.12). This prevents the model from being translated directly to SUSY. In addition, getting the correct signs of the $\alpha_{i}$ 's generally proves to be problematic. The combination of the D-terms and the largest loop corrections provides a negative contribution to the $\alpha_{i}$ of both the up and down Higgses [61]. The terms leading to spontaneous $\mathbb{Z}_{2}$ breaking must therefore originate from the superpotential. One possibility would be to introduce a 
superpotential term of the form

$$
\lambda H_{d A} U H_{d B}
$$

where $U$ is a fundamental of both $S U(2)_{A}$ and $S U(2)_{B}$ and has the appropriate weak hypercharges. Assuming a very large soft mass for $U$ and integrating it out would lead to a positive contribution to $\alpha_{d}$ and can lead to the correct $\mathbb{Z}_{2}$ breaking structure.

One other possibility would be to have both $H_{u}$ and $H_{d}$ preserve $\mathbb{Z}_{2}$, but include a NMSSM-like scalar sector that spontaneously breaks $\mathbb{Z}_{2}$. For example, consider the superpotential

$$
W=\lambda^{\prime} S^{\prime}\left(S_{A}^{2}+S_{B}^{2}\right)+\lambda^{\prime \prime} S^{\prime \prime} S_{A} S_{B}
$$

and assume that both $S^{\prime}$ and $S^{\prime \prime}$ have large soft masses and that $S=\left(S_{A}, S_{B}\right)$ has a negative soft mass squared. The first term preserves a global $O(2)$ symmetry that the second term breaks. Both terms preserve the $\mathbb{Z}_{2}$ symmetry. However, this symmetry is broken spontaneously. If $S_{A}$ couples to the $A$-type Higgses and $S_{B}$ to the $B$-type Higgses, the symmetry breaking is transmitted to the Higgs sector as well. Of course, the viability of these models would require studies of their own. 


\section{Chapter 5}

\section{Constraining Mini-Split}

\section{Supersymmetry}

As explained in Chapter 3, Supersymmetry presents many interesting features. The most prominent ones are that it solves the hierarchy problem, can provide a viable dark matter candidate and leads to gauge coupling unification. As such, many dedicated searches have been performed in the hope of discovering superpartners. These searches have been unsuccessful so far, resulting in stringent constraints on several superpartners. These constraints are particularly strong for scalar partners and make the stabilization of the weak scale more challenging. One is then forced to reconsider whether nature might be fine-tuned to a certain degree. This has led to the emergence of Split-SUSY. In this setup, the fermion superpartners are around or close to the electroweak scale, while the scalar superpartners are multiple orders of magnitude heavier. The resulting mass spectrum can still provide a dark matter candidate and preserve gauge coupling unification, while avoiding the most stringent experimental constraints. The mass of the Higgs is however fine-tuned and must be explained by other means, such as an environmental selection principle. 
However, it was shown in Ref. [63] that pushing the scalar masses above $10^{5}$ $\mathrm{TeV}$ can make Split-SUSY incapable of accommodating the now known mass of the Higgs boson $[7,8]$. Split-SUSY must then be promoted to Mini-Split SUSY, where the gap between the masses of fermion and boson superpartners is only a few orders of magnitude.

One of the main phenomenological characteristics of Mini-Split models is the presence of a small energy gap between the gauginos and scalars. The conventional mass spectra associated to well known mediation mechanism like anomaly $[31,32]$ and gauge [25-30] mediation are modified, as the heavy superpartners deflect the gaugino masses from their standard renormalization group (RG) expressions when they are integrated out. The resulting spectra are referred to as deflected anomaly mediation $[64,65]$ and deflected gauge mediation.

The purpose of this chapter is to constrain the parameter space of Mini-Split models with deflected anomaly mediation and gauge mediation using LHC data and to predict future prospects for the $14 \mathrm{TeV}$ run of the LHC (LHC 14) and a future $100 \mathrm{TeV}$ collider. Current constraints are extracted from ATLAS [66-70] and CMS [3,71,72] SUSY searches (mainly gluino pair production), the mass of the Higgs boson $[7,8]$ and the absence of a color breaking vacuum. The same techniques in conjunction with background estimates can then be applied to obtain prospects for LHC 14 and a $100 \mathrm{TeV}$ collider. The focus in this chapter is on the case of a Higssino mass $\mu$ around the scalar scale. The Higgsinos are then the main origin of deflection and the light neutralinos/charginos are almost pure gauginos. Since the masses of the third generation squarks are expected to run down with decreasing energy, we make the simplifying assumption of a lighter third generation.

This chapter is organized as follows. The necessary theoretical elements are 
presented first. This includes an explanation of how Mini-Split theories can arise in both anomaly and gauge mediation, as well as pole mass expressions and branching fractions. The procedure necessary to calculate the Higgs mass is also presented. The methodology used in obtaining both current limits and future prospects is then explained. This includes the LHC searches used to determine current limits. We then present current LHC constraints and prospects at LHC 14 and a future $100 \mathrm{TeV}$ collider. Finally, we make a few comments concerning dark matter and disappearing tracks.

\section{$5.1 \quad$ Theory}

\subsubsection{Mini-Split models}

In this section, we review how Mini-Split spectra can be realized in both anomaly and gauge mediation (see for example Ref. [65]). Quite generally, sfermion masses can be generated via terms of the form

$$
\int d^{4} \theta \frac{X^{\dagger} X}{M_{*}^{2}} Q^{\dagger} Q
$$

where $M_{*}$ is the mediation scale, $X=\frac{1}{2} \theta^{2} F_{X}$ is a SUSY breaking spurion, and $Q$ is a chiral superfield (see Section 3.5). This term is always allowed by symmetries, irrespective of the R-charge of $X$ or its gauge quantum numbers. On the other hand gaugino masses are generated via terms of the form

$$
\int d^{2} \theta \frac{X}{4 M_{*}} \mathcal{W}_{i}^{\alpha} \mathcal{W}_{i \alpha}
$$


where $\mathcal{W}_{i \alpha}(i=1,2,3)$ are the gauge field strength superfields. Contrary to the sfermion masses of Eq. (5.1), here $X$ is required to be a singlet under all gauge and global charges in order for this term to be allowed. It is therefore easier to forbid and it is assumed absent in the models considered in this chapter. There is however an unavoidable contribution to gaugino masses coming from anomaly mediation (see Section 3.5.3),

$$
M_{i}=\frac{\beta_{i}}{g_{i}} m_{3 / 2}
$$

The A-terms are also generated by anomaly mediation and are given by

$$
A_{y}=-\beta_{y} m_{3 / 2}
$$

where $\beta_{y}$ is the beta function of the corresponding Yukawa coupling. A $B_{\mu}$ term can be generated by a term of the form

$$
\int d^{4} \theta \frac{X^{\dagger} X}{M_{*}^{2}} H_{u} H_{d}
$$

In Mini-Split scenarios, the $\mu$ term can either be large (at the scale of the scalars) or small (at the scale of the gauginos) depending on how it is generated. This chapter focuses on the case in which it is large. The $\mu$ term can be generated through the Giudice-Masiero mechanism [73] where a term of the following form is introduced,

$$
\int d^{4} \theta \Phi^{\dagger} \Phi\left[\hat{H}_{u, d}^{\dagger} \hat{H}_{u, d}+\left(c \hat{H}_{u} \hat{H}_{d}+\text { h.c }\right)\right]
$$

Here $c$ is an arbitrary dimensionless constant and $\Phi$ is the conformal compensator (see Section 3.5.3) which gets a non-zero $F$-term once SUSY is broken: $\Phi=1-m_{3 / 2} \theta^{2} / 2$. 
Upon rescaling of the fields, this becomes

$$
\int d^{4} \theta\left[H_{u, d}^{\dagger} H_{u, d}+\left(c \frac{\Phi^{\dagger}}{\Phi} H_{u} H_{d}+\text { h.c }\right)\right]
$$

and leads to a $\mu$ term, in addition to an additional contribution to $B_{\mu}$. These terms are of order $m_{3 / 2}$ and $m_{3 / 2}^{2}$ respectively. If gravity is the sole mediator of supersymmetry breaking, then $M_{*}$ is the Planck mass. This leads to the scalars and Higgsinos all having masses of roughly $m_{3 / 2}$ while the masses of the gauginos are a loop factor smaller. The resulting mass spectrum falls in the Mini-Split category.

The fact that the $\mu$ term is taken to be large will change the running of the gauge coupling constants compared to the more conventional split-spectrum with light Higgsinos and might affect unification. There exists a simple procedure to test whether gauge coupling unification can be maintained. Starting from the electroweak scale, the gauge coupling constants of $S U(2)_{L}$ and $U(1)_{Y}$ are evolved until they unify. The strong coupling constant at that scale is taken to be equal to the other ones. The strong coupling constant is then evolved down to the electroweak scale and compared with its experimental value. The prediction for $\alpha_{s}\left(M_{Z}\right)$ was found in Ref. [74] to be smaller than with light Higgsinos, but still consistent with the measured value.

Gauge mediation can also lead to Mini-Split spectra. This can be done in a multitude of ways. An example taken from Ref. [63] is presented here. Assume a superpotential of the form

$$
W=M_{R}\left(\Phi_{1} \bar{\Phi}_{1}+\Phi_{2} \bar{\Phi}_{2}\right)+X \Phi_{1} \bar{\Phi}_{2}
$$

where the $\Phi_{i}$ and the $\bar{\Phi}_{i}$ are messengers and $X=M+\frac{1}{2} F \theta^{2}$ is a spurion that breaks 
SUSY and R-symmetry. This leads to gaugino masses of

$$
M_{i}=\frac{\alpha_{i}}{6 \pi} \frac{M}{M_{R}} \frac{F^{3}}{M_{R}^{5}}+\mathcal{O}\left(\frac{M^{3}}{M_{R}^{3}} \frac{F^{3}}{M_{R}^{5}}, \frac{F^{5}}{M_{R}^{9}}\right) .
$$

On the other hand, the scalar masses are $\mathcal{O}\left(\alpha F / M_{R}\right)$. If R-symmetry is weakly broken $\left(M<M_{R}\right)$, a Mini-Split spectrum is again generated.

\subsubsection{Gaugino mass spectrum}

The main effect of the small mass hierarchy between the gauginos and scalars/Higgsinos is that radiative corrections to the pole masses of gauginos coming from integrating out the scalars and Higgsinos can be comparable to, if not larger than, the contributions coming from anomaly mediation or gauge mediation directly. In the case of anomaly mediation, the expressions are well known and can be read from different sources $[65,75]$. In the limit of degenerate sfermion masses, the pole masses of the gauginos are

$$
\begin{aligned}
M_{\tilde{B}}=M_{1}(Q) & {\left[1+\frac{C_{\mu}}{11}+\frac{8 g_{1}^{2}}{80 \pi^{2}}\left(-\frac{41}{2} \ln \frac{Q^{2}}{M_{1}^{2}}-\frac{1}{2} \ln \frac{\mu^{2}}{M_{1}^{2}}+\ln \frac{m_{A}^{2}}{M_{1}^{2}}+11 \ln \frac{m_{\tilde{q}}^{2}}{M_{1}^{2}}\right.\right.} \\
& \left.\left.+9 \ln \frac{m_{\tilde{l}}^{2}}{M_{1}^{2}}\right)+\frac{g_{3}^{2}}{6 \pi^{2}}-\frac{13 g_{t}^{2}}{264 \pi^{2} \sin ^{2} \beta}\right], \\
M_{\tilde{W}}=M_{2}(Q) & {\left[1+C_{\mu}+\frac{g_{2}^{2}}{16 \pi^{2}}\left(\frac{19}{6} \ln \frac{Q^{2}}{M_{2}^{2}}-\frac{1}{6} \ln \frac{\mu^{2}}{M_{2}^{2}}+\frac{1}{3} \ln \frac{m_{A}^{2}}{M_{2}^{2}}+3 \ln \frac{m_{\tilde{q}}^{2}}{M_{2}^{2}}\right.\right.} \\
& \left.\left.+\ln \frac{m_{\tilde{l}}^{2}}{M_{2}^{2}}\right)+\frac{3 g_{3}^{2}}{2 \pi^{2}}-\frac{3 g_{t}^{2}}{8 \pi^{2} \sin ^{2} \beta}\right], \\
M_{\tilde{G}}=M_{3}(Q) & {\left[1+\frac{g_{3}^{2}}{16 \pi^{2}}\left(7 \ln \frac{Q^{2}}{M_{3}^{2}}+4 \ln \frac{m_{\tilde{q}}^{2}}{M_{3}^{2}}+13-2 F\left(\frac{M_{3}^{2}}{m_{\tilde{q}}^{2}}\right)\right)-\frac{7 g_{3}^{2}}{24 \pi^{2}}\right.} \\
& \left.+\frac{g_{t}^{2}}{12 \pi^{2} \sin ^{2} \beta}\right],
\end{aligned}
$$


where

$$
M_{1}(Q)=\frac{33 g_{1}^{2}(Q)}{80 \pi^{2}} m_{3 / 2}, \quad M_{2}(Q)=\frac{g_{2}^{2}(Q)}{16 \pi^{2}} m_{3 / 2}, \quad M_{3}(Q)=-\frac{3 g_{3}^{2}(Q)}{16 \pi^{2}} m_{3 / 2},
$$

$g_{i}(Q)$ are the gauge couplings of the $\mathrm{SM}$ in $\overline{M S}$ and $\mathrm{SU}(5)$ convention at scale $Q, g_{t}$ is the top Yukawa coupling in the SM, and

$$
\begin{aligned}
C_{\mu} & =\frac{\mu}{m_{3 / 2}} \frac{m_{A}^{2} \sin ^{2} \beta}{m_{A}^{2}-\mu^{2}} \ln \frac{m_{A}^{2}}{\mu^{2}}, \\
F(x) & =3\left[\frac{3}{2}-\frac{1}{x}-\left(\frac{1}{x}-1\right)^{2} \ln |1-x|\right] .
\end{aligned}
$$

The generalization to the case of non-degenerate sfermion masses is straight-forward.

The proof of these expressions is tedious but not overly complicated. It is therefore only summarized. We start by relating the pole masses to the $\overline{D R}$ masses using results from Ref. [76]. Corrections from two loop beta functions must be taken into account and can be read from Ref. [77]. Finally, the gauge coupling constants must be converted from $\overline{D R}$ to $\overline{M S}$ using the results of Ref. [65].

The main point of interest is that the corrections due to $C_{\mu}$ can be comparable to, if not bigger than, the usual expressions. A typical mass spectrum is shown in the left panel of Fig. 5.1. Similar expressions hold for gauge mediation:

$$
\begin{aligned}
M_{\tilde{B}}=M_{1}^{\prime}(Q) & {\left[1+\frac{3 C_{\mu}^{\prime}}{5}+\frac{g_{1}^{2}}{80 \pi^{2}}\left(-\frac{41}{2} \ln \frac{Q^{2}}{M_{1}^{2}}-\frac{1}{2} \ln \frac{\mu^{2}}{M_{1}^{2}}+\ln \frac{m_{A}^{2}}{M_{1}^{2}}+11 \ln \frac{m_{\tilde{q}}^{2}}{M_{1}^{2}}\right.\right.} \\
& \left.\left.+9 \ln \frac{m_{\tilde{l}}^{2}}{M_{1}^{2}}\right)\right], \\
M_{\tilde{W}}=M_{2}^{\prime}(Q) & {\left[1+C_{\mu}^{\prime}+\frac{g_{2}^{2}}{16 \pi^{2}}\left(\frac{19}{6} \ln \frac{Q^{2}}{M_{2}^{2}}-\frac{1}{6} \ln \frac{\mu^{2}}{M_{2}^{2}}+\frac{1}{3} \ln \frac{m_{A}^{2}}{M_{2}^{2}}+3 \ln \frac{m_{\tilde{q}}^{2}}{M_{2}^{2}}\right.\right.} \\
& \left.\left.+\ln \frac{m_{\tilde{l}}^{2}}{M_{2}^{2}}\right)\right],
\end{aligned}
$$




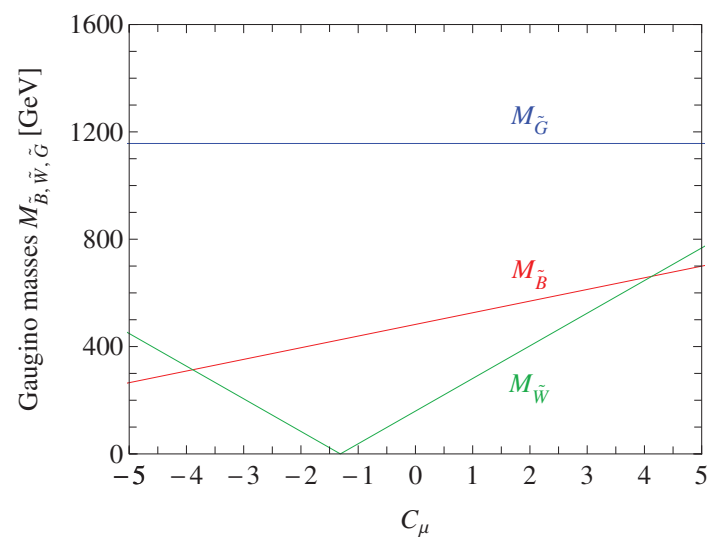

(a)

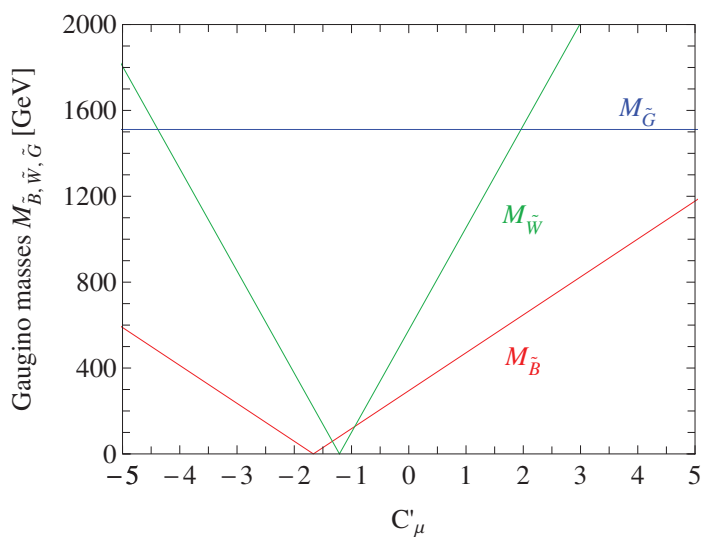

(b)

Figure 5.1: Typical mass spectrum for (a) anomaly mediation and (b) gauge mediation. In (a), the masses appearing on the right side of Eq. (5.10) are taken to be $m_{\text {scalars }}=\mu=m_{3 / 2}=50 \mathrm{TeV}$ with $\tan \beta=2$. In (b), the masses appearing on the right side of Eq. (5.13) are taken to be $m_{\text {scalars }}=\mu=\Lambda=200 \mathrm{TeV}$ with $\tan \beta=2$.

$$
M_{\tilde{G}}=M_{3}^{\prime}(Q)\left[1+\frac{g_{3}^{2}}{16 \pi^{2}}\left(7 \ln \frac{Q^{2}}{M_{3}^{2}}+4 \ln \frac{m_{\tilde{q}}^{2}}{M_{3}^{2}}+13-2 F\left(\frac{M_{3}^{2}}{m_{\tilde{q}}^{2}}\right)\right)+\frac{6 g_{3}^{2}}{16 \pi^{2}}\right] \text {, }
$$

where only the terms proportional to $g_{t}, g_{3}$, or log-enhanced [78] are kept,

$$
\begin{aligned}
& M_{i}^{\prime}(Q)=\frac{g_{i}^{2}}{16 \pi^{2}} \Lambda, \\
& C_{\mu}^{\prime}=\frac{\mu}{\Lambda} \frac{m_{A}^{2} \sin ^{2} \beta}{m_{A}^{2}-\mu^{2}} \ln \frac{m_{A}^{2}}{\mu^{2}},
\end{aligned}
$$

where $\Lambda$, in a given gauge mediation model, can be expressed in term of the SUSY breaking scale and the messenger scales (see for example Eq. (5.9)). The last term of $M_{\tilde{G}}$ in Eq. (5.13) is the two loop correction to the $\overline{D R}$ mass and can be extracted from Ref. [79]. A typical mass spectrum is shown in the right panel of Fig. 5.1.

The parameters $C_{\mu}$ and $C_{\mu}^{\prime}$ can be rewritten by requiring the fine-tuning condition, which needs to be imposed to have the weak scale parametrically smaller than 
the scalar mass scale [65]

$$
\tan ^{2} \beta=\frac{m_{H_{d}}^{2}+\mu^{2}}{m_{H_{u}}^{2}+\mu^{2}}
$$

and the usual relation $m_{A}^{2}=m_{H_{u}}^{2}+m_{H_{d}}^{2}+2 \mu^{2}$. $C_{\mu}$ can then be expressed as [65]

$$
C_{\mu}=\frac{2 \mu \tan \beta}{m_{3 / 2}} \frac{m_{H_{d}}^{2}+\mu^{2}}{\left(\tan ^{2} \beta+1\right) m_{H_{d}}^{2}+\mu^{2}} \ln \left[\left(1+\cot ^{2} \beta\right)\left(1+\frac{m_{H_{d}}^{2}}{\mu^{2}}\right)\right] .
$$

The same applies to $C_{\mu}^{\prime}$ with $m_{3 / 2} \rightarrow \Lambda$.

In these models the gauginos are the lightest sparticles and, because $\mu$ is large, neutralino mixing and chargino mixing are suppressed. The light neutralinos and

charginos are then almost pure binos and winos. As such, there is a neutralino of mass very close to $M_{\tilde{B}}$ and a pair of nearly degenerate neutralino and chargino of mass $M_{\tilde{W}}$. There is a small mass difference between the neutral and charged wino dominated by a loop effect [80],

$$
\Delta M \equiv m_{\chi_{\tilde{W}}^{+}}-m_{\chi_{\tilde{W}}^{0}}=\frac{\alpha_{2} M_{2}}{4 \pi}\left[f\left(r_{W}\right)-c_{W}^{2} f\left(r_{Z}\right)-s_{W}^{2} f\left(r_{\gamma}\right)\right],
$$

where $f(y)=\int_{0}^{1}(2+2 x) \log \left(x^{2}+(1-x) y^{2}\right) d x$ and $r_{i}=m_{i} / M_{2}$. The mass splitting is typically of the order of $150 \mathrm{MeV}$.

\subsubsection{Gaugino decays}

In this chapter, the gluino is assumed to decay via third generation squarks. These decay modes dominate if the third generation squarks are lighter than the others, which is expected from renormalization group $(\mathrm{RG})$ effects or could be imposed for 
other model building reasons. ${ }^{1}$ The relevant decays are then

$$
\begin{aligned}
& \tilde{g} \rightarrow t \bar{t} \chi_{1}^{0}, \quad \tilde{g} \rightarrow b \bar{b} \chi_{1}^{0}, \quad \tilde{g} \rightarrow b \bar{t} \chi_{1}^{+}, \\
& \tilde{g} \rightarrow t \bar{t} \chi_{2}^{0}, \quad \tilde{g} \rightarrow b \bar{b} \chi_{2}^{0}, \quad \tilde{g} \rightarrow \bar{b} t \chi_{1}^{-} .
\end{aligned}
$$

The gluino can also decay to a gluon and a neutralino; however, it is negligible for heavy enough Higgsinos [81] and it is ignored. The branching ratios are calculated using analytical results found in Ref. [82]. An example of gluino branching fractions is shown in Fig. 5.2. The computed branching ratios assume equal masses for the stops and the sbottoms. If the stops were lighter, the decays to two $b$ quarks, which can only proceed via off-shell sbottoms, would be relatively suppressed. As can be seen in Fig. 5.2, these decays are already suppressed. The only thing that would change is the branching fraction of $\tilde{g} \rightarrow t \bar{t} \chi_{1}^{0}, \tilde{g} \rightarrow t \bar{t} \chi_{2}^{0}$ and $\tilde{g} \rightarrow b \bar{t} \chi_{1}^{+}$, which all have similar efficiencies for the searches considered. It is therefore not expected that this assumption will affect the results greatly.

In practice, $\chi_{2}^{0}$ always decays to $\chi_{1}^{0}$ and a Higgs boson [74], irrespective of whether $M_{\tilde{B}}$ is larger than $M_{\tilde{W}}$ or the opposite. In this scenario, the decay of $\chi_{2}^{0}$ to $\chi_{1}^{0}$ and a $\mathrm{Z}$ boson is extremely suppressed due to the neutralinos being almost pure gauginos. When $M_{\tilde{W}}<M_{\tilde{B}}, \chi_{1}^{+}$can only decay to $\chi_{1}^{0}$ and either light leptons or a pion which can cause this chargino to be metastable because of lack of phase-space [80]. As the decay is always very soft, the decay products are generally unaccounted for in the detector and the chargino is practically indistinguishable from the stable neutralino. When $M_{\tilde{W}}>M_{\tilde{B}}, \chi_{1}^{+}$decays to $\chi_{1}^{0}$ and a $W$ boson (it was verified that the decay to $\chi_{2}^{0}$ only becomes relevant for $\mu$ at a scale considerably higher than anything relevant

\footnotetext{
${ }^{1}$ For example, flavour physics might require the first and second generations of squarks to be in the $1000 \mathrm{TeV}$ range, while the third generation could be kept somewhat lighter to obtain the appropriate Higgs mass [74].
} 


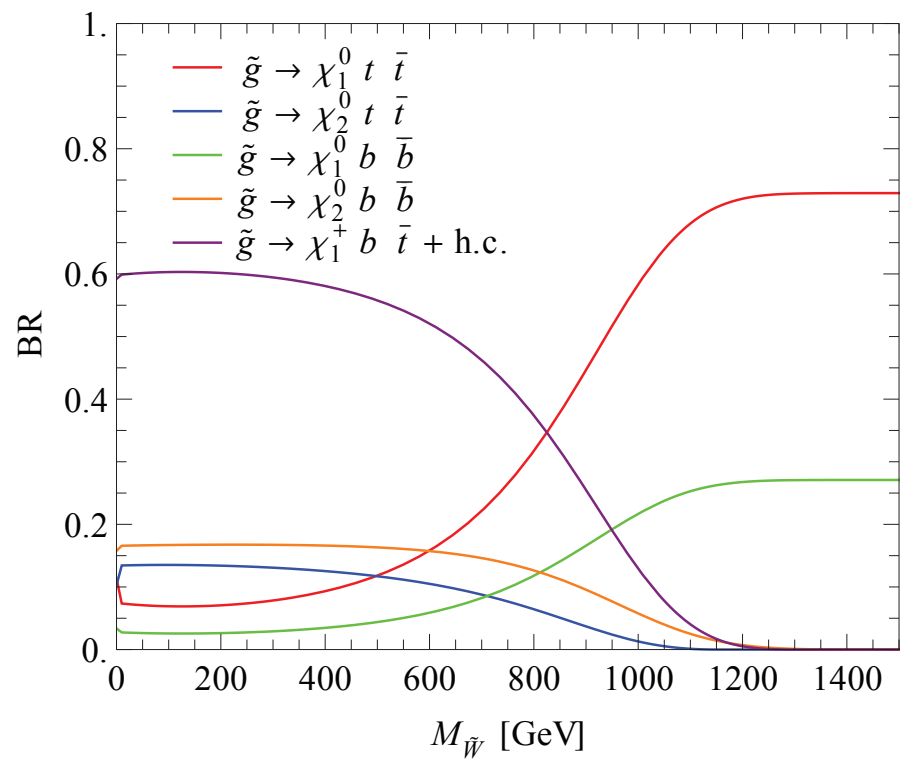

Figure 5.2: Branching ratios of the gluino for $M_{\tilde{G}}=1500 \mathrm{GeV}$ and $M_{\tilde{B}}=0 \mathrm{GeV}$. The third generation scalar masses are assumed to be degenerate and much heavier than the gauginos.

to this chapter).

\subsubsection{Higgs mass}

To set the mass of the Higgs to its experimentally measured value, the procedure outlined in Ref. [65] is used and summarized here. First, $\overline{M S}$ parameters are taken from Ref. [83] for the top Yukawa and the gauge coupling constants and from Ref. [84] for the bottom and tau Yukawas. The quartic coupling of the Higgs boson is extracted from its pole mass [85-88] using a value of $125.15 \mathrm{GeV}$, which is the naive average of the ATLAS [8] and CMS [7] values. These parameters are then evolved up to the scalars scale using three-loops beta functions [89-91]. Threshold corrections are taken from Ref. [65]. These include one-loop corrections and two-loop QCD corrections. The Higgs quartic is then matched with its SUSY expression and the threshold corrections. This determines one of the parameters, therefore reducing the 
dimension of the parameter space by one. As explained in the next section, $\tan \beta$ is varied to obtain the correct value of the Higgs mass.

In some regions of the parameter space, it is not possible to obtain the correct Higgs mass because the required parameters lead to a color breaking minimum that is deeper than the electroweak minimum. The necessary condition to avoid this is [65]

$$
\frac{\left(A_{t}-\mu y_{t} \cot \beta\right)^{2}}{y_{t}^{2} m_{Q_{3}} m_{U_{3}}}<\left(4-\frac{1}{\sin ^{2} \beta}\right)\left(\frac{m_{Q_{3}}^{2}}{m_{U_{3}}^{2}}+\frac{m_{U_{3}}^{2}}{m_{Q_{3}}^{2}}\right),
$$

where $m_{Q_{3}}$ is the third generation soft mass for the $\mathrm{SU}(2)$ quark doublet and $m_{U_{3}}$ the right-handed stop soft mass.

\subsection{Methodology and results}

\subsubsection{Parameter space}

We begin by discussing the parameter space we use to study the models of interest. It is very similar for both anomaly and gauge mediation. There are essentially four parameters that control the phenomenology of anomaly mediation [65]. They are $m_{3 / 2}, \tan \beta, m_{\text {scalars }}$, and $\mu$. As explained in Section 5.1.1, $m_{3 / 2}$ and $m_{\text {scalars }}$ are expected to be of the same order of magnitude so they are set equal to each other. An additional parameter can be fixed by requiring the theory to predict the correct mass of the Higgs boson with the help of the results of Section 5.1.4. Generally speaking, $\tan \beta$ is the best parameter to do so as varying it even slightly can have a substantial effect on the Higgs mass. The parameter space is then reduced to $\mu$ and $m_{3 / 2}$. However, we trade $\mu$ for $C_{\mu}$. The main advantage of this parametrization is that the ratio of gaugino masses depends mostly on $C_{\mu}$. The exact details of the scalar sector are relegated to two-loops corrections in Eq. (5.10) and the results can 
therefore be applied to models where the scalar sector does not differ too significantly. To translate this to something more familiar, each parameter space plot is provided with contours of constant $M_{\tilde{B}}, M_{\tilde{W}}, \mu$, and $\tan \beta$.

The relationship between $\mu$ and $C_{\mu}$ depends on $m_{H_{d}}$ which is taken to be at $m_{\text {scalars }}$. A different choice would lead, for the same $C_{\mu}$, to a different value of $\mu$ which in turn would affect mostly the color breaking bounds (see Eq. (5.19)). Taking $m_{H_{d}}$ much bigger than $m_{\text {scalars }}$ would limit $C_{\mu}$ to a narrow band around 0 and taking $m_{H_{d}}$ much smaller would push the bounds to large values of $C_{\mu}$ such that the gluino would be the LSP for most of the parameter space. With $m_{H_{d}}$ being set to $m_{\text {scalars }}$, we have a benchmark that does not suffer from any of these drawbacks. The third generation is assumed to be lighter than the others. As a benchmark, the first and second generation squark masses are set to $4 m_{\text {scalars }}$ and all third generation masses to $m_{\text {scalars }}$. This is small enough to prevent problems with large logs, while keeping the branching fraction to the first two generations below the percent level which is well below some of the uncertainties (e.g. gluino pair production cross section). Slepton masses are also set to $4 m_{\text {scalars }}$. Lowering the masses of the first two generations of squarks would increase the branching ratio of the gluino to light jets, possibly affecting the reach of our searches (however, the high jet-multiplicity would still provide strong bounds). It would have only a slight effect on the gaugino spectrum and on the Higgs mass. Finally, the third generation A-term $A_{t}$ is set by Eq. (5.4). Overall, changing the choice of benchmark parameters (mainly the choice of setting $m_{H_{d}}$ to $m_{\text {scalars }}$ and of taking $m_{Q_{3}}=m_{U_{3}}=m_{\text {scalars }}$ ) will mostly affect the $\mu$ and $\tan \beta$ contours in our results. Also, as a result of a modified relationship between $C_{\mu}, \mu$, and $\tan \beta$, the region of parameter space where there is a color breaking vacuum would be modified. In almost all of the parameter space the Higgsinos are heavy, except for a region 
near $C_{\mu}=0$ where a Higgsino can be the LSP. More precisely, outside of $\left|C_{\mu}\right|<0.3$, the Higgsinos are always an order of magnitude heavier than the gluino while only inside $\left|C_{\mu}\right|<0.1$ are the Higgsinos comparable in mass to the bino and winos. This represents only a very narrow band in the parameter space and the efficiencies of the signal regions are not expected to change much in it. In addition, this case has already been studied in Refs. [81,92]. As such, this effect is neglected. When $M_{\tilde{W}}<M_{\tilde{B}}$, the mass difference between $\chi_{1}^{+}$and $\chi_{1}^{0}$ is calculated using Eq. (5.17).

The previous discussion applies almost directly to gauge mediation by trading $m_{3 / 2}$ for $\Lambda$. In this case, $m_{\text {scalars }}$ is fixed to $\Lambda$ while $\tan \beta$ is again set by requesting the correct mass of the Higgs boson. ${ }^{2}$ The masses of the sleptons and the first two generations squarks are still set to $4 m_{\text {scalars }}$. $A_{t}$ is set to zero, as one would expect it to be small [65] and then completely overshadowed by $\mu$. The mass $m_{H_{d}}$ is once more set to $m_{\text {scalars }}$.

Two other constraints are of importance for the parameter space. First of all, for a given value of $m_{3 / 2}(\Lambda)$, a small value of $A_{t}$ will lead to an upper bound on $C_{\mu}\left(C_{\mu}^{\prime}\right)$ beyond which it is impossible to obtain the correct Higgs mass. Indeed if $C_{\mu}\left(C_{\mu}^{\prime}\right)$ becomes large, the threshold corrections also become large and the quartic matching condition does not accept any solutions for real $\tan \beta$. In fact, requiring $C_{\mu}\left(C_{\mu}^{\prime}\right)$ close to its upper bound can make the Higgsinos heavy enough that large logs could become a problem and perturbation expansions could fail. Fixing the stop mixing parameter $A_{t}-\mu y_{t} \cot \beta$ to a small value would solve this problem, but this would imply $A_{t}$ reaching values that are too high to be readily explained in our framework without large fine-tuning. The second issue arises from the presence of a color breaking vacuum which is controlled by Eq. (5.19). For the values of $m_{3 / 2}(\Lambda)$

\footnotetext{
${ }^{2}$ There is considerable freedom on the choice of the scalar masses. The choice made here is more to keep in tune with our procedure for anomaly mediation. As explained above, the exact details of the scalar sector are not very relevant in our parametrization.
} 
considered in this work, it turns out that this limit is always stronger than the upper bound on $C_{\mu}\left(C_{\mu}^{\prime}\right)$ coming from the mass of the Higgs boson. This latter constraint can therefore be ignored. We limit ourselves to the regions of parameter space where Eq. (5.19) is satisfied.

\subsubsection{Current LHC constraints}

To obtain current limits on anomaly and gauge mediation, searches for gluino pair production are recast. In particular, the focus is on searches with either many b-jets, leptons, or large jet-multiplicity. Of course, all of these searches have stringent cuts on missing transverse energy (MET). The chosen searches are summarized in Table 5.1. As a general rule, Ref. [70] dominates over the others. For each of these searches, codes simulating the cuts are implemented. To validate our codes, events were generated with MadGraph 5 [93] intefaced with Pythia 6 [94] and Delphes 3 [95,96]. All four searches were reproduced with good accuracy. There are also constraints coming from electroweakino production for which the experimental bounds found in Refs. [66-68, 71] apply directly. This is because the branching ratios for the charginos and neutralinos that are relevant for our models are the same as the ones used in the simplified models considered in those searches. The bounds are in general much weaker than the one from gluino production and become relevant only in a tiny region of parameter space where the electroweakinos are very light.

Our method to reinterpret the experimental constraints follows closely the procedure of Ref. [97]. Every possible combination of the decay chains given in (5.18) is simulated and the efficiency of every signal region is measured for each of them. The branching fractions are then calculated using the procedure of Section 5.1.3. The gluino pair production cross sections are calculated at NLO+NLL with NLL- 


\begin{tabular}{|c|c|c|c|}
\hline Collaboration & Search & Strategy & Reference \\
\hline ATLAS & JHEP 06 (2014) 035 & $\begin{array}{c}2 \text { same sign / 3 } \\
\text { leptons + 0-3 b-jets }+ \\
\text { MET }\end{array}$ & {$[69]$} \\
\hline ATLAS & JHEP 10 (2014) 024 & $\begin{array}{c}\text { 0-1 leptons }+3 \\
\text { b-jets + MET }\end{array}$ & {$[70]$} \\
\hline CMS & CMS-SUS-13-012 & $\begin{array}{c}\text { High jet-multiplicity } \\
+ \text { MET }\end{array}$ & {$[72]$} \\
\hline CMS & CMS-PAS-SUS-12- & $\begin{array}{c}2 \text { opposite sign } \\
\text { leptons }+ \text { high-jet } \\
\text { multiplicity + 3 b-jets } \\
+ \text { MET }\end{array}$ & {$[3]$} \\
\hline
\end{tabular}

Table 5.1: Gluino pair production searches used to obtain current constraints.

fast [98-102], which we verified using Prospino [103]. The number of expected signal events in a given signal region can then be calculated. The $95 \%$ confidence level signal upper limit can either be read directly from these searches or calculated using the known background and confidence level (CL) techniques [104]. The different signal regions are combined in a boolean fashion [105]. A more thorough approach would require the correlation between the backgrounds of the different signal regions, which is not readily available.

The events are generated with MadGraph 5 [93] interfaced with Pythia 6 [94] and Delphes 3 [95,96]. 10000 events are generated for each grid point. MadGraph generally takes care of decay chains up to the production of the LSP. The only exception is when either $\chi_{2}^{0}$ or $\chi_{1}^{+}$is very close in mass to $\chi_{1}^{0}$. These decays can then be forced to be off-shell and the decay chains become too long to be handled by MadGraph comfortably. In the worst case scenario, $\chi_{2}^{0}$ can decay to $\chi_{1}^{0}$ and an off-shell Higgs which then decays to a $\mathrm{W}$ and a off-shell $\mathrm{W}$ which in turn decays to other particles. To handle these difficult decays, the branching ratios are calculated in advance using the decay functionalities of MadGraph to produce decay tables. $\chi_{2}^{0}$ and $\chi_{1}^{+}$are then 
decayed by Pythia using these results. Delphes handles the detector simulation and is tuned to simulate the ATLAS and CMS detectors.

The results for the 95\% CL limits from ATLAS and CMS are given in Figs. 5.3 and 5.4 for anomaly and gauge mediation respectively. Each one is provided with contour plots of $M_{\tilde{B}}, M_{\tilde{W}}, \mu$, and $\tan \beta$ to relate it to more familiar parameters. The regions forbidden by color breaking vacuum are shown in purple. Overall, gluinos of mass up to $1.3 \mathrm{TeV}$ can be excluded over significant regions of parameter space.

The results for the anomaly mediation spectrum can be easily understood. Over the entire covered parameter space, the gluino decays mainly to charginos. For $C_{\mu}$ between -4 and 4 , the neutral wino is the LSP. The most relevant parameter in this region is then the ratio of the mass of the LSP and of the gluino. Below $C_{\mu}$ equal to 2, this ratio is large and the exclusion limits are strong. Above that value, the mass spectrum becomes compressed and kinematic quantities like MET become much smaller. As such, the exclusion limits drop considerably.

The results for gauge mediation are similar but with a few additional subtleties. Near $C_{\mu}^{\prime}$ equal to -5 , the spectrum is fairly compressed and the wino is too heavy to be produced. The gluino decays softly to $\chi_{1}^{0}$ and quarks, which results in lower constraints. As $C_{\mu}^{\prime}$ increases, the spectrum becomes less compressed and the limits are stronger. However, near $C_{\mu}^{\prime}$ equal to -3 , the winos become light enough to be produced and the gluino decay to chargino dominates. As these decay chains are longer, there is less MET and the constraints are less strong. In a very narrow band around $C_{\mu}^{\prime}$ equal to -1.5 , the wino is the LSP. The chargino then decays softly to a neutral wino. This is similar to gluino decaying to $\chi_{1}^{0}$ and the exclusion reaches the same levels as at $C_{\mu}^{\prime}$ equal to -3 . As $C_{\mu}^{\prime}$ continues to increase, the mass spectrum again becomes compressed to the point where gluinos can only decay to $\chi_{1}^{0}$ and a 
pair of soft bottom quarks and the limits drop considerably. In addition, direct electroweakino production searches from Refs. [66-68, 71] impose limits in a very narrow band near $C_{\mu}^{\prime}$ equal to -2 . This corresponds to when both the wino and bino are light which only occurs around $C_{\mu}^{\prime}$ equal to -2 . This region is shown as a grey band in Fig. 5.4.

\subsubsection{Prospects at LHC 14}

The procedure of the previous section can be modified to predict the discovery and exclusion prospects at the next phase of the LHC. The only differences amount to the signal regions and background estimations.

Two different strategies are adopted to cover the possibilities of the spectrum being compressed or not. When the LSP is considerably lighter than the gluino, kinematic quantities like MET are large and strong kinematic cuts are sufficient to eliminate most of the background. We refer to these signal regions as high MET cuts. On the contrary, when the gluino has a mass close to that of the LSP, quantities like MET become small and the cuts remove most signals. Lowering the cuts does not improve the limits much as the background increases considerably. However, adding the requirement of same sign dileptons (SSDL) drastically cuts the background and allows the kinematic cuts to be made less stringent by exploiting the possible production of leptons during the top decay. The only drawback to SSDL is that a large part of the signal is cut and the resulting limits are less strong than pure high MET cuts in the non-compressed case. The net result is that high MET signal regions usually dominate until the spectrum becomes near degenerate. The exclusion then drops until the signal regions with SSDL become relevant which prevents the exclusion limits from dropping too fast. However, the SSDL cuts eventually also fail 
(a) $M_{\tilde{B}}[\mathrm{GeV}]$

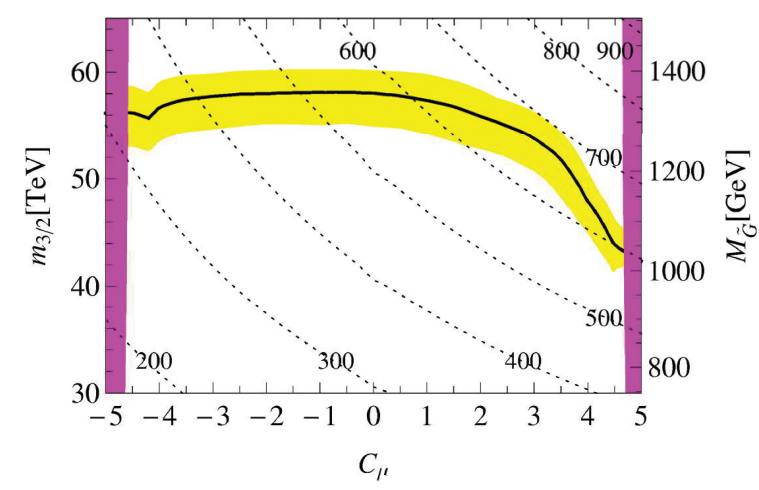

(c) $\mu[\mathrm{TeV}]$

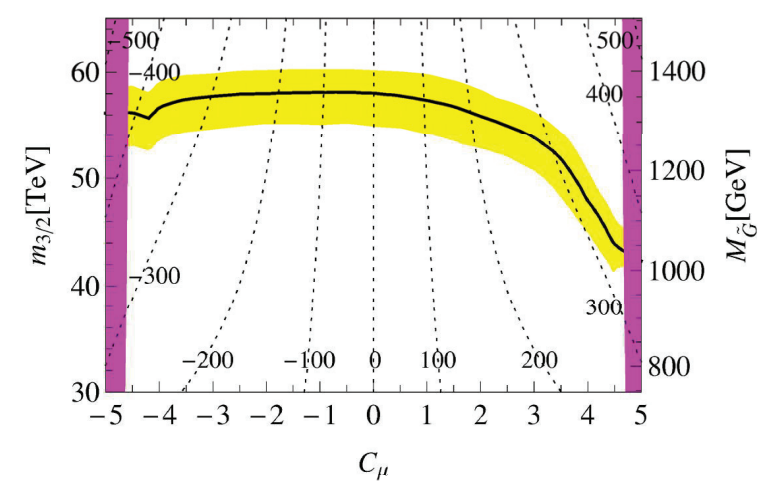

(b) $M_{\tilde{W}}[\mathrm{GeV}]$

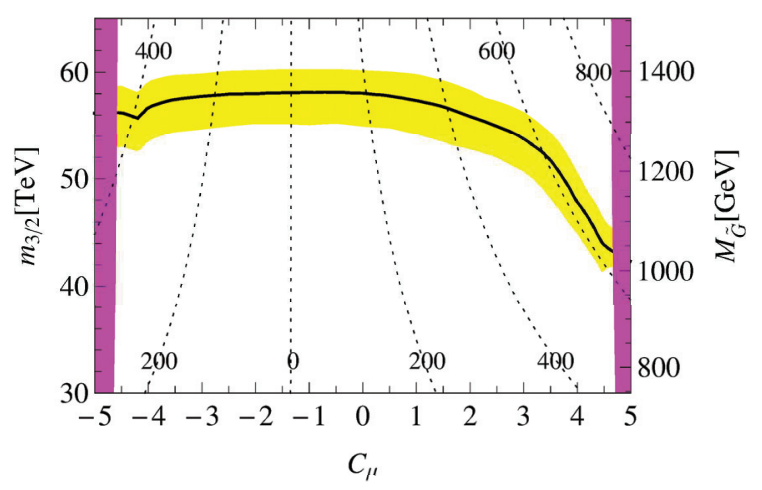

(d) $\tan \beta$

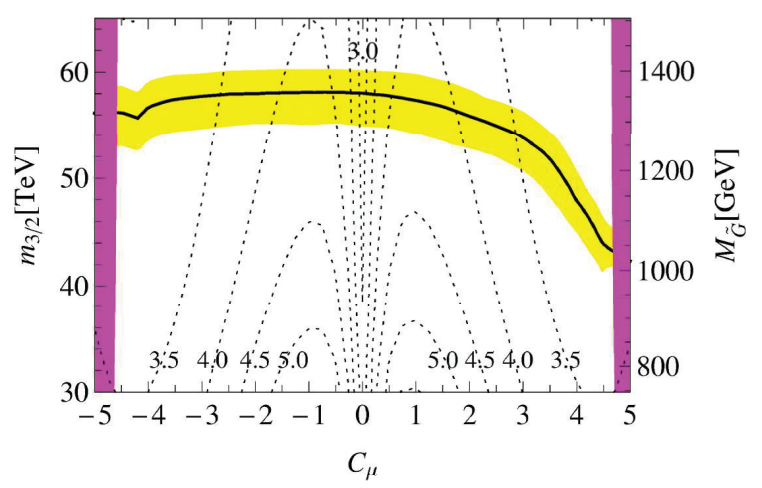

Figure 5.3: 95\% CL exclusion limits for anomaly mediation for Run-I of the LHC. The yellow band corresponds to the $1 \sigma$ uncertainty on the gluino pair production cross section and the purple bands are the forbidden region of color breaking vacuum. Contour lines of constant $M_{\tilde{B}}, M_{\tilde{W}}, \mu$, and $\tan \beta$ are shown respectively in (a), (b), (c), and (d). 
(a) $M_{\tilde{B}}[\mathrm{GeV}]$

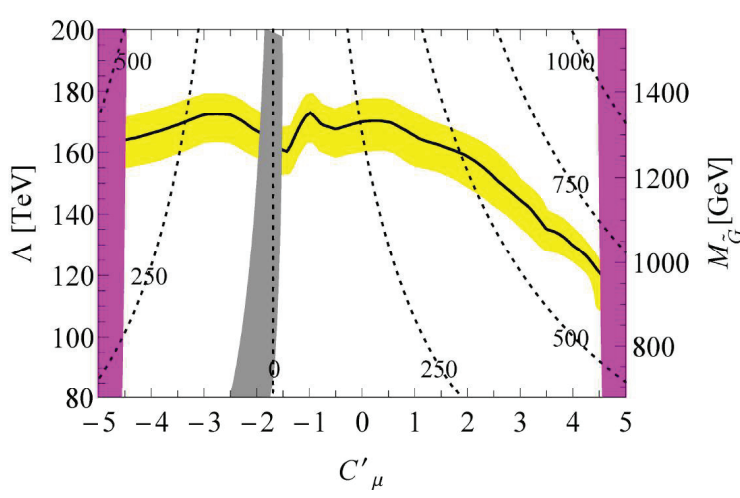

(c) $\mu[\mathrm{TeV}]$

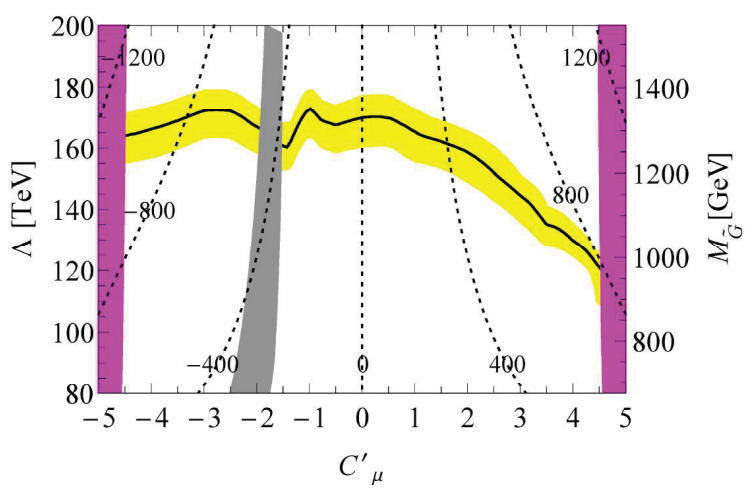

(b) $M_{\tilde{W}}[\mathrm{GeV}]$

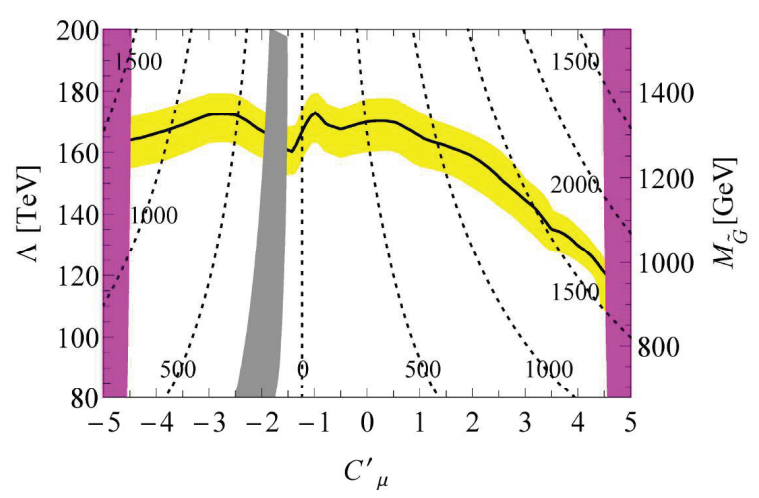

(d) $\tan \beta$

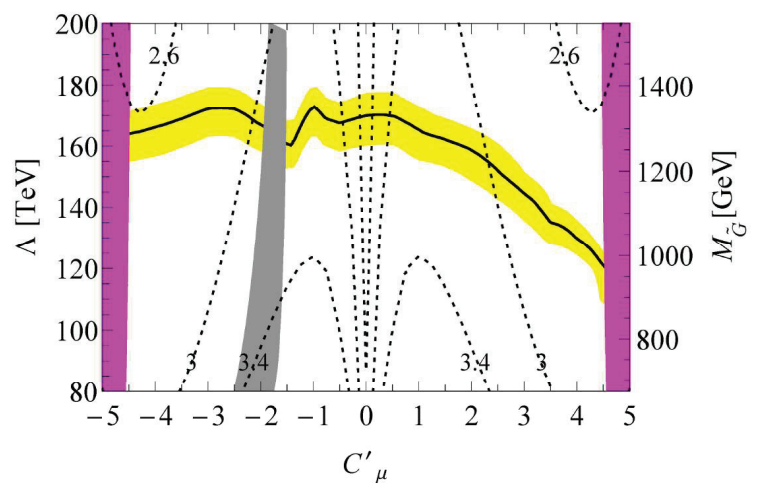

Figure 5.4: 95\% CL exclusion limits for gauge mediation for Run-I of the LHC. The yellow band corresponds to the $1 \sigma$ uncertainty on the gluino pair production cross section and the purple bands are the forbidden region of color breaking vacuum. The grey band corresponds to limits from direct electroweak searches. Contour lines of constant $M_{\tilde{B}}, M_{\tilde{W}}, \mu$, and $\tan \beta$ are shown respectively in (a), (b), (c), and (d). 
when there is not enough phase space for the gluino to produce top quarks.

For the high MET signal regions, the cuts of Ref. [106] for gluino decaying to top quarks and a single lepton are adopted. The cuts for SSDL are taken directly from Ref. [107] and correspond to their gluino-neutralino model with heavy flavour decay for $14 \mathrm{TeV}$. It was verified that both sets of results could be reproduced.

The detector card for Delphes is the standard 14 TeV card from Snowmass [108]. The background estimates for the high MET regions are obtained from the Snowmass online backgrounds [109]. The cuts are simply applied on their events while taking into consideration their relative weight. The Snowmass backgrounds also provide event files with different average number of pile-up vertices. In general, pile-up has very little effect on the high MET regions, while, for SSDL, leptons can possibly get lost in the pile-up jets [107], reducing the efficiency of the signal. This works focuses however on the case of 0 pile-up, as the effect is generally small on most of the parameter space. For high MET cuts, the resulting backgrounds are (23.0, $12.1,2.6,2.1$ ) for the four signal regions of Ref. [106] and $3000 \mathrm{fb}^{-1}$ of integrated luminosity. This can be compared with their result at 140 pile-up vertices of (17.5, $4.8,0.9,1.6)$ and the same integrated luminosity. The backgrounds for SSDL are taken directly from Ref. [107], as we follow very closely their procedure. A $20 \%$ systematic uncertainty on all backgrounds is assumed [107]. The gluino pair production cross section is calculated using NLL-fast [98-102] customized for a $14 \mathrm{TeV}$ collider. The possibility of 300 and $3000 \mathrm{fb}^{-1}$ of integrated luminosity are considered.

The results can be seen for anomaly mediation in Figs. 5.5 and 5.6 for 95\% CL exclusion and $5 \sigma$ discovery respectively, as well as for gauge mediation in Figs. 5.7 and 5.8 for $95 \%$ CL exclusion and $5 \sigma$ discovery respectively. The curves are essentially scaled up versions of the $8 \mathrm{TeV}$ constraints. The anomaly mediation limit curves are 
flatter than those for the current LHC constraints. This can be explained by the fact that the branching ratio to the LSP and two tops decreases more slowly as $C_{\mu}$ increases because heavier gluinos are being probed.

\subsubsection{Prospects at a $100 \mathrm{TeV}$ collider}

To fully explore the possibility of discovering Mini-Split Supersymmetry at colliders, the prospects at a $100 \mathrm{TeV}$ collider are studied following the same procedure as in the previous two sections. The high MET cuts are adapted from Ref. [81], which are themselves based on Ref. [110]. These cuts rely on $M_{\text {eff }}$ which is defined as

$$
M_{\mathrm{eff}}=\sum_{i} p_{T}(i)+\mathrm{MET} .
$$

The sum is on jets with $p_{T}>50 \mathrm{GeV}$ and $|\eta|<5$ and leptons with $p_{T}>15 \mathrm{GeV}$ and $|\eta|<2.5$. The analysis is pushed further than that of Ref. [81] by requiring b-jets, implementing detector simulations, and using a set of signal regions optimized for different regions of parameter space. The preselection cuts are given by [81]

- Lepton veto,

- At least two jets with $p_{T}>0.1 M_{\text {eff }}$,

- $\mathrm{MET}>0.2 M_{\mathrm{eff}}$,

- $p_{T}\left(j_{1}\right)<0.35 M_{\mathrm{eff}}$,

- $\Delta \phi\left(j_{1}, \mathrm{MET}\right)<\pi-0.2$,

- $\Delta \phi\left(j_{1}, j_{2}\right)<2 \pi / 3$. 
(a) $M_{\tilde{B}}[\mathrm{TeV}]$

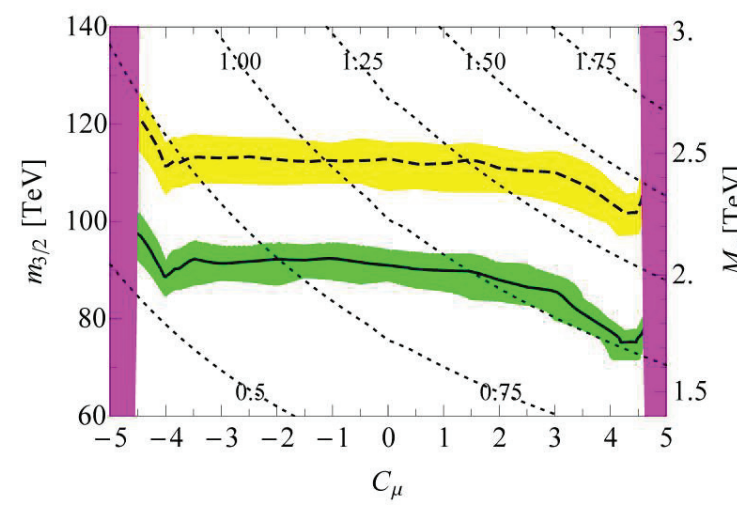

(c) $\mu[\mathrm{PeV}]$

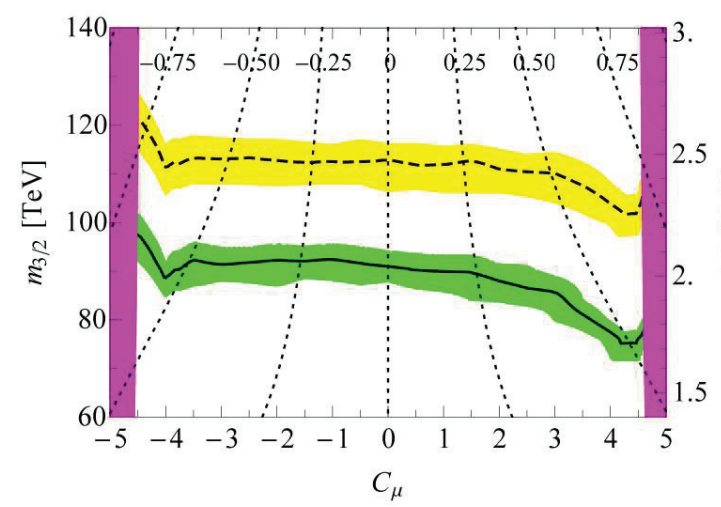

(b) $M_{\tilde{W}}[\mathrm{TeV}]$

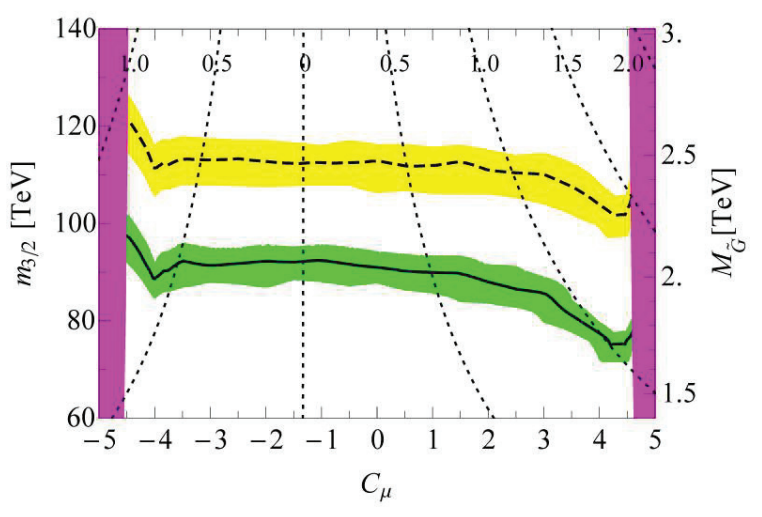

(d) $\tan \beta$

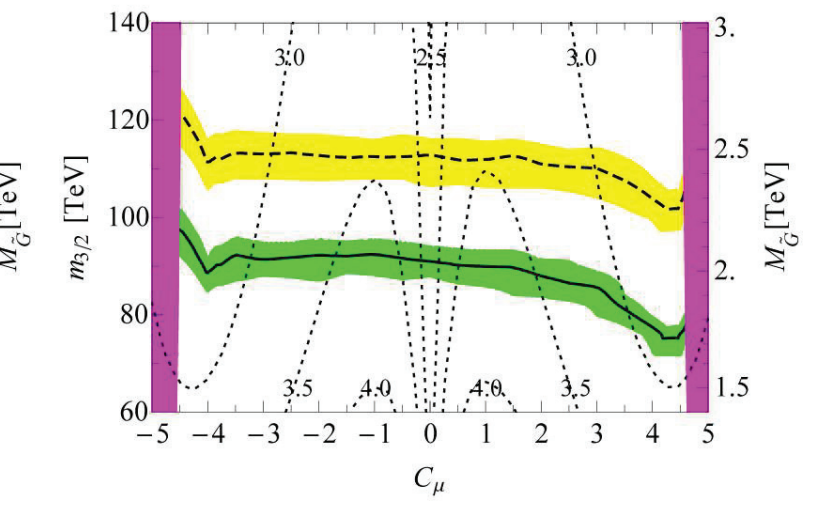

Figure 5.5: 95\% CL exclusion limits for anomaly mediation at LHC 14 for (solid) $300 \mathrm{fb}^{-1}$ and (dashed) $3000 \mathrm{fb}^{-1}$ integrated luminosity. The green band corresponds to the $1 \sigma$ uncertainty on the gluino pair production cross section for $300 \mathrm{fb}^{-1}$, the yellow band corresponds to the $1 \sigma$ uncertainty on the gluino pair production cross section for $3000 \mathrm{fb}^{-1}$, and the purple bands are the forbidden region of color breaking vacuum. Contour lines of constant $M_{\tilde{B}}, M_{\tilde{W}}, \mu$, and $\tan \beta$ are shown respectively in (a), (b), (c), and (d). 
(a) $M_{\tilde{B}}[\mathrm{TeV}]$

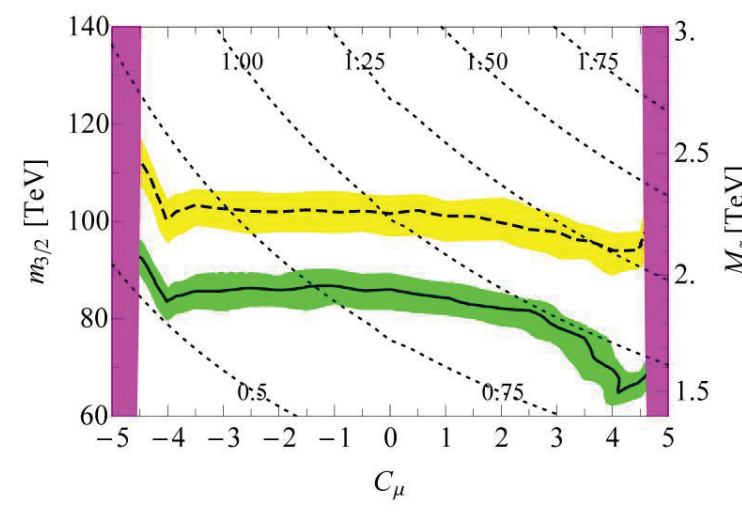

(c) $\mu[\mathrm{PeV}]$

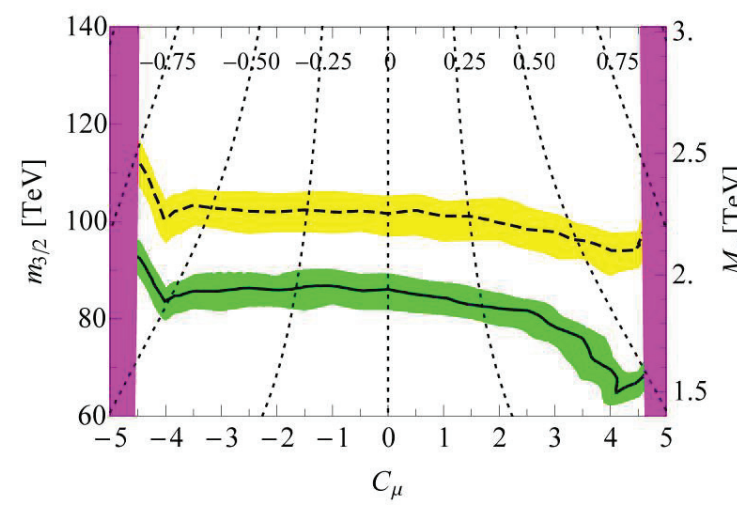

(b) $M_{\tilde{W}}[\mathrm{TeV}]$

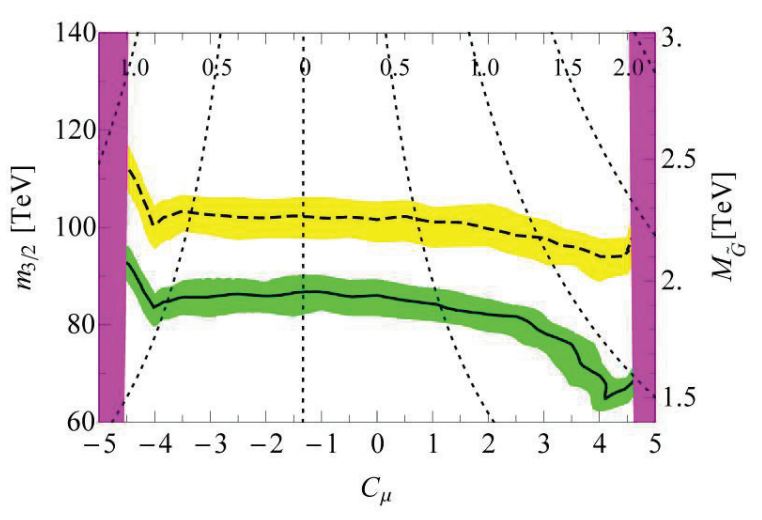

(d) $\tan \beta$

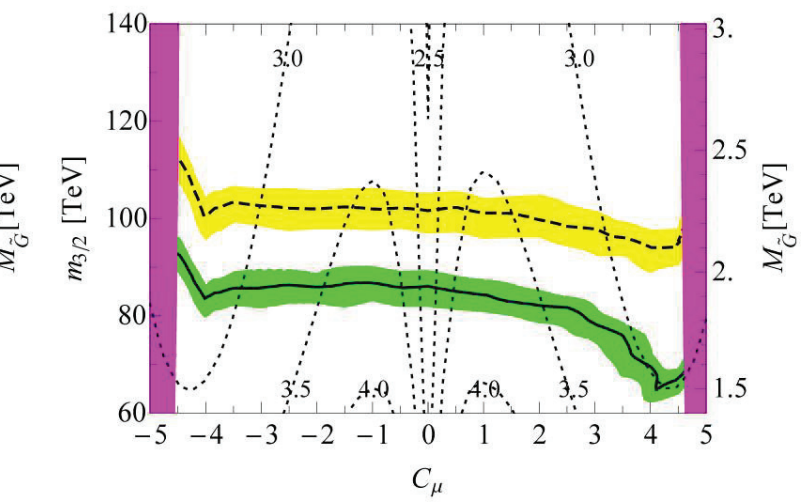

Figure 5.6: $5 \sigma$ discovery limits for anomaly mediation at LHC 14 for (solid) $300 \mathrm{fb}^{-1}$ and (dashed) $3000 \mathrm{fb}^{-1}$ integrated luminosity. The green band corresponds to the $1 \sigma$ uncertainty on the gluino pair production cross section for $300 \mathrm{fb}^{-1}$, the yellow band corresponds to the $1 \sigma$ uncertainty on the gluino pair production cross section for $3000 \mathrm{fb}^{-1}$, and the purple bands are the forbidden region of color breaking vacuum. Contour lines of constant $M_{\tilde{B}}, M_{\tilde{W}}, \mu$, and $\tan \beta$ are shown respectively in (a), (b), (c), and (d). 
(a) $M_{\tilde{B}}[\mathrm{TeV}]$

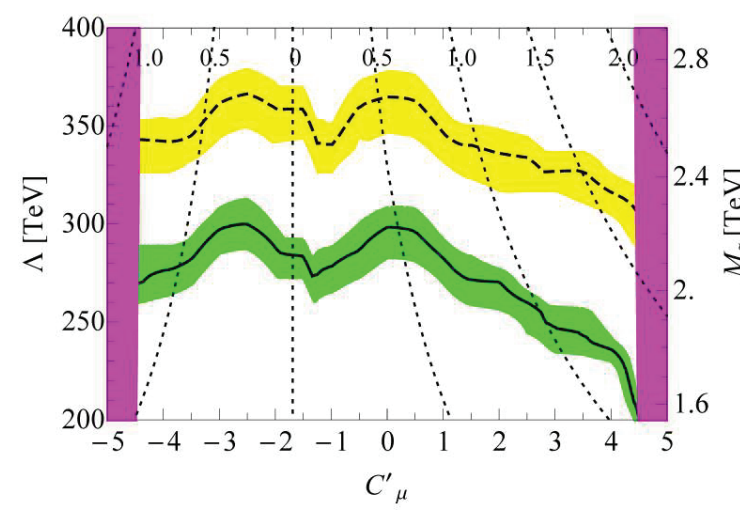

(c) $\mu[\mathrm{PeV}]$

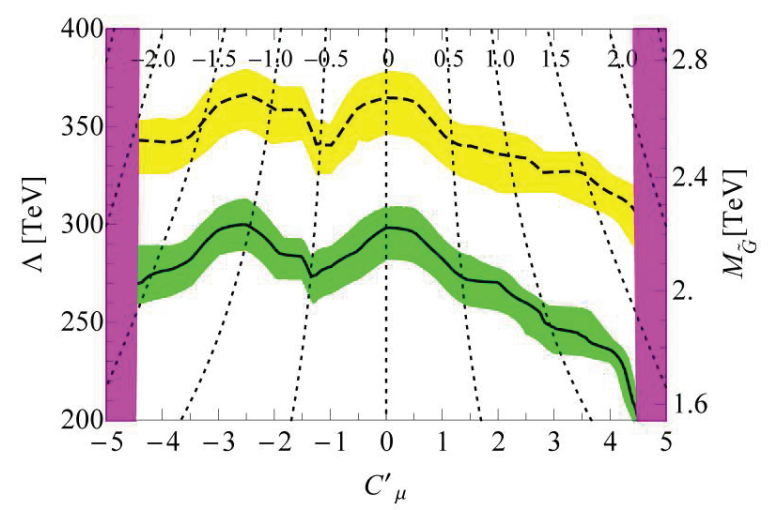

(b) $M_{\tilde{W}}[\mathrm{TeV}]$

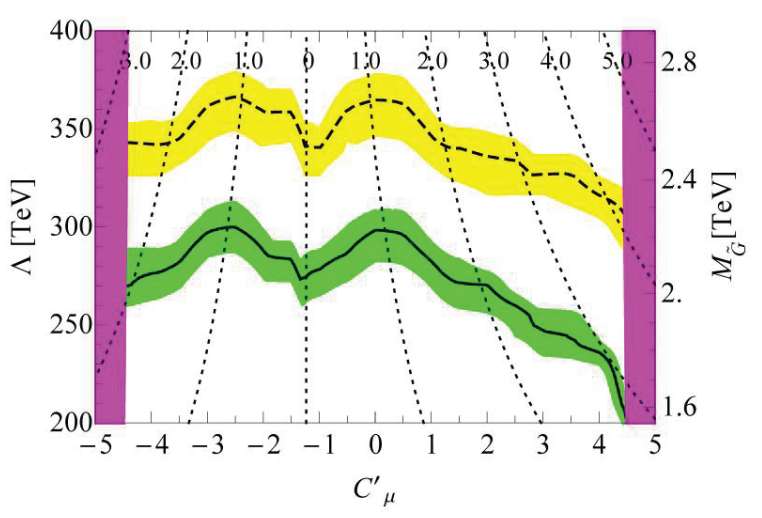

(d) $\tan \beta$

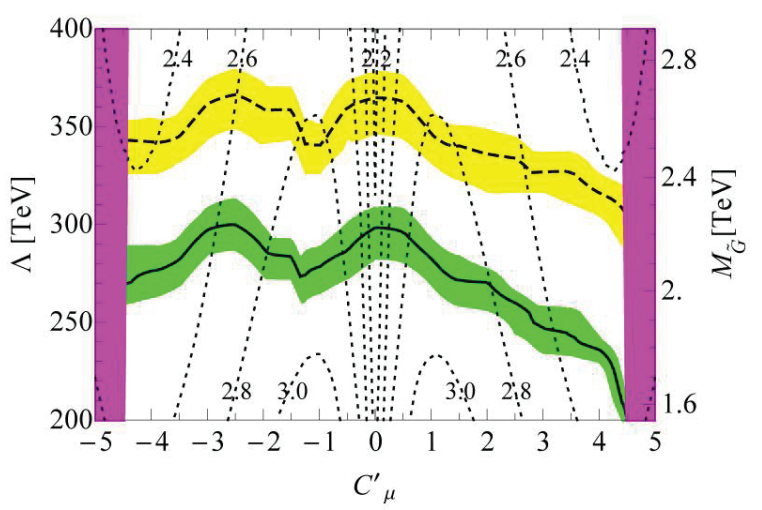

Figure 5.7: 95\% CL exclusion limits for gauge mediation at LHC 14 for (solid) 300 $\mathrm{fb}^{-1}$ and (dashed) $3000 \mathrm{fb}^{-1}$ integrated luminosity. The green band corresponds to the $1 \sigma$ uncertainty on the gluino pair production cross section for $300 \mathrm{fb}^{-1}$, the yellow band corresponds to the $1 \sigma$ uncertainty on the gluino pair production cross section for $3000 \mathrm{fb}^{-1}$, and the purple bands are the forbidden region of color breaking vacuum. Contour lines of constant $M_{\tilde{B}}, M_{\tilde{W}}, \mu$, and $\tan \beta$ are shown respectively in (a), (b), (c), and (d). 
(a) $M_{\tilde{B}}[\mathrm{TeV}]$

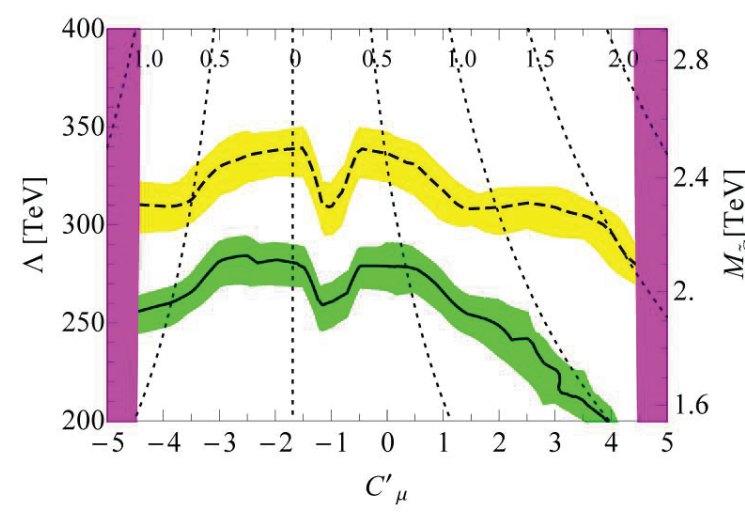

(c) $\mu[\mathrm{PeV}]$

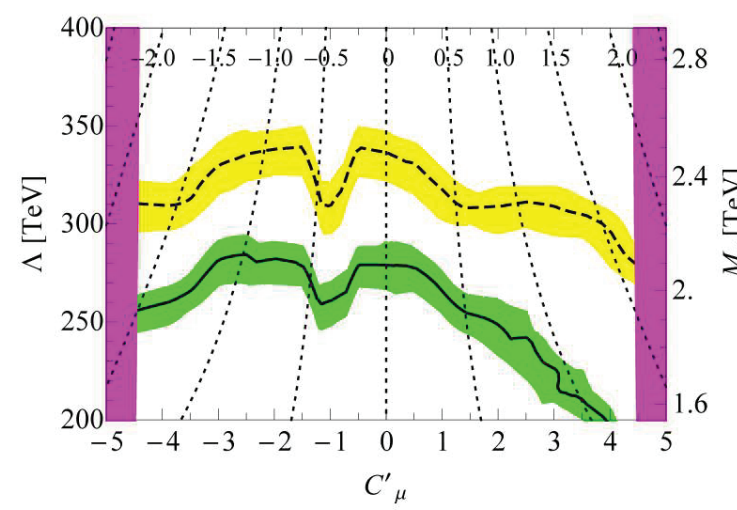

(b) $M_{\tilde{W}}[\mathrm{TeV}]$

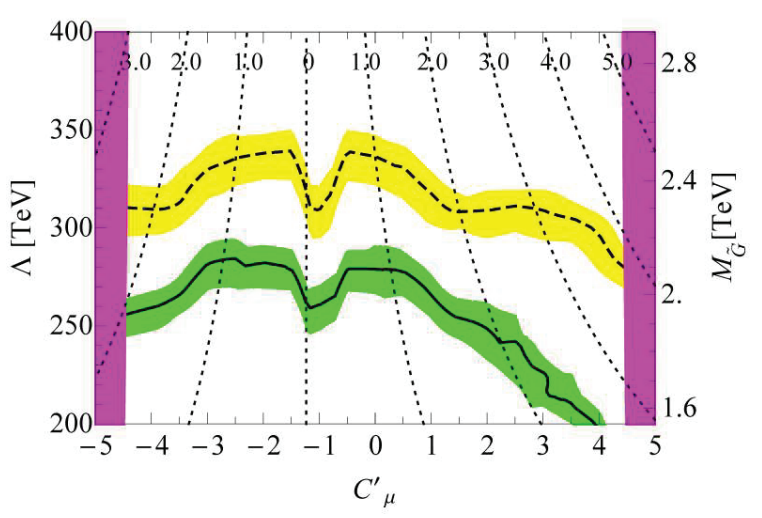

(d) $\tan \beta$

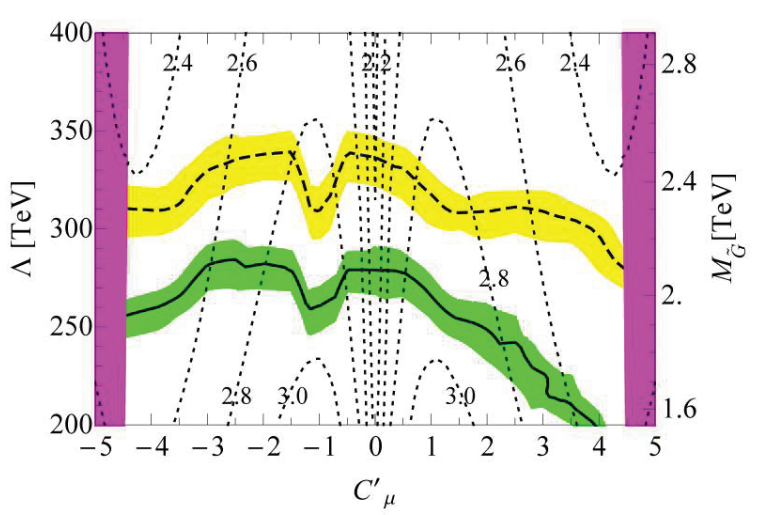

Figure 5.8: $5 \sigma$ discovery limits for gauge mediation at LHC 14 for (solid) $300 \mathrm{fb}^{-1}$ and (dashed) $3000 \mathrm{fb}^{-1}$ integrated luminosity. The green band corresponds to the $1 \sigma$ uncertainty on the gluino pair production cross section for $300 \mathrm{fb}^{-1}$, the yellow band corresponds to the $1 \sigma$ uncertainty on the gluino pair production cross section for $3000 \mathrm{fb}^{-1}$, and the purple bands are the forbidden region of color breaking vacuum. Contour lines of constant $M_{\tilde{B}}, M_{\tilde{W}}, \mu$, and $\tan \beta$ are shown respectively in (a), (b), (c), and (d). 


\begin{tabular}{|c|c|c|c|}
\hline SR & b-jets & $M_{\text {eff }}[\mathrm{TeV}]$ & Background \\
\hline hMETb3A & $\geq 3$ & $>15.0$ & 23.4 \\
\hline hMETb3B & $\geq 3$ & $>17.5$ & 7.8 \\
\hline hMETb3C & $\geq 3$ & $>20.0$ & 2.3 \\
\hline hMETb4A & $\geq 4$ & $>12.5$ & 12.6 \\
\hline hMETb4B & $\geq 4$ & $>15.0$ & 3.8 \\
\hline hMETb4C & $\geq 4$ & $>17.5$ & 1.5 \\
\hline hMETb4D & $\geq 4$ & $>20.0$ & 0.5 \\
\hline
\end{tabular}

Table 5.2: Signal regions for high MET. The background for $3 \mathrm{ab}^{-1}$ is also included.

The different signal regions correspond to different combinations of minimum b-jets requirements and $M_{\text {eff }}$ cuts and are given in Table 5.2.

The SSDL cuts and the corresponding backgrounds are taken directly from Ref. [107] and correspond to their search for a gluino-neutralino model with heavy flavour decays. It was verified that the code reproduces their results.

The detector card for Delphes is the standard $100 \mathrm{TeV}$ card from Snowmass [108]. The background estimates for high MET are again obtained from the Snowmass online backgrounds [109]. The backgrounds for the high MET signal regions are shown in Table 5.2 for $3 \mathrm{ab}^{-1}$ integrated luminosity. A $20 \%$ systematic uncertainty on all backgrounds is assumed [107]. The discussion of pile-up for high MET or SSDL from the previous section still holds. The case of 0 pile-up is assumed, as the average pileup of a future $100 \mathrm{TeV}$ collider is still unknown and as it only has a non-negligible effect on a small portion of our parameter space. The gluino pair production cross section is calculated using NLL-fast [98-102] customized for a $100 \mathrm{TeV}$ collider.

The results are again scaled up versions of LHC constraints with possible exclusion of up to a $14 \mathrm{TeV}$ gluino in a large region of parameter space and discovery of up to $12 \mathrm{TeV}$. These numbers are similar to those obtained by Ref. [81] which 
seem somewhat more optimistic (with a possible discovery of up to $\sim 15 \mathrm{TeV}$ ). ${ }^{3}$ For anomaly mediation, exclusion limits are governed by high MET signal regions and are thus very high until $C_{\mu}$ reaches 1 . At this point, the spectrum becomes compressed and the limits drop. The SSDL bins then dominate and the limits stabilize with a discovery reach of about $7 \mathrm{TeV}$ (this number is in fact quite close to the result of Ref. [107]). The exact same thing happens in the case of gauge mediation, except that the limits drop at $C_{\mu}^{\prime}$ equal to 0 .

\subsection{Additional comments}

To conclude this chapter, we summarize the prospects for Mini-Split Supersymmetry at different colliders. The gluino is currently constrained by Run-I of the LHC to be above $1.3 \mathrm{TeV}$ in the best case scenario, while LHC 14 and a $100 \mathrm{TeV}$ collider could potentially push this limit to around $2.7 \mathrm{TeV}$ and $14 \mathrm{TeV}$ respectively. LHC 14 could potentially discover a gluino as heavy as $2.5 \mathrm{TeV}$, while a future $100 \mathrm{TeV}$ collider could discover one up to $12 \mathrm{TeV}$.

While the goal of this work was to explore the collider phenomenology of MiniSplit models, dark matter properties could also be used to further restrict the parameter space. The thermal abundance of the dark matter candidate is strongly dependent on the identity of the LSP. For a wino LSP, the correct thermal relic abundance can be obtained for a wino mass of $2.7 \mathrm{TeV}[65,74]$. This region of parameter space is not constrained by the LHC, but is within reach of a $100 \mathrm{TeV}$ collider. Wino LSP with lighter mass could be accommodated by invoking non-thermal production [74]. Similarly, bino LSP, which tend to overclose the universe, could be accommodated if there was late entropy production or a low reheating temperature.

\footnotetext{
${ }^{3}$ This might be due, for example, to the fact that we have used a detector simulation, but we haven't directly checked that hypothesis.
} 
(a) $M_{\tilde{B}}[\mathrm{TeV}]$

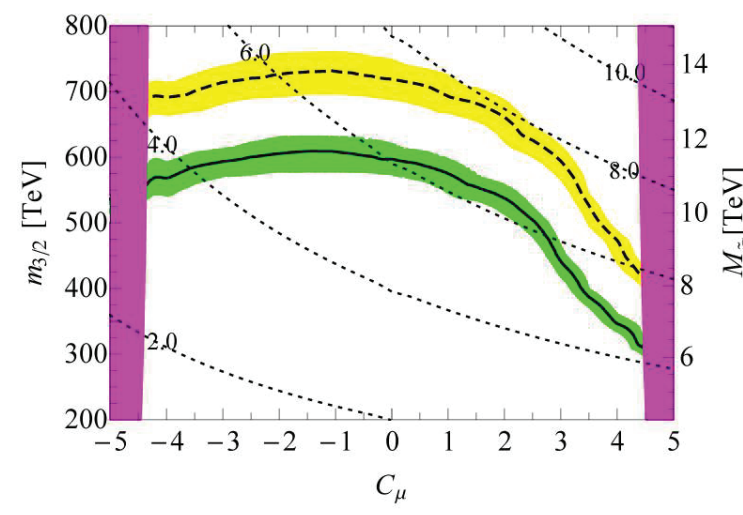

(c) $\mu[\mathrm{PeV}]$

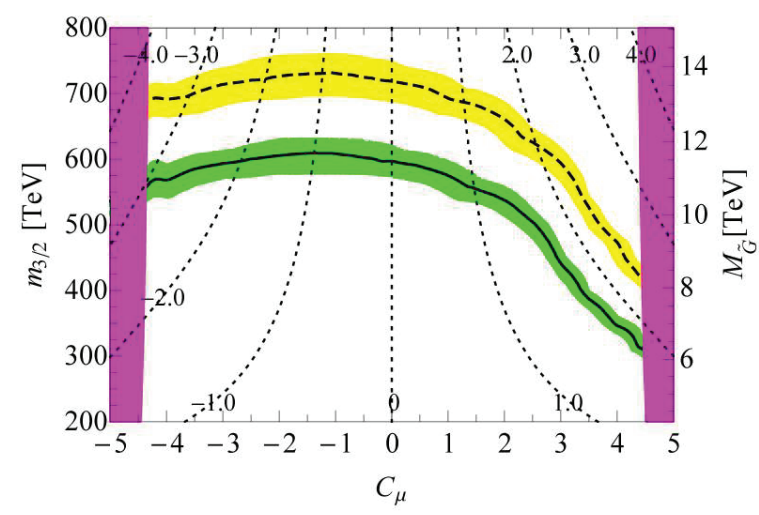

(b) $M_{\tilde{W}}[\mathrm{TeV}]$

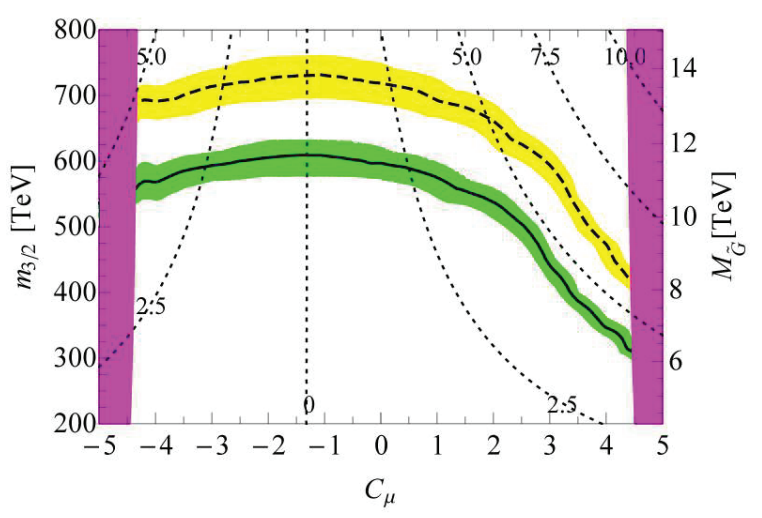

(d) $\tan \beta$

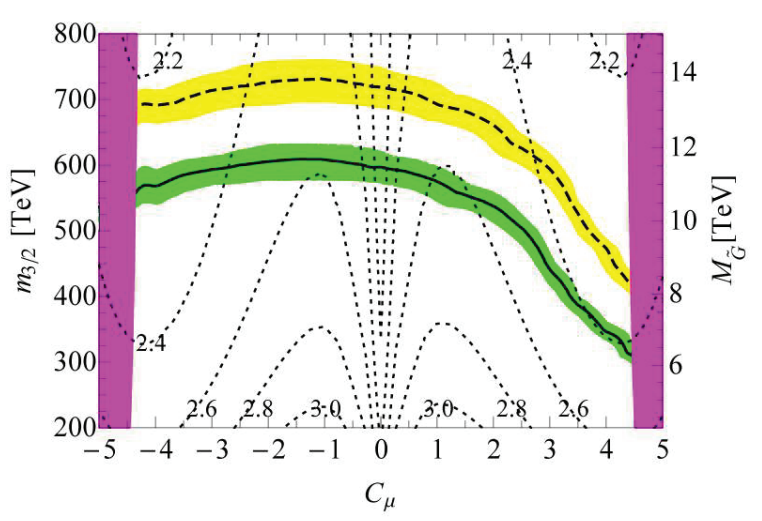

Figure 5.9: 95\% CL (dashed) exclusion and $5 \sigma$ (solid) discovery limits for anomaly mediation at a $100 \mathrm{TeV} p p$ collider with $3 \mathrm{ab}^{-1}$ integrated luminosity. The yellow band corresponds to the $1 \sigma$ uncertainty on the gluino pair production cross section for $95 \%$ exclusion, the green band corresponds to the $1 \sigma$ uncertainty on the gluino pair production cross section for $5 \sigma$ discovery, and the purple bands are the forbidden region of color breaking vacuum. Contour lines of constant $M_{\tilde{B}}, M_{\tilde{W}}, \mu$, and $\tan \beta$ are shown respectively in (a), (b), (c), and (d). 
(a) $M_{\tilde{B}}[\mathrm{TeV}]$

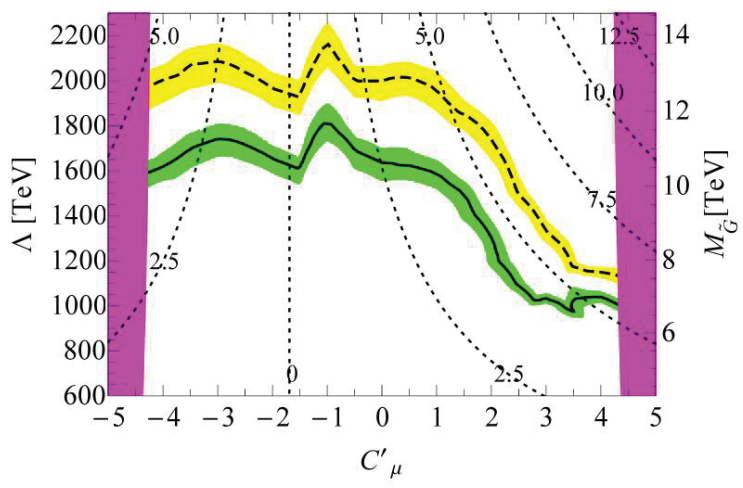

(c) $\mu[\mathrm{PeV}]$

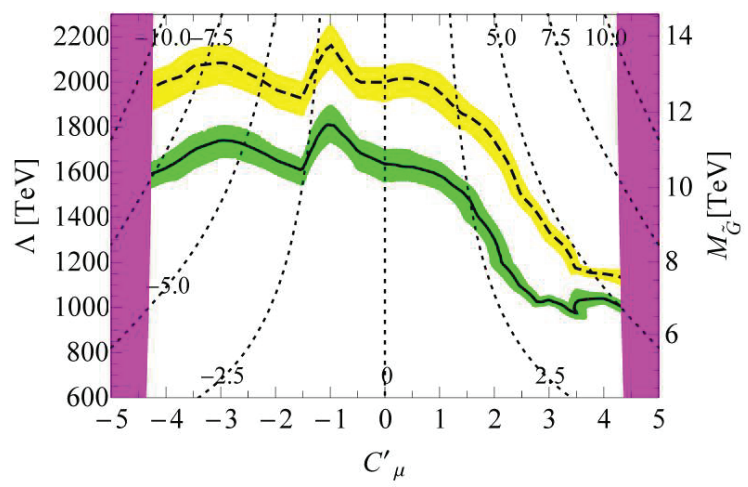

(b) $M_{\tilde{W}}[\mathrm{TeV}]$

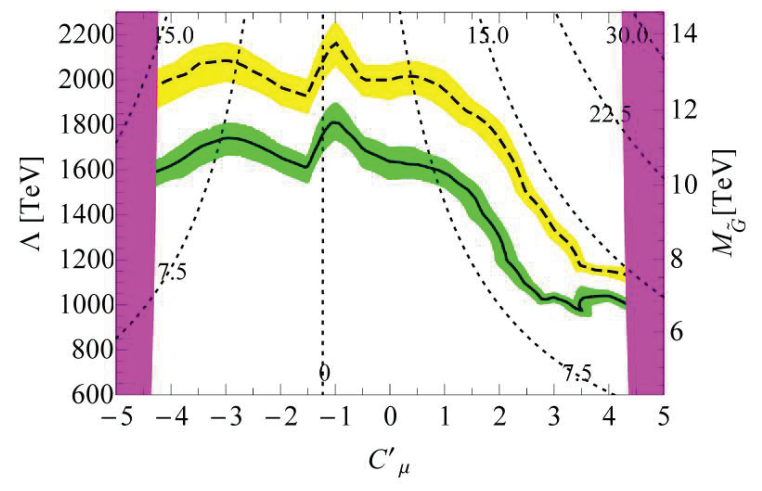

(d) $\tan \beta$

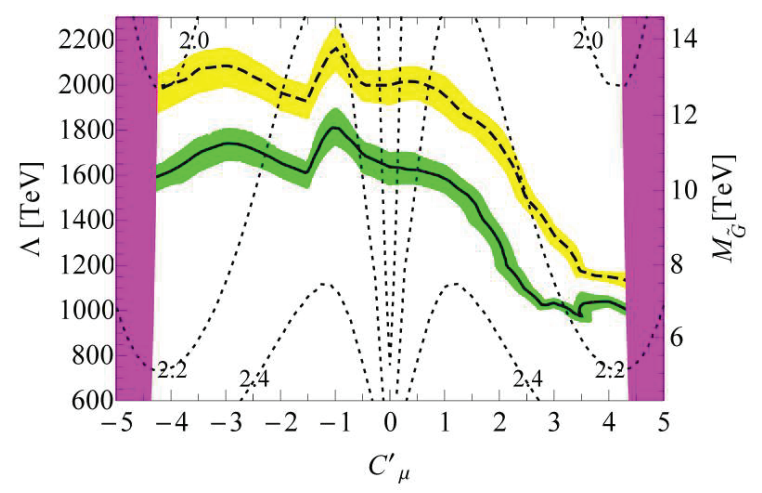

Figure 5.10: 95\% CL (dashed) exclusion and 5 $\sigma$ (solid) discovery limits for gauge mediation at a $100 \mathrm{TeV} p p$ collider with $3 \mathrm{ab}^{-1}$ integrated luminosity. The yellow band corresponds to the $1 \sigma$ uncertainty on the gluino pair production cross section for $95 \%$ exclusion, the green band corresponds to the $1 \sigma$ uncertainty on the gluino pair production cross section for $5 \sigma$ discovery, and the purple bands are the forbidden region of color breaking vacuum. Contour lines of constant $M_{\tilde{B}}, M_{\tilde{W}}, \mu$, and $\tan \beta$ are shown respectively in (a), (b), (c), and (d). 
Finally, we mention that, while outside the scope of this chapter, there could be additional constraints from disappearing tracks. See Ref. [111] for an example. Such constraints can become important in the wino LSP case if the mass splitting between the charged and neutral gauginos is smaller than the mass of a charged pion. The limits from gluino pair production are still expected to dominate. 


\section{Chapter 6}

\section{Models of $U(1)_{R}$ Supersymmetry}

As experimental constraints on Supersymmetry continue to improve, alternatives to the MSSM that might be less minimal but otherwise more natural are becoming increasingly attractive. A notable possibility is to have Dirac gauginos instead of Majorana ones (see Refs. [58,112-127]). One of the main advantages of this approach is that it allows $R$-parity to be promoted to a continuous $U(1)_{R}$ symmetry. Chapters 7 and 8 are devoted to the phenomenological implications of two models of this type. To avoid redundancy between these two chapters and to provide an adequate background, we devote this chapter to a short introduction to general models with $U(1)_{R}$ symmetry.

This chapter is organized as follow. First, the process by which Dirac gauginos can be generated is explained. The inclusion of a $U(1)_{R}$ symmetry is then discussed. Models in which the $U(1)_{R}$ symmetry can be associated with lepton and baryon numbers are finally described. This chapter follows closely Ref. [128]. 


\subsection{Dirac gauginos}

The first step in obtaining Dirac masses for gauginos is to extend the superfield content. The chiral superfields

$$
G, T, S
$$

are introduced, where $G$ is an adjoint of color, $T$ an adjoint of $S U(2)_{L}$ and $S$ a singlet. Dirac masses are then generated via terms of the form (shown here for the winos)

$$
-\int d^{2} \theta \frac{\mathcal{W}^{\alpha} \mathcal{W}_{2 \alpha}^{a} T^{a}}{\sqrt{2} M}+\text { h.c. }
$$

where $\mathcal{W}_{2}^{a}$ is the $S U(2)$ superfield strength and $\mathcal{W}^{\prime}$ is a real superfield that acts as a spurion with a non-zero $D$ term: $\mathcal{W}_{\alpha}^{\prime}=-D^{\prime} \theta_{\alpha}+\cdots$. This leads to a mass term of the form

$$
-M_{2}^{D} \tilde{T}^{a} \cdot \tilde{W}^{a}+\text { h.c. }
$$

where $\tilde{W}^{a}$ is the usual gaugino and $\tilde{T}^{a}$ the fermion component of $T^{a}$. The term (6.2) also leads to a non-standard expression for the auxiliary fields $D_{i}$,

$$
\begin{aligned}
& D_{1}=g^{\prime} \sum_{\Phi} Y_{\Phi} \Phi^{\dagger} \Phi+\sqrt{2}\left(M_{1}^{D} S+\text { h.c. }\right) \\
& D_{2}^{a}=g \sum_{\Phi} \Phi^{\dagger} t^{a} \Phi+\sqrt{2}\left(M_{2}^{D} T^{a}+\text { h.c. }\right) \\
& D_{3}^{a}=g_{s} \sum_{\Phi} \Phi^{\dagger} t_{c}^{a} \Phi+\sqrt{2}\left(M_{3}^{D} O^{a}+\text { h.c. }\right),
\end{aligned}
$$

where the $t^{a}$ 's and $t_{c}^{a}$ 's are respectively the generators of $S U(2)_{L}$ and $S U(3)_{c}$ associated with the representation under which the field $\Phi$ transforms and $Y_{\Phi}$ its hypercharge. 


\begin{tabular}{|c|cccccccccccc|}
\hline & $Q_{i}$ & $U_{i}$ & $D_{i}$ & $L_{i}$ & $E_{i}$ & $H_{u}$ & $H_{d}$ & $R_{u}$ & $R_{d}$ & $S$ & $T$ & $O$ \\
\hline$U(1)_{R_{0}}$ & 1 & 1 & 1 & 1 & 1 & 0 & 0 & 2 & 2 & 0 & 0 & 0 \\
$U(1)_{L}$ & 0 & 0 & 0 & 1 & -1 & 0 & 0 & 0 & 0 & 0 & 0 & 0 \\
$U(1)_{B}$ & $1 / 3$ & $-1 / 3$ & $-1 / 3$ & 1 & 1 & 0 & 0 & 0 & 0 & 0 & 0 & 0 \\
\hline
\end{tabular}

Table 6.1: Superfields and their $U(1)$ charges.

\section{$6.2 U(1)_{R}$ symmetries}

Having Dirac gauginos instead of Majorana ones has several advantages. Some of them are that the cross section for squark pair production is reduced [129], thereby loosening their constraints, and that the mass corrections to squarks are finite [58]. Several flavour constraints are also relaxed [116]. Perhaps more importantly, Dirac gauginos allow for $U(1)_{R}$ symmetries, which would otherwise be broken by gaugino Majorana masses.

The $U(1)_{R}$ symmetry is analogous to $R$-parity in the sense that Grassmann coordinates have a $U(1)_{R}$ charge and that therefore the different components of a multiplet are charged differently under this group. We assign a charge of -1 to $\theta$. Two additional $S U(2)_{L}$ doublet chiral superfields are introduced to form mass terms for the Higgsinos [116]. They are labeled $R_{u}$ and $R_{d}$ and have a hypercharge of $+1 / 2$ and $-1 / 2$ respectively. A possible assignment of $U(1)_{R}$ charges referred to as $U(1)_{R_{0}}$ is shown in Table 6.1. Lepton and baryon numbers are also shown.

The following superpotential is consistent with the $U(1)_{R_{0}}$ charge assignment,

$$
W_{0}=y_{i j}^{u} H_{u} Q_{i} U_{j}+\tilde{y}_{i j}^{d} H_{d} Q_{i} D_{j}+\tilde{y}_{i j}^{e} H_{d} L_{i} E_{j}+\mu_{u} H_{u} R_{d}+\mu_{d} R_{u} H_{d}
$$




\begin{tabular}{|c|cccccccccccc|}
\hline & $Q_{i}$ & $U_{i}$ & $D_{i}$ & $L_{i}$ & $E_{i}$ & $H_{u}$ & $H_{d}$ & $R_{u}$ & $R_{d}$ & $S$ & $T$ & $O$ \\
\hline$U(1)_{R_{1}=R_{0}-L}$ & 1 & 1 & 1 & 0 & 2 & 0 & 0 & 2 & 2 & 0 & 0 & 0 \\
$U(1)_{R_{2}=R_{0}+B}$ & $4 / 3$ & $2 / 3$ & $2 / 3$ & 1 & 1 & 0 & 0 & 2 & 2 & 0 & 0 & 0 \\
$U(1)_{R_{3}=R_{0}+L}$ & 1 & 1 & 1 & 2 & 0 & 0 & 0 & 2 & 2 & 0 & 0 & 0 \\
\hline
\end{tabular}

Table 6.2: Superfields and their $U(1)_{R}$ charges for three alternative models.

In addition, the following superpotential is also allowed,

$$
W_{\text {adj }}=S\left(\lambda_{u}^{S} H_{u} R_{d}+\lambda_{d}^{S} R_{u} H_{d}\right)+\left(\lambda_{u}^{T} H_{u} T R_{d}+\lambda_{d}^{T} R_{u} T H_{d}\right)
$$

where $T$ is a shorthand notation for $T^{a} \sigma^{a} / 2$. The symmetries of Table 6.1 do not allow for any of the operators that break the usual $R$-parity of the MSSM, i.e. $L H_{u}, L L E$, $L Q D$ and $U D D$. The breaking of $R$-parity is usually known as $R$-parity violation $(\mathrm{RPV})$. It is however possible to allow for such operators by taking a new $U(1)_{R}$ charge assignment that is a linear combination of $U(1)_{R_{0}}, U(1)_{B}$ and $U(1)_{L}$. Table 6.2 gives a few examples. Under these symmetries, the following superpotentials are allowed in addition to those of Eqs. (6.5) and (6.6)

$$
\begin{array}{ll}
W_{1}=\frac{\lambda_{i j k}}{2} L_{i} L_{j} E_{k}+\lambda_{i j k}^{\prime} L_{i} Q_{j} D_{k} & \left(R_{1}=R_{0}-L\right), \\
W_{2}=\frac{\lambda_{i j k}^{\prime \prime}}{2} U_{i} D_{j} D_{k} & \left(R_{2}=R_{0}+B\right), \\
W_{3}=\mu_{L}^{i} H_{u} L_{i} & \left(R_{3}=R_{0}+L\right) .
\end{array}
$$

It is in principle possible to add a term $\mu_{L}^{i} R_{u} L_{i}$ to $W_{1}$. However, it can be rotated away with an $S U(4)$ field redefinition [24]. To complete the theory, it is necessary to 
specify the soft terms. For all $R_{i}$ 's, the following soft terms are allowed

$$
\begin{aligned}
\mathcal{V}_{0}^{\mathrm{soft}}=\sum_{i} \Phi_{i}^{\dagger} \Phi_{i}+ & {\left[t_{S} S+\frac{1}{2} b_{S} S^{2}+\frac{1}{3} A_{S} S^{3}+\frac{1}{2} B_{T} T^{2}+\frac{1}{2} b_{O} O^{2}+B_{\mu} H_{u} H_{d}\right.} \\
& \left.+A_{S} S H_{u} H_{d}+A_{T} H_{u} T H_{d}+A_{T}^{\lambda} S T^{2}+A_{O}^{\lambda} S O^{2}+\text { h.c }\right]
\end{aligned}
$$

where the sum runs over every scalar, the scalar components are denoted by the same symbol as their superfield, $T^{2}$ is a short notation for $T^{a} T^{a}$ and similarly for $O^{2}$. In addition, $R_{1}$ allows for the additional soft terms

$$
\mathcal{L}_{1}^{\mathrm{soft}}=B_{\mu_{L}}^{i} H_{u} L_{i}+A_{S}^{i} S H_{u} L_{i}+A_{T}^{i} H_{u} T L_{i}
$$

The models with $R_{2}$ and $R_{3}$ only allow for the soft terms of Eq. (6.8).

\subsection{1 $U(1)_{R_{1}}$ symmetry}

Models with $R=R_{1}$ are studied more carefully in Chapter 7 . In this scenario, the sneutrinos will in general acquire a vev. It is possible to have only one of the sneutrinos acquire one via an $S U(4)$ field redefinition [128]. The $L_{i}$ which does so is then labeled $L_{a}$. It provides a mass to the charged leptons and the down-type quarks, essentially playing the role of $H_{d}$ in the MSSM. The superfield $H_{d}$ is therefore not necessary anymore. The mass $\mu_{d}$ is then assumed very large and $H_{d}$ and $R_{u}$ are integrated out. The two other leptons are referred to as $L_{b}$ and $L_{c}$.

Under these assumptions, the superpotential can be rewritten as

$$
W=\mu H_{u} R_{d}+\lambda_{S} S H_{u} R_{d}+\lambda_{T} H_{u} T R_{d}+y_{i j}^{u} H_{u} Q_{i} U_{j}-y_{j k}^{e} L_{a} L_{j} E_{k}-y_{j k}^{d} L_{a} Q_{j} D_{k}
$$


where we dropped indices that are now irrelevant. The soft terms become

$$
\begin{aligned}
\mathcal{V}_{\text {soft }}=\sum_{i} \Phi_{i}^{\dagger} \Phi_{i}+ & {\left[t_{S} S+\frac{1}{2} b_{S} S^{2}+\frac{1}{2} B_{T} T^{2}+\frac{1}{2} b_{O} O^{2}+B_{\mu_{L}}^{i} H_{u} L_{i}\right.} \\
& \left.+\frac{1}{3} A_{S} S^{3}+A_{T}^{\lambda} S T^{2}+A_{O}^{\lambda} S O^{2}+A_{S}^{i} S H_{u} L_{i}+A_{T}^{i} H_{u} T L_{i}+\text { h.c }\right]
\end{aligned}
$$

where the terms in the last line are expected to be smaller than those of the first line [128] and will be ignored from now on.

One thing to keep in mind is that the $S U(2)_{L}$ structure of Eq. (6.10) prevents lepton $a$ from normally acquiring a mass. The latter can however come from nonrenormalizable operators that still respect the $U(1)_{R}$ symmetry. For example, this could be [128]

$$
\int d^{4} \theta \frac{X^{\dagger}}{M} H_{u}^{\dagger} L_{a} E_{a} .
$$

\subsection{2 $U(1)_{R_{2}}$ symmetry}

Models of $R=R_{2}$ are the subject of Chapter 8. The superpotential is simply those of Eqs. (6.5), (6.6) and the $R_{2}$ part of Eq. (6.7). The soft terms are given by Eq. (6.8). Most important to us will be the presence of the $U D D$ term in the superpotential. This, combined with the fact that $U(1)_{R}$ is expected to be broken by anomaly mediation or Planck scale suppressed operators, will be the central ingredients to obtaining successful baryogenesis. 


\section{Chapter 7}

\section{Electroweak precision}

\section{measurements in supersymmetric}

\section{models with a $U(1)_{R}$ lepton number}

Chapter 6 stated that Dirac gauginos present many advantages over Majorana ones from a theoretical point of view. One such advantage is that models in which the $U(1)_{R}$ number is associated with the lepton number allow for the reduction of the Higgs sector, which would otherwise need four doublets [116]. This is done by granting the sneutrino a vev, which allows it to play the role of the down-type Higgs. In this setup, the constraints on the vev of the sneutrino are much milder than in traditional supersymmetric models. Indeed, the sneutrino does not carry lepton number and giving it a vev does not introduce unacceptably large neutrino masses. The downtype quarks and leptons then acquire a mass through $R$-parity violating couplings (where here $R$-parity means the conventional $R$-parity, not the $\mathbb{Z}_{2}$ subgroup of the full $U(1)_{R}$ we are imposing on the model). It is well known that such couplings lead to deviations in electroweak precision observables (see Ref. [24] for an exhautive review) 
and can therefore considerably constrain such a model.

Taking these considerations into account, the goal of this chapter is to use electroweak precision observable to constrain a supersymmetric model where the $U(1)_{R}$ symmetry is associated with the lepton number. A preliminary study of such constraints was performed in Refs. [128,130], and LHC phenomenology was studied in Ref. [131]. In Refs. [128, 130], the constraints were estimated by looking at the treelevel effect of the new couplings which were considered in isolation. In this chapter, these constraints are analyzed more thoroughly by considering loop effects as well and fitting to all relevant electroweak data. The operator method of Ref. [5] is adopted to analyze the constraints on the parameter space of the model. Because the model leads to deviations from flavour universality in the lepton sector, it is however necessary to generalize the analysis of Refs. $[5,132]$ to take these effects into account.

This chapter is organized as follows. First, the set of necessary effective operators is presented. Then, their coefficients are expressed in terms of parameters of the model. Corrections to electroweak observables are then calculated in terms of these coefficients. The resulting constraints are then presented.

\subsection{Necessary effective operators for EWPT}

The contribution of new physics to electroweak precision tests (EWPT) is encoded in terms of effective operators. Since all new particles are experimentally constrained to be rather heavy, their contributions can be parametrized to a good approximation in terms of dimension six operators respecting the Standard Model gauge symmetries. We use the same basis as in Ref. [5] but do not assume a full $U(3)^{5}$ flavour symmetry. This symmetry is not present in our model since only one flavour of sneutrino gets a vev. The $U(3)^{3}$ associated with quarks is however preserved. In addition, we do 
not consider operators that lead to flavour changing neutral currents (FCNC) in the lepton or quark sector as those would be much more strongly constrained and can be avoided in our model by appropriate assumptions on the flavour sector.

The operators relevant to EWPT fall in four different categories. The first one is those containing only gauge bosons and Higgs particles [5],

$$
O_{W B}=\left(H^{\dagger} \sigma^{a} H\right) W_{\mu \nu}^{a} B^{\mu \nu}, \quad O_{h}=\left|H^{\dagger} D_{\mu} H\right|^{2} .
$$

As far as EWPT are concerned, the Higgs field appearing in these operators is only relevant for its vev. These operators are therefore related to modifications of the gauge bosons' two-point functions.

The second category of operators are four-fermion operators. Those that are relevant to EWPT are [5]

$$
\begin{aligned}
O_{L L}^{s}[m n] & =\frac{1}{2}\left(\bar{L}^{m} \gamma^{\mu} L^{m}\right)\left(\bar{L}^{n} \gamma_{\mu} L^{n}\right), & O_{L L}^{t}[m n] & =\frac{1}{2}\left(\bar{L}^{m} \sigma^{a} \gamma^{\mu} L^{m}\right)\left(\bar{L}^{n} \sigma^{a} \gamma_{\mu} L^{n}\right), \\
O_{L Q}^{s}[m] & =\left(\bar{L}^{m} \gamma^{\mu} L^{m}\right)\left(\bar{Q} \gamma_{\mu} Q\right), & O_{L Q}^{t}[m] & =\left(\bar{L}^{m} \sigma^{a} \gamma^{\mu} L^{m}\right)\left(\bar{Q} \sigma^{a} \gamma_{\mu} Q\right), \\
O_{L E}[m n] & =\left(\bar{L}^{m} \gamma^{\mu} L^{m}\right)\left(\bar{E}^{n} \gamma_{\mu} E^{n}\right), & & O_{Q E}[m]=\left(\bar{Q} \gamma^{\mu} Q\right)\left(\bar{E}^{m} \gamma_{\mu} E^{m}\right), \\
O_{L U}[m] & =\left(\bar{L}^{m} \gamma^{\mu} L^{m}\right)\left(\bar{U} \gamma_{\mu} U\right), & O_{L D}[m] & =\left(\bar{L}^{m} \gamma^{\mu} L^{m}\right)\left(\bar{D} \gamma_{\mu} D\right), \\
O_{E E}[m n] & =\frac{1}{2}\left(\bar{E}^{m} \gamma^{\mu} E^{m}\right)\left(\bar{E}^{n} \gamma_{\mu} E^{n}\right), & & O_{E U}[m]=\left(\bar{E}^{m} \gamma^{\mu} E^{m}\right)\left(\bar{U} \gamma_{\mu} U\right), \\
O_{E D}[m] & =\left(\bar{E}^{m} \gamma^{\mu} E^{m}\right)\left(\bar{D} \gamma_{\mu} D\right) . & &
\end{aligned}
$$

Lepton flavour indices are shown explicitly, while quark flavour indices are summed implicitly as they respect the unbroken $U(3)^{3}$. Four-fermion operators containing four quarks do not affect any of the observables considered in this work and are therefore ignored.

The third category of operators are those containing both the Higgs field and 
fermions $[5],{ }^{1}$

$$
\begin{aligned}
O_{H L}^{s}[m] & =i\left(H^{\dagger} D^{\mu} H\right)\left(\bar{L}^{m} \gamma_{\mu} L^{m}\right)+\text { h.c., } & O_{H L}^{t}[m] & =i\left(H^{\dagger} \sigma^{a} D^{\mu} H\right)\left(\bar{L}^{m} \sigma^{a} \gamma_{\mu} L^{m}\right)+\text { h.c., } \\
O_{H Q}^{s} & =i\left(H^{\dagger} D^{\mu} H\right)\left(\bar{Q} \gamma_{\mu} Q\right)+\text { h.c., } & O_{H Q}^{t} & =i\left(H^{\dagger} \sigma^{a} D^{\mu} H\right)\left(\bar{Q} \sigma^{a} \gamma_{\mu} Q\right)+\text { h.c., } \\
O_{H E}[m] & =i\left(H^{\dagger} D^{\mu} H\right)\left(\bar{E}^{m} \gamma_{\mu} E^{m}\right)+\text { h.c., } & & O_{H U}=i\left(H^{\dagger} D^{\mu} H\right)\left(\bar{U} \gamma_{\mu} U\right)+\text { h.c. } \\
O_{H D} & =i\left(H^{\dagger} D^{\mu} H\right)\left(\bar{D} \gamma_{\mu} D\right)+\text { h.c. } & &
\end{aligned}
$$

Once again, the Higgs field is only relevant for its vev. These operators affect the vertices containing a gauge boson and two fermions.

Finally, there is one operator affecting only gauge boson self-interactions [5],

$$
O_{\hat{W}}=\epsilon^{a b c} W_{\mu}^{a \nu} W_{\nu}^{b \lambda} W_{\lambda}^{c \mu}
$$

The total effective Lagrangian is therefore the sum of the Lagrangian of the SM and a linear combination of the different dimension six operators,

$$
\mathcal{L}=\mathcal{L}_{\mathrm{SM}}+a_{i} O_{i}
$$

where $O_{i}$ represent the operators, and $a_{i}$ are coefficients with dimension of inverse mass squared.

\subsection{Coefficients of the EWPT operators}

In this section, the contributions to the different operators are presented. We first compute the coefficients of operators related to oblique corrections. Then, the four-

\footnotetext{
${ }^{1}$ Because this is a two Higgs doublet model, one could also write operators with the second doublet. However, the effects of these on precision observables can be absorbed in the operators of Eq. (7.3).
} 
fermion operators coming from scalar exchange and box diagrams are presented. Operators that modify gauge boson vertices coming from loop diagrams and mixing are shown. Finally, the contributions to the operator $O_{\hat{W}}$ are discussed.

\subsubsection{Oblique parameters}

A standard and convenient way of parametrizing deviations to EWPT is through the so-called oblique parameters [133,134], which are defined as modifications to twopoint functions of the electroweak gauge bosons. The coefficients of some of the higher dimensional operators mentioned above can in turn be written as a function of these parameters. In our model, the oblique parameters are generated by the vev of the third component of the triplet and by loop diagrams.

The definitions of Ref. [6] are adopted for the oblique parameters $\hat{S}, \hat{T}, Y$ and $W$ (see also Ref. [135]),

$$
\begin{aligned}
\hat{S} & =\frac{g}{g^{\prime}} \Pi_{W_{3} B}^{\prime}(0), & \hat{T} & =\frac{\Pi_{W_{3} W_{3}}(0)-\Pi_{W^{+} W^{-}}(0)}{M_{W}^{2}}, \\
Y & =\frac{M_{W}^{2}}{2} \Pi_{B B}^{\prime \prime}(0), & W & =\frac{M_{W}^{2}}{2} \Pi_{W_{3} W_{3}}^{\prime \prime}(0),
\end{aligned}
$$

where the $\Pi\left(q^{2}\right)$ 's are the two-point functions, $q$ is the momemtum of the incoming particle and the primes represent derivatives with respect to $q^{2}$. Following Ref. [6], loop-level contributions to these parameters are calculated by considering diagrams of the form shown in Fig. 7.1, where the vevs are treated as perturbations.

The parameters $\hat{S}$ and $\hat{T}$ can be related to $O_{W B}$ and $O_{H}$ by a simple matching procedure,

$$
a_{W B}=\frac{g^{\prime} \hat{S}}{g v^{2}}, \quad a_{H}=-\frac{2 \hat{T}}{v^{2}} .
$$

The two other oblique coefficients are related to operators which are not listed in Eq. 


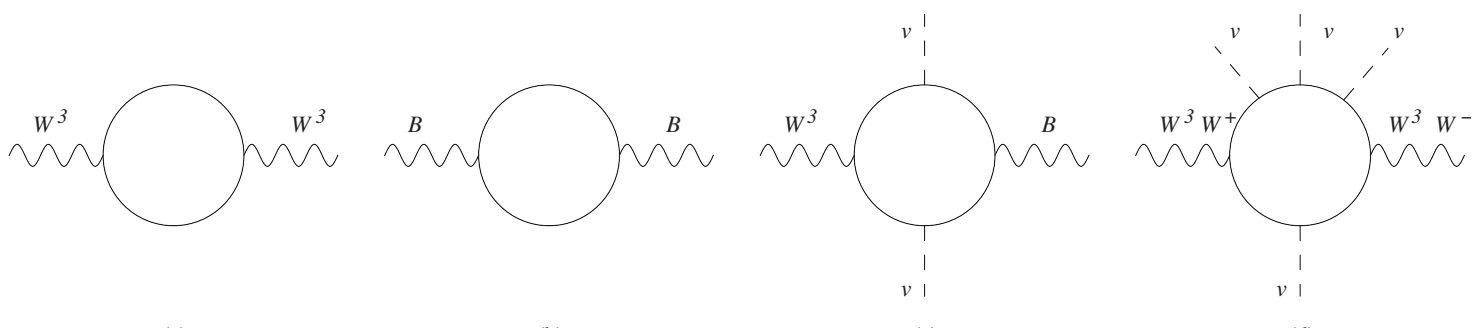

(a)

(b)

(c)

(d)

Figure 7.1: Diagrams contributing to the oblique parameters [6]. Plain lines correspond to unspecified superpartners or leptons $a$. (a), (b), (c) and (d) correspond to $W, Y, \hat{S}$ and $\hat{T}$ respectively. $v$ stands for the insertion of a vev.

$$
O_{Y}=\frac{\left(\partial_{\rho} B_{\mu \nu}\right)^{2}}{2}, \quad O_{W}=\frac{\left(D_{\rho} W_{\mu \nu}\right)^{2}}{2}
$$

Using the equations of motions, these operators can be written in terms of the operators shown in Eqs. (7.1), (7.2) and (7.3) (plus additional operators that do not contribute to EWPT). The only operator generated by the elimination of $O_{Y}$ that falls in the category of Eq. (7.1) is

$$
\left(H^{\dagger} D^{\mu} H+\text { h.c. }\right)^{2} \text {. }
$$

This operator can be related to $O_{H}$ and terms irrelevant to EWPT. The net effect on $a_{H}$ due to eliminating $O_{Y}$ is

$$
\left.a_{H}\right|_{Y}=-\frac{g^{\prime 2} Y}{4 M_{W}^{2}} .
$$

In practice, this contribution is overshadowed by the $\hat{T}$ term. The operator $O_{W}$ can also be eliminated using the equations of motion, but does not give any contributions to the operators of Eq. (7.1). 


\section{Vev of $T^{3}$}

In general, the field $T^{3}$ will acquire a vev of the form

$$
v_{T^{3}}=\frac{g M_{2}^{D}\left(v_{u}^{2}-v_{a}^{2}\right)-\sqrt{2} \lambda_{T} \mu v_{u}^{2}}{2\left(m_{T_{R}}^{2}+\frac{\left(\lambda_{T}\right)^{2} v_{u}^{2}}{2}\right)},
$$

where $m_{T_{R}}^{2} \equiv m_{T}^{2}+b_{T}+4\left(M_{2}^{D}\right)^{2}$ and $v_{u}$ and $v_{a}$ are the vevs of the neutral components of $h_{u}$ and $l_{a}$ respectively. This will give a tree-level contribution to $\hat{T}$ through a correction to the charged $W$ boson mass,

$$
\hat{T}_{v_{T^{3}}}=\frac{4 v_{T^{3}}^{2}}{v^{2}}
$$

For a light enough scalar triplet, this contribution dominates the $\hat{T}$ parameter. The singlet also acquires a small vev. However, it is not a direct contribution and its effect is therefore much smaller and can be ignored.

\section{Scalars}

Loops of scalars will contribute to the oblique parameters. The relevant couplings come from Eqs. (6.4), (6.5) and (6.6). The first term of each line of Eq. (6.4) is present in the MSSM, and these lead to contributions similar to those presented in Ref. [6].

For sfermions, the results of Ref. [6] apply almost directly. The only difference is that, as $l_{a}$ provides mass to the down type quarks and leptons, we include its scalar contributions in the Higgs sector. This has the advantage of making expressions 
simpler and easier to compare. The sfermions contributions are

$$
\begin{aligned}
W_{\text {sfermions }} & =\frac{\alpha_{2} M_{W}^{2}}{80 \pi}\left(\frac{2}{3} \frac{1}{m_{L}^{2}}+\frac{3}{m_{Q}^{2}}\right), \\
Y_{\text {sfermions }} & =\frac{\alpha_{Y} M_{W}^{2}}{40 \pi}\left(\frac{4}{3} \frac{Y_{L}^{2}}{m_{L}^{2}}+\frac{Y_{E}^{2}}{m_{E}^{2}}+3 \frac{Y_{D}^{2}}{m_{D}^{2}}+3 \frac{Y_{U}^{2}}{m_{U}^{2}}+6 \frac{Y_{Q}^{2}}{m_{Q}^{2}}\right), \\
\hat{S}_{\text {sfermions }} & =-\frac{\alpha_{2}}{24 \pi}\left[M_{W}^{2}\left(2 \frac{Y_{L}}{m_{L}^{2}}+9 \frac{Y_{Q}}{m_{Q}^{2}}\right)+\frac{1}{2} \frac{m_{t}^{2}}{m_{Q}^{2}}\right], \\
\hat{T}_{\text {sfermions }} & =\frac{\alpha_{2} M_{W}^{2}}{16 \pi} \cos ^{2} 2 \beta\left(\frac{2}{3} \frac{1}{m_{L}^{2}}+\frac{2}{m_{Q}^{2}}\right)+\hat{T}_{\text {stop }},
\end{aligned}
$$

where $\tan \beta=v_{u} / v_{a}$ and $\hat{T}_{\text {stop }}$ is given by [6]

$$
\hat{T}_{\text {stop }}=\frac{\alpha_{2}}{16 \pi} \frac{\left(M_{W}^{2} \cos ^{2} 2 \beta+m_{t}^{2}\right)^{2}}{m_{Q}^{2} M_{W}^{2}} .
$$

The $R_{d}$ scalar field, despite not acquiring a vev, still contributes to the oblique parameters. For $Y$ and $W$, its contributions are similar to those of $l_{b}$ and $l_{c}$ and can be read from Eq. (7.13) directly. For $\hat{S}$ and $\hat{T}$, the presence of $W_{\text {adj }}$ changes the result. The parameters are

$$
\begin{aligned}
W_{R_{d}} & =\frac{\alpha_{2} M_{W}^{2}}{240 \pi M_{R_{d}}^{2}}, \\
Y_{R_{d}} & =\frac{\alpha_{Y} M_{W}^{2}}{240 \pi M_{R_{d}}^{2}}, \\
\hat{S}_{R_{d}} & =\frac{\alpha_{2} M_{W}^{2}}{48 \pi M_{R_{d}}^{2}}\left(\cos 2 \beta-\frac{2 \sin ^{2} \beta\left(\lambda_{T}^{2}-\lambda_{S}^{2}\right)}{g^{2}}\right), \\
\hat{T}_{R_{d}} & =\frac{\alpha_{2} M_{W}^{2}}{48 \pi M_{R_{d}}^{2}}\left(\cos 2 \beta-\frac{2 \sin ^{2} \beta\left(\lambda_{T}^{2}-\lambda_{S}^{2}\right)}{g^{2}}\right)^{2},
\end{aligned}
$$

where $M_{R_{d}}^{2} \equiv \mu^{2}+m_{R_{d}}^{2}$. The Higgs sector (including the scalar part of $l_{a}$ ) gives contributions to $\hat{S}, W$ and $Y$ that can once again be obtained by using the results of 
Ref. [6] almost directly. In terms of $m_{A^{0}}^{2}=m_{H_{u}}^{2}+m_{L}^{2}+\mu^{2}$, these contributions are

$$
\begin{aligned}
W_{\text {Higgs }} & =\frac{\alpha_{2}}{240 \pi} \frac{M_{W}^{2}}{m_{A^{0}}^{2}}, \\
Y_{\text {Higgs }} & =\frac{\alpha_{Y}}{240 \pi} \frac{M_{W}^{2}}{m_{A^{0}}^{2}}, \\
\hat{S}_{\text {Higgs }} & =-\frac{\alpha_{2}}{48 \pi} \frac{M_{W}^{2}}{m_{A^{0}}^{2}}\left(1-\frac{M_{Z}^{2}}{2 M_{W}^{2}} \sin ^{2} 2 \beta\right) .
\end{aligned}
$$

The presence of the scalar components of the singlet and triplet do not lead to any contribution to $Y$ and $\hat{S}$ by themselves, but only a contribution to $W$,

$$
W_{\text {scalar gauge }}=\frac{\alpha_{2} M_{W}^{2}}{120 \pi}\left(\frac{1}{m_{T_{R}}^{2}}+\frac{1}{m_{T_{I}}^{2}}\right),
$$

where $m_{T_{I}}^{2} \equiv m_{T}^{2}-b_{T}$. The contribution to $\hat{T}$ from diagrams with Higgs, triplet and singlets is however more difficult to compute using insertions of the Higgs vev because of the mixing between the Higgs and the triplet. Therefore, we compute these contributions by numerically diagonalizing the scalar mass matrix.

\section{Higgsinos and gauginos}

The fit constrains the value of $v_{a}$ to a region of phase space where it is much smaller than $v_{u}$. As such, the contributions to the oblique parameters containing only powers of $v_{u}$ dominate and are presented here. The contributions containing powers of $v_{a}$ were also included in the numerical fit. With the exception of the contributions coming from couplings $\lambda_{S}$ and $\lambda_{T}$, the diagrams with binos are usually smaller by an order of magnitude or so and are not presented for simplicity, but were included in the fit. 
The dominant terms in the limit of $\lambda_{S}$ and $\lambda_{T}$ small are

$$
\begin{aligned}
W_{\text {fermions }} & =\frac{\alpha_{2}}{30 \pi}\left(\frac{4 M_{W}^{2}}{\left(M_{2}^{D}\right)^{2}}+\frac{M_{W}^{2}}{\mu^{2}}\right) \\
Y_{\text {fermions }} & =\frac{\alpha_{Y} M_{W}^{2}}{30 \pi \mu^{2}} \\
S_{\text {fermions }} & =\frac{\alpha_{2} M_{W}^{2}}{12 \pi\left(M_{2}^{D}\right)^{2}}\left[\frac{a\left(a-5-2 a^{2}\right)}{(a-1)^{4}}+\frac{\left(1-2 a+9 a^{2}-4 a^{3}+2 a^{4}\right)}{(a-1)^{5}} \ln a\right] \sin ^{2} \beta, \\
T_{\text {fermions }} & =\frac{\alpha_{2} M_{W}^{2}}{48 \pi\left(M_{2}^{D}\right)^{2}}\left[\frac{19-64 a-91 a^{2}+16 a^{3}}{(a-1)^{4}}+\frac{6 a\left(-4+25 a-a^{2}\right)}{(a-1)^{5}} \ln a\right] \sin ^{4} \beta,
\end{aligned}
$$

where $a \equiv\left(\mu / M_{2}^{D}\right)^{2}$. The oblique parameters containing $\lambda_{S}$ and $\lambda_{T}$ are included but not presented here as the expressions are rather long. The contribution to the parameter $\hat{T}$ is in fact the dominant term in setting limits on $\lambda_{S}$ and $\lambda_{T}$ for massive enough scalar gauge particles.

\subsubsection{Four-fermion operators}

There are many contributions to the four-fermion operators. These can come from the operators in Eq. (7.8) associated with $W$ and $Y$ once the equations of motion are used, from scalar exchange and from box diagrams. The contributions proportional to $W$ and $Y$ are given by

$$
a_{i j}^{s}=-Y_{i} Y_{j} g^{\prime 2} \frac{Y}{2 M_{W}^{2}}, \quad a_{i j}^{t}=-g^{2} \frac{W}{8 M_{W}^{2}},
$$

where $i$ and $j$ stand for the different combinations of fields possible. The family indices are suppressed because they are the same for every combination, as is expected from the fact that the oblique parameters are universal.

The tree-level scalar exchange contributions come from the exchange of sfermions between leptons or down quarks. The scalars are integrated out and the diagrams 


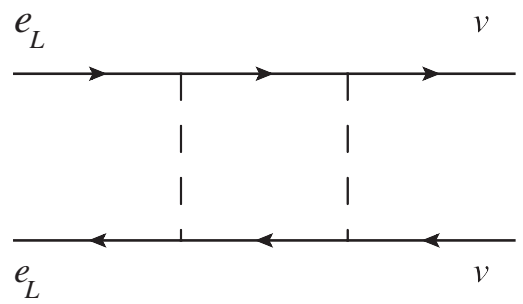

Figure 7.2: Diagram contributing to the four-fermion operators. Unidentified lines correspond to unspecified superpartners.

are rewritten using the Fiertz rearrangement formulas. In practice, most of them have a negligible effect on the fit as they involve small Yukawa couplings. The only important ones are those which contribute to the observables $R_{\tau}$ and $R_{\tau \mu}$ [136]. They are

$$
a_{L L}^{s}[a b]=\frac{y_{b}^{2}}{4 m_{E}^{2}}, \quad a_{L L}^{s}[a c]=\frac{y_{c}^{2}}{4 m_{E}^{2}},
$$

where $y_{b}$ and $y_{c}$ are the Yukawa coupling of leptons $b$ and $c$ respectively. Note that being proportional to lepton Yukawa couplings, the contributions to these operators increases as $v_{a}$ is lowered and will lead to a lower (upper) bound on $v_{a}(\tan \beta)$.

Box diagrams like those of Fig. 7.2 are also accounted for with four-fermion operators, though the expressions are too long to be included here. The fact that all combinations of particles entering the box diagrams can be taken into account with the far fewer operators of Eq. (7.8) is not trivial and requires several relations to be satisfied. This serves as a consistency check. In the case of diagrams including an exchange of sleptons $a$, the limit of large $\tan \beta$ is also taken, as it is strongly overshadowed by mixing effects anyway. 


\subsubsection{Vertex modifying operators}

The operators of Eq. (7.3) receive contributions from lepton mixing, oblique parameters $(W$ and $Y$ ) and loop diagrams. Because the left-handed parts of the leptons of family $a$ mix with superpartners of different gauge charges, the interactions between the gauge bosons and the physical leptons are modified. The result is readily obtained by replacing the leptons of family $a$ and the relevant superpartners by their equivalent combinations of mass eigenstates in the gauge boson vertex interaction terms. This can be summarized in terms of effective operators with coefficients

$$
a_{H L}^{t}[a]=\frac{1}{8 v^{2}}\left[\left(\frac{g v_{a}}{M_{2}^{D}}\right)^{2}-\left(\frac{g^{\prime} v_{a}}{M_{1}^{D}}\right)^{2}\right], \quad a_{H L}^{s}[a]=\frac{1}{8 v^{2}}\left[3\left(\frac{g v_{a}}{M_{2}^{D}}\right)^{2}+\left(\frac{g^{\prime} v_{a}}{M_{1}^{D}}\right)^{2}\right] .
$$

These relations are non-trivial as they have to take into account interactions of leptons of family $a$ with photons, $W$ 's and $Z$ 's with the help of only two free parameters. The right-handed part of the lepton of family $a$ mixes as well, but this effects is suppressed with respect to left-handed mixing by a factor of $\left(m_{a} / M_{2}^{D}\right)^{2}$ and is therefore ignored. These operators lead to deviations of the coupling of the lepton of flavour $a$ to the SM gauge bosons, and will lead to an upper (lower) bound on $v_{a}(\tan \beta)$.

The contributions from oblique parameters that arise once $O_{Y}$ and $O_{W}$ are eliminated are given by

$$
a_{H i}^{s}=-Y_{i} g^{2} \frac{Y}{4 M_{W}^{2}}, \quad a_{H j}^{t}=-g^{2} \frac{W}{8 M_{W}^{2}} .
$$

Once again, the family indices are suppressed because they are the same for every generation.

Finally, loop-level diagrams of the type shown in Fig. 7.3 also lead to corrections 
to the gauge boson fermion vertices. They appear in the four possible ways shown. We can parametrize their effects in terms of ${ }^{2}$

$$
\begin{aligned}
& A_{1}=-2 \sum \text { diagrams of type (a), } \\
& A_{2}=2 \sum \text { diagrams of type (b), } \\
& A_{3}=-\sqrt{2} \sum \text { diagrams of type (c), } \\
& A_{4}=2 \sum \text { diagrams of type (d). }
\end{aligned}
$$

It can be shown that these quantities are related by $A_{1}+A_{2}=2 A_{3}$. This relation can also be shown to be a consequence of the fact that only three operators in Fig. 7.3 correspond to four types of diagrams. The fact that it is respected is highly nontrivial. Using this relation, the diagrams can be accounted for by using the operators of Eq. (7.3). This leads to coefficients of

$$
a_{H L}^{s}=\frac{A_{2}-A_{3}}{g v^{2}}, \quad a_{H L}^{t}=\frac{A_{3}}{g v^{2}}, \quad a_{H E}=\frac{A_{4}}{g v^{2}} .
$$

The same equations also apply to quarks. The diagrams containing Yukawa coupling are neglected as these are considerably smaller. The limit of large $\tan \beta$ is also taken when an internal slepton $a$ is present.

\subsubsection{Loop contributions to $O_{\hat{W}}$}

The contributions to the operator $O_{\hat{W}}$ arise at loop-level. As this operator leads only to triple gauge boson interactions, it is very poorly constrained and its effect on the fit is negligible. It is therefore not included.

\footnotetext{
${ }^{2}$ To avoid a possible sign confusion, we mention that covariant derivatives are taken with a + sign. For example, $D_{\mu} E=\left(\partial_{\mu}+i g^{\prime} Y_{E} B_{\mu}\right) E$.
} 


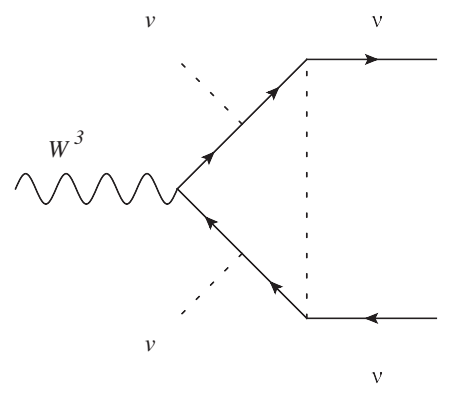

(a)

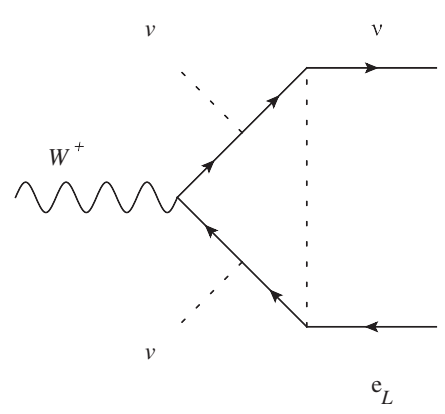

(c)

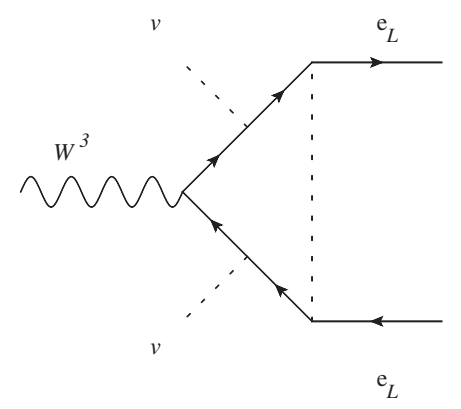

(b)

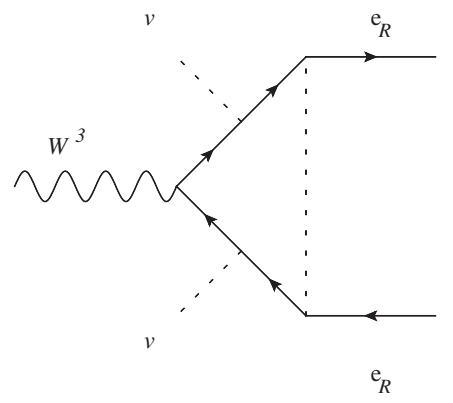

(d)

Figure 7.3: Corrections to gauge interactions arising from loops. Unidentified lines correspond to unspecified superpartners or leptons $a$. There are four possible types and similar diagrams exist for quarks. 


\subsection{Method and results}

\begin{tabular}{|c|c|c|c|}
\hline & Observable & Standard Notation & Reference \\
\hline $\begin{array}{l}\text { Atomic parity } \\
\text { violation }\end{array}$ & $\begin{array}{l}\text { Weak charge in Cs } \\
\text { Weak charge in } \mathrm{Tl}\end{array}$ & $\begin{array}{l}Q_{W}(C s) \\
Q_{W}(T l)\end{array}$ & $\begin{array}{c}{[137]} \\
{[138,139]}\end{array}$ \\
\hline DIS & $\begin{array}{c}\nu_{\mu} \text {-nucleon scattering from NuTeV } \\
\nu \text {-e scattering from CHARM II } \\
\nu_{\mu} \text {-nucleon scattering from CDHS and CHARM } \\
\nu_{\mu} \text {-nucleon scattering from CCFR }\end{array}$ & $\begin{array}{l}g_{L}^{2}, g_{R}^{2} \\
g_{V}^{\nu e}, g_{A}^{\nu e} \\
\quad R^{\nu} \\
\quad \kappa\end{array}$ & $\begin{array}{c}{[140]} \\
{[141]} \\
{[142,143]} \\
{[144]}\end{array}$ \\
\hline $\begin{array}{l}\text { Fermion pair } \\
\text { production at } \\
\text { LEP2 }\end{array}$ & $\begin{array}{c}\text { Forward-backward asymmetries for } e^{+} e^{-} \rightarrow f \bar{f} \\
\text { Total cross section for } e^{+} e^{-} \rightarrow f \bar{f} \\
\text { Differential cross section for } e^{+} e^{-} \rightarrow e^{+} e^{-}\end{array}$ & $\begin{array}{c}A_{F B}^{f}(f=e, \mu, \tau, b, c) \\
\sigma_{f}(f=q, \mu, \tau) \\
d \sigma_{e} / d \cos \theta\end{array}$ & $\begin{array}{l}{[145]} \\
{[145]} \\
{[146]}\end{array}$ \\
\hline & $W$ mass & $M_{W}$ & {$[145,147]$} \\
\hline $\begin{array}{c}\text { Ratio of lepton } \\
\text { decay rate }\end{array}$ & $\begin{array}{l}\text { Ratio of decay rate of } \tau \text { to } e \text { on } \tau \text { to } \mu \\
\text { Ratio of decay rate of } \tau \text { to } \mu \text { on } \mu \text { to } e\end{array}$ & $\begin{array}{l}R_{\tau} \\
R_{\tau \mu}\end{array}$ & [136] \\
\hline$W$ pair & Differential cross section for $e^{+} e^{-} \rightarrow W^{+} W^{-}$ & $d \sigma_{W} / d \cos \theta$ & [148] \\
\hline Z-pole & $\begin{array}{c}\text { Polarized asymmetries } \\
\text { Forward-backward asymmetries } \\
\text { Ratios of decay rates } \\
\text { Total Z width } \\
e^{+} e^{-} \text {hadronic cross section at Z pole }\end{array}$ & $\begin{array}{c}A_{f}(f=e, \mu, \tau, b, c) \\
A_{F B}^{0, f}(f=e, \mu, \tau, b, c) \\
R_{f}^{0}(f=e, \mu, \tau, b, c) \\
\Gamma_{Z} \\
\sigma_{h}^{0}\end{array}$ & $\begin{array}{l}{[145]} \\
{[145]} \\
{[145]} \\
{[145]} \\
{[145]}\end{array}$ \\
\hline
\end{tabular}

Table 7.1: Relevant observables. They are a modified list of those of Ref. [5].

The coefficients of the different operators are constrained using the observables of Table 7.1. They are those of Ref. [5] with a few minor differences. The observable $\sin \theta_{\text {eff }}$ is not used because it assumes lepton universality which is not the case with the model. The ratios of decay rates $R_{\tau}$ and $R_{\tau \mu}$ [136] are included, as they affect strongly the lower limit on $v_{a}$. These two observables would be unaffected if $U(3)^{5}$ symmetry was assumed.

The correction to each observable is calculated to linear order in the coefficients of the higher dimensional operators and this is used to calculate the $\chi^{2}$ distribution. Each coefficient is replaced by its expression in terms of the parameters of the theory. It is then possible to set a number of parameters and do a fit on the remaining ones. These parameters are then constrained inside a region of phase space with a given confidence level. For the set of masses considered (shown in Table 7.2), it is found 


\begin{tabular}{|c|c|c|c|c|c|c|c|c|}
\hline Figure & $a$ & $M_{\text {sfermions }}$ & $m_{R_{d}}$ & $\mu$ & $m_{T}, m_{S}$ & $M_{2}^{D}, M_{1}^{D}$ & $\lambda_{S}$ & $\chi^{2} /$ D.O.F. \\
\hline $7.4 \mathrm{a}$ & $e$ & 500 & 700 & $\begin{array}{l}200 \\
400 \\
600\end{array}$ & 1000 & 700 & Variable & $\begin{array}{l}0.916 \\
0.883 \\
0.878\end{array}$ \\
\hline $7.4 \mathrm{~b}$ & $e$ & 500 & 700 & $\begin{array}{l}200 \\
400 \\
600\end{array}$ & 1000 & 1000 & Variable & $\begin{array}{l}0.909 \\
0.883 \\
0.879\end{array}$ \\
\hline $7.5 \mathrm{a}$ & $\begin{array}{l}e \\
\mu \\
\tau\end{array}$ & 500 & 700 & 400 & 1000 & 700 & 0 & $\begin{array}{l}0.879 \\
0.881 \\
0.880\end{array}$ \\
\hline $7.5 \mathrm{~b}$ & $\begin{array}{l}e \\
\mu \\
\tau\end{array}$ & 500 & 700 & 400 & 1000 & 1000 & 0 & $\begin{array}{l}0.881 \\
0.882 \\
0.881\end{array}$ \\
\hline 7.6a, 7.6b & $\mathrm{N} / \mathrm{A}$ & 500 & 700 & 400 & 1000 & $\begin{array}{c}500 \\
700 \\
1000\end{array}$ & 0 & $\mathrm{~N} / \mathrm{A}$ \\
\hline
\end{tabular}

Table 7.2: Masses for each plot in $\mathrm{GeV}$ and choice of lepton $a . \chi^{2} /$ D.O.F. stands for the best fit of $\chi^{2}$ divided by the number of degrees of freedom. For the Standard Model, $\chi^{2} /$ D.O.F is 0.883. In all cases, $b_{T}=b_{S}=0$.

that fits of our model to the data are roughly as good as the Standard Model fit. As mentioned above, the vev for the triplet $T^{3}$ is potentially dangerous so the mass parameter of the scalar triplet is restricted to be above $1 \mathrm{TeV}$. The result of the $\chi^{2}$ per degree of freedom for different choices of parameters is also shown in Table 7.2. The allowed region of parameter space, in the $\lambda_{S} / \lambda_{T} / v_{a}$ space consists of a roughly cylindrical region whose flat sides are parallel to the $\lambda_{T} / \lambda_{S}$ plane. The $\hat{T}$ parameter has contributions that scale as $\lambda_{T}^{4}$ and $\lambda_{S}^{4}$ and is the dominant factor in determining the shape of the allowed region for $\lambda_{T}$ and $\lambda_{S}$. Fig. 7.4 shows this region of allowed phase space in the $\lambda_{S} / \lambda_{T}$ plane for different combinations of masses which can be found in Table 7.2. Only the $95.45 \%$ confidence level exclusion is shown as the lines of confidence level of $68.27 \%$ and $99.76 \%$ are very close to those of $95.45 \%$ and provide little new information. We see that there are strong bounds on those parameters, with the allowed region becoming larger as $M_{(1,2)}^{D}$ is increased as it sets the scale of $v_{T^{3}}$ and 


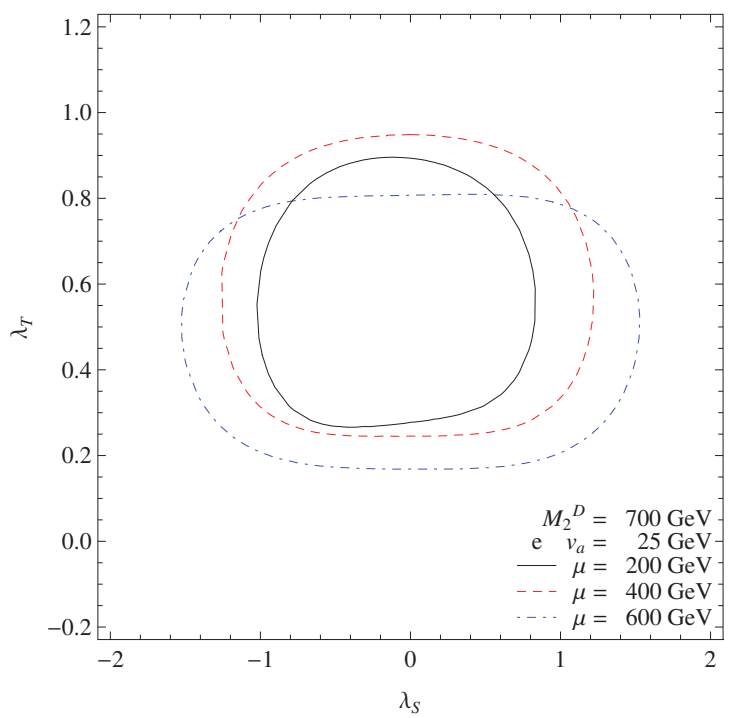

(a)

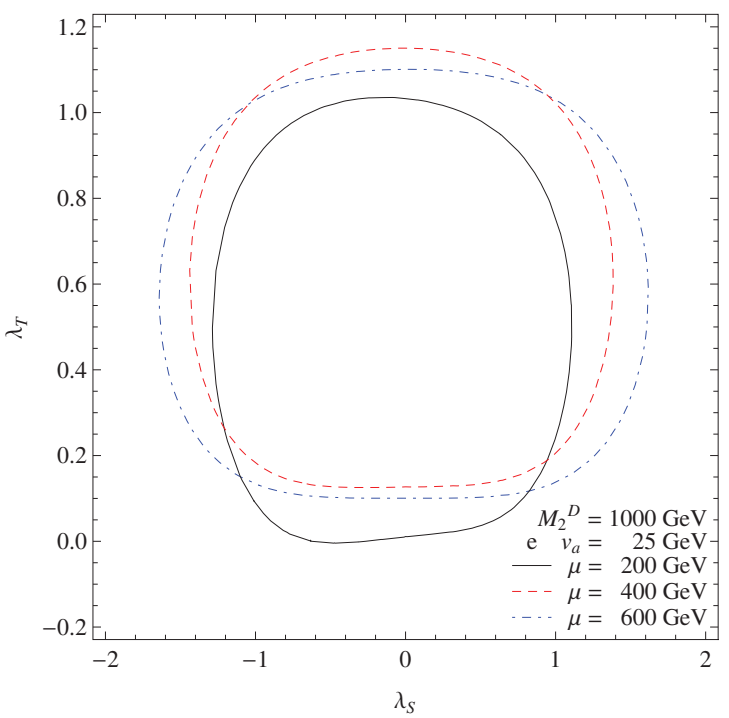

(b)

Figure 7.4: Region of allowed phase space for the mass combinations of Table 7.2 and for different values of $\mu$. The solid, dashed and dotdashed lines correspond respectively to 200,400 and $600 \mathrm{GeV}$. (a) is taken for $M_{1}^{D}=M_{2}^{D}=700 \mathrm{GeV}$ and (b) for $M_{1}^{D}=M_{2}^{D}=1000 \mathrm{GeV}$. Both are taken at $v_{a}=25 \mathrm{GeV}$ and lepton $a$ is the electron. The contours correspond to $95.45 \%$ confidence level.

the masses of the fermions which give the largest loop contributions to $\hat{T}$. Overall, we see that $\lambda_{S}$ and $\lambda_{T}$ cannot take values much greater than one irrespective of the masses of the superpartner or the choice of generation for the lepton $a$. This has important consequences for radiative corrections to the Higgs mass in this model [149]. As explained before, the contributions of Eqs. (7.20) and (7.21) lead to a two-sided bound on $v_{a}$ which is constrained to be rather small, though the exact range depends considerably on the choice of masses and the generation of the lepton $a$. This is illustrated in Fig. 7.5 which shows the allowed region in the $\lambda_{T} / v_{a}$ plane. Again, the upper bound on $v_{a}$ is relaxed as $M_{(1,2)}^{D}$ is increased and can reach a value where $\tan \beta=2$ for $a=\tau$ and $M_{(1,2)}^{D}=1000 \mathrm{GeV}$. The lower bound on $v_{a}$ primarily depends on the mass of the sfermions which are not varied in the figures. In Fig. 7.6, the $\hat{T}$ and $\hat{S}$ parameters are shown as a function of $\lambda_{T}$. We see that the $\hat{S}$ parameter is very 


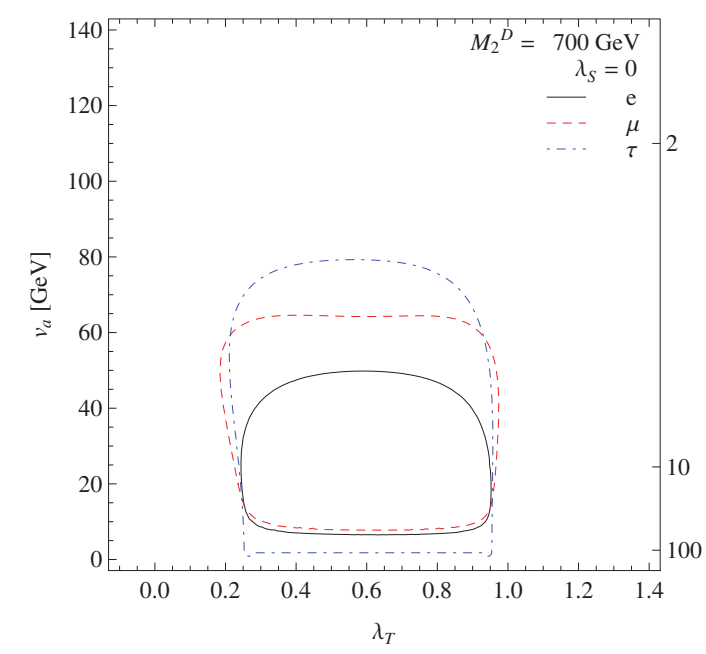

(a)

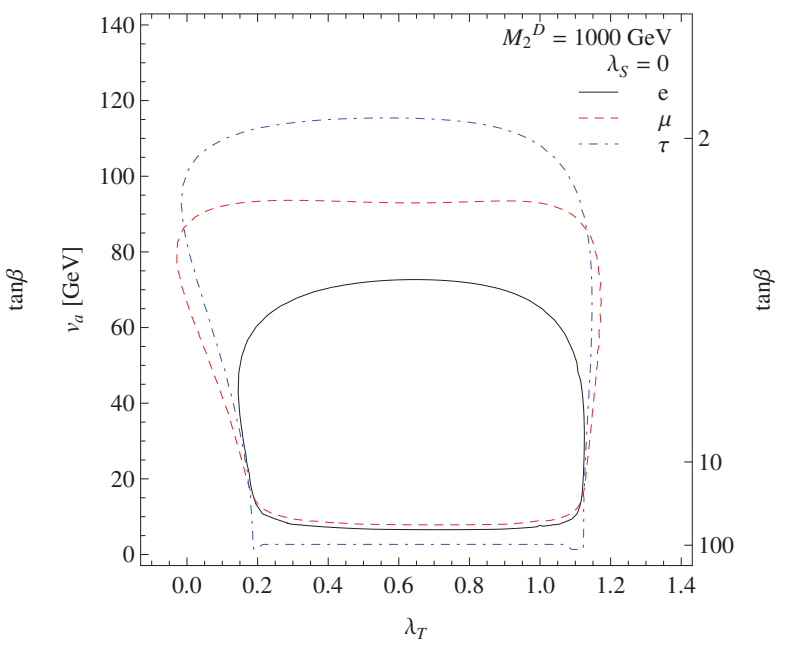

(b)

Figure 7.5: Region of allowed phase space for the mass combinations of Table 7.2 and for different choices of lepton $a$ The solid, dashed and dotdashed lines correspond respectively to the electron, muon and tau. Both figures have $\lambda_{S}=0$ and (a) has $M_{1}^{D}=M_{2}^{D}=700 \mathrm{GeV}$ and (b) has $M_{1}^{D}=M_{2}^{D}=1000 \mathrm{GeV}$. The contours correspond to $95.45 \%$ confidence level.

small while the $\hat{T}$ parameter can become sizable and drives the limit on $\lambda_{T}$.

Overall, as one would expect, increasing the various mass parameters will relax the various bounds. The situation for $\mu$ is however slightly more involved as increasing $\mu$ will increase $v_{T^{3}}$ (see Eq. (7.11)) which can then be taken back to an acceptable value by constraining $\lambda_{T}$ to be close to $\lambda_{T} \sim g M_{2}^{D}\left(v_{u}^{2}-v_{a}^{2}\right) /\left(\sqrt{2} \mu v_{u}^{2}\right)$. 


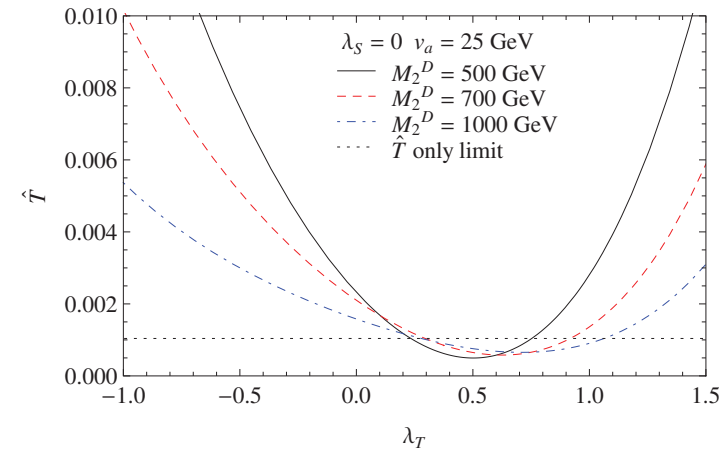

(a)

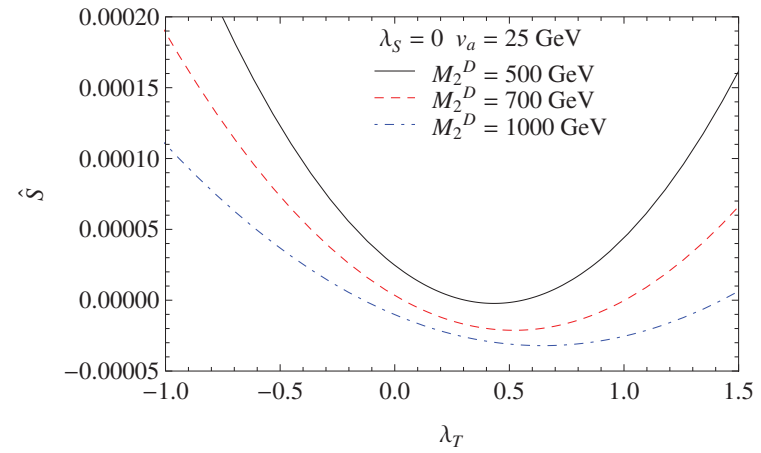

(b)

Figure 7.6: $\hat{T}$ and $\hat{S}$ parameters as a function of $\lambda_{T}$ for the masses of Table 7.2 and different values of $M_{2}^{D}$. The solid, dashed and dotdashed lines correspond respectively to 500,700 and $1000 \mathrm{GeV}$. Both are taken at $v_{a}=25 \mathrm{GeV}, M_{1}^{D}=M_{2}^{D}$ and with $\lambda_{S}=0$. The horizontal line corresponds to what the limit on $\hat{T}$ would be at $95.45 \%$ if only its corresponding operator would be present and $Y=0$. Its value is $1.04 \times$ $10^{-3}$. 


\section{Chapter 8}

\section{Baryogenesis in Supersymmetry with a broken $U(1)_{R}$ baryon}

\section{number}

One property of the Universe that any viable theory of particle physics must eventually explain is the asymmetry between matter and antimatter. Several direct and indirect evidences indicate that baryons, which constitute about $5 \%$ of the mass of the Universe [150], are far more abundant than antibaryons [151]. One direct evidence is the observed ratio of antiprotons and protons in cosmic rays of $10^{-4}$, which is consistent with the observed antiprotons simply being secondary products of collisions in the interstellar medium. An indirect evidence is the insufficient observation of several secondary products associated with matter-antimatter annihilation, namely gamma rays, which would be abundant if the Universe contained large quantities of antimatter.

The generation of the imbalance between matter and antimatter in the observed Universe is known as baryogenesis. The necessary elements for this process to take 
place are encoded in the so-called Sakharov conditions [152],

- Baryon number violation,

- $C P$ - and $C$-violation,

- Departure from thermal equilibrium.

The first condition is self-evident. The second condition not being respected would mean that baryons and antibaryons are produced at the same rate and therefore that no net asymmetry is produced. The third condition needs to be satisfied or else CPT invariance would insure compensation between processes increasing and decreasing baryon number [153].

Interestingly, the Standard Model satisfies all three Sakharov conditions. Indeed, it was mentioned in Chapter 2 that baryon number can be violated by nonperturbative effects and that weak interactions do not respect $C$ - and $C P$-invariance. Departure from equilibrium can be obtained in a multitude of ways. Most notably, baryogenesis can take place during the electroweak phase transition if said transition is strongly enough first-order $[153,154]$. As the Universe cools down, bubbles in which the expectation value of the Higgs is non-zero begin to form and expand, filling the Universe. The departure from thermal equilibrium is satisfied in the domain walls surrounding the bubbles and weak interactions provide the source of $C$ - and $C P$ violation. As the bubbles expand, baryons and antibaryons that were once outside the bubbles are scattered by the domain walls. The fact that the Higgs vev is spacedependent in the domain walls results in a space-dependent $C P$-asymmetry, which in turn results in different reflection and transmission coefficients for baryons and antibaryons. This generates the necessary baryon number violation and effectively separates particles from antiparticles. Baryons are more likely than antibaryons to 
pass through the domain walls, while antibaryons are more likely to be reflected outside the bubbles and be subjected again to sphaleron effects, which are still important outside the bubbles. This results in a washing out of the antibaryon density.

However, this process simply does not lead to a large enough baryon density [155]. The CP-phase is insufficient [156] and the phase transition is not enough strongly first-order [157-161]. As such, there have been several attempts to extend the Standard Model to explain the baryon relic density.

Most interesting to us is the possibility of generating baryon asymmetry in the MSSM with RPV couplings and a Mini-Split spectrum. This was first proposed in Ref. [162] and further explored in Refs. [163,164]. In this structure, long lived binos decay out of equilibrium via baryon number violating interactions, thereby satisfying the first and third Sakharov conditions. The second condition is satisfied by having complex gauginos masses, which after field redefinition affect the interference between tree-level and loop-level diagrams. The net result is that binos can be made to decay slightly more often to baryons than to antibaryons. Squarks are required to be heavy enough for the binos to decay after they have decoupled and for the decoupling to be early enough to leave a large bino density. The latter is necessary because the asymmetry is typically small and suppresses the resulting baryon density. At the same time, increasing squark masses decreases the bino decay asymmetry and squarks must therefore not be too heavy. The combination of these constraints naturally leads to a Mini-Split spectrum. Binos are chosen over other gauginos as the latter annihilate too efficiently to lead to a sufficient relic density.

A few additional conditions are however required to be satisfied. First, the requirements of the Nanopoulos-Weinberg theorem must be fulfilled [165]. This theorem essentially states that, for a particle to lead to an antisymmetry between matter and 
antimatter in its decay, it must also be able to decay via another channel. As such, Ref. [162] requires either gluinos or winos to be lighter than the bino. In addition, Higgsinos are required to be heavy for the binos to decouple early enough.

As a side note, Ref. [164] also attempts to explain dark matter relic density by including the gravitino. The hope was that, in the fashion of asymmetric dark matter [166], both could be explained at the same time as the baryon and dark matter relic density are of the same order of magnitude. They however find that obtaining both correct relic densities requires the gravitino mass to be tuned.

Analogous works include Refs. [167, 168] which study similar decays but consider Supersymmetry models with an extended field content. The Mini-Split spectrum requirement is then replaced by the need for some of the new couplings to be small. Ref. [155] also studies baryogenesis for supersymmetric models with a $U(1)_{R}$ symmetry, but focuses on the electroweak phase transition. They find that the new superpotential couplings between the adjoints and Higgses can both make the electroweak phase transition stronger and the lightest Higgs heavier.

In this chapter, we study whether Supersymmetry with a broken $U(1)_{R}$ baryon number can lead to successful baryogenesis via bino decay in the presence of a MiniSplit spectrum. We assume the $U(1)_{R}$ symmetry is broken in the gaugino masses sector by anomaly mediation $[31,32]$ or more generally by Planck scale suppressed operators. More specifically, we concentrate on the case where only binos are light. The Majorana masses of gauginos and adjoints serve as the source of baryon number violation and split the Dirac bino into two Majorana binos. The presence of a $U D D$ term in the superpotential allows the binos to decay to quarks. The heaviest bino can then exhibit baryon asymmetry in its decay, as the presence of the lighter bino automatically satisfies the requirements of the Weinberg-Nanopoulos theorem. 
In addition, the extended Higgs sector allows for lighter Higgsinos than would be permitted in the MSSM. We find that this mechanism can lead to the correct baryon density, but that it unfortunately requires the $U(1)_{R}$ symmetry to be badly broken.

This chapter is organized as follow. We first study $U(1)_{R}$ breaking in the gaugino mass sector. The decays that lead to baryogenesis follow. The different annihilation channels that are responsible for maintaining the binos in thermal equilibrium are discussed. The procedure necessary to obtain an estimate of the relic baryon density is then explained. Illustrative results follow. Finally, a few additional remarks are included about the possibility of light gluinos.

\section{1 $U(1)_{R}$ breaking}

If the $U(1)_{R}$ baryon symmetry was exact, there would obviously not be any baryon asymmetry. However, this symmetry is broken once anomaly mediation is taken into account and could otherwise be broken by Planck scale suppressed operators, as gravity is expected to break any global symmetry. The effect of this breaking that is the most relevant to us is the introduction of Majorana masses for the gauginos and adjoints. Their presence alone is enough to satisfy the first Sakharov condition.

The Majorana masses modify the mass eigenstate structure of the gauginos. The mass Lagrangian of the binos becomes

$$
\mathcal{L}_{\text {masses }}=-\frac{1}{2}\left(\begin{array}{cc}
\tilde{B} & \tilde{S}
\end{array}\right)\left(\begin{array}{cc}
M_{1} & M_{1}^{D} \\
M_{1}^{D} & \rho_{1}
\end{array}\right)\left(\begin{array}{c}
\tilde{B} \\
\tilde{S}
\end{array}\right)+\text { h.c. }
$$

where $\tilde{B}$ represents the bino, $\tilde{S}$ the singlino, $M_{1}^{D}$ the Dirac mass, $M_{1}$ the bino Majorana mass and $\rho_{1}$ the singlino Majorana mass. The Majorana masses cause the Dirac bino to split into two Majorana particles of different masses. We label the lightest 
one by $\chi_{1}^{B}$ and the heaviest by $\chi_{2}^{B}$. We refer to their masses as $m_{1}$ and $m_{2}$ respectively. Making the ratio of $m_{1} / m_{2}$ differ considerably from one requires either $M_{1}$ or $\rho_{1}$ to be at least comparable to $M_{1}^{D}$. The bino and the singlino will then be a linear combination of mass eigenstates of the form

$$
\begin{gathered}
\tilde{B}=a_{1} \chi_{1}^{B}+a_{2} \chi_{2}^{B}, \\
\tilde{S}=b_{1} \chi_{1}^{B}+b_{2} \chi_{2}^{B} .
\end{gathered}
$$

The mixing parameters are generally complex and are responsible for satisfying the second Sakharov condition. For convenience, we refer to $\chi_{1}^{B}$ and $\chi_{2}^{B}$ as binos when the context is clear.

The baryon asymmetry that we obtain in the next section will be proportional to $\sin \phi$ which is defined by

$$
\sin \phi=\frac{\operatorname{Im}\left\{a_{1}^{* 2} a_{2}^{2}\right\}}{\left|a_{1} a_{2}\right|^{2}}
$$

As will be explained more carefully, this term is zero when either $M_{1}$ or $\rho_{1}$ is zero. The combination of this and the mass splitting being inversely proportional to $M_{1}^{D}$ will result in an asymmetry that is strongly suppressed if either $M_{1}$ or $\rho_{1}$ is much smaller than $M_{1}^{D}$.

One important point to mention is that there are several other $U(1)_{R}$ breaking terms that could potentially affect our results. First, A-terms could be introduced, but their effects are typically suppressed by the scalar masses which are assumed large in Mini-Split. Even if they were important, they would not spoil any mechanism and could in fact be used for generating baryon asymmetry. Second, the $\mu$-term of the MSSM could reappear in the superpotential. As will be further discussed in the next section, this would spoil the mechanism that allows for the Higgsinos to be lighter than in the model of Ref. [162]. The $\mu$-term can however be naturally small as it is a 
coefficient in the superpotential. Finally, the most dangerous possibility is soft-terms that mix Higgses such as $H_{u} R_{d}+$ h.c. and $H_{u}^{\dagger} R_{u}+$ h.c.. These terms can lead to the lightest Higgs containing parts of $R_{u}$ and $R_{d}$, which would also reintroduce the need for heavy Higgsinos. This effect can however be suppressed by $R_{u}$ and $R_{d}$ having large soft masses, which we assume from now on to be the case. The only $U(1)_{R}$ breaking that we consider is then the Majorana masses. One property of anomaly mediation worth mentioning is that the problematic terms are either not generated or are suppressed.

\subsection{Baryon asymmetry}

In this section, we explain how the third and final Sakharov condition is satisfied by having $\chi_{2}^{B}$ decay out of equilibrium via baryon number breaking interactions. We first explain the assumptions that must be made concerning the parameter space and then provide the relevant decay widths and resulting baryon asymmetry.

\subsubsection{Assumptions on the parameter space}

Because of the $U D D$ term in the superpotential, the binos will decay via baryon number breaking interactions such as the one shown in Fig. 8.1. In addition, the Nanopoulos-Weinberg theorem requires that $\chi_{2}^{B}$ be able to decay via channels that do not break baryon number. This is automatically realized as gauge interactions allow $\chi_{2}^{B}$ to decay to $\chi_{1}^{B}$ and a pair of quarks, as seen in Fig. 8.2. However, it is necessary for the baryon number breaking decays to dominate. Else, the baryon asymmetry will be suppressed by a small branching ratio. This will require some of the $\lambda_{i j k}^{\prime \prime}$ to be of $\mathcal{O}(0.1)$ or more. This is generally only possible for a few of them [24], though the fact that we deal with a Mini-Split spectrum relaxes the constraints on 


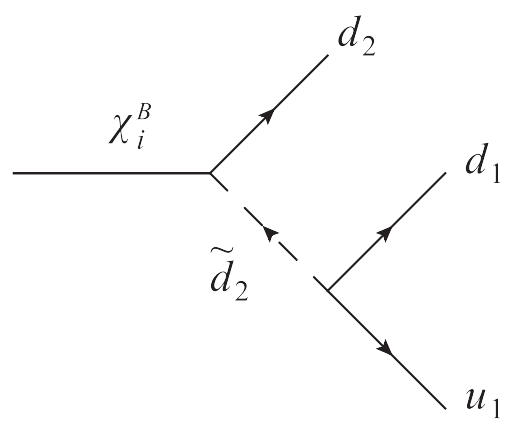

Figure 8.1: Tree-level baryon number breaking decay of binos.

several $\lambda_{i j k}^{\prime \prime}$. As such, we assume that a single $\lambda_{i j k}^{\prime \prime}$ is non-zero and refer to it as $\lambda^{\prime \prime}$. Taking into consideration current constraints, $\lambda_{313}^{\prime \prime}$ and $\lambda_{323}^{\prime \prime}$ would be the most likely candidates [24]. The generalization to several non-zero $\lambda_{i j k}^{\prime \prime}$ 's is trivial. We refer to the associated up quark as $u_{1}$ and the associated down quarks as $d_{1}$ and $d_{2}$. We also assume that a single right-handed sdown-type squark is relevant and take it to be $\tilde{d}_{2}$. We label its mass $m_{\tilde{d}_{2}}$. We could also have taken $\tilde{u}_{1}$ to be light. There is nothing in principle that prevents us from doing this, but the possible presence of the rather large top mass inside of loops would obscure the analysis. We therefore do not consider a light $\tilde{u}_{1}$.

We also assume that $\tilde{d}_{2}$ is considerably heavier than the binos. This is necessary for two reasons. First, $\chi_{2}^{B}$ is required to decay after it decouples to avoid washout effects. This is simply stating that the decay must be out of equilibrium to satisfy the Sakharov conditions. We will use this fact to calculate would-be relic number densities of binos which corresponds to what the relic densities would be if the binos were stable. Second, $\chi_{2}^{B}$ is also required to decouple early. This is simply a question that the baryon number density coming from $\chi_{2}^{B}$ decay is several orders of magnitude smaller than the $\chi_{2}^{B}$ would-be number relic density. We define $x=m_{2} / T$ and label the value of $x$ around which the bino decouples by $x_{f}$. A viable baryogenesis will typically require $x_{f}<5$ [162]. 


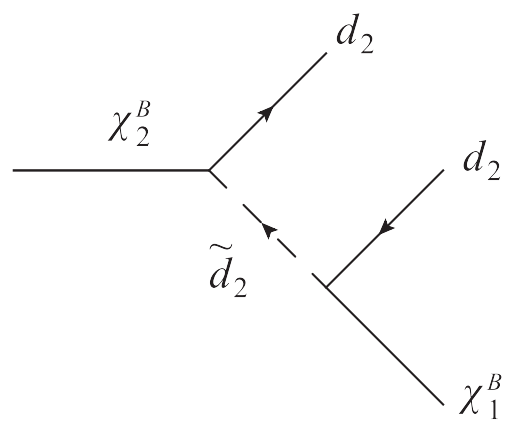

Figure 8.2: Baryon conserving decays of $\chi_{2}^{B}$ to $\chi_{1}^{B}$ and quarks. The diagram with the arrows reversed also exists.

Finally, we assume that winos and gluinos are heavy. We will briefly discuss the case of light gluinos in Section 8.6. We also consider Higgsinos to be heavy but not so much as to be irrelevant. This opens a decay channel to Higgses.

\subsubsection{Decay widths}

We now proceed to list the decay widths of $\chi_{1}^{B}$ and $\chi_{2}^{B}$. All quark masses are neglected.

\section{Baryon breaking decay}

The leading order diagram for baryon breaking decay is shown in Fig. 8.1 and corresponds to a decay width of

$$
\Gamma_{\chi_{i}^{B} \rightarrow u_{1} d_{1} d_{2}}=\frac{g^{\prime 2} Y_{D}^{2}\left|a_{i} \lambda^{\prime \prime}\right|^{2}}{512 \pi^{3}} \frac{m_{i}^{5}}{m_{\tilde{d}_{2}}^{4}}
$$

where $Y_{D}=1 / 3$ is the weak hypercharge of $d_{2}$. 


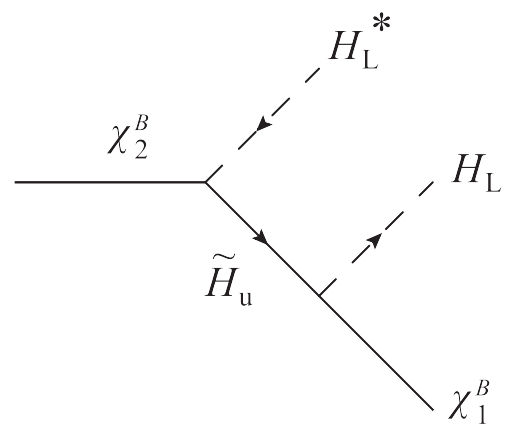

Figure 8.3: Baryon conserving decays of $\chi_{2}^{B}$ to $\chi_{1}^{B}$ and Higgses. Other diagrams exist.

\section{Decay of $\chi_{2}^{B}$ to $\chi_{1}^{B}$ and quarks}

The baryon conserving decay of $\chi_{2}^{B}$ to $d_{2}, \bar{d}_{2}$ and $\chi_{1}^{B}$ is shown in Fig. 8.2. It corresponds to a decay width of

$$
\Gamma_{\chi_{2}^{B} \rightarrow \chi_{1}^{B} d_{2} \bar{d}_{2}}=\frac{g^{\prime 4} Y_{D}^{4}}{256 \pi^{3}}\left[\left|a_{1} a_{2}\right|^{2} f\left(\frac{m_{1}}{m_{2}}\right)+2 \operatorname{Re}\left\{a_{1}^{2} a_{2}^{* 2}\right\} \frac{m_{1}}{m_{2}} g\left(\frac{m_{1}}{m_{2}}\right)\right] \frac{m_{2}^{5}}{m_{\tilde{d}_{2}}^{4}},
$$

where

$$
\begin{aligned}
& f(x)=\left(1-8 x^{2}-12 x^{4} \ln x^{2}+8 x^{6}-x^{8}\right) \theta(1-x), \\
& g(x)=\left(1+9 x^{2}+6 x^{2}\left(1+x^{2}\right) \ln x^{2}-9 x^{4}-x^{6}\right) \theta(1-x) .
\end{aligned}
$$

\section{Decay of $\chi_{2}^{B}$ to $\chi_{1}^{B}$ and Higgses}

An example of decay of $\chi_{2}^{B}$ to $\chi_{1}^{B}$ and two Higgses is shown in Fig. 8.3. This decay leads to the only width that is only suppressed by two powers of a superpartner mass. Other decay processes are instead suppressed by four. As such, the Higgsinos are in general required to be considerably heavier than the scalars. The masses of the Higgs doublets are then approximately given by

$$
\mathcal{L}_{\text {masses }}=-\left(\begin{array}{cc}
H_{u}^{\dagger} & \tilde{H}_{d}^{\dagger}
\end{array}\right)\left(\begin{array}{cc}
\mu_{u}^{2} & B_{\mu} \\
B_{\mu} & \mu_{d}^{2}
\end{array}\right)\left(\begin{array}{c}
H_{u} \\
\tilde{H}_{d}
\end{array}\right)
$$


where $\tilde{H}_{d}=i \sigma^{2} H_{d}^{*}$ and where we assumed that $\mu_{u}, \mu_{d}$ and $B_{\mu}$ are real. Requiring one of the Higgses to be light necessitates $B_{\mu}^{2}=\mu_{u}^{2} \mu_{d}^{2}$. The resulting light Higgs $H_{L}$ is then given by

$$
H_{L}=\frac{1}{\sqrt{\mu_{u}^{2}+\mu_{d}^{2}}}\left(\mu_{d} H_{u}+\mu_{u} \tilde{H}_{d}\right) .
$$

In this limit, the corresponding decay width is given by

$$
\Gamma_{\chi_{2}^{B} \rightarrow \chi_{1}^{B} H_{L} H_{L}^{*}}=\frac{1}{768 \pi^{2}}\left[\left|C_{12}\right|^{2} u\left(\frac{m_{1}}{m_{2}}\right)+3 \operatorname{Re}\left\{C_{12}^{2}\right\} \frac{m_{1}}{m_{2}} v\left(\frac{m_{1}}{m_{2}}\right)\right] m_{2}^{3}
$$

where

$$
u(x)=\left(1-x^{2}\right)^{3} \theta(1-x), \quad v(x)=\left(1+2 x^{2} \ln x^{2}-x^{4}\right) \theta(1-x),
$$

and

$$
C_{i j}=\frac{g^{\prime}}{\mu_{u}^{2}+\mu_{d}^{2}}\left(\lambda_{u}^{s} \frac{\mu_{d}^{2}}{\mu_{u}}-\lambda_{d}^{s} \frac{\mu_{u}^{2}}{\mu_{d}}\right)\left(a_{i} b_{j}+a_{j} b_{i}\right)
$$

A similar result exists for Mini-Split leptogenesis. In this case, the wino is required to be lighter than the bino for leptogenesis to occur. The bino can then decay to the wino and Higgses with a decay width of [162]

$$
\Gamma_{\tilde{B} \rightarrow \tilde{W} H_{L} H_{L}^{*}}^{\mathrm{SM}}=\frac{\left(Y_{H} g_{1} g_{2}\right)^{2}}{384 \pi^{3}} \frac{M_{1}^{3}}{\mu^{2}}
$$

where $Y_{H}=1 / 2$ is the weak hypercharge of the Higgs doublet. The main difference of Eq. (8.9) is the presence of $\lambda_{u}^{s}$ and $\lambda_{d}^{s}$, which is an effect of the extended Higgs sector. Being coefficients in the superpotential, $\lambda_{u}^{s}$ and $\lambda_{d}^{s}$ can naturally be small and the Higgsinos are not required to be as heavy as in the MSSM. As was alluded to earlier, this mechanism can however be spoiled by the presence of either a $\mu$-term or soft-terms like $H_{u} R_{d}+$ h.c.. These terms would allow for diagrams that do not require 
$\lambda_{u}^{s}$ and $\lambda_{d}^{s}$. That is why we needed to make assumptions to limit their effects.

We note that the width of this decay channel does not go to zero when $\lambda_{u}^{s}$ and $\lambda_{d}^{s}$ are zero, but that it would instead be suppressed by higher powers of $\mu_{u}$ and $\mu_{d}$. We also note that this result is not exact as the decay will typically take place after the electroweak phase transition. The degrees of freedom involved will not be the same and the exact expression depends on the precise details of the scalar sector. As we are more interested in a proof of principle, we will be satisfied with this result.

\section{Decay of $\chi_{2}^{B}$ to $\chi_{1}^{B}$ and a photon}

Finally, $\chi_{2}^{B}$ can also decay to $\chi_{1}^{B}$ and a photon as shown in Fig. 8.4. The decay width is

$$
\begin{aligned}
\Gamma_{\chi_{2}^{B} \rightarrow \chi_{1}^{B} \gamma}=\frac{e^{2} g^{\prime 4} Y_{D}^{4}}{8192 \pi^{5}} & {\left[\left|a_{1} a_{2}\right|^{2}+2 \operatorname{Re}\left\{a_{1}^{2} a_{2}^{* 2}\right\} \frac{m_{1} m_{2}}{m_{1}^{2}+m_{2}^{2}}\right] } \\
& \left(1+\left(\frac{m_{1}}{m_{2}}\right)^{2}\right)\left(1-\left(\frac{m_{1}}{m_{2}}\right)^{2}\right)^{3} \frac{m_{2}^{5}}{m_{\tilde{d}_{2}}^{4}}
\end{aligned}
$$

which is negligible. Note that it was assumed that the decay takes place after the electroweak phase transition, which will turn out to be case in most of the successful region of parameter space. If the decay were to take place before the phase transition, it would instead involve a $B$ boson. The answer would change slightly but would still remain negligible. The decay to a $Z$ boson is also negligible.

\subsubsection{Net decay width to baryons}

The difference between the decay width of $\chi_{2}^{B}$ to baryons and antibaryons comes from the interference between the tree-level diagram of Fig. 8.1 and the loop diagram of Fig. 8.5. Other diagrams exist, but either do not lead to any baryon asymmetry or require a dangerous amount of flavour mixing [162]. The net decay width to baryons 


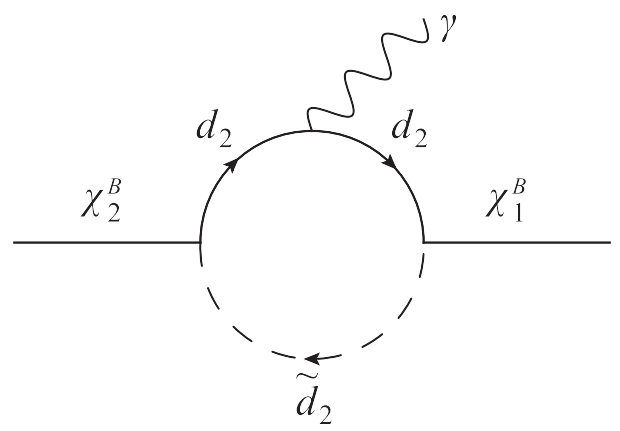

Figure 8.4: Decay of $\chi_{2}^{B}$ to $\chi_{1}^{B}$ and a photon. The photon can also be inserted on the squark line and the arrow flow can be reversed in both cases.

is given by

$$
\Delta \Gamma^{B}=\Gamma_{\chi_{2}^{B} \rightarrow u_{1} d_{1} d_{2}}^{B}-\Gamma_{\chi_{2}^{B} \rightarrow \bar{u}_{1} \bar{d}_{1} \bar{d}_{2}}^{B}=\frac{g^{\prime 4} Y_{D}^{4}\left|a_{1} a_{2} \lambda^{\prime \prime}\right|^{2} \sin \phi}{2048 \pi^{4}} f\left(\frac{m_{1}}{m_{2}}\right)\left(\frac{m_{2}}{m_{\tilde{d}_{2}}}\right)^{6} m_{1} .
$$

We note that this result illustrates the Nanopoulos-Weinberg theorem. The net width $\Delta \Gamma^{B}$ would be zero if $m_{1}>m_{2}$ since $f(x)$ is 0 for $x \geq 1$. This also corresponds to the decay of $\chi_{2}^{B}$ to $\chi_{1}^{B}$ and quarks being forbidden. Obviously, this is not a problem because $m_{1}<m_{2}$ by assumption, but it does show that the decay of $\chi_{1}^{B}$ does not lead to baryon asymmetry.

We can now better understand why both Majorana masses need to be non-zero. First, consider the case of $\rho_{1}$ set to zero. The interference term between the diagrams of Figs. 8.1 and 8.5 can be factorized as a function of the coupling constants times a function depending only on the kinematics. To obtain a net baryon asymmetry, both of these functions must be complex [169]. ${ }^{1}$ First, the kinematic function is complex because the loop diagram in Fig. 8.5 can be cut to obtain the diagram in Fig. 8.1 (see Ref. [170]). Second, the phase of $M_{1}$ can be reabsorbed by a field redefinition of $\tilde{B}$. This effectively makes $g^{\prime}$ complex. It also transfers a phase to $M_{1}^{D}$, which can

\footnotetext{
${ }^{1}$ It is assumed that the fields have been redefined such that their masses are real.
} 


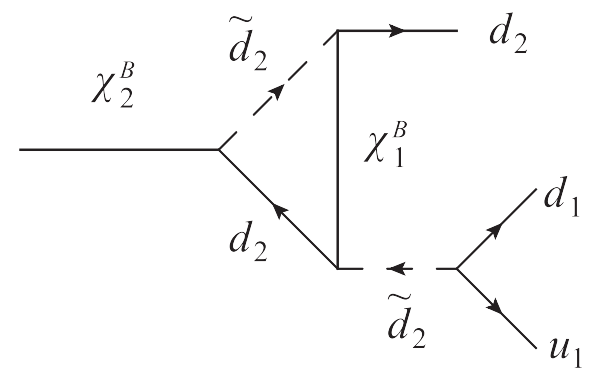

Figure 8.5: Loop decay of $\chi_{2}^{B}$ via virtual $\chi_{1}^{B}$.

then be removed by a field redefinition of $\tilde{S}$. Since none of the couplings associated with this field are involved in these diagrams, the phase will only appear in $g^{\prime}$. Since $g^{\prime}$ appears as $\left|g^{\prime}\right|^{4}$ in the interference term, this would not lead to any asymmetry. The procedure obviously breaks down when $\rho_{1}$ is non-zero, as the field redefinition we did would lead to a complex $\rho_{1}$. A similar argument holds for $M_{1}$.

Finally, the resulting asymmetry for the decay of $\chi_{2}^{B}$ is given by

$$
\epsilon_{C P}=\frac{\Delta \Gamma^{B}}{\Gamma_{\chi_{2}^{B}}^{\text {total }}}=\frac{\Delta \Gamma^{B}}{\Gamma_{\chi_{2}^{B} \rightarrow u_{1} d_{1} d_{2}}+\Gamma_{\chi_{2}^{B} \rightarrow \bar{u}_{1} \bar{d}_{1} \bar{d}_{2}}+\Gamma_{\chi_{2}^{B} \rightarrow \chi_{1}^{B} d_{2} \bar{d}_{2}}+\Gamma_{\chi_{2}^{B} \rightarrow \chi_{1}^{B} H_{L} H_{L}^{*}}},
$$

where we neglected the decays to gauge bosons.

\subsection{Annihilation and conversion cross sections}

We now proceed to calculate the annihilation and conversion cross sections of binos. These enter the Boltzmann equations which will be used to calculate the would-be relic density of the binos. The two most important interactions are those with Higgses and quarks. 
(a)

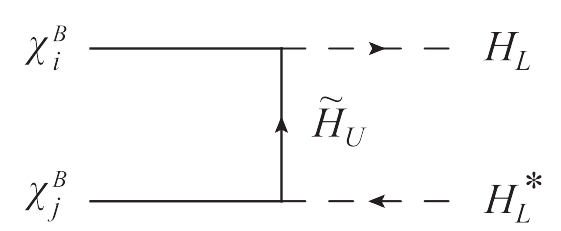

(b)

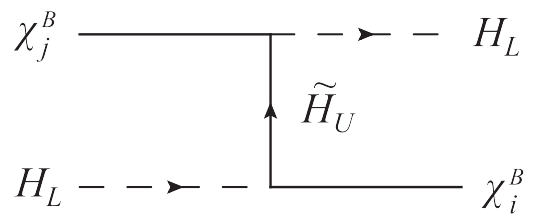

Figure 8.6: (a) Annihilation to Higgses. There also exist other diagrams. (b) Example of bino conversion via Higgs scattering. There also exist other diagrams.

\subsubsection{Interactions with Higgses}

An example of annihilation to two Higgses is shown in Fig. 8.6a. The total cross section is given by

$$
\sigma_{\chi_{i}^{B} \chi_{j}^{B} \rightarrow H_{L} H_{L}^{*}}(s)=\frac{1}{32 \pi} \frac{\left|C_{i j}\right|^{2}\left(s-m_{i}^{2}-m_{j}^{2}\right)-2 \operatorname{Re}\left\{C_{i j}^{2}\right\} m_{i} m_{j}}{\sqrt{\left(s-\left(m_{i}+m_{j}\right)^{2}\right)\left(s-\left(m_{i}-m_{j}\right)^{2}\right)}},
$$

where $\sqrt{s}$ is the center of mass energy. As we will be interested in binos of around a $\mathrm{TeV}$ or heavier and that decouple at very small $x$, freeze-out will typically take place before the electroweak phase transition. As such, $H_{L}$ is treated as a complex scalar doublet for calculating relic densities, i.e. no particle has been "eaten" yet by gauge bosons.

In addition to annihilation, one must also take into account conversion via scattering. An example is shown in Fig. 8.6b. The associated cross section is

$$
\sigma_{\chi_{j}^{B} H_{L} \rightarrow \chi_{i}^{B} H_{L}}=\frac{1}{64 \pi s}\left(\frac{s-m_{i}^{2}}{s-m_{j}^{2}}\right)\left[\left|C_{i j}\right|^{2} \frac{\left(s+m_{i}^{2}\right)\left(s+m_{j}^{2}\right)}{s}+4 m_{i} m_{j} \operatorname{Re}\left\{C_{i j}^{2}\right\}\right] .
$$

Note that this result is averaged over the two possible Higgses and their conjugates. 
(a)

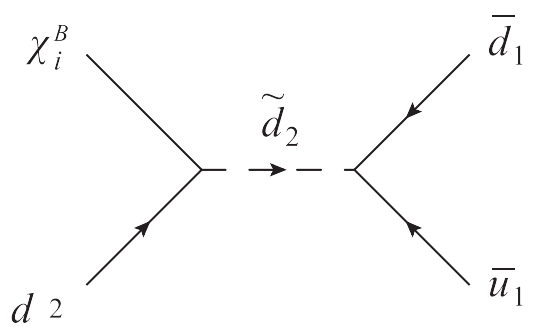

(b)

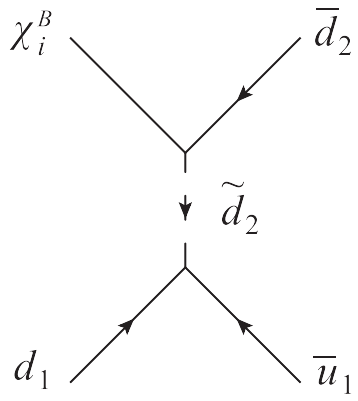

Figure 8.7: (a) Example of $s$-channel annihilation of a single bino. (b) Example of $t$-channel annihilation of a single bino.

\subsubsection{Interactions with quarks}

We now discuss annihilation and conversion via interactions with quarks. These interactions can either conserve baryon number or break it.

For $\lambda^{\prime \prime}$ of $\mathcal{O}(0.1)$ or larger and heavy Higgsinos, baryon number breaking annihilation is expected to dominate because of the large multiplicity and lack of $p$-wave suppression [162]. Examples of these interactions are shown in Fig. 8.7. The cross section is given by

$$
\sigma_{\chi_{i}^{B} q \rightarrow q q}(s)=\frac{g^{\prime 2} Y_{D}^{2}\left|a_{i} \lambda^{\prime \prime}\right|^{2}}{48 \pi m_{\tilde{d}_{2}}^{4}}\left(5 s+m_{i}^{2}\right) .
$$

Other subleading interactions with quarks exist that preserve baryon number. These effects are usually subdominant. Annihilation of binos to quarks is shown in Fig. 8.8a. The associated cross section is

$$
\begin{gathered}
\sigma_{\chi_{i}^{B} \chi_{j}^{B} \rightarrow d_{2} \bar{d}_{2}}(s)=\frac{g^{\prime 4} Y_{D}^{4}}{16 \pi m_{\tilde{d}_{2}}^{4}} \frac{1}{\sqrt{\left(s-\left(m_{i}+m_{j}\right)^{2}\right)\left(s-\left(m_{i}-m_{j}\right)^{2}\right)}} \\
{\left[\left|a_{i} a_{j}\right|^{2}\left(2 s^{2}-\left(m_{i}^{2}+m_{j}^{2}\right) s-\left(m_{i}^{2}-m_{j}^{2}\right)^{2}\right)-6 \operatorname{Re}\left\{a_{i}^{2} a_{j}^{* 2}\right\} m_{i} m_{j} s\right] .}
\end{gathered}
$$

In addition, conversion can take place via the diagram of Fig. 8.8b. The associated 
(a)

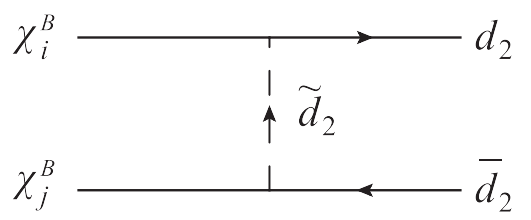

(b)

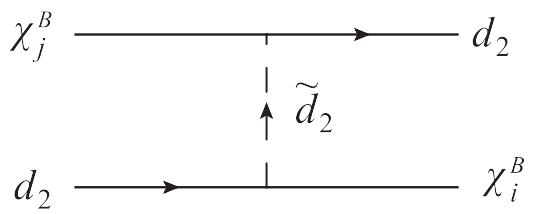

Figure 8.8: (a) Example of bino pair annihilation to $d_{2} \bar{d}_{2}$. There is also a diagram with the incoming lines crossed. (b) Example of bino conversion via scattering off quarks. There is also an $s$-channel diagram.

cross section is

$$
\begin{gathered}
\sigma_{\chi_{j}^{B} d_{2} \rightarrow \chi_{i}^{B} d_{2}}(s)=\frac{g^{\prime 4} Y_{D}^{4}}{96 \pi m_{\tilde{d}_{2}}^{4}} \frac{\left(s-m_{i}^{2}\right)^{2}}{s^{3}}\left[\left|a_{i} a_{j}\right|^{2}\left(8 s^{2}+\left(m_{i}^{2}+m_{j}^{2}\right) s+2 m_{i}^{2} m_{j}^{2}\right)\right. \\
\left.+6 \operatorname{Re}\left\{a_{i}^{2} a_{j}^{* 2}\right\} m_{i} m_{j} s\right] .
\end{gathered}
$$

\subsection{Calculation of $\Omega_{\Delta B}$}

To obtain estimates of the baryon relic density $\Omega_{\Delta B}$, we calculate would-be relic densities of binos. For this, we use Boltzmann's equation conveniently rewritten as [171] (see also Ref. [164]),

$$
\begin{gathered}
\frac{d Y_{i}}{d x}=-\sqrt{\frac{g_{*} \pi}{45 G}} \frac{m_{2}}{x^{2}}\left[\sum_{j=1}^{2}\left\langle\sigma_{i j} v_{i j}\right\rangle\left(Y_{i} Y_{j}-Y_{i}^{\mathrm{eq}} Y_{j}^{\mathrm{eq}}\right)+\left\langle\sigma_{i X} v_{i X}\right\rangle\left(Y_{i}-Y_{i}^{\mathrm{eq}}\right) Y_{X}^{\mathrm{eq}}\right. \\
\left.\quad-\left\langle\sigma_{j X} v_{j X}\right\rangle\left(Y_{j}-\frac{Y_{j}^{\mathrm{eq}}}{Y_{i}^{\mathrm{eq}}} Y_{i}\right) Y_{X}^{\mathrm{eq}}\right] .
\end{gathered}
$$

The parameter $Y_{i}$ is given by $Y_{i}=n_{i} / s$, where $n_{i}$ is the number density of particle $i$ and $s$ the entropy per comoving volume (not to be confused with the center of mass energy). The parameter $g_{*}$ corresponds to the number of relativistic degrees of freedom. As we deal with particles with masses of the order of a few $\mathrm{TeV}$ and which 
decouple at small $x, g_{*}$ can safely be approximated by a constant $g_{*} \approx 106.75$. The parameter $Y_{i}^{\text {eq }}$ represents the equilibrium value of $Y_{i}$ and is given by [171]

$$
Y_{i}^{\mathrm{eq}}=\frac{45 x^{2}}{4 \pi^{4} g_{*}} g_{i}\left(\frac{m_{i}}{m_{2}}\right)^{2} K_{2}\left(x \frac{m_{i}}{m_{2}}\right)
$$

where $g_{i}$ is the number of degrees of freedom of the particle $i$ and $K_{i}(x)$ is a modified Bessel functions of the second kind. The $\left\langle\sigma_{i j} v_{i j}\right\rangle,\left\langle\sigma_{i X} v_{i X}\right\rangle$ and $\left\langle\sigma_{j X} v_{j X}\right\rangle$ represent thermally averaged cross sections and can be obtained by combining the results of Section 8.3 with the following Eq. [171],

$$
\left\langle\sigma_{i j} v_{i j}\right\rangle=\frac{\int_{\left(m_{i}+m_{j}\right)^{2}}^{\infty} \frac{1}{\sqrt{s}}\left(s-\left(m_{i}+m_{j}\right)^{2}\right)\left(s-\left(m_{i}-m_{j}\right)^{2}\right) K_{1}\left(\frac{\sqrt{s}}{T}\right) \sigma_{i j}(s) d s}{8 T m_{i}^{2} m_{j}^{2} K_{2}\left(\frac{m_{i}}{T}\right) K_{2}\left(\frac{m_{j}}{T}\right)}
$$

where the $i$ and $j$ indices can represent any particle. Annihilation of two binos contributes to $\left\langle\sigma_{i j} v_{i j}\right\rangle$, annihilation of a single bino contributes to $\left\langle\sigma_{i X} v_{i X}\right\rangle$ and conversion contributes to $\left\langle\sigma_{j X} v_{j X}\right\rangle$. Once the would-be relic density is obtained, $\Omega_{\Delta B}$ is approximated by

$$
\Omega_{\Delta B}=\left.\frac{m_{p}}{\left(\rho_{c} / s\right)_{0}} \epsilon_{C P} Y_{2}\right|^{t \rightarrow \infty}
$$

where $m_{p}$ is the mass of the proton and $\left(\rho_{c} / s\right)_{0}$ the current ratio of critical density to entropy density. This is a good approximation as long as the decay temperature of the bino is considerably lower than its freeze-out temperature. Also, note that baryon number breaking interactions that take place before freeze-out can lead to an additional source of baryon asymmetry. This was studied in Ref. [164] and found to be negligible because of washout effects. 


\subsection{Results and constraints}

We now proceed to discuss the relevant constraints and provide a few benchmark plots to illustrate different features. We stress that we do not aim to cover the full parameter space, but to show that it is indeed possible to obtain a sufficient baryon density.

We first make a few simplifying assumptions out of convenience. We relate parameters by setting $\mu_{u}=\mu_{d} \equiv \mu$ and $\lambda_{u}^{s}=-\lambda_{d}^{s} \equiv \lambda^{s}$. We also set $\lambda^{\prime \prime}=0.2$, which is chosen to maximize $\Omega_{\Delta B}$. It is large enough for $\epsilon_{C P}$ not to be suppressed by large decay branching ratios to other channels, while not being so large as to make $B$-breaking scattering with quarks too strong.

Fig. 8.9 shows $\Omega_{\Delta B}$ as a function of $M_{1}$ and $M_{1}^{D}$ for decoupled Higgsinos. The mass $\rho_{1}$ is set to $1 \times \exp (3 i / 4 \pi) \mathrm{TeV}$ and $m_{\tilde{d}_{2}}$ to $50 \mathrm{TeV}$. As can be seen, it is possible to obtain a sufficient baryon relic density, but it requires the $U(1)_{R}$ breaking to be large. We see that $\Omega_{\Delta B}$ peaks in a region where $\chi_{2}^{B}$ is very close to being a pure singlino. In this limit, $\chi_{2}^{B}$ can easily decouple early when the Higgsinos are heavy as interactions with quarks are suppressed. It is then not necessary to have the squarks as heavy for it to decouple early and $\epsilon_{C P}$ doesn't need to be as suppressed. We note that $\Omega_{\Delta B}$ is optimized when $m_{1} / m_{2}$ is close to 0.25 . This corresponds to the maximum of $x f(x)$, which controls the asymmetry (see Eq. (8.15)).

Fig. 8.10 shows $\Omega_{\Delta B}$ as a function of $\mu / \lambda^{s}$ and $m_{\tilde{d}_{2}}$. The masses $M_{1}^{D}, M_{1}$ and $\rho_{1}$ are set respectively to $0.02 \mathrm{TeV}, 0.25 \mathrm{TeV}$ and $1 \times \exp (3 i / 4 \pi) \mathrm{TeV}$. Obviously, Higgsinos can be made lighter by taking $\lambda^{s}$ small but eventually the subleading corrections inversely proportional to $\mu^{4}$ would become non-negligible. In addition, this shows that the requirement of correct $\Omega_{\Delta B}$ indeed leads to a Mini-Split spectrum.

A few additional constraints are also taken into account. The first one concerns 


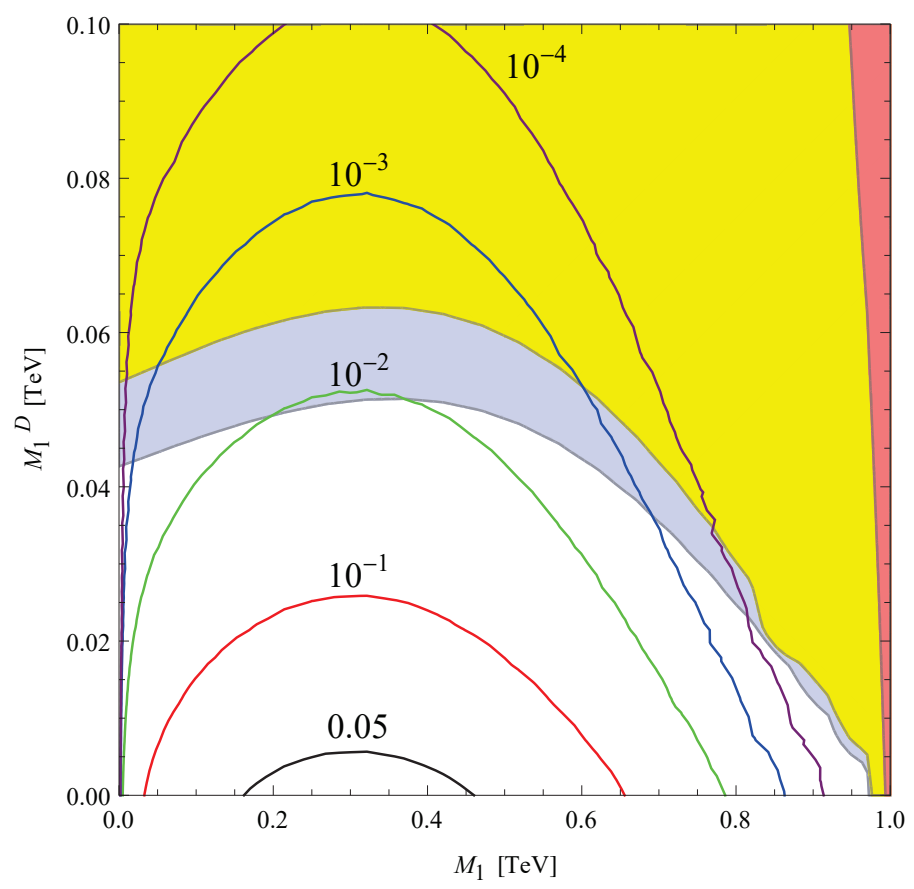

Figure 8.9: Contour plots of constant $\Omega_{\Delta B}$. The blue region corresponds to $\chi_{2}^{B}$ decaying before electroweak phase transition. The yellow region corresponds to $\chi_{2}^{B}$ decaying before freeze-out. The pink region is excluded by washout.

washout. The relevant washout processes are carefully discussed in Ref. [167]. They include inverse decay via on-shell squark and $u_{1} d_{1} d_{2} \rightarrow \bar{u}_{1} \bar{d}_{1} \bar{d}_{2}$ just to name a few. The end result is that they are suppressed as long as the decay temperature of $\chi_{2}^{B}$ is lower than its mass. This excluded region corresponds to the pink region.

The second constraint to take into account is for $\chi_{2}^{B}$ to decay after its freezeout. This constraint is not absolute, as decays that take place slightly before still lead to a relic baryon density, albeit suppressed. We estimate the freeze-out temperature of $\chi_{2}^{B}$ by taking its relic number density, setting it equal to the equilibrium density and solving for $x$. This defines a freeze-out temperature. We then include in the figures the region where $\chi_{2}^{B}$ decays before freeze-out in yellow. Generally speaking, this constraint is far more important than washout and completely overshadows it. This was to be expected. 


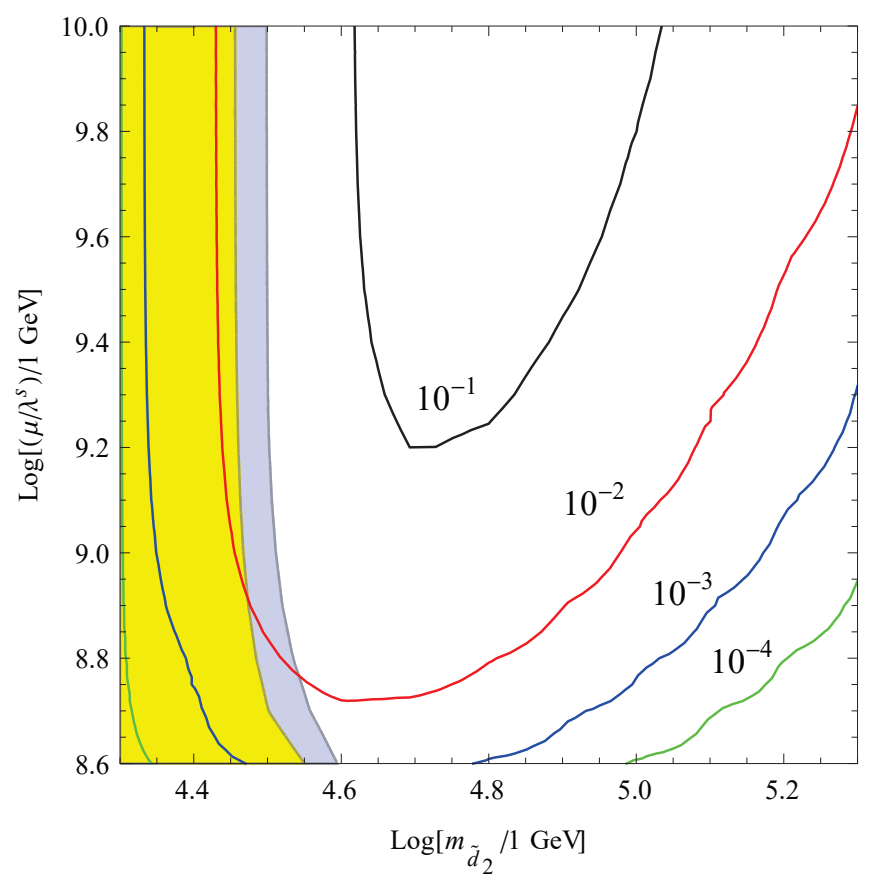

Figure 8.10: Contour plots of constant $\Omega_{\Delta B}$. The blue region corresponds to $\chi_{2}^{B}$ decaying before electroweak phase transition. The yellow region corresponds to $\chi_{2}^{B}$ decaying before freeze-out.

Finally, if $\chi_{2}^{B}$ were to decay before the electroweak phase transition, some of the baryon relic density would be converted away by sphaleron effects. This would reduce the baryon relic density by a factor of $28 / 79$, which is sizable but does not change the qualitative features [172]. It also corresponds in our plots to a region that does not produce enough baryon relic density. We take the electroweak phase transition to take place at $100 \mathrm{GeV}$. We show the regions where the decay of $\chi_{2}^{B}$ takes place before the electroweak phase transition in blue.

Another effect to consider is the possibility of entropy dilution. Ref. [164] studied this and found that it is only relevant for very large scalar masses where the bino decouples while still relativistic. This would lead to a suppression of $\Omega_{\Delta B}$ by a 
dilution factor of $[164,173]$

$$
\xi_{s}=\operatorname{Max}\left[1,1.8 g_{*}^{1 / 4} \frac{Y_{2}\left(x_{f . o .}\right) m_{2}}{\sqrt{\Gamma_{\chi_{2}^{B}}^{\text {total }} M_{\mathrm{Pl}}}}\right],
$$

where $\Gamma_{\chi_{2}^{B}}^{\text {total }}$ is the value of $Y_{2}$ when $\chi_{2}^{B}$ freezes-out and $M_{\mathrm{Pl}}$ the Planck scale. We have verified that this factor is simply one over all the region shown in our plots.

\subsection{Inclusion of light gluinos}

As was made clear in the last section, getting the correct baryon relic density is possible, but requires a very large $U(1)_{R}$ breaking. One possibility to alleviate this problem is to allow for light gluinos. These can indeed contribute to baryon asymmetry by running in loop diagrams similar to Fig. 8.5. There will now be four diagrams, corresponding to each combination of incoming bino and gluino running in the loop. All decay widths can be obtained in a similar way to Section 8.2.

The procedure is however far more complex, as interactions with gluinos are now important and as such the number density of gluinos must be tracked. See Ref. [164] for a thorough treatment in the context of the MSSM. We therefore leave this for future work.

However, it is clear that the presence of light gluinos will not improve the results much. Indeed, strong coupling might lead to a larger $\epsilon_{C P}$, but they also lead to larger interaction cross sections which lead to decoupling at lower temperature and thus a lower would-be relic density of binos. In addition, Fig. 8.9 shows that the drop in $\Omega_{\Delta B}$ is very abrupt once the ratio of Majorana masses to Dirac masses becomes small. As such, a gluino LSP would still require the $U(1)_{R}$ symmetry to be broken badly.

One advantage of gluinos however is that there would be no need for singlino 
or octino masses. The argument of Section 8.2.3 simply does not apply when two different types of gauginos are involved. This would make it easier to justify this mechanism in term of anomaly mediation, where the masses of adjoint fermions are not expected to be large. 


\section{Chapter 9}

\section{Summary}

Despite all of its successes, the Standard Model is still plagued by several issues, both experimental and theoretical. Chief among these is the hierarchy problem, the instability of the electroweak scale under radiative corrections. The most famous attempt at solving this problem is Supersymmetry, which postulates a partner of different spin for every particle of the Standard Model. In addition to solving the hierarchy problem, Supersymmetry provides a dark matter candidate and leads to gauge coupling unification. For Supersymmetry to be natural however, some superpartners are required to be light, which can be difficult considering current experimental constraints. Hence, the concept of neutral naturalness was proposed, where stabilizing partners are not charged under the Standard Model gauge groups and could therefore more easily avoid detection.

In light of these considerations, the purpose of this thesis was to study possible avenues in Supersymmetry and naturalness. More precisely, we investigated four specific models.

In Chapter 4, we presented a new iteration of the Twin Higgs model. The novelty was that the $\mathbb{Z}_{2}$ breaking was spontaneous instead of explicit. This was done 
by expanding the Higgs sector to include two fundamentals of $S U(4)$. One of them preserves $\mathbb{Z}_{2}$, while the other breaks it spontaneously. A bilinear term involving both fields transmits the $\mathbb{Z}_{2}$ breaking from the broken to the unbroken sector. In addition to being theoretically more attractive, the model has the advantages of reducing the necessary tuning and making many mirror partners heavier.

Chapter 5 focused on a class of Supersymmetry models called Mini-Split. In such models, the gauginos are relatively light, while the scalars are a few orders of magnitude heavier. The presence of this small hierarchy deflects the gaugino masses from their usual expressions in gauge and anomaly mediation. More precisely, we studied the current constraints on deflected anomaly and gauge mediation, as well as their prospect for Run-II of the LHC and at a future $100 \mathrm{TeV}$ collider. The Higgsinos are assumed around the scalar scale and a lighter third generation of squarks is assumed. The resulting constraints are summarized in Section 5.2.

Chapter 7 concerned supersymmetric models with a $U(1)_{R}$ symmetry associated with the lepton number. One of the sneutrinos acquires a vev and provides mass to down-type quarks and charged leptons. However, there are severe constraints from electroweak precision tests. It was the purpose of this chapter to synthesize them. The region of parameter space still available can be found in Section 7.3.

Chapter 8 concentrated on the case where $U(1)_{R}$ charges are instead matched to the baryon number. This symmetry is expected to be broken by anomaly mediation or by Planck scale suppressed operators. This naturally provides all the necessary elements for baryogenesis. The correct baryon density can be obtained in the presence of a Mini-Split spectrum, but requires the $U(1)_{R}$ symmetry to be badly broken. A consequence of the extended Higgs sector is that Higgsinos are not required to be as heavy as in the MSSM. Benchmark plots are shown in Section 8.5. 


\section{Appendix A}

\section{Conventions}

The conventions and notations used in this thesis are summarized here for convenience. The Minkowski metric is taken in the mostly minus convention, i.e.

$$
\eta^{\mu \nu}=\left(\begin{array}{cccc}
1 & 0 & 0 & 0 \\
0 & -1 & 0 & 0 \\
0 & 0 & -1 & 0 \\
0 & 0 & 0 & -1
\end{array}\right)
$$

The Pauli matrices are given by

$$
\sigma^{1}=\left(\begin{array}{cc}
0 & 1 \\
1 & 0
\end{array}\right), \quad \sigma^{2}=\left(\begin{array}{cc}
0 & -i \\
i & 0
\end{array}\right), \quad \sigma^{3}=\left(\begin{array}{cc}
1 & 0 \\
0 & -1
\end{array}\right)
$$

They are often arranged as a three-vector

$$
\vec{\sigma}=\left(\sigma^{1}, \sigma^{2}, \sigma^{3}\right) .
$$


This in turn can be used to arrange the Pauli matrices in four-vectors

$$
\sigma^{\mu}=(\mathbf{1}, \vec{\sigma}), \quad \bar{\sigma}^{\mu}=(\mathbf{1},-\vec{\sigma}) .
$$

With these, we can define

$$
\sigma^{\mu \nu}=\frac{i}{4}\left(\sigma^{\mu} \bar{\sigma}^{\nu}-\sigma^{\nu} \bar{\sigma}^{\mu}\right), \quad \bar{\sigma}^{\mu \nu}=\frac{i}{4}\left(\bar{\sigma}^{\mu} \sigma^{\nu}-\bar{\sigma}^{\nu} \sigma^{\mu}\right)
$$

The Dirac matrices are taken as

$$
\gamma^{0}=\left(\begin{array}{cc}
0 & \mathbf{1} \\
\mathbf{1} & 0
\end{array}\right), \quad \vec{\gamma}=\left(\begin{array}{cc}
0 & -\vec{\sigma} \\
\vec{\sigma} & 0
\end{array}\right)
$$

and can be arranged in a four-vector

$$
\gamma^{\mu}=\left(\gamma^{0}, \vec{\gamma}\right)
$$

The Dirac matrices satisfy the Clifford algebra

$$
\left\{\gamma^{\mu}, \gamma^{\nu}\right\}=2 \eta^{\mu \nu}
$$

With this relation, it is easy to verify that the quantity

$$
M^{\mu \nu}=\frac{i}{4}\left[\gamma^{\mu}, \gamma^{\nu}\right]
$$

satisfies the Lorentz algebra (see Eq. (3.2)). A field that transforms under the Lorentz group with a representation of $M^{\mu \nu}$ as defined in Eq. (A.9) is referred to as a Dirac spinor. With the conventions used, $M^{\mu \nu}$ is block-diagonal. A Dirac spinor $\psi$ can 
therefore be written in terms of two two-component spinors that do not mix under boosts and rotations

$$
\psi=\left(\begin{array}{c}
\eta_{R} \\
\chi_{L}
\end{array}\right)
$$

where $\eta_{R}$ and $\chi_{L}$ are referred to as right-handed and left-handed Weyl spinors respectively. The treatment of Weyl spinors is simplified by the van der Waerden notation, which is discussed in more detail in Appendix C. A 4-component spinor $\psi$ formed from a left-handed spinor $\chi_{L}$ and $i \sigma^{2} \chi_{L}^{*}$ as its right-handed part is referred to as Majorana and respects

$$
\psi^{c}=C \bar{\psi}^{T}=\psi
$$

where $C=-i \gamma^{2} \gamma^{0}$ is the charge conjugation matrix and

$$
\bar{\psi}=\psi^{\dagger} \gamma^{0}
$$

Finally, $\gamma^{5}$ is defined via

$$
\gamma^{5}=i \gamma^{0} \gamma^{1} \gamma^{2} \gamma^{3}=\left(\begin{array}{cc}
\mathbf{1} & 0 \\
0 & -\mathbf{1}
\end{array}\right)
$$

and the left-handed and right-handed projectors $P_{L}$ and $P_{R}$ are defined by

$$
P_{L}=\frac{1}{2}\left(\mathbf{1}-\gamma^{5}\right), \quad P_{R}=\frac{1}{2}\left(\mathbf{1}+\gamma^{5}\right)
$$

Unless stated otherwise, repeated indices are assumed summed. 


\section{Appendix B}

\section{Grassmann variables}

This appendix gives a short introduction to Grassmann variables. Their main prop-

erty is that they anticommute instead of commuting. Assume two Grassmann variables $\theta_{1}$ and $\theta_{2}$. Then,

$$
\left\{\theta_{1}, \theta_{1}\right\}=\left\{\theta_{2}, \theta_{2}\right\}=\left\{\theta_{1}, \theta_{2}\right\}=0
$$

The derivative with respect to a Grassmann variable is for the most part similar to a derivative with respect to a complex variable, i.e.

$$
\frac{\partial \theta_{1}}{\partial \theta_{1}}=\frac{\partial \theta_{2}}{\partial \theta_{2}}=1, \quad \frac{\partial \theta_{1}}{\partial \theta_{2}}=\frac{\partial \theta_{2}}{\partial \theta_{1}}=0 .
$$

The only difference is that derivatives also anticommute with each other and with other Grassmann variables. For example,

$$
\frac{\partial}{\partial \theta_{1}}\left(\theta_{2} \theta_{1}\right)=\frac{\partial \theta_{2}}{\partial \theta_{1}} \theta_{1}-\theta_{2} \frac{\partial \theta_{1}}{\partial \theta_{1}}=-\theta_{2} .
$$


Assume a function $F(\theta)$, where $\theta$ is a Grassmann variable. In light of Eq. (B.1), a Taylor expansion in terms of $\theta$ will terminate and can in general be written as

$$
F(\theta)=a+b \theta
$$

where $a$ and $b$ are ordinary complex numbers. Integrals of Grassmann variables are sometimes known as Berezin integrals and are defined such that

$$
\int d \theta F(\theta)=b
$$

In a similar fashion to derivatives, integrals do not commute. For example,

$$
\int d \theta_{1} \int d \theta_{2} \theta_{1} \theta_{2}=-\int d \theta_{1} \theta_{1} \int d \theta_{2} \theta_{2}=-\int d \theta_{1}=-1
$$

Very often, one is interested in doing integrals over the components of a complex Grassmann Weyl spinor $\theta=\left(\theta_{1}, \theta_{2}\right)$. Refer to its harmonic conjugate and its components as $\bar{\theta}=\left(\bar{\theta}_{\dot{1}}, \bar{\theta}_{\dot{2}}\right)$. Derivatives with respect to the components of $\theta$ can be written as

$$
\partial_{\alpha}=\frac{\partial}{\partial \theta^{\alpha}}, \quad \bar{\partial}^{\dot{\alpha}}=\frac{\partial}{\partial \bar{\theta}_{\dot{\alpha}}}
$$

Some other useful short notations are

$$
d^{2} \theta=d \theta_{1} d \theta_{2}, \quad d^{2} \bar{\theta}=-d \bar{\theta}_{\dot{1}} d \bar{\theta}_{\dot{2}}
$$

This leads to the convenient normalization

$$
\frac{1}{2} \int d^{2} \theta \quad \theta_{\alpha}\left(-i \sigma^{2}\right)^{\alpha \beta} \theta_{\beta}=1, \quad \frac{1}{2} \int d^{2} \bar{\theta} \bar{\theta}_{\dot{\alpha}}\left(i \sigma^{2}\right)^{\dot{\alpha} \dot{\beta}} \theta_{\dot{\beta}}=1
$$


and

$$
\frac{1}{4} \int d^{2} \theta d^{2} \bar{\theta} \quad \theta_{\alpha}\left(-i \sigma^{2}\right)^{\alpha \beta} \theta_{\beta} \bar{\theta}_{\dot{\alpha}}\left(i \sigma^{2}\right)^{\dot{\alpha} \dot{\beta}} \theta_{\dot{\beta}}=1
$$




\section{Appendix C}

\section{Van Der Waerden Notation}

This appendix summarizes the van der Waerden notation [174,175]. Its main benefit is that it simplifies the manipulation of Weyl spinors. Consider a general Dirac spinor $\psi$ as introduced in Appendix A,

$$
\psi=\left(\begin{array}{c}
\eta_{R} \\
\chi_{L}
\end{array}\right),
$$

where $\eta_{R}$ and $\chi_{L}$ are right-handed and left-handed Weyl spinors respectively. To see how it transforms under the Lorentz group, parametrize an infinitesimal rotation by $\vec{\epsilon}$. This means a right-handed rotation around the direction of the three-vector by an angle of $\epsilon$. Similarly, an infinitesimal boost can be parametrized by $\vec{\beta}$, i.e. an infinitesimal boost of $\beta$ in the direction of this vector. Using the results of Appendix A, it is easy to determine that the Weyl spinors transform as

$$
\begin{aligned}
\eta_{R} & \rightarrow\left(1+\frac{i}{2} \vec{\epsilon} \cdot \vec{\sigma}-\frac{1}{2} \vec{\beta} \cdot \vec{\sigma}\right) \eta_{R}, \\
\chi_{L} & \rightarrow\left(1+\frac{i}{2} \vec{\epsilon} \cdot \vec{\sigma}+\frac{1}{2} \vec{\beta} \cdot \vec{\sigma}\right) \chi_{L} .
\end{aligned}
$$


A short calculation then shows that

$$
i \sigma^{2} \chi_{L}^{*}
$$

transforms like a right-handed Weyl spinor. Similarly, the quantity

$$
-i \sigma^{2} \eta_{R}^{*}
$$

transforms as a left-handed Weyl spinor. These properties mean that one can freely pass from left-handed to right-handed and vice-versa. Ultimately, all Weyl spinors can be written in terms of left-handed ones only. This motivates the van der Waerden notation.

First, right-handed Weyl spinors are now written with an overline and lefthanded spinors without ones. We drop the $L$ and $R$ indices as they are now redundant and only overburden the notation. The components of $\chi$ are written as

$$
\text { components of } \chi=\chi_{\alpha} \text {. }
$$

The components of $\bar{\eta}$ are then written as

$$
\text { components of } \bar{\eta}=\bar{\eta}^{\dot{\alpha}} \text {. }
$$

Notice the dot on the superscript of $\bar{\eta}^{\dot{\alpha}}$. These can be freely added or removed by taking the complex conjugate of a spinor. For example, we have

$$
\bar{\chi}_{\dot{\alpha}}=\left(\chi_{\alpha}\right)^{*}, \quad \eta^{\alpha}=\left(\bar{\eta}^{\dot{\alpha}}\right)^{*} .
$$


Notice also the removal/adding of the overline. Indices can be raised with the help of $i \sigma^{2}$. For example, we have

$$
\chi^{\alpha}=\left(i \sigma^{2}\right)^{\alpha \beta} \chi_{\beta}, \quad \bar{\eta}^{\dot{\alpha}}=\left(i \sigma^{2}\right)^{\dot{\alpha} \dot{\beta}} \bar{\eta}_{\dot{\beta}} .
$$

The presence or absence of dots on the superscripts of $i \sigma^{2}$ is simply chosen for the indices to match and $i \sigma^{2}$ does not have an intrinsic correct way to put superscripts on it. Similarly, indices can be lowered with $-i \sigma^{2}$. For example, we have

$$
\chi_{\alpha}=\left(-i \sigma^{2}\right)_{\alpha \beta} \chi^{\beta}, \quad \bar{\eta}_{\dot{\alpha}}=\left(-i \sigma^{2}\right)_{\dot{\alpha} \dot{\beta}} \bar{\eta}^{\dot{\beta}} .
$$

To make a right-handed Weyl spinor from a left-handed spinor $\chi_{\alpha}$, it suffices to add a dot on its subscript and raise it. This is easily seen to be coherent with Eq. (C.3). This generalizes to the reverse procedure.

A dot-product can be defined between two spinors of the same handedness via

$$
\chi \cdot \eta \equiv \chi_{\alpha}\left(-i \sigma^{2}\right)^{\alpha \beta} \eta_{\beta}=\chi^{\alpha} \eta_{\alpha}, \quad \bar{\chi} \cdot \bar{\eta} \equiv \bar{\chi}^{\dot{\alpha}}\left(i \sigma^{2}\right)_{\dot{\alpha} \dot{\beta}} \bar{\eta}^{\dot{\beta}}=\bar{\chi}_{\dot{\alpha}} \bar{\eta}^{\dot{\alpha}} .
$$

Considering the Grassmann nature of spinors, it follows that

$$
\chi \cdot \eta=\eta \cdot \chi, \quad \bar{\chi} \cdot \bar{\eta}=\bar{\eta} \cdot \bar{\chi} .
$$

Using Eq. (C.2), it is easy to verify that

$$
i \sigma^{\mu} \partial_{\mu} \bar{\eta} \quad \text { and } \quad i \bar{\sigma}^{\mu} \partial_{\mu} \chi
$$

transform as left-handed and right-handed spinors respectively. This motivates the 
notation

$$
\begin{aligned}
& \text { components of } \bar{\sigma}^{\mu}=\left(\bar{\sigma}^{\mu}\right)^{\dot{\alpha} \beta}, \\
& \text { components of } \sigma^{\mu}=\left(\sigma^{\mu}\right)_{\alpha \dot{\beta}} \text {. }
\end{aligned}
$$




\section{References}

[1] H. Beauchesne, K. Earl, and T. Gregoire, The spontaneous $\mathbb{Z}_{2}$ breaking Twin Higgs, JHEP 01 (2016) 130, [arXiv:1510.06069].

[2] H. Beauchesne, K. Earl, and T. Gregoire, LHC constraints on Mini-Split anomaly and gauge mediation and prospects for LHC 14 and a future $100 \mathrm{TeV}$ pp collider, JHEP 08 (2015) 117, [arXiv: 1503.03099].

[3] CMS Collaboration, S. Chatrchyan et al., Search for supersymmetry in $p p$ collisions at $\operatorname{sqrt}(\mathrm{s})=8 \mathrm{Tev}$ in events with two opposite sign leptons, large number of jets, b-tagged jets, and large missing transverse energy., CMS-PAS-SUS-13-016, CERN, Geneva, 2013.

[4] H. Beauchesne and T. Gregoire, Electroweak precision measurements in supersymmetric models with a $U(1)_{R}$ lepton number, JHEP 05 (2014) 051, [arXiv: 1402.5403].

[5] Z. Han and W. Skiba, Effective theory analysis of precision electroweak data, Phys.Rev. D71 (2005) 075009, [hep-ph/0412166].

[6] G. Marandella, C. Schappacher, and A. Strumia, Supersymmetry and precision data after LEP2, Nucl.Phys. B715 (2005) 173-189, [hep-ph/0502095].

[7] CMS Collaboration, S. Chatrchyan et al., Measurement of the properties of a Higgs boson in the four-lepton final state, Phys.Rev. D89 (2014) 092007, [arXiv:1312.5353].

[8] ATLAS Collaboration, G. Aad et al., Measurement of the Higgs boson mass from the $H \rightarrow \gamma \gamma$ and $H \rightarrow Z Z^{*} \rightarrow 4 \ell$ channels with the ATLAS detector using $25 \mathrm{fb}^{-1}$ of pp collision data, Phys.Rev. D90 (2014) 052004, [arXiv: 1406.3827].

[9] M. E. Peskin and D. V. Schroeder, An Introduction to quantum field theory. Westview Press, 1995.

[10] C. P. Burgess and G. D. Moore, The standard model: A primer. Cambridge University Press, 2006. 
[11] K. Fujikawa, Path Integral Measure for Gauge Invariant Fermion Theories, Phys. Rev. Lett. 42 (1979) 1195-1198.

[12] K. Fujikawa, Path Integral for Gauge Theories with Fermions, Phys. Rev. D21 (1980) 2848. [Erratum: Phys. Rev.D22,1499(1980)].

[13] S. Vandoren and P. van Nieuwenhuizen, Lectures on instantons, arXiv:0802.1862.

[14] D. J. Gross and R. Jackiw, Effect of anomalies on quasirenormalizable theories, Phys. Rev. D6 (1972) 477-493.

[15] H. Georgi and S. L. Glashow, Gauge theories without anomalies, Phys. Rev. D6 (1972) 429.

[16] Particle Data Group, K. A. Olive et al., Review of Particle Physics, Chin. Phys. C38 (2014) 090001.

[17] R. D. Peccei and H. R. Quinn, CP Conservation in the Presence of Instantons, Phys. Rev. Lett. 38 (1977) 1440-1443.

[18] R. D. Peccei and H. R. Quinn, Constraints Imposed by CP Conservation in the Presence of Instantons, Phys. Rev. D16 (1977) 1791-1797.

[19] S. R. Coleman and J. Mandula, All Possible Symmetries of the S Matrix, Phys. Rev. 159 (1967) 1251-1256.

[20] R. Haag, J. T. Lopuszanski, and M. Sohnius, All Possible Generators of Supersymmetries of the s Matrix, Nucl. Phys. B88 (1975) 257.

[21] P. Labelle, Supersymmetry Demystified. McGraw-Hill, 2010.

[22] S. P. Martin, A Supersymmetry primer, hep-ph/9709356. [Adv. Ser. Direct. High Energy Phys.18,1(1998)].

[23] J. Terning, Modern Supersymmetry. Oxford Science Publications, 2006.

[24] R. Barbier, C. Berat, M. Besancon, M. Chemtob, A. Deandrea, et al., R-parity violating supersymmetry, Phys.Rept. 420 (2005) 1-202, [hep-ph/0406039].

[25] M. Dine and W. Fischler, A Phenomenological Model of Particle Physics Based on Supersymmetry, Phys.Lett. B110 (1982) 227.

[26] C. R. Nappi and B. A. Ovrut, Supersymmetric Extension of the $S U(3) x$ SU(2) $x$ U(1) Model, Phys.Lett. B113 (1982) 175.

[27] L. Alvarez-Gaume, M. Claudson, and M. B. Wise, Low-Energy Supersymmetry, Nucl.Phys. B207 (1982) 96. 
[28] M. Dine and A. E. Nelson, Dynamical supersymmetry breaking at low-energies, Phys.Rev. D48 (1993) 1277-1287, [hep-ph/9303230].

[29] M. Dine, A. E. Nelson, and Y. Shirman, Low-energy dynamical supersymmetry breaking simplified, Phys.Rev. D51 (1995) 1362-1370, [hep-ph/9408384].

[30] M. Dine, A. E. Nelson, Y. Nir, and Y. Shirman, New tools for low-energy dynamical supersymmetry breaking, Phys.Rev. D53 (1996) 2658-2669, [hep-ph/9507378].

[31] L. Randall and R. Sundrum, Out of this world supersymmetry breaking, Nucl.Phys. B557 (1999) 79-118, [hep-th/9810155].

[32] G. F. Giudice, M. A. Luty, H. Murayama, and R. Rattazzi, Gaugino mass without singlets, JHEP 9812 (1998) 027, [hep-ph/9810442].

[33] A. H. Chamseddine, R. L. Arnowitt, and P. Nath, Locally Supersymmetric Grand Unification, Phys. Rev. Lett. 49 (1982) 970.

[34] G. Burdman, Z. Chacko, H.-S. Goh, and R. Harnik, Folded supersymmetry and the LEP paradox, JHEP 02 (2007) 009, [hep-ph/0609152].

[35] Z. Chacko, H.-S. Goh, and R. Harnik, The Twin Higgs: Natural electroweak breaking from mirror symmetry, Phys. Rev. Lett. 96 (2006) 231802, [hep-ph/0506256].

[36] R. Barbieri, T. Gregoire, and L. J. Hall, Mirror world at the large hadron collider, hep-ph/0509242.

[37] H. Cai, H.-C. Cheng, and J. Terning, A Quirky Little Higgs Model, JHEP 05 (2009) 045, [arXiv:0812.0843].

[38] G. Burdman, Z. Chacko, H.-S. Goh, R. Harnik, and C. A. Krenke, The Quirky Collider Signals of Folded Supersymmetry, Phys. Rev. D78 (2008) 075028, [arXiv:0805.4667].

[39] P. Batra and Z. Chacko, A Composite Twin Higgs Model, Phys. Rev. D79 (2009) 095012, [arXiv:0811.0394].

[40] N. Craig, S. Knapen, and P. Longhi, Neutral Naturalness from Orbifold Higgs Models, Phys. Rev. Lett. 114 (2015), no. 6 061803, [arXiv:1410.6808].

[41] N. Craig, S. Knapen, and P. Longhi, The Orbifold Higgs, JHEP 03 (2015) 106, [arXiv: 1411.7393].

[42] G. Burdman, Z. Chacko, R. Harnik, L. de Lima, and C. B. Verhaaren, Colorless Top Partners, a 125 GeV Higgs, and the Limits on Naturalness, Phys. Rev. D91 (2015), no. 5 055007, [arXiv:1411.3310]. 
[43] R. Barbieri, D. Greco, R. Rattazzi, and A. Wulzer, The Composite Twin Higgs scenario, JHEP 08 (2015) 161, [arXiv: 1501.07803].

[44] M. Low, A. Tesi, and L.-T. Wang, Twin Higgs mechanism and a composite Higgs boson, Phys. Rev. D91 (2015) 095012, [arXiv:1501.07890].

[45] I. García García, R. Lasenby, and J. March-Russell, Twin higgs wimp dark matter, Phys. Rev. D 92 (Sep, 2015) 055034.

[46] N. Craig and A. Katz, The Fraternal WIMP Miracle, arXiv:1505.07113.

[47] I. García García, R. Lasenby, and J. March-Russell, Twin higgs asymmetric dark matter, Phys. Rev. Lett. 115 (Sep, 2015) 121801.

[48] M. Farina, Asymmetric Twin Dark Matter, arXiv:1506.03520.

[49] B. Batell and M. McCullough, Neutrino Masses from Neutral Top Partners, arXiv: 1504.04016.

[50] N. Craig, A. Katz, M. Strassler, and R. Sundrum, Naturalness in the Dark at the LHC, JHEP 07 (2015) 105, [arXiv: 1501.05310].

[51] D. Curtin and C. B. Verhaaren, Discovering Uncolored Naturalness in Exotic Higgs Decays, arXiv:1506.06141.

[52] D. Curtin and P. Saraswat, Towards a No-Lose Theorem for Naturalness, arXiv: 1509.04284.

[53] D. Poland and J. Thaler, The Dark Top, JHEP 11 (2008) 083, [arXiv:0808.1290].

[54] J. Scherk and J. H. Schwarz, Spontaneous Breaking of Supersymmetry Through Dimensional Reduction, Phys. Lett. B82 (1979) 60.

[55] J. Scherk and J. H. Schwarz, How to Get Masses from Extra Dimensions, Nucl. Phys. B153 (1979) 61-88.

[56] Z. Chacko, Y. Nomura, M. Papucci, and G. Perez, Natural little hierarchy from a partially goldstone twin Higgs, JHEP 01 (2006) 126, [hep-ph/0510273].

[57] S. Chang, L. J. Hall, and N. Weiner, A Supersymmetric twin Higgs, Phys. Rev. D75 (2007) 035009, [hep-ph/0604076].

[58] P. J. Fox, A. E. Nelson, and N. Weiner, Dirac gaugino masses and supersoft supersymmetry breaking, JHEP 08 (2002) 035, [hep-ph/0206096].

[59] A. Falkowski, S. Pokorski, and M. Schmaltz, Twin SUSY, Phys. Rev. D74 (2006) 035003, [hep-ph/0604066]. 
[60] J. Galloway, M. A. Luty, Y. Tsai, and Y. Zhao, Induced Electroweak Symmetry Breaking and Supersymmetric Naturalness, Phys. Rev. D89 (2014), no. 7 075003, [arXiv:1306.6354].

[61] N. Craig and K. Howe, Doubling down on naturalness with a supersymmetric twin Higgs, JHEP 03 (2014) 140, [arXiv:1312.1341].

[62] ATLAS, CMS, G. Aad et al., Combined Measurement of the Higgs Boson Mass in pp Collisions at $\sqrt{s}=7$ and 8 TeV with the ATLAS and CMS Experiments, Phys. Rev. Lett. 114 (2015) 191803, [arXiv: 1503.07589].

[63] A. Arvanitaki, N. Craig, S. Dimopoulos, and G. Villadoro, Mini-Split, JHEP 1302 (2013) 126, [arXiv: 1210.0555].

[64] R. Rattazzi, A. Strumia, and J. D. Wells, Phenomenology of deflected anomaly mediation, Nucl.Phys. B576 (2000) 3-28, [hep-ph/9912390].

[65] E. Bagnaschi, G. F. Giudice, P. Slavich, and A. Strumia, Higgs Mass and Unnatural Supersymmetry, JHEP 1409 (2014) 092, [arXiv:1407.4081].

[66] ATLAS Collaboration, G. Aad et al., Search for chargino and neutralino production in final states with one lepton, two b-jets consistent with a Higgs boson, and missing transverse momentum with the ATLAS detector in 20.3 $\mathrm{fb}^{-1}$ of $\sqrt{s}=8 \mathrm{TeV}$ pp collisions, ATLAS-CONF-2013-093, ATLAS-COM-CONF-2013-102.

[67] ATLAS Collaboration, G. Aad et al., Search for direct production of charginos, neutralinos and sleptons in final states with two leptons and missing transverse momentum in pp collisions at $\sqrt{s}=8 \mathrm{TeV}$ with the ATLAS detector, JHEP 1405 (2014) 071, [arXiv:1403.5294].

[68] ATLAS Collaboration, G. Aad et al., Search for direct production of charginos and neutralinos in events with three leptons and missing transverse momentum in $\sqrt{s}=8 T e V$ pp collisions with the ATLAS detector, JHEP 1404 (2014) 169, [arXiv: 1402.7029].

[69] ATLAS Collaboration, G. Aad et al., Search for supersymmetry at $\sqrt{s}=8$ $\mathrm{TeV}$ in final states with jets and two same-sign leptons or three leptons with the ATLAS detector, JHEP 1406 (2014) 035, [arXiv: 1404.2500].

[70] ATLAS Collaboration, G. Aad et al., Search for strong production of supersymmetric particles in final states with missing transverse momentum and at least three $b$-jets at $\sqrt{s}=8 \mathrm{TeV}$ proton-proton collisions with the ATLAS detector, JHEP 1410 (2014) 24, [arXiv:1407.0600]. 
[71] CMS Collaboration, V. Khachatryan et al., Searches for electroweak neutralino and chargino production in channels with Higgs, $Z$, and $W$ bosons in pp collisions at 8 TeV, Phys.Rev. D90 (2014) 092007, [arXiv:1409.3168].

[72] CMS Collaboration, S. Chatrchyan et al., Search for new physics in the multijet and missing transverse momentum final state in proton-proton collisions at $\sqrt{s}=8 \mathrm{TeV}$, JHEP 1406 (2014) 055, [arXiv:1402.4770].

[73] G. Giudice and A. Masiero, A Natural Solution to the mu Problem in Supergravity Theories, Phys.Lett. B206 (1988) 480-484.

[74] N. Arkani-Hamed, A. Gupta, D. E. Kaplan, N. Weiner, and T. Zorawski, Simply Unnatural Supersymmetry, arXiv:1212.6971.

[75] A. Gupta, D. E. Kaplan, and T. Zorawski, Gaugomaly Mediation Revisited, JHEP 1311 (2013) 149, [arXiv:1212.6969].

[76] D. M. Pierce, J. A. Bagger, K. T. Matchev, and R.-j. Zhang, Precision corrections in the minimal supersymmetric standard model, Nucl. Phys. B491 (1997) 3-67, [hep-ph/9606211].

[77] S. P. Martin and M. T. Vaughn, Two loop renormalization group equations for soft supersymmetry breaking couplings, Phys. Rev. D50 (1994) 2282, [hep-ph/9311340]. [Erratum: Phys. Rev.D78,039903(2008)].

[78] T. Gherghetta, G. F. Giudice, and J. D. Wells, Phenomenological consequences of supersymmetry with anomaly induced masses, Nucl.Phys. B559 (1999) 27-47, [hep-ph/9904378].

[79] M. Picariello and A. Strumia, Next-to-leading order corrections to gauge mediated gaugino masses, Nucl.Phys. B529 (1998) 81-95, [hep-ph/9802446].

[80] J. L. Feng, T. Moroi, L. Randall, M. Strassler, and S.-f. Su, Discovering supersymmetry at the Tevatron in wino LSP scenarios, Phys.Rev.Lett. 83 (1999) 1731-1734, [hep-ph/9904250].

[81] S. Jung and J. D. Wells, Gaugino physics of split supersymmetry spectrum at the LHC and future proton colliders, Phys.Rev. D89 (2014) 075004, [arXiv: 1312.1802].

[82] M. Toharia and J. D. Wells, Gluino decays with heavier scalar superpartners, JHEP 0602 (2006) 015, [hep-ph/0503175].

[83] D. Buttazzo, G. Degrassi, P. P. Giardino, G. F. Giudice, F. Sala, et al., Investigating the near-criticality of the Higgs boson, JHEP 1312 (2013) 089, [arXiv: 1307.3536]. 
[84] L. N. Mihaila, J. Salomon, and M. Steinhauser, Renormalization constants and beta functions for the gauge couplings of the Standard Model to three-loop order, Phys.Rev. D86 (2012) 096008, [arXiv: 1208.3357].

[85] M. Holthausen, K. S. Lim, and M. Lindner, Planck scale Boundary Conditions and the Higgs Mass, JHEP 1202 (2012) 037, [arXiv:1112.2415].

[86] G. F. Giudice and A. Strumia, Probing High-Scale and Split Supersymmetry with Higgs Mass Measurements, Nucl.Phys. B858 (2012) 63-83, [arXiv:1108.6077].

[87] A. Sirlin and R. Zucchini, Dependence of the Quartic Coupling H(m) on $M(H)$ and the Possible Onset of New Physics in the Higgs Sector of the Standard Model, Nucl.Phys. B266 (1986) 389.

[88] M. Cabrera, J. Casas, and A. Delgado, Upper Bounds on Superpartner Masses from Upper Bounds on the Higgs Boson Mass, Phys.Rev.Lett. 108 (2012) 021802, [arXiv:1108.3867].

[89] A. Bednyakov, A. Pikelner, and V. Velizhanin, Anomalous dimensions of gauge fields and gauge coupling beta-functions in the Standard Model at three loops, JHEP 1301 (2013) 017, [arXiv:1210.6873].

[90] A. Bednyakov, A. Pikelner, and V. Velizhanin, Yukawa coupling beta-functions in the Standard Model at three loops, Phys.Lett. B722 (2013) 336-340, [arXiv:1212.6829].

[91] A. Bednyakov, A. Pikelner, and V. Velizhanin, Higgs self-coupling beta-function in the Standard Model at three loops, Nucl.Phys. B875 (2013) 552-565, [arXiv:1303.4364].

[92] J. Barnard, B. Farmer, T. Gherghetta, and M. White, Natural gauge mediation with a bino NLSP at the LHC, Phys.Rev.Lett. 109 (2012) 241801, [arXiv:1208.6062].

[93] J. Alwall, R. Frederix, S. Frixione, V. Hirschi, F. Maltoni, et al., The automated computation of tree-level and next-to-leading order differential cross sections, and their matching to parton shower simulations, JHEP 1407 (2014) 079, [arXiv: 1405.0301].

[94] T. Sjostrand, S. Mrenna, and P. Z. Skands, PYTHIA 6.4 Physics and Manual, JHEP 0605 (2006) 026, [hep-ph/0603175].

[95] DELPHES 3, J. de Favereau et al., DELPHES 3, A modular framework for fast simulation of a generic collider experiment, JHEP 1402 (2014) 057, [arXiv: 1307.6346]. 
[96] M. Cacciari, G. P. Salam, and G. Soyez, FastJet User Manual, Eur.Phys.J. C72 (2012) 1896, [arXiv:1111.6097].

[97] M. Papucci, K. Sakurai, A. Weiler, and L. Zeune, Fastlim: a fast LHC limit calculator, Eur.Phys.J. C74 (2014), no. 11 3163, [arXiv:1402.0492].

[98] W. Beenakker, R. Hopker, M. Spira, and P. Zerwas, Squark and gluino production at hadron colliders, Nucl.Phys. B492 (1997) 51-103, [hep-ph/9610490].

[99] A. Kulesza and L. Motyka, Threshold resummation for squark-antisquark and gluino-pair production at the LHC, Phys.Rev.Lett. 102 (2009) 111802, [arXiv:0807.2405].

[100] A. Kulesza and L. Motyka, Soft gluon resummation for the production of gluino-gluino and squark-antisquark pairs at the LHC, Phys.Rev. D80 (2009) 095004, [arXiv:0905.4749].

[101] W. Beenakker, S. Brensing, M. Kramer, A. Kulesza, E. Laenen, et al., Soft-gluon resummation for squark and gluino hadroproduction, JHEP 0912 (2009) 041, [arXiv:0909.4418].

[102] W. Beenakker, S. Brensing, M. Kramer, A. Kulesza, E. Laenen, et al., Squark and Gluino Hadroproduction, Int.J.Mod.Phys. A26 (2011) 2637-2664, [arXiv:1105.1110].

[103] W. Beenakker, R. Hopker, and M. Spira, PROSPINO: A Program for the production of supersymmetric particles in next-to-leading order $Q C D$, hep-ph/9611232.

[104] A. L. Read, Presentation of search results: The CL(s) technique, J.Phys. G28 (2002) 2693-2704.

[105] T. A. W. Martin and D. Morrissey, Electroweakino constraints from LHC data, arXiv:1409.6322.

[106] CMS Collaboration, S. Chatrchyan et al., Study of the Discovery Reach in Searches for Supersymmetry at CMS with 3000/fb, CMS-PAS-FTR-13-014.

[107] T. Cohen, T. Golling, M. Hance, A. Henrichs, K. Howe, et al., SUSY Simplified Models at 14, 33, and 100 TeV Proton Colliders, JHEP 1404 (2014) 117, [arXiv:1311.6480].

[108] J. Anderson, A. Avetisyan, R. Brock, S. Chekanov, T. Cohen, et al., Snowmass Energy Frontier Simulations, arXiv:1309.1057. 
[109] A. Avetisyan, J. M. Campbell, T. Cohen, N. Dhingra, J. Hirschauer, et al., Methods and Results for Standard Model Event Generation at $\sqrt{\mathrm{s}}=14 \mathrm{TeV}$, $33 \mathrm{TeV}$ and $100 \mathrm{TeV}$ Proton Colliders (A Snowmass Whitepaper), arXiv: 1308.1636.

[110] I. Hinchliffe and F. Paige, High mass supersymmetry with high-energy hadron colliders, hep-ph/0201141.

[111] ATLAS, G. Aad et al., Search for charginos nearly mass degenerate with the lightest neutralino based on a disappearing-track signature in pp collisions at $\sqrt{(s)}=8 T e V$ with the ATLAS detector, Phys. Rev. D88 (2013), no. 11 112006, [arXiv:1310.3675].

[112] L. Hall and L. Randall, U(1)-R symmetric supersymmetry, Nucl.Phys. B352 (1991) 289-308.

[113] L. Randall and N. Rius, The Minimal U(1)-R symmetric model revisited, Phys.Lett. B286 (1992) 299-306.

[114] A. E. Nelson, N. Rius, V. Sanz, and M. Unsal, The Minimal supersymmetric model without a mu term, JHEP 0208 (2002) 039, [hep-ph/0206102].

[115] Z. Chacko, P. J. Fox, and H. Murayama, Localized supersoft supersymmetry breaking, Nucl.Phys. B706 (2005) 53-70, [hep-ph/0406142].

[116] G. D. Kribs, E. Poppitz, and N. Weiner, Flavor in supersymmetry with an extended R-symmetry, Phys.Rev. D78 (2008) 055010, [arXiv:0712.2039].

[117] K. Benakli and M. Goodsell, Dirac Gauginos in General Gauge Mediation, Nucl.Phys. B816 (2009) 185-203, [arXiv:0811.4409].

[118] S. Choi, M. Drees, A. Freitas, and P. Zerwas, Testing the Majorana Nature of Gluinos and Neutralinos, Phys.Rev. D78 (2008) 095007, [arXiv:0808.2410].

[119] G. D. Kribs, T. Okui, and T. S. Roy, Viable Gravity-Mediated Supersymmetry Breaking, Phys.Rev. D82 (2010) 115010, [arXiv:1008.1798].

[120] S. Abel and M. Goodsell, Easy Dirac Gauginos, JHEP 1106 (2011) 064, [arXiv:1102.0014].

[121] R. Davies, J. March-Russell, and M. McCullough, A Supersymmetric One Higgs Doublet Model, JHEP 1104 (2011) 108, [arXiv:1103.1647].

[122] P. Kumar and E. Ponton, Electroweak Baryogenesis and Dark Matter with an approximate R-symmetry, JHEP 1111 (2011) 037, [arXiv:1107.1719]. 
[123] E. Bertuzzo and C. Frugiuele, Fitting Neutrino Physics with a $U(1)_{R}$ Lepton Number, JHEP 1205 (2012) 100, [arXiv: 1203.5340].

[124] H. Itoyama and N. Maru, D-term Dynamical Supersymmetry Breaking Generating Split N=2 Gaugino Masses of Mixed Majorana-Dirac Type, Int.J.Mod.Phys. A27 (2012) 1250159, [arXiv:1109.2276].

[125] H. Itoyama and N. Maru, D-term Triggered Dynamical Supersymmetry Breaking, Phys. Rev. D88 (2013) 025012, [arXiv:1301.7548].

[126] H. Itoyama and N. Maru, 126 GeV Higgs Boson Associated with D-term Triggered Dynamical Supersymmetry Breaking, arXiv:1312.4157.

[127] S. Charkraborty and S. Roy, Higgs boson mass, neutrino masses and mixing and keV dark matter in an U(1)R lepton number model, JHEP 1401 (2014) 101, [arXiv:1309.6538].

[128] C. Frugiuele, T. Gregoire, P. Kumar, and E. Ponton, ' $L=R$ ' - $U(1)_{R}$ as the Origin of Leptonic 'RPV', JHEP 1303 (2013) 156, [arXiv:1210.0541].

[129] G. D. Kribs and A. Martin, Supersoft Supersymmetry is Super-Safe, Phys.Rev. D85 (2012) 115014, [arXiv:1203.4821].

[130] C. Frugiuele and T. Gregoire, Making the Sneutrino a Higgs with a $U(1)_{R}$ Lepton Number, Phys.Rev. D85 (2012) 015016, [arXiv:1107.4634].

[131] C. Frugiuele, T. Gregoire, P. Kumar, and E. Ponton, ' $L=R$ ' $-U(1)_{R}$ Lepton Number at the LHC, JHEP 1305 (2013) 012, [arXiv:1210.5257].

[132] Z. Han, Electroweak constraints on effective theories with U(2) x (1) flavor symmetry, Phys.Rev. D73 (2006) 015005, [hep-ph/0510125].

[133] M. E. Peskin and T. Takeuchi, Estimation of oblique electroweak corrections, Phys.Rev. D46 (1992) 381-409.

[134] M. E. Peskin and T. Takeuchi, A New constraint on a strongly interacting Higgs sector, Phys.Rev.Lett. 65 (1990) 964-967.

[135] R. Barbieri, A. Pomarol, R. Rattazzi, and A. Strumia, Electroweak symmetry breaking after LEP-1 and LEP-2, Nucl.Phys. B703 (2004) 127-146, [hep-ph/0405040].

[136] F. Ledroit and G. Sajot, Indirect limits on $S U S Y R_{p}$ violating couplings $\lambda$ and $\lambda^{\prime}, G D R-S-008$ (1998).

[137] C. Wood, S. Bennett, D. Cho, B. Masterson, J. Roberts, et al., Measurement of parity nonconservation and an anapole moment in cesium, Science $\mathbf{2 7 5}$ (1997) 1759-1763. 
[138] P. Vetter, D. Meekhof, P. Majumder, S. Lamoreaux, and E. Fortson, Precise test of electroweak theory from a new measurement of parity nonconservation in atomic thallium, Phys.Rev.Lett. 74 (1995) 2658-2661.

[139] N. Edwards, S. Phipp, P. Baird, and S. Nakayama, Precise Measurement of Parity Nonconserving Optical Rotation in Atomic Thallium, Phys.Rev.Lett. 74 (1995) 2654-2657.

[140] NuTeV Collaboration, G. Zeller et al., A Precise determination of electroweak parameters in neutrino nucleon scattering, Phys.Rev.Lett. 88 (2002) 091802, [hep-ex/0110059].

[141] CHARM-II Collaboration, P. Vilain et al., Precision measurement of electroweak parameters from the scattering of muon-neutrinos on electrons, Phys.Lett. B335 (1994) 246-252.

[142] A. Blondel, P. Bockmann, H. Burkhardt, F. Dydak, A. Grant, et al., Electroweak Parameters From a High Statistics Neutrino Nucleon Scattering Experiment, Z.Phys. C45 (1990) 361-379.

[143] CHARM Collaboration, J. Allaby et al., A Precise Determination of the Electroweak Mixing Angle from Semileptonic Neutrino Scattering, Phys.Lett. B177 (1986) 446.

[144] CCFR Collaboration, E744 Collaboration, E770 Collaboration, K. S. McFarland et al., A Precision measurement of electroweak parameters in neutrino - nucleon scattering, Eur.Phys.J. C1 (1998) 509-513, [hep-ex/9701010].

[145] LEP Collaboration, ALEPH Collaboration, DELPHI Collaboration, L3 Collaboration, OPAL Collaboration, LEP Electroweak Working Group, SLD Electroweak Group, SLD Heavy Flavor Group,

J. Alcaraz et al., A Combination of preliminary electroweak measurements and constraints on the standard model, hep-ex/0312023.

[146] OPAL Collaboration, G. Abbiendi et al., Tests of the standard model and constraints on new physics from measurements of fermion pair production at 189-GeV to 209-GeV at LEP, Eur.Phys.J. C33 (2004) 173-212, [hep-ex/0309053].

[147] CDF Collaboration, D0 Collaboration, V. Abazov et al., Combination of $C D F$ and DØ results on $W$ boson mass and width, Phys.Rev. D70 (2004) 092008, [hep-ex/0311039]. 
[148] L3 Collaboration, P. Achard et al., Measurement of the cross section of $W$-boson pair production at LEP, Phys.Lett. B600 (2004) 22-40, [hep-ex/0409016].

[149] E. Bertuzzo, C. Frugiuele, T. Gregoire, and E. Ponton, Dirac gauginos, $R$ symmetry and the 125 GeV Higgs, JHEP 04 (2015) 089, [arXiv:1402.5432].

[150] Planck, P. A. R. Ade et al., Planck 2013 results. XVI. Cosmological parameters, Astron. Astrophys. 571 (2014) A16, [arXiv:1303.5076].

[151] G. Steigman, Observational tests of antimatter cosmologies, Ann. Rev. Astron. Astrophys. 14 (1976) 339-372.

[152] A. D. Sakharov, Violation of CP Invariance, c Asymmetry, and Baryon Asymmetry of the Universe, Pisma Zh. Eksp. Teor. Fiz. 5 (1967) 32-35. [Usp. Fiz. Nauk161,61(1991)].

[153] G. R. Farrar and M. E. Shaposhnikov, Baryon asymmetry of the universe in the standard electroweak theory, Phys. Rev. D50 (1994) 774, [hep-ph/9305275].

[154] A. G. Cohen, D. B. Kaplan, and A. E. Nelson, WEAK SCALE BARYOGENESIS, Phys. Lett. B245 (1990) 561-564.

[155] R. Fok, G. D. Kribs, A. Martin, and Y. Tsai, Electroweak Baryogenesis in R-symmetric Supersymmetry, Phys. Rev. D87 (2013), no. 5 055018, [arXiv: 1208.2784].

[156] C. Jarlskog, Commutator of the Quark Mass Matrices in the Standard Electroweak Model and a Measure of Maximal CP Violation, Phys. Rev. Lett. 55 (1985) 1039.

[157] G. W. Anderson and L. J. Hall, The Electroweak phase transition and baryogenesis, Phys. Rev. D45 (1992) 2685-2698.

[158] M. Dine, P. Huet, and R. L. Singleton, Jr., Baryogenesis at the electroweak scale, Nucl. Phys. B375 (1992) 625-648.

[159] P. B. Arnold and O. Espinosa, The Effective potential and first order phase transitions: Beyond leading-order, Phys. Rev. D47 (1993) 3546, [hep-ph/9212235]. [Erratum: Phys. Rev.D50,6662(1994)].

[160] A. G. Cohen, D. B. Kaplan, and A. E. Nelson, Progress in electroweak baryogenesis, Ann. Rev. Nucl. Part. Sci. 43 (1993) 27-70, [hep-ph/9302210]. 
[161] M. Quiros, Finite temperature field theory and phase transitions, in High energy physics and cosmology. Proceedings, Summer School, Trieste, Italy, June 29-July 17, 1998, pp. 187-259, 1999. hep-ph/9901312.

[162] Y. Cui, Natural Baryogenesis from Unnatural Supersymmetry, JHEP 12 (2013) 067, [arXiv:1309.2952].

[163] G. Arcadi, L. Covi, and M. Nardecchia, Out-of-equilibrium baryogenesis and superweakly interacting massive particle dark matter, Phys. Rev. D89 (2014), no. 9 095020, [arXiv:1312.5703].

[164] G. Arcadi, L. Covi, and M. Nardecchia, Gravitino Dark Matter and low-scale Baryogenesis, Phys. Rev. D92 (2015), no. 11 115006, [arXiv:1507.05584].

[165] D. V. Nanopoulos and S. Weinberg, Mechanisms for Cosmological Baryon Production, Phys. Rev. D20 (1979) 2484.

[166] D. E. Kaplan, M. A. Luty, and K. M. Zurek, Asymmetric Dark Matter, Phys. Rev. D79 (2009) 115016, [arXiv:0901.4117].

[167] Y. Cui and R. Sundrum, Baryogenesis for weakly interacting massive particles, Phys. Rev. D87 (2013), no. 11 116013, [arXiv:1212.2973].

[168] F. Rompineve, Weak Scale Baryogenesis in a Supersymmetric Scenario with $R$-parity violation, JHEP 08 (2014) 014, [arXiv: 1310.0840].

[169] E. W. Kolb and M. S. Turner, The Early Universe, Front. Phys. 69 (1990) $1-547$.

[170] R. E. Cutkosky, Singularities and discontinuities of Feynman amplitudes, J. Math. Phys. 1 (1960) 429-433.

[171] J. Edsjo and P. Gondolo, Neutralino relic density including coannihilations, Phys. Rev. D56 (1997) 1879-1894, [hep-ph/9704361].

[172] M.-C. Chen, TASI 2006 Lectures on Leptogenesis, in Proceedings of Theoretical Advanced Study Institute in Elementary Particle Physics : Exploring New Frontiers Using Colliders and Neutrinos (TASI 2006), pp. 123-176, 2007. hep-ph/0703087.

[173] I. Baldes, N. F. Bell, A. Millar, K. Petraki, and R. R. Volkas, The role of CP violating scatterings in baryogenesis - case study of the neutron portal, JCAP 1411 (2014), no. 11 041, [arXiv: 1410.0108].

[174] V. der Waerden B. L., Spinoranalyse, Nachr. Ges. Wiss. Gottingen Math.-Phys. 1929: 100109 (1929).

[175] O. Veblen, Geometry of two-component Spinors, Proc. Natl. Acad. Sci (1933). 